\title{
Numerical Simulations on the Biophysical Foundations of the Neuronal Extracellular Space
}

\author{
Dissertation \\ for the award of the degree \\ "Doctor rerum naturalium" \\ Division of Mathematics and Natural Sciences \\ of the Georg-August-Universität Göttingen
}

submitted by

Andres Agudelo-Toro

from Medellín, Colombia

Göttingen 2012 


\section{Thesis committee}

Dr. Andreas Neef

Department of Nonlinear Dynamics, Max Planck Institute for Dynamics and SelfOrganization, Göttingen

Bernstein Center for Computational Neuroscience, Göttingen

University Medicine Göttingen, Göttingen

Prof. Dr. Fred Wolf

Department of Nonlinear Dynamics, Max Planck Institute for Dynamics and SelfOrganization, Göttingen

Bernstein Center for Computational Neuroscience, Göttingen

Prof. Dr. Elisha Moses

Department of Physics of Complex Systems, Weizmann Institute of Science, Rehovot

\section{Examination committee}

Dr. Andreas Neef (Thesis supervisor and 1st referee)

Department of Nonlinear Dynamics, Max Planck Institute for Dynamics and SelfOrganization, Göttingen

Bernstein Center for Computational Neuroscience, Göttingen

University Medicine Göttingen, Göttingen

Prof. Dr. Jörg Enderlein (2nd referee)

Third Institute of Physics, Georg August University, Göttingen

Prof. Dr. Fred Wolf

Department of Nonlinear Dynamics, Max Planck Institute for Dynamics and SelfOrganization, Göttingen

Bernstein Center for Computational Neuroscience, Göttingen

Prof. Dr. Elisha Moses

Department of Physics of Complex Systems, Weizmann Institute of Science, Rehovot

Prof. Dr. Gert Lube

Institute of Numerical and Applied Mathematics, Georg August University, Göttingen

Prof. Dr. Florentin Wörgötter

Third Institute of Physics, Georg August University, Göttingen

Bernstein Center for Computational Neuroscience, Göttingen

Date of the oral examination: 28.11.2012 
The surprising result, that the simple replacement (19.1.14) stabilizes the FTCS scheme, is our first encounter with the fact that differencing PDEs is an art as much as a science.

Press et al. - Numerical Recipes in C 



\section{Acknowledgments}

First of all I would like to thank every member of my family for providing great support and for creating such an interesting, and at the same time protective environment during my youth. I thank my mother Alba, my father Rigo, and my wonderful sisters Adriana and Maria for all their love and patience during my absence. I wish I could name all the $80+$ members of my family but space makes it difficult. In particular I would like to mention my aunt Alejandra and my uncle Harold which created for me a fabulous and enriching childhood experience. Also, I could not say enough words to thank the bravest woman I know, my grandmother Emilia, who raised nine children and is still raising her grandchildren and grand-grandchildren.

My days in Göttingen would not had been the same without the keen times I spent with friends and colleagues. I thank all those who were around me during this wonderful period and specially during the laborious months before submission.

This work would not have been possible without the constant guidance of my supervisor Andreas Neef. Thanks a lot for your effort in educating me and providing the important physics concepts I needed for this thesis. I also thank my former supervisor C. M. Velez for his guidance in science and other matters. From the Max Planck Institute I thank Theo Geisel and Fred Wolf for hosting me under their scientific haven and from Weizmann Institute I thank Elisha Moses. I also would like to thank the staff in Max Planck, and in particular Regina Wunderlich, for making my work much easier without administrative worries.

Last and not least I thank Hans Scherberger and all my colleagues at the German Primate Center for hosting me after my doctoral work and for allowing me the time to revise this manuscript. Finally I thank Miriam, the most awesome result of my stay in Göttingen. 



\begin{abstract}
The electric activity of neurons creates extracellular potential fields. Recent findings show that these endogenous fields act back onto the neurons, contributing to synchronization of population activity. The influence of extracellular potential fields is also relevant for understanding therapeutic approaches such as transcranial direct current stimulation, transcranial magnetic stimulation and deep brain stimulation. The mutual interaction between fields and the neuronal membrane is not captured by today's modeling tools of neuronal electrophysiology, as those are based on isolated membranes in an infinite, isopotential extracellular space. Even the direct influence of the field is not correctly represented by the commonly used "activating function". While a reduced set of Maxwell's equations can be used to couple membrane currents to extraand intracellular potentials, this approach is rarely taken, most likely because adequate computational tools are missing. This thesis presents a computational method that implements this set of equations. The fundamentals of the method are thoroughly described starting from first principles, passing by the discretization procedure, and up to the solution algorithms. By introducing an implicit solver, numerical stability is attained even with large time-steps: this allows simulation times of tens of minutes instead of weeks, even for complex problems. The method was implemented as an open source software package which is now freely available to the neuroscience community. This tool allows simulation of cells under realistic conditions: a conductive, non-homogeneous space, sub-micron cell morphology, mixed boundary conditions and various ion channel properties and distributions. The extracellular fields are accurately represented, including secondary fields, which originate at inhomogeneities of the extracellular space and can reach several millivolts. Example applications of this method and the tool are also presented.
\end{abstract}




\section{Contents}

1 Introduction $\quad 13$

1.1 Previous approaches . . . . . . . . . . . . . . . . 15

1.2 Objective of this work . . . . . . . . . . . . . . . . . . . . . 17

1.3 Structure of this work . . . . . . . . . . . . . . 17

2 Fundamentals: the space/membrane equations 21

2.1 Introduction . . . . . . . . . . . . . . . . . . 21

2.2 Maxwell's equations . . . . . . . . . . . . . . . . . 22

2.3 The quasi-static approximation of Maxwell's equations . . . . . . . . . 24

2.3.1 Decay of free charge in biological media . . . . . . . . . . . . 24

2.3.2 Decoupling the feedback of the magnetic field . . . . . . . . . . 26

2.4 Volume conductor theory . . . . . . . . . . . . . . . 27

2.5 The space/membrane equations . . . . . . . . . . . . . . . . 29

2.5.1 Extracellular/Intracellular space . . . . . . . . . . . . . . 30

2.5.2 Membrane . . . . . . . . . . . . . . . . . 30

2.5 .3 Integral form . . . . . . . . . . . . . . . . . . . . 34

2.5.4 Differential form . . . . . . . . . . . . . . . . . . . . . 35

2.6 Reduction of the $\mathrm{S} / \mathrm{M}$ equations . . . . . . . . . . . . 35

2.6.1 The cable equation . . . . . . . . . . . . . . 36

2.6.2 The cable equation is a special case of the $\mathrm{S} / \mathrm{M}$ eq. . . . . . . . 37

2.7 Boundary conditions and evolution of the $\mathrm{S} / \mathrm{M}$ eqs. . . . . . . . . . . 39

2.7.1 Capacitance and ionic currents . . . . . . . . . . . 39

2.7.2 Boundary and initial values . . . . . . . . . . . . . 40

2.7.3 Endogenous current sources ................. . . . . . . . . . 41

2.7.4 Extracellular stimulation . . . . . . . . . . . . . . . . . . . . 42

2.8 Summary . . . . . . . . . . . . . . . . . . . . . . . . . . . . . 44

3 Solutions to the space/membrane equations 47

3.1 Introduction . . . . . . . . . . . . . . . . . . . 47

3.2 Potential for point and line sources of current . . . . . . . . . . . . 48

3.3 Infinite parallel membranes. . . . . . . . . . . . . . . . . 49

3.4 Extracellular stimulation of a single cell . . . . . . . . . . . . . . . 50

3.5 Cable solution for extracellular stimulation . . . . . . . . . . . . 52

3.6 Current injection and discharge of a spherical cell . . . . . . . . . . 54 
3.7 Summary . . . . . . . . . . . . . . . . . 56

4 Discretization and numerical strategy $\quad 57$

4.1 Introduction . . . . . . . . . . . . . . . . . 57

4.1 .1 Previous work . . . . . . . . . . . . . . 58

4.2 The finite element method . . . . . . . . . . . . . . . . . . 59

4.3 Solution strategy . . . . . . . . . . . . . . . . . . . . 60

4.3.1 Solution algorithm . . . . . . . . . . . . . . 62

4.4 Space discretization . . . . . . . . . . . . . . . . . . . 62

4.4.1 Weak formulation . . . . . . . . . . . . . 62

4.4.2 Matrix form of the problem . . . . . . . . . . . . . 64

4.4.3 Reduction of the system . . . . . . . . . . . . . 66

4.4.4 Efficient reduction of the system . . . . . . . . . . . 70

4.5 Time discretization . . . . . . . . . . . . . . . . . . 74

4.5.1 Forward Euler . . . . . . . . . . . . . . . . 74

4.5.2 Implicit Crank-Nicolson . . . . . . . . . . . . . . . . . . . 75

4.6 Cable solvers . . . . . . . . . . . . . . . . . . . . 77

4.7 Summary . . . . . . . . . . . . . . . . . . . . . . . . 78

5 Stability of the numerical method $\quad 81$

5.1 Introduction . . . . . . . . . . . . . . . . . . . 81

5.2 Rationale .......................... . . 81

5.3 Analysis with the space independent recurrence relation . . . . . . . . 83

5.4 Regular discretization of the space/membrane equations . . . . . . . 85

5.5 Space dependent recurrence relation criterion . . . . . . . . . . . . . . 89

5.6 von Neumann stability analysis . . . . . . . . . . . . . . . . 90

5.7 Discussion . . . . . . . . . . . . . . . . . . . . . . . . . . . . . 93

5.8 Summary . . . . . . . . . . . . . . . . . . . . . . . 94

6 Numerical method implementation $\quad 97$

6.1 Introduction . . . . . . . . . . . . . . . . . . . 97

6.1.1 State of the art . . . . . . . . . . . . . 97

6.1.2 Chapter structure . . . . . . . . . . . . . . . . . . 98

6.2 Software requirements . . . . . . . . . . . . . . . . . . . 98

6.3 Supporting infrastructure . . . . . . . . . . . . . . . . . . 100

6.4 Software design . . . . . . . . . . . . . . . . . . . . . . 102

6.5 Results and performance . . . . . . . . . . . . . . . 103

6.6 Summary . . . . . . . . . . . . . . . . . . . . . 104

7 Fast simulation of activity at cell membranes 105

8 Discussion and Outlook $\quad 123$

8.1 The solution method . . . . . . . . . . . . . . . 123

8.2 The software implementation . . . . . . . . . . . . . 125

8.2.1 Further improvement of the tool . . . . . . . . . . . . 126 
$\begin{array}{lr}\text { A Neuron electrophysiology parameters } & 129\end{array}$

A.1 Summary of passive parameters . . . . . . . . . . . . . . . . . . . . . 129

A.2 Summary of active parameters . . . . . . . . . . . . . . . . . . 129

A.3 Calculating the cable equation parameters . . . . . . . . . . . . 133

A.4 Calculating activating function injected current . . . . . . . . . . . . 134

A.5 Permittivity of the membrane . . . . . . . . . . . . . . 135

A.6 Typical fields inside the membrane . . . . . . . . . . . . . . . . 135

A.7 Typical current densities . . . . . . . . . . . . . . . . . . . . . . . . . . . . . . . . . . . . . 136

A.8 Electrophysiology Conventions . . . . . . . . . . . . . . . 137

B CHASTE-Membrane Guide $\quad 139$

C Applications of the S/M solution: TMS 149

C.1 Introduction . . . . . . . . . . . . . . . . . . . . . . . . . 149

C.2 The dense cloverleaf coil model . . . . . . . . . . . . . . . . 150

C.2.1 Numerical tool . . . . . . . . . . . . . . . . . . . . 150

C.2.2 Coil reconstruction . . . . . . . . . . . . . . . . 152

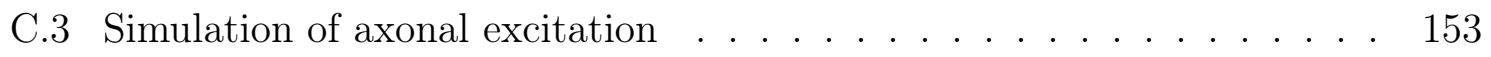

C.3.1 Passive axon . . . . . . . . . . . . . . . . . . . . . 153

C.3.2 Active axon . . . . . . . . . . . . . . . . . 154

$\begin{array}{ll}\text { D UML Design diagrams } & 157\end{array}$

E 1D Cable Utility 163

$\begin{array}{lll}\text { F Coil Simulation Tool } & 167\end{array}$

$\begin{array}{ll}\text { G Solution Algorithms } & 171\end{array}$

$\begin{array}{ll}\text { Bibliography } & 175\end{array}$

$\begin{array}{lr}\text { List of Figures } & 189\end{array}$ 


\section{Introduction}

Humans, by nature, search for simplified mind abstractions to understand complex phenomena. Minimalistic representations help visualization and the creation of tractable mathematical models. Analysis of the neuronal cell does not escape this rule. For decades, the cable equation $[3,4]$ has simplified the computational treatment of wholeneuron behavior. Large part of our quantitative understanding of neural electrophysiology is based on this equation which describes the cell as a concatenation of onedimensional entities isolated in isopotential space. Although practical, the cable equation does not fully describe the brain's electric phenomena. It has largely been known than in vivo, neurons are embedded in a dense matrix with other cells. This packing of cells is tight enough to leave only $\sim 20 \%$ of conductive space [5] and average inter-membrane distances of only tens of nanometers [6]. Work by Denk, Hayworth and others $[1,7]$ has shown the intricacies of this arrangement. Figure 1.1 presents how, a few dozen cubic micrometers of mouse brain are crossed by dozens of neuronal processes.

The electrical picture in this extracellular matrix is also excitingly rich. Neuronal activity itself, synaptic currents, sub-threshold oscillations and action currents sum-up over neuronal populations and continuously change the local extracellular potentials. Recent experimental findings in vitro and in vivo contribute to the mounting evidence that these endogenous extracellular potentials talk back to neurons and influence the synchronization of firing patterns $[8,9,10]$.

These enhanced perspectives of the neuron's environment demand for new modeling tools that can help elucidate the behavior of the cell in interaction with its surroundings. The benefits from these new tools will be countless: from understanding the influence of diffusion on synaptic signaling and ion channel conductance, to synchronization of neuronal populations, the study of the neuronal extracellular space promises to be the next frontier in neuronal modeling $[11]^{1}$.

For instance, considering of the extracellular space in neuronal models is fundamental for the development of stimulation devices. Electrical stimulation is increasingly being used for diagnostics and therapy given its benefits compared to medication. Strong, super-threshold fields are being used in the form of electroconvulsive therapy, transcranial magnetic stimulation, deep brain stimulation or closed loop cortical stimulation to activate large populations of neurons $[13,14,15,16]$. Also recently, techniques that utilize weaker, sub-threshold fields such as direct current stimulation or alternating

\footnotetext{
${ }^{1}$ Also from personal communication with NEURON's [12] developer T. Carnevale.
} 

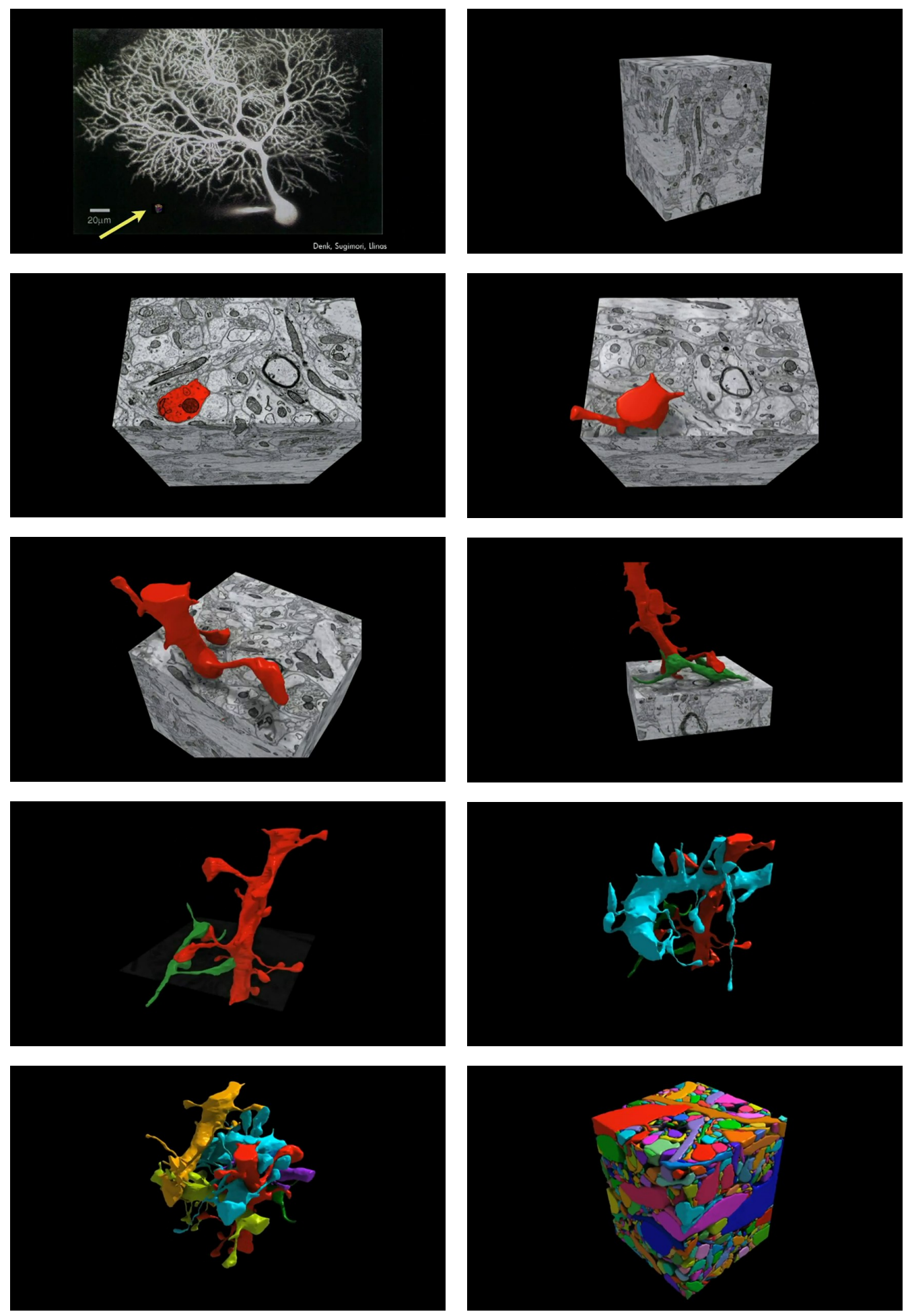

Figure 1.1: The process of Serial Block-Face Scanning Electron Microscopy [1] for reconstruction of a block of Mouse brain [2]. (Images (C) of TED Conferences LLC free for non commercial use under Creative Commons license "Attribution - NonCommercial - NonDerivative") 
current stimulation have been developed $[17,18]$ and were reported to influence motor cortex excitability, motor learning and memory [19, 20, 21] among other functions. Tools to model the brain's extracellular space will reduce experimentation costs and help develop new stimulation paradigms.

Neuroscientific applications will follow. Neuroscience research already demands for tools to calculate the extracellular potential from neuronal sources [22], and to study the unidirectional effects of extracellular potentials [23, 24, 25]. Moreover, the requirement of models of simultaneous stimulation and recording in complex set-ups has arisen over the last years for the development of multi-unit arrays and novel brain-machine interfaces [26, 27, 28, 29]. Extracellular signal recordings, such as those of the local field potential or from individual units, have been used empirically for decades in electrophysiology [30] and are critical for investigating network activity [31]. Determining the influence of the complex extracellular space in the potential signal is key to understand the shape of extracellularly recorded action potentials and in general, of local field potentials. The effect of the distribution of other cells in the potential signal is still debated [32] and new tools are required to understand better the filtering properties of extracellular media [33].

\subsection{Previous approaches}

Although a number of modeling tools have been introduced to study the interaction of extracellular potentials and neurons [34, 35, 36, 37, 38, 39] these tools are limited in their representation power. When the "forward" effect, i.e. the creation of extracellular potentials by neuronal activity is of interest, extracellular potentials are calculated with the line-source approximation [36]: the membrane currents are computed for each linear segment of a one-dimensional compartmental neuron model. These currents are then used to calculate the extracellular potential according to the "volume conductor" theory [33, 40, 41]. This approach does not consider the feed-back from extracellular potentials to the neuron and its applicability is limited to locations farther than 1 $\mathrm{km}$ away from the active membrane [36]. Interactions between adjacent cells within sub-micrometer distance, and the effect of clustering of ion channels cannot be treated. Furthermore, volume conductor theory assumes the extracellular medium to be homogeneous and isotropic. This ignores the strong secondary fields caused by the inhomogeneities of tissue [42], which give rise to the so called "virtual electrodes", and can dominate the effect of extracellular potentials on excitable tissue [43].

To simulate the changes in the cell caused by extracellular potentials i.e. the "feedback" effect, similar principles are used. The extracellular potential is computed from external current sources while assuming homogeneity and isotropy, hence ignoring the possibility of virtual electrodes. Neurons are then represented by a concatenation of one-dimensional cables and the effect of the extracellular potential is included by means of the activation function $[34,44]$. In finite cables, this effect is calculated by projecting the extracellular field on to the axis of the cables [45, 46, 47, 48].

The activating function is an approximation and has been questioned in several works $[49,50]$. Its applicability is also limited to particular geometries. A simple 

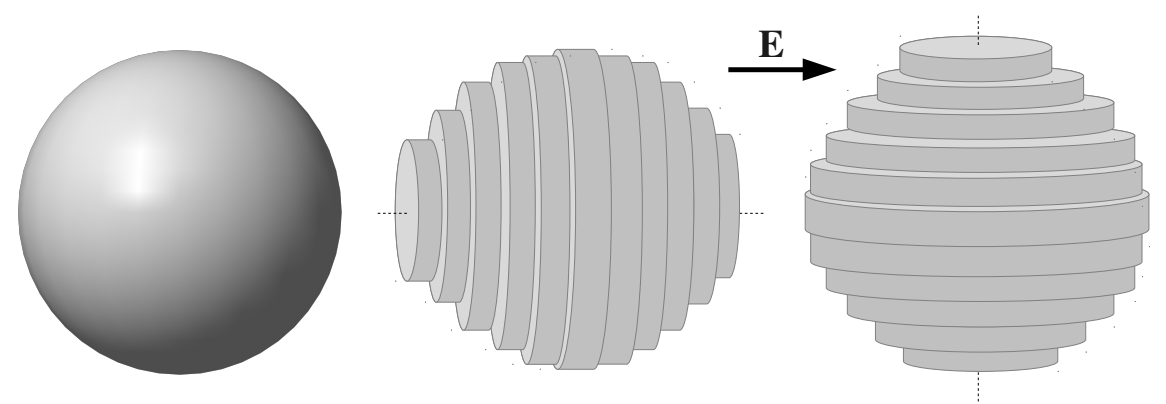

Figure 1.2: Alternative representations in the cable equation (middle, right) for a spherical cell body (left). The electric field (E) is perpendicular to the axis of the body on the right. The problems of this representation are presented in Chapter 7.

illustration of the problem is its inability to properly represent a spherical cell body inside a homogeneous field. To compute the effect, the sphere has to be approximated by cylindrical sections (see Figure 1.2). When the field is directed perpendicular to the cylinders' axes, it does not exert any influence on the cell body.

A correct solution of the forward and feed-back problem in the potential-neuron interaction requires a complete spatial representation of the neuronal membrane and its relation to the intra- and extracellular potentials. This comprises a self-consistent solution of the Laplace equation governing the potential, and the non-linear equations that determine the voltage-dependent membrane currents (i.e. the sources of the potential changes). The solution to the Laplace equation for arbitrary geometries can be achieved with finite difference (FDM), finite volume (FVM), or finite element methods (FEM). A numerical time iteration scheme then has to be selected to model the evolution of the potentials.

FDMs, FVMs and FEMs have been used to model stimulation of arbitrarily shaped cells for studies of the effects of electroporation, as well as to model simple neuronal geometries [35, 37, 51, 39, 52]. These approaches have however failed to provide a widely available tool that can fully model the neuron-extracellular potential bidirectional interaction. The deficiencies of these approaches can be summarized in three: computational limitations to represent detailed geometries, lack of efficient time evolution schemes, and the use of closed and commercial numerical tools to solve the methods. FDM approaches such as that described in [51] represent geometries in meshes with a fixed-grid spacing determined by the smallest feature in the domain. Due to the very large number of elements, the computation time of these simulations is impractically long for realistic, sub-micrometer morphological features. FEM and FVM have been employed in other cases [35, 37, 38, 39, 53, 52], but these systems have been limited to unrealistic spatial scales $[38,53]$, restrictive time evolution schemes $[35,37,38,53,52]$, two dimensions [37], and the absence of ion channels [35, 37, 38, 52]. The use of closed source $[37,38,53]$ and commercial software $[35,39,52]$ is a common feature among all these works, hindering its replicability and applicability in other scientific studies.

If the details of neuronal geometries are considered, e.g. in fine distal dendrites, or the sub-micrometer distances between cells, the importance of computationally efficient 
methods is emphasized. In the 2D simulations of Ying and Henriquez [37] for instance, the forward Euler method was used. The forward Euler method requires extremely fine time steps for micrometer geometries, limiting the physiological times that can be simulated. Besides the realistic, true sub-micron distances fundamental for the study of cell-to-cell interaction, times beyond a few milliseconds are crucial for the study of neuronal synchronization and more complex stimulation protocols.

\subsection{Objective of this work}

This thesis work is dedicated to the numerical treatment of heterogeneous, threedimensional, intra- and extracellular spaces separated by membranes with voltagedependent conductances. The main result is a computational tool to simulate these phenomena efficiently and for detailed geometries. To improve over existing tools two main strategies were used: first, the equations governing the time evolution of the electric potential in space were separated from the non-linear equations that treat the membrane currents. This follows the ideas of heart bidomain solvers [54] and a previous approach [37]. Second, an implicit time iteration scheme based on the Crank-Nicolson method [55] was introduced. This scheme allowed simulation time-steps orders of magnitude longer than the forward Euler scheme, without causing numerical instabilities. The use of this implicit method also grants applicability of adaptive time step schemes, shortening computation times by one to two orders of magnitude. It also enabled simulations to run over physiologically relevant times (tens to hundreds of microseconds), to represent the detailed, sub-micrometer cell morphology and to include ion channels with arbitrary voltage-dependent gating schemes. The new tool can represent stimulation, recording and feedback between extracellular fields and membrane voltage at realistic spatial and temporal scales. An example morphology and the resulting simulation at a fixed time step produced with the tool is presented in Figure 1.3.

\subsection{Structure of this work}

The structure of this thesis work takes the reader from general considerations on the time and space scales of electric and magnetic phenomena in biological tissue and the corresponding simplifications of Maxwell's equations (Chapter 2). Then it proceeds to the formulation of the so called "space/membrane equations" (from Section 2.5 on) and then to example solutions (Chapter 3.) The finite element formalism introduced in the method is presented in Chapter 4. Before the implementation is detailed in Chapter 6, the conditions under which the numerical method provides stable solutions are scrutinized in Chapter 5. This analysis leads to the implementation of the implicit Crank-Nicolson method in Chapter 4.5.2. The concrete software implementation of the numerical scheme is described in Chapter 6. The validation and exemplary applications of the numerical methods are summarized in a manuscript in Section 7 . This work points out the limitations of the cable equation and how the tool is superior to this method. As only free and open source software was used and the developed code was made available online, the methods described here can be directly adopted by any 
researcher with basic knowledge in the field. This aspect is particularly important, as to the author's knowledge, no comparable software is available. The software manual for installation of the tool is presented in the appendix Chapter B. An additional result of this work was the construction of a tool for the simulation of one-dimensional cables under extracellular fields and a tool for the simulation of transcranial magnetic stimulation coils. The cable tool was used to validate the results of the main threedimensional solver and is presented in appendix Chapter E. The coil tool was used to model realistic electric fields produced by transcranial magnetic stimulation coils. The realistic electric field modeling results are presented in appendix Chapter $\mathrm{C}$ and the coil tool in appendix Chapter F. 

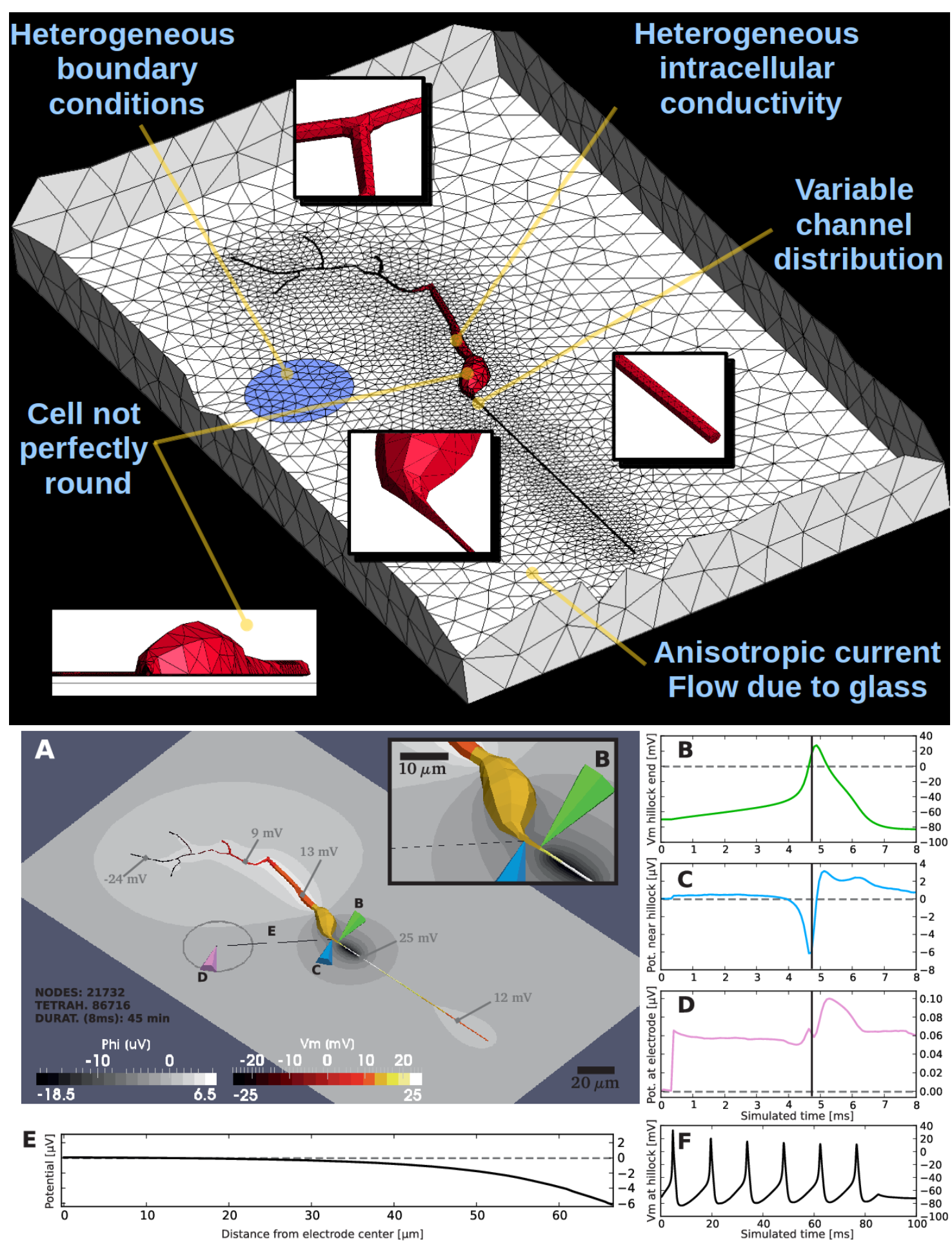

Figure 1.3: Modeling capabilities (top) and simulation results (A-F) with CHASTEMembrane. Bottom figures are described in Figure 8 of Chapter 7. 


\section{Fundamentals: the space/membrane equations}

\subsection{Introduction}

The functioning of the neuron depends on the electric field across its membrane. Neurons are filled with, and surrounded by, conductive material and the activity across their membranes produces trans-membrane currents and extracellular potentials. As physical phenomena, the neuron's activity is explained by the theory of electricity and electricity in biological matter and cells.

Several experimental and theoretical efforts have advanced directly or indirectly this theory. These began with fundamental progresses such as Thompson's studies on the propagation of signals over long cables [56], Maxwell's equations [57] and Bernstein's membrane theory [58]. Many other works followed including Hodgkin and Huxley's theory of signal propagation in the axon [3], Rall's description of current flow in the dendritic tree [4], Plonsey's volume conductor theory [59], and Nagarajan et al. stimulation of finite cables [45].

More recent works have considered the electrical phenomena around the cell. Key developments include the analysis of a single cell under external fields by Cartee and Plonsey [60], the extended cable equations of Lindsay [61], and the treatment of the volumetric membrane by Voßen and co-workers [38].

In this chapter a theory for the treatment of a three-dimensional neuron and its intraand extracellular space is presented. The equations that provide this description will be deduced from Maxwell's equations, and in particular, Maxwell's macroscopic equations [62]. These equations will be presented and will be the pillar for the description of the electrical activity of the neuron and extracellular space. A combination of Maxwell's macroscopic equations and the material properties of biological matter will lead to a more specific set of Maxwell's equations known as the quasi-static approximation.

Auxiliary equations which will help further validation of the model and its numerical solution will be deduced subsequently. Once the quasi-static reduction is obtained, the evolution of currents in conductive material will be considered in the context of the "volume conductor theory" [59]. With these two tools, the equations to represent the cell will emerge in their integral form. An important proof is then done to the equations, showing they can reduce to the well know neuronal cable equation. The way external conditions, such as boundary conditions and stimulation sources are treated is then provided. Finally a section is dedicated to consider how the magnetic field can enter 
the picture for relevant applications in neuronal stimulation.

A complete derivation of the equations describing the electric field in and around the cell, departing from basic principles up to the inclusion of the magnetic field has not been found in the previous literature. It is hopped this summary will be a useful reference for future works.

\subsection{Maxwell's equations}

Maxwell's equations describe the origin of the electric and magnetic field from charges and currents. Maxwell's general microscopic equations (also known as Maxwell's equations in empty space) allow representing the electric and magnetic vector fields from the total charge and current in a volume of space. The microscopic equations consider all charges and movement of charges, even in the atomic level where charges are bounded in atomic and molecular configurations. Maxwell's microscopic equations are:

$$
\begin{aligned}
\nabla \cdot \mathbf{E} & =\frac{\rho_{t}}{\varepsilon_{0}} \\
\nabla \cdot \mathbf{B} & =0 \\
\nabla \times \mathbf{E} & =-\frac{\partial \mathbf{B}}{\partial t}, \\
\nabla \times \mathbf{B} & =\mu_{0} \mathbf{J}_{t}+\varepsilon_{0} \mu_{0} \frac{\partial \mathbf{E}}{\partial t} .
\end{aligned}
$$

Equation (2.1) is known as Gauss's law for the electric field and charges and describes how the total charge density $\rho_{t}$ gives origin to the electric field $\mathbf{E}$. A proportionality constant known as the free space permittivity $\varepsilon_{0}$ is used to relate the quantities. Equation (2.2) is known as Gauss's law for magnetism with B the magnetic field (or more precisely the magnetic induction field) and states that the magnetic field is selenoidal and no magnetic monopoles exist. Equation (2.3) is known as Faraday's law and describes how a varying magnetic field induces an electric field. Finally, equation (2.4) is Maxwell's extended Ampere's law which describes the origin of the magnetic field by currents and by a changing electric field. Here, $\mathbf{J}_{t}$ is the total current density field (free and bound current) and the values are related by the free space permeability constant $\mu_{0}$. The term $\partial \mathbf{E} / \partial t$ on Ampere's law is the displacement current and is the correction done by Maxwell to explain how current can flow through a capacitor. Maxwell's microscopic equations are described in more detail elsewhere [63, 64].

Maxwell's macroscopic equations are

$$
\begin{aligned}
\nabla \cdot \mathbf{D} & =\rho \\
\nabla \cdot \mathbf{B} & =0 \\
\nabla \times \mathbf{E} & =-\frac{\partial \mathbf{B}}{\partial t} \\
\nabla \times \mathbf{H} & =\mathbf{J}+\frac{\partial \mathbf{D}}{\partial t} .
\end{aligned}
$$


In contrast to Maxwell's microscopic equations, Maxwell's macroscopic equations describe electrodynamics in matter and deal with free charges and currents. Eqs. (2.52.8) separate free and bounded charges and currents, explicitly representing the electric and magnetic fields bounded to the medium ${ }^{1}$. These equations factor out the bounded charges and material magnetization through special auxiliary fields: in the macroscopic equations the displacement field $\mathbf{D}$ is introduced as the sum of the electric field times $\varepsilon_{0}$ and the medium polarization $\mathbf{P}$ in the relation

$$
\mathbf{D}=\varepsilon_{0} \mathbf{E}+\mathbf{P}
$$

The magnetic field intensity $\mathbf{H}$ is also introduced and is defined as the combination of magnetic field and the medium magnetization $\mathbf{M}$,

$$
\mathbf{H}=\frac{\mathbf{B}}{\mu_{0}}-\frac{\mathbf{M}}{\mu_{0}}
$$

implying

$$
\mathbf{B}=\mu_{0} \mathbf{H}+\mathbf{M}
$$

For neuronal modeling in biological, linear materials the fields $\mathbf{P}$ and $\mathbf{M}$ can be considered parallel to $\mathbf{E}$ and $\mathbf{H}$ respectively and $\mathbf{J}$ can be considered parallel to $\mathbf{E}$ $[62,61]$. Hence constants can be added to account for them creating the expressions

$$
\begin{aligned}
& \mathbf{D}=\varepsilon_{r} \varepsilon_{0} \mathbf{E}, \\
& \mathbf{B}=\mu_{r} \mu_{0} \mathbf{H},
\end{aligned}
$$

and Ohm's law

$$
\mathbf{J}=\sigma \mathbf{E}
$$

Here $\varepsilon_{r}$ represents the relative permittivity, $\mu_{r}$ the relative permeability, and $\sigma$ the conductivity of the medium. Noting that the permittivity and permeability parameters are also usually written in a single value as $\varepsilon=\varepsilon_{r} \varepsilon_{0}$ and $\mu=\mu_{r} \mu_{0}$. Replacing in Eqs. (2.5-2.8) the Maxwell's equations for linear material take the form of Eqs. (2.10-2.13):

$$
\begin{aligned}
\nabla \cdot \varepsilon_{r} \varepsilon_{0} \mathbf{E} & =\rho, \\
\nabla \cdot \mu_{r} \mu_{0} \mathbf{H} & =0, \\
\nabla \times \mathbf{E} & =-\mu_{r} \mu_{0} \frac{\partial \mathbf{H}}{\partial t}, \\
\nabla \times \mathbf{H} & =\sigma \mathbf{E}+\varepsilon_{r} \varepsilon_{0} \frac{\partial \mathbf{E}}{\partial t} .
\end{aligned}
$$

An equation auxiliary to the Maxwell's equation is the so called continuity equation and can be now directly obtained. The continuity equation describes how current leaving a volume should be proportional to the loss in charge density inside the volume.

\footnotetext{
${ }^{1}$ Note that in (2.8) the terms $\rho$ and $\mathbf{J}$ represent the free charge density and the free current density field instead of the total charge and total current density $\rho_{t}$ and $\mathbf{J}_{t}$.
} 
Returning to Ampere-Maxwell's equation (2.8) and employing the vector identity $\nabla$. $(\nabla \times \mathbf{H})=0$ in a material with permittivity constant in time, it is true that

$$
\begin{aligned}
\nabla \cdot(\nabla \times \mathbf{H}) & =\nabla \cdot\left(\mathbf{J}+\frac{\partial \mathbf{D}}{\partial t}\right) \\
0 & =\nabla \cdot\left(\mathbf{J}+\varepsilon \frac{\partial \mathbf{E}}{\partial t}\right) .
\end{aligned}
$$

The continuity equation emerges as

$$
\nabla \cdot \mathbf{J}=-\nabla \cdot \varepsilon \frac{\partial \mathbf{E}}{\partial t}
$$

Eq. (2.14) can be also expressed in the more familiar form

$$
\nabla \cdot \mathbf{J}=-\frac{\partial \rho}{\partial t}
$$

considering that $\nabla \cdot \varepsilon \mathbf{E}=\rho$ from Eq. (2.21). Equations (2.10-2.13) and (2.14) will be base for an electric theory of the neuronal membrane.

\subsection{The quasi-static approximation of Maxwell's equations}

The set of macroscopic Maxwell's Eqs. (2.5-2.8) describe complex phenomena evolving on time scales of nanoseconds to seconds. Neuronal activity of interest can extend from milliseconds to seconds. Simulating the full set of Eqs. (2.5-2.8) in the scale of seconds of activity would be an excessively demanding computational task. However a closer inspection of the space and time scales in which these equations are of interest, and of the parameter values in living matter, shows that some their terms can be neglected.

The typical temporal dimensions where neuronal electric events develop, together with the permittivity $\varepsilon$ and conductivity $\sigma$ of the extracellular medium imply that charges decay instantly in the extracellular space. Any free, unbalanced charge is balanced in the medium within fractions of a nanosecond, being faster than the scale at which the electric processes of interest unfold. Secondly, the typical spatial and temporal dimensions, together with the permeability $\mu$ and conductivity $\sigma$ of the extracellular medium, allow to decouple the feedback of the magnetic field onto the electric field. This implies that electromagnetic wave propagation does not play an important role at the scales observed. This approximation takes the name of quasi-static Maxwell's equations $[65,61,62]$ and is introduced next.

\subsubsection{Decay of free charge in biological media}

First the decay of free charge is considered. Taking the divergence on (2.13) and using the mathematical identity $\nabla \cdot \nabla \times \mathbf{H}=0$ the following is true:

$$
0=\nabla \cdot\left(\sigma \mathbf{E}+\varepsilon_{r} \varepsilon_{0} \frac{\partial \mathbf{E}}{\partial t}\right)
$$


To estimate the relative magnitude of the summands, a spatially variable field oscillating with frequency $\omega$ is considered:

$$
\mathbf{E}(\mathbf{x}, t)=\mathbf{E}_{0}(\mathbf{x}) \exp (i \omega t)
$$

Taking the time derivative and replacing the temporal derivative in (2.15) produces

$$
\nabla \cdot\left(\sigma \mathbf{E}+i \omega \varepsilon_{r} \varepsilon_{0} \mathbf{E}\right)=0
$$

The term preceded by $i \omega \varepsilon_{r} \varepsilon_{0}$ is negligible if this ratio is much smaller than 1 :

$$
\frac{\omega \varepsilon_{r} \varepsilon_{0}}{\sigma} \ll 1
$$

Considering a typical frequency for neuronal phenomena $\omega=1000 \mathrm{~Hz}$, using the relative permittivity constant of water $\varepsilon_{r}=81$, taking a typical intracellular and extracellular conductivity of $\sigma=10 \mathrm{mS} \cdot \mathrm{cm}^{-1}=1 S \cdot \mathrm{m}^{-1}$ (see Appendix A), and the permittivity of free space in convenient units $\varepsilon_{0}=8.85 \times 10^{-12} \mathrm{~s} \cdot \mathrm{S} \cdot \mathrm{m}^{-1}$, this ratio is

$$
\frac{\omega \varepsilon_{r} \varepsilon_{0}}{\sigma}=\frac{2 \pi \times 10^{3} s^{-1} \times 81 \times 8.85 \times 10^{-12} s \cdot S \cdot m^{-1}}{1 S \cdot m^{-1}}=4.5 \times 10^{-6} .
$$

The value is much smaller than 1 indicating that the current from the temporal change of the electric field in the medium is negligible.

Lindsay et al. [61] provides an alternative reduction. Eq. (2.13) can also be written as

$$
\nabla \times \mathbf{H}=\sigma\left(\mathbf{E}+\tau_{\varepsilon} \frac{\partial \mathbf{E}}{\partial t}\right), \tau_{\varepsilon}=\frac{\varepsilon_{r} \varepsilon_{0}}{\sigma} .
$$

Taking the divergence in both sides of the equation

$$
0=\sigma\left(\nabla \cdot \mathbf{E}+\tau_{\varepsilon} \nabla \cdot \frac{\partial \mathbf{E}}{\partial t}\right)
$$

and using Gauss's law $\nabla \cdot \mathbf{E}=\frac{\rho}{\varepsilon_{r} \varepsilon_{0}}$ results in the partial differential equation

$$
\frac{\partial \rho}{\partial t}=-\frac{\rho}{\tau_{\varepsilon}}
$$

The general solution for this equation is

$$
\rho(\mathbf{x}, t)=\rho(x, 0) \exp \left(-\frac{t}{\tau_{\varepsilon}}\right)
$$

which describes the decay of free charge with initial distribution $\rho(x, 0)$. With the parameters above, $\tau_{\varepsilon} \approx 0.72 \times 10^{-9}$ seconds. The charge in the extracellular space decays in nanoseconds while events of interest are in the order of micro- and milliseconds. Also, the charge term in 2.10 is negligible. The term $\tau_{\varepsilon} \frac{\partial \mathbf{E}}{\partial t}$ is also seen to be negligible on a microsecond timescale. 
Maxwell's equations can be reduced to the set

$$
\begin{aligned}
\nabla \cdot \varepsilon_{r} \varepsilon_{0} \mathbf{E} & =0 \\
\nabla \cdot \mu_{r} \mu_{0} \mathbf{H} & =0, \\
\nabla \times \mathbf{E} & =-\mu_{r} \mu_{0} \frac{\partial \mathbf{H}}{\partial t}, \\
\nabla \times \mathbf{H} & =\sigma \mathbf{E} .
\end{aligned}
$$

The step to deduce the quasi-static form is executed next.

\subsubsection{Decoupling the feedback of the magnetic field onto the electric field}

The previous procedure justifies the instantaneous decay of free charge. The electric and magnetic field are still coupled in Eq. (2.18) but as will be seen next, part of this coupling can be disregarded. Combining (2.19) and (2.18) results in

$$
\mathbf{E}=\frac{\nabla \times \mathbf{H}}{\sigma}
$$

and

$$
\nabla \times \nabla \times \mathbf{H}=-\sigma \mu_{r} \mu_{0} \frac{\partial \mathbf{H}}{\partial t} .
$$

Vector identity $\nabla \times \nabla \times \mathbf{H}=\nabla(\nabla \cdot \mathbf{H})-\nabla \cdot(\nabla \mathbf{H})$ can be used and Gauss's law of magnetism $\nabla \cdot \mathbf{H}=0$ implies that

$$
\nabla \cdot \nabla \mathbf{H}=\sigma \mu_{r} \mu_{0} \frac{\partial \mathbf{H}}{\partial t}
$$

This equation is a Poisson's like equation and can be interpreted as diffusion of the magnetic field. For a proper comparison of the space and time differentials the equation should be non-dimensionalized. Using the non-dimensional terms $\mathbf{H}^{\prime}=\frac{\mathbf{H}}{H_{0}}, \mathbf{x}^{\prime}=\frac{x}{X_{0}}$, and $t^{\prime}=\frac{t}{T_{0}}$ results into the equation

$$
\frac{\nabla^{\prime} \cdot \nabla^{\prime} \mathbf{H}^{\prime}}{X_{0}^{2}}=\frac{\sigma \mu_{r} \mu_{0}}{T_{0}} \frac{\partial \mathbf{H}^{\prime}}{\partial t^{\prime}}
$$

As a non-ferromagnetic substance biological media has a relative permeability of $\mu_{r}=1$. A typical time and space values are $T_{0}=10^{-3} \mathrm{~s}$ and $X_{0}=10^{-5} \mathrm{~m}$, and as in the previous section $\sigma=1 S \cdot m^{-1}$ and $\mu_{0}=4 \pi \times 10^{-7}$, then

$$
\frac{\sigma \mu \mu_{0} X_{0}^{2}}{T_{0}}=\frac{1 S \cdot m^{-1} \times 1 \times 4 \pi \times 10^{-7} s \cdot S^{-1} \cdot m^{-1} \times 10^{-10} m^{2}}{10^{-3} s}=1.3 \times 10^{-13} .
$$

The impact on the electric field (proportional to the left hand side of (2.20)) of a time changing magnetic field (right side) is governed by the magnitude of this very small nondimensional parameter. This indicates that under these scales and parameters the right hand side of (2.20) is negligible. In other words the magnetic field can be determined from the electric field, but the former is not influencing the electric field back. 
The final set of Maxwell's equations is then the quasi-static approximation:

$$
\begin{aligned}
\nabla \cdot \varepsilon_{r} \varepsilon_{0} \mathbf{E} & =0, \\
\nabla \cdot \mu_{r} \mu_{0} \mathbf{H} & =0, \\
\nabla \times \mathbf{E} & =0, \\
\nabla \times \mathbf{H} & =\sigma \mathbf{E} .
\end{aligned}
$$

From Eq. (2.23) it can be concluded that the electric field is conservative and derivable from a scalar field, for convenience the negative potential field $\boldsymbol{\Phi}$ is chosen so,

$$
\mathbf{E}=-\nabla \Phi
$$

A direct consequence of Ohm's law (2.9) and Eq. (2.25) is the relation

$$
\mathbf{J}=-\sigma \nabla \boldsymbol{\Phi}
$$

And from (2.21) and (2.25) the quasi-static equations lead to Laplace's equation for the potential in biological material (such as the intra- and extracellular space):

$$
\nabla \cdot \nabla \Phi=0
$$

The intracellular and extracellular space zero divergence also applies for current densities implying

$$
\nabla \cdot \mathbf{J}=0 \text {. }
$$

This new equation gives origin to the so called volume conductor theory which describes approximately current flow in tissue. This theory is expanded next.

\subsection{Volume conductor theory}

The presence of current sources and their effect in biological conductive media is studied by the volume conductor theory [66]. Sources of current in biological media include electro-physiological processes such as action potentials, synaptic potentials and stimulating electrodes. In the previous section an approximation to represent the electric field in extra- and intracellular media was presented but the picture is not complete without field sources.

As established by the quasi-static approximation, in the intracellular and extracellular space, free charges can be disregarded. The balance of charges in this space is such, that their electric field cancels out. However measurable electric fields exist in the brain. In the quasi-static/volume conductor framework these are allowed by "endogenous" current sources. That is, although current sources can exist inside the conductive volume, the points or regions where these sources originate do not belong to the volume and are for instance boundary conditions. In Figure 2.1 the volume conductor idea is illustrated. For any small volume not touching the gray area equation, $\nabla \cdot \mathbf{J}=0$ is true. Regions at the exterior boundary of the conductor can also be the origin of currents.

\footnotetext{
${ }^{2}$ Endogenous in the meaning of "growing or developing from within or originating within".
} 


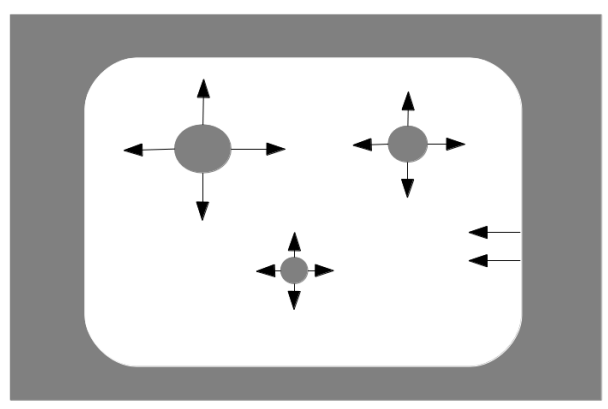

Figure 2.1: Illustration of endogenous current sources in the volume conductor. In the volume conductor (white) the quasi-static approximation applies and it is assumed that no electric field sources from free charges exist. Endogenous current sources (gray) do not belong to the volume conductor but act as boundary conditions and can introduce currents, and hence electric fields, to it.

These "endogenous" field sources are now combined with the quasi-static approximation to provide a more complete description of the intra- and extra-cellular space and they are the core of volume conductor theory.

One of the conclusions of the quasi-static equations is that given all the currents no charge sources exist as expressed by the divergence zero equation (2.28)

$$
\nabla \cdot \mathbf{J}=0 .
$$

With field J representing the free current. To introduce the endogenous sources an additional element must be added in Ohm's law (2.9) as a current density source field $\mathbf{J}_{s}$ :

$$
\mathbf{J}=\sigma \mathbf{E}+\mathbf{J}_{s} .
$$

Employing the zero divergence equation implies that

$$
\nabla \cdot\left(-\sigma \nabla \mathbf{\Phi}+\mathbf{J}_{s}\right)=0
$$

A space and time dependent volume source current can be defined as

$$
s(\mathbf{x}, t)=-\nabla \cdot \mathbf{J}_{s}
$$

and a Poisson's equation is obtained for the potential and the volume sources in

$$
-\nabla \cdot \sigma \nabla \mathbf{\Phi}=s(\mathbf{x}, t) .
$$

The way volume sources are treated, that is, determining which equation applies for the potential (Laplace's (2.27) or Poisson's (2.31)) depends on the presence or not of sources in a region of space. In any arbitrary imaginary volume where $s(\mathbf{x}, t)=0$, Laplace's equation applies. For point sources, the mathematical treatment is identical to the treatment of point charges within Gauss's law (2.1): any Gaussian surface containing a point source will have a non-zero surface flow and $-\nabla \cdot \sigma \nabla \boldsymbol{\Phi}(\mathbf{x}, t)$ will be different 


\begin{tabular}{|c|c|c|}
\hline Equation & Electrostatics & $\mathrm{QS} / \mathrm{VC}$ \\
\hline Electric field & $\mathbf{E}=-\nabla \Phi$ & $\mathbf{E}=-\nabla \boldsymbol{\Phi}$ \\
\hline Displacement field/electric current & $\mathbf{D}=\varepsilon \mathbf{E}$ & $\mathbf{J}=\sigma \mathbf{E}$ \\
\hline Electric field divergence & $\nabla \cdot \varepsilon \mathbf{E}=\rho$ & $\nabla \cdot \sigma \mathbf{E}=s$ \\
\hline Potential from point charges/currents & $\Phi=\frac{1}{4 \pi \varepsilon} \int_{V} \frac{\rho}{r} d V$ & $\boldsymbol{\Phi}=\frac{1}{4 \pi \sigma} \int_{V} \frac{s}{r} d V$ \\
\hline
\end{tabular}

Table 2.1: Electrostatics-Quasi-static/Volume conductor (QS/VC) duality.

from zero exactly in the points $\mathbf{x}$ where there are sources (e.g. in a delta function fashion). Non-punctual, volumetric sources will have a net current flow in any Gaussian surface fully containing them. Volumes such as one cell, will be in practice two volume conductors: one internal and one external, with the membrane current the boundary condition.

Considering the sources in Eq. (2.29) the equation of continuity (2.14) becomes

$$
\nabla \cdot\left(\sigma \mathbf{E}+\mathbf{J}_{s}\right)=-\nabla \cdot \varepsilon \frac{\partial \mathbf{E}}{\partial t}
$$

and in terms of volume sources (2.30) this is

$$
\nabla \cdot \sigma \mathbf{E}=-\nabla \cdot \varepsilon \frac{\partial \mathbf{E}}{\partial t}+s
$$

The quasi-static and volume conductor equations can be seen as dual to the basic electrostatic equations by replacing permittivity by conductivity and charge density by current density [47]. This duality is presented in Table 2.1.

\subsection{The space/membrane equations}

The basic tools for a description of the neuron and its extra- and intra-cellular space are now in place:

- The electric field equation (2.25),

- Ohm's law (2.26),

- Laplace's equation for the potential (2.27),

- Poisson's equation for the potential 2.31, and

- the continuity equation (2.32).

A combination of these equations will produce the intra- and extra-cellular space and membrane equations required for this work. These equations will be referred for short as the space/membrane equations.

Given the volumetric nature of the cell and the intra- and extra- cellular space, the space/membrane equations are more naturally defined from a volumetric stand point 
of imaginary enclosed Gauss's surfaces. An integral form provides a first and easy to grasp approach to the problem. After the volumetric form is obtained, a differential form of the equations will be required for the finite element formulation. This will be developed at the end of this section.

In the space/membrane equations the membrane acts as a especial boundary region where charge accumulation is allowed (capacitance) and possibly non-linear ionic flux occurs through. In the volumetric form, a piece of the membrane or a "patch" is defined easily as the divider of an imaginary volume intersecting the membrane $[61,38]$. The extracellular and intracellular space are then any volume not intersecting it (see Figure 2.2 for notation and illustration).

Charge accumulation on the membrane requires the time derivative term of Ampere's law (2.8) as preserved by the continuity equation. The staring point is then the modified continuity equation with sources $(2.32)$

$$
\nabla \cdot \sigma \mathbf{E}=-\nabla \cdot \varepsilon \frac{\partial \mathbf{E}}{\partial t}+s
$$

This equation will be valid for any volume $B$ in space. Integrating both sides for this volume results in the equation

$$
\int_{B} \nabla \cdot \sigma \mathbf{E} d V=-\int_{B} \nabla \cdot \varepsilon \frac{\partial \mathbf{E}}{\partial t} d V+\int_{B} s d V
$$

Here the term $\nabla \cdot \varepsilon \mathbf{E}$ represents free charge. With the time derivative this term indicates that the decay of charge within any volume will contribute to the total current flowing through its surface.

The treatment of Equation (2.33) will depend on whether the volume fully belongs to the intra- or extra-cellular space or intersects the membrane. These cases will be presented now.

\subsubsection{Extracellular/Intracellular space}

In the extracellular and intracellular space, equation (2.33) loses the time derivative term as specified by the quasi-static approximation. Applying Eq. (2.21), Eq. (2.33) is reduced to

$$
\int_{B} \nabla \cdot \sigma \mathbf{E} d V=\int_{B} s d V
$$

for any volume $B$ such that $B \cap \Gamma=\emptyset$. Furthermore, when no endogenous current sources are present the right hand side is zero so

$$
\int_{B} \nabla \cdot \sigma \mathbf{E} d V=0
$$

\subsubsection{Membrane}

The membrane acts as a capacitive and resistive element. Consequently, current flows through the membrane and charge can accumulate on or dissipate from the membrane. For any volume intersecting the membrane $B \cap \Gamma \neq \emptyset$ the time derivative term of the 


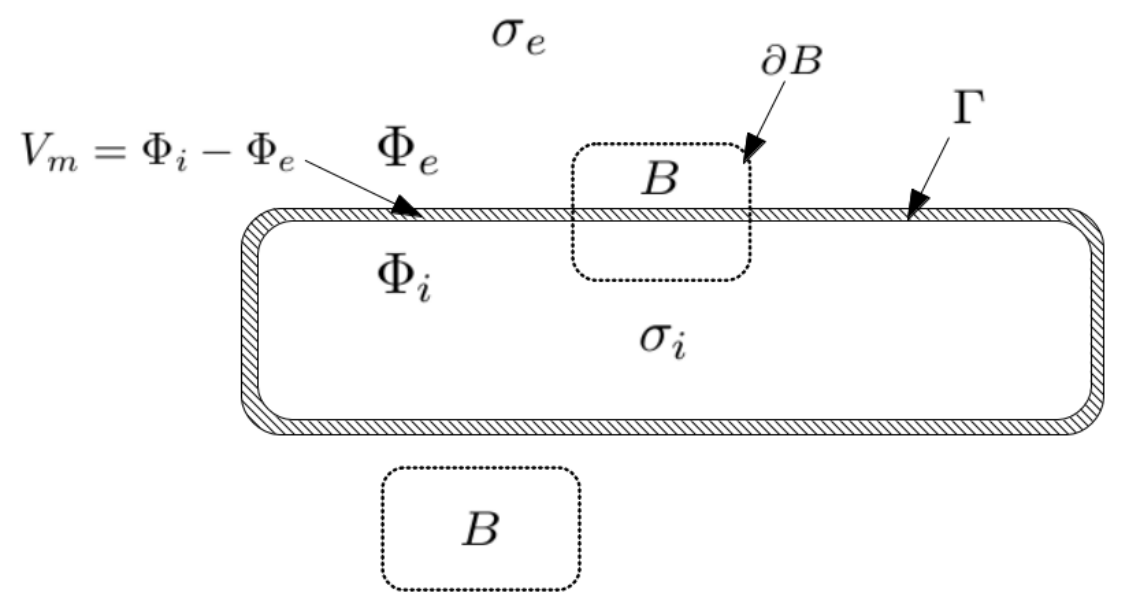

Figure 2.2: Schematic of the cell and volume regions. Volume $B$ can either intercept or not the membrane.

continuity equation remains. Applying the divergence theorem in Eq. (2.32) a more convenient surface form is obtained

$$
\int_{\partial B} \sigma \mathbf{E} \cdot \mathbf{n}_{B} d S=-\int_{\partial B} \varepsilon \frac{\partial \mathbf{E}}{\partial t} \cdot \mathbf{n}_{B} d S+\int_{B} s d V .
$$

With $\mathbf{n}_{B}$ an unitary vector normal to the surface $\partial B$ of volume $B$. Without loss of generality it will be assumed that no volume sources are found touching the membrane and that the membrane itself does not have external sources, so that the term $s$ can be dropped $^{3}$ :

$$
\int_{\partial B} \sigma \mathbf{E} \cdot \mathbf{n}_{B} d S=-\int_{\partial B} \varepsilon \frac{\partial \mathbf{E}}{\partial t} \cdot \mathbf{n}_{B} d S .
$$

This tells that there is current flowing through the membrane and charge can flow from/to it creating current. To find the influence of these fluxes on the electric field the volume intersecting the membrane will be divided in two parts: an extracellular $B_{e}$ and an intracellular $B_{i}$ part so that $B=B_{e} \cap B_{i}$ (Figure 2.3). The normal unit vector $\mathbf{n}_{B_{e}}$ will denote the normal vector in the extracellular part.

The extracellular part

$$
\int_{\partial B_{e}} \sigma_{e} \mathbf{E} \cdot \mathbf{n}_{B_{e}} d S=-\int_{\partial B_{e}} \varepsilon \frac{\partial \mathbf{E}}{\partial t} \cdot \mathbf{n}_{B_{e}} d S
$$

will now be treated. Any operation will apply for the intracellular part equivalently. In the part of the volume that touches the membrane, each side of the equality will

\footnotetext{
${ }^{3}$ For sources very close to the membrane, not touching it, a small volume can always be found that will contain the same section of membrane but that will exclude the source current.
} 


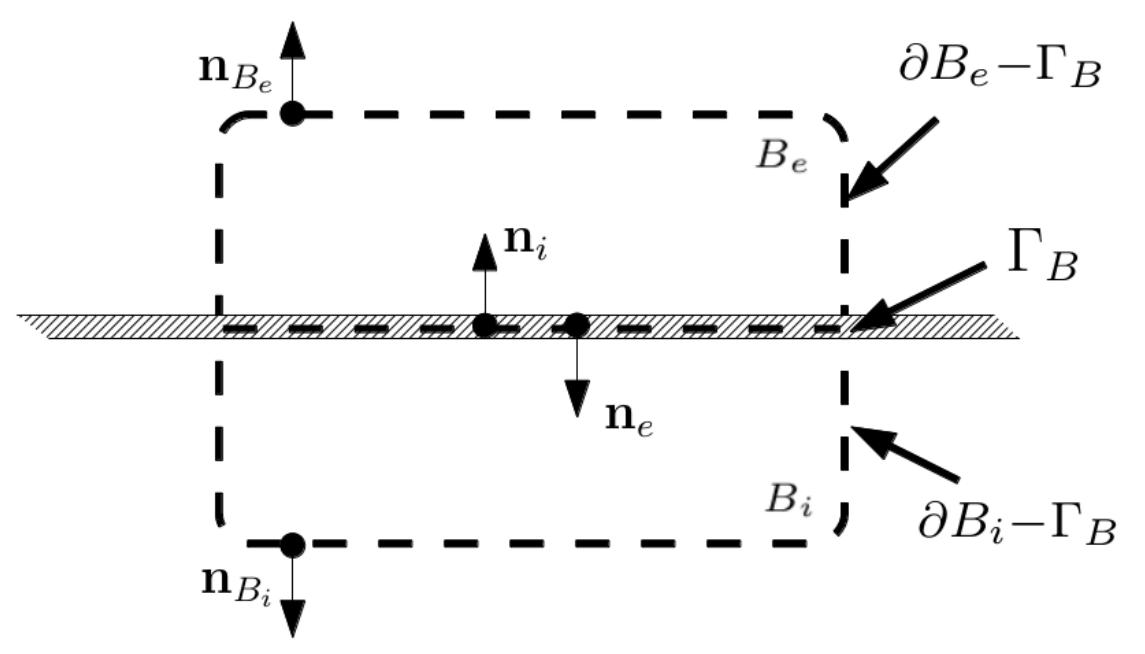

Figure 2.3: Close look to the volume intersecting the membrane. The volume is partitioned in an extracellular $B_{e}$ and an intracellular part $B_{i}$.

be associated with a different type of current flow: the left hand side term with the trans-membrane current and the right hand side with the capacitive current.

\subsubsection{Trans-membrane current}

For the left hand side term of (2.34) the surface area can be divided in two parts, one touching the membrane $\Gamma_{B}$ and the rest of the volume surface: $\partial B_{e}-\Gamma_{B}$ (Figure 2.3). For this term, the current flux in the part touching the membrane will be set to match the ionic flux $\mathbf{J}_{i o n}$. The membrane conductance here is let unspecified as different mechanisms (even non-linear) might lead to ionic current. The extracellular shell will still conserve the quasi-static properties. The new expression is

$$
\int_{\partial B_{e}} \sigma_{e} \mathbf{E} \cdot \mathbf{n}_{B_{e}} d S=\int_{\partial B_{e}-\Gamma_{B}} \sigma_{e} \mathbf{E} \cdot \mathbf{n}_{B_{e}} d S+\int_{\Gamma_{B}} \mathbf{J}_{i o n} \cdot \mathbf{n}_{e} d S
$$

The normal unitary vector of volume $B_{e}$ will be named $\mathbf{n}_{B_{e}}$ while the normal to the volume $B_{e}$ that intersects the membrane $\Gamma$ will take the notation $\mathbf{n}_{e}$.

Vector $\mathbf{n}_{e}$ preserves the orientation of the normal vector to the extracellular volume i.e. pointing towards the intracellular part. Ionic current by convention is positive out, so the scalar ionic current will be defined with a sign opposite to the extracellular volume normal

$$
I_{i o n}=-\mathbf{J}_{i o n} \cdot \mathbf{n}_{e} .
$$

Finally, the total volumetric surface current is

$$
\int_{\partial B_{e}} \sigma_{e} \mathbf{E} \cdot \mathbf{n}_{B_{e}} d S=\int_{\partial B_{e}-\Gamma_{B}} \sigma_{e} \mathbf{E} \cdot \mathbf{n}_{B_{e}} d S-\int_{\Gamma_{B}} I_{i o n} d S
$$




\subsubsection{Capacitive current}

As in the previous step the right hand side of (2.34) will be separated in the exterior shell and membrane so

$$
\int_{\partial B_{e}} \varepsilon \frac{\partial \mathbf{E}}{\partial t} \cdot \mathbf{n}_{B_{e}} d S=\int_{\partial B_{e}-\Gamma_{B}} \varepsilon \frac{\partial \mathbf{E}}{\partial t} \cdot \mathbf{n}_{B_{e}} d S+\int_{\Gamma_{B}} \varepsilon_{\Gamma} \frac{\partial \mathbf{E}}{\partial t} \cdot \mathbf{n}_{e} d S .
$$

Here $\varepsilon_{\Gamma}$ is the membrane permittivity ${ }^{4}$. From the quasi-static approximation charge accumulation in the non-membrane part $\partial B_{e}-\Gamma_{B}$ is disregarded:

$$
\int_{\partial B_{e}} \varepsilon \frac{\partial \mathbf{E}}{\partial t} \cdot \mathbf{n}_{B_{e}} d S=\int_{\Gamma_{B}} \varepsilon_{\Gamma} \frac{\partial \mathbf{E}}{\partial t} \cdot \mathbf{n}_{e} d S
$$

The membrane voltage $V_{m}$ is defined for any point on the membrane as the potential difference from the same point at on the intra- and the extracellular part:

$$
V_{m}=\Phi_{i}\left(\mathbf{x}_{i}\right)-\Phi_{e}\left(\mathbf{x}_{e}\right) \text { on } \Gamma
$$

with $\mathbf{x}_{i}, \mathbf{x}_{e}$ two points on opposite sides of the membrane and separated by membrane thickness

$$
\Delta r=\left|\mathbf{x}_{i}-\mathbf{x}_{e}\right| .
$$

As in the trans-membrane current, the capacitive current will be positive outwards. The scalar value of the electric field times the normal of the membrane surface will have opposite sign. The magnitude of the electric field on the membrane over a short $\Delta r$ can be approximated by

$$
\mathbf{E} \cdot \mathbf{n}_{e}=-\frac{V_{m}}{\Delta r}
$$

The capacitance of a flat parallel plate capacitor, such as the membrane in this case, is defined as the dielectric constant times the area, an this divided by the plates separation

$$
C=\frac{\varepsilon_{\Gamma} A_{\Gamma}}{\Delta r}
$$

The membrane capacitance will be defined by its surface area ratio $C_{m}=\frac{C}{A_{\Gamma}}$ which implies

$$
C_{m}=\frac{\varepsilon_{\Gamma}}{\Delta r}
$$

The capacitive current can then be approximated by

$$
\varepsilon_{\Gamma} \frac{\partial \mathbf{E}}{\partial t} \cdot \mathbf{n}_{e}=-\frac{\varepsilon_{\Gamma}}{\Delta r} \frac{\partial V_{m}}{\partial t}=-C_{m} \frac{\partial V_{m}}{\partial t}
$$

Finally

$$
\int_{\partial B_{e}} \varepsilon \frac{\partial \mathbf{E}}{\partial t} \cdot \mathbf{n}_{B_{e}} d S=-\int_{\Gamma_{B}} C_{m} \frac{\partial V_{m}}{\partial t} d S
$$

\footnotetext{
${ }^{4}$ See appendix Section A.5 for an estimation of this parameter.
} 


\subsubsection{Integral form}

The integral form of the space/membrane equations can now be presented. The first equation is in fact the one obtained in section 2.5.1:

$$
\int_{B} \nabla \cdot \sigma \mathbf{E} d V=\int_{B} s d V
$$

for any volume $B$.

The second equation will be the combination of membrane Eqs. (2.35) and (2.37) replaced back into $(2.34)$

$$
\begin{aligned}
\int_{\partial B_{e}} \sigma_{e} \mathbf{E} \cdot \mathbf{n}_{B_{e}} d S & =-\int_{\partial B_{e}} \varepsilon \frac{\partial \mathbf{E}}{\partial t} \cdot \mathbf{n}_{B_{e}} d S \\
\int_{\partial B_{e}-\Gamma_{B}} \sigma_{e} \mathbf{E} \cdot \mathbf{n}_{B_{e}} d S-\int_{\Gamma_{B}} I_{i o n} d S & =\int_{\Gamma_{B}} C_{m} \frac{\partial V_{m}}{\partial t} d S
\end{aligned}
$$

Reorganizing

$$
\int_{\partial B_{e}-\Gamma_{B}} \sigma_{e} \mathbf{E} \cdot \mathbf{n}_{B_{e}} d S=\int_{\Gamma_{B}}\left(C_{m} \frac{\partial V_{m}}{\partial t}+I_{i o n}\right) d S .
$$

The third equation corresponds to the intracellular part which is the same Eq. 2.39 but corrected for the oppositely oriented normal vectors

$$
\int_{\partial B_{i}-\Gamma_{B}} \sigma_{i} \mathbf{E} \cdot \mathbf{n}_{B_{i}} d S=-\int_{\Gamma_{B}}\left(C_{m} \frac{\partial V_{m}}{\partial t}+I_{i o n}\right) d S .
$$

Adding these two, corroborates that the volume $B$ intersecting the membrane has also zero net flux

$$
\int_{\partial B_{e}-\Gamma_{B}} \sigma_{e} \mathbf{E} \cdot \mathbf{n}_{B_{e}} d S+\int_{\partial B_{e}-\Gamma_{B}} \sigma_{e} \mathbf{E} \cdot \mathbf{n}_{B_{i}} d S=0
$$

implying that even for volumes intersecting the membrane the zero net current flux is preserved $^{5}$

$$
\int_{\partial B} \sigma \mathbf{E} \cdot \mathbf{n}_{B} d S=0
$$

This is equivalent to the no-source version of (2.38).

A useful definition will be to lump the capacitive and ionic currents in a single term called the membrane current

$$
I_{m}=C_{m} \frac{\partial V_{m}}{\partial t}+I_{i o n}
$$

Although the name "membrane current" has been used by others to imply the transmembrane current or the ionic currents (for instance in Dayan and Abbott's book [67]) the name "membrane current" from Hodgkin and Huxley's classical paper was adopted $[3]^{6}$. 


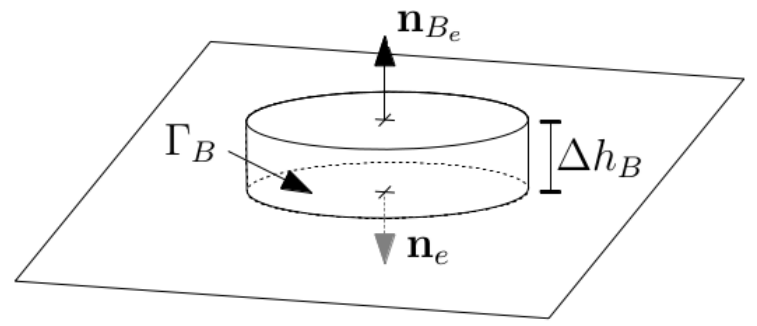

Figure 2.4: A small imaginary cylinder sitting on top of a piece of membrane.

\subsubsection{Differential form}

A differential form of the space/membrane equations, useful for the finite element definition of the problem, is now obtained. The space/membrane equations in their integral form (2.38), (2.39), (2.40) are defined for any volume B. The space Eq. (2.38) is valid for any volume and this can only be true if

$$
\nabla \cdot \sigma \mathbf{E}=s
$$

The small imaginary cylinder presented in Figure 2.4 is now required. For this small cylinder, the surface area $\partial B_{e}-\Gamma_{B}$ approximates that of $\Gamma_{B}$. For an infinitesimally short cylinder $\Delta h_{B} \rightarrow 0$ the integral areas are the same and

$$
\begin{aligned}
\int_{\Gamma_{B}} \sigma_{e} \mathbf{E} \cdot \mathbf{n}_{B_{e}} d S & =\int_{\Gamma_{B}} I_{m} d S \\
\sigma_{e} \mathbf{E} \cdot \mathbf{n}_{B_{e}} & =I_{m} .
\end{aligned}
$$

The normal $\mathbf{n}_{B_{e}}$ points to the outside of the cell but the more convenient and opposite surface normal $\mathbf{n}_{e}$ pointing inside of the cell can be used. The differential version of (2.39) and (2.40) are then

$$
I_{m}=-\sigma_{e} \mathbf{E} \cdot \mathbf{n}_{e}=\sigma_{e} \nabla \boldsymbol{\Phi}_{e} \cdot \mathbf{n}_{e}
$$

and the intracellular equivalent

$$
I_{m}=\sigma_{i} \mathbf{E} \cdot \mathbf{n}_{i}=-\sigma_{i} \nabla \boldsymbol{\Phi}_{i} \cdot \mathbf{n}_{i}
$$

\subsection{Reduction of the space/membrane equations to the cable equation}

Given the assumptions of an infinite, grounded extracellular space and a one-dimensional intracellular space, the cable equation can be deduced from the space/membrane equations. This deduction is now presented providing a validation to the system of equations. Before the derivation is presented the cable equation is first introduced.

\footnotetext{
${ }^{5}$ But not necessarily their conforming shells.

${ }^{6}$ More exactly in their paper the name "membrane current density" was used (denoted $I$ in their case). This represented the sum of capacitive and membrane current.
} 


\subsubsection{The cable equation}

The cable equation provides a mathematical model of the electric potential and the current flow in neuron fibers. This equation describes the membrane as a combination of a capacitive component representing the lipid bilayer and a purely resistive current flow representing the sub-threshold membrane leak. Conduction along the cable corresponds to a conductive ohmic current flux, dependent on the fiber's intracellular resistivity. Among others ${ }^{7}$, the two core assumptions in cable theory are: first, that the potential on any cross-section of the fiber is equal, and second, that the extracellular space is isopotential and set to be zero. The first assumption results from the relatively short fiber diameter respect to its length, so the only important flow of current occurs along it and not radially. The second assumption results from the theoretically low extracellular resistance along the cable exterior. The cable equation only considers a single dimension, namely the axis of the fiber. Although a general neuron structure can comprise fibers oriented in any direction, the cable equation can be treated then as one dimensional.

At any point along the cylindrical fiber, current can flow along the fiber or through the membrane. The intracellular current along the fiber $I_{i}$ flows through a resistive medium of resistance per unit length $r_{i}$ in $\Omega / \mathrm{cm}$ for the units used in this work. This current has an electric field associated to it namely

$$
E=-\frac{\partial V_{m}}{\partial x}
$$

With the usual convention of decaying potential indicating positive field. The intracellular or axial current equals

$$
I_{i}=\frac{1}{r_{i}} E
$$

and is positive in the fiber axis $x$ for increasing values of $x$. The two previous equations produce

$$
I_{i}=-\frac{1}{r_{i}} \frac{\partial V_{m}}{\partial x}
$$

The current can also, transversely cross the membrane so the loss of axial intracellular current corresponds to the membrane current

$$
I_{m}=-\frac{\partial I_{i}}{\partial x}
$$

The flux trough the membrane corresponds to capacitive current flow and ionic flux. Capacitance is specified for the cable equation as $c_{m}$ in units of capacitance per surface area for unit distance: $\mu \mathrm{F} / \mathrm{cm}^{2} \cdot \mathrm{cm}$.

In the case of passive flux, charges cross the membrane via an ideal linear conducting leak channel with an associated resistance $r_{m}$ for unit area per unit length $\Omega^{8} \Omega \mathrm{cm}^{2} / \mathrm{cm}$. The membrane current corresponds to

\footnotetext{
${ }^{7}$ See for instance [68] for a summary on the assumptions of the neuronal cable equation.

${ }^{8} \mathrm{~A}$ more logical unit would be conductance as it can be seen similarly to capacitance in units per area for unit distance, i.e. $S / \mathrm{cm}^{2} \cdot \mathrm{cm}$. However this is just the inverse of $\Omega \mathrm{cm}^{2} / \mathrm{cm}$.
} 


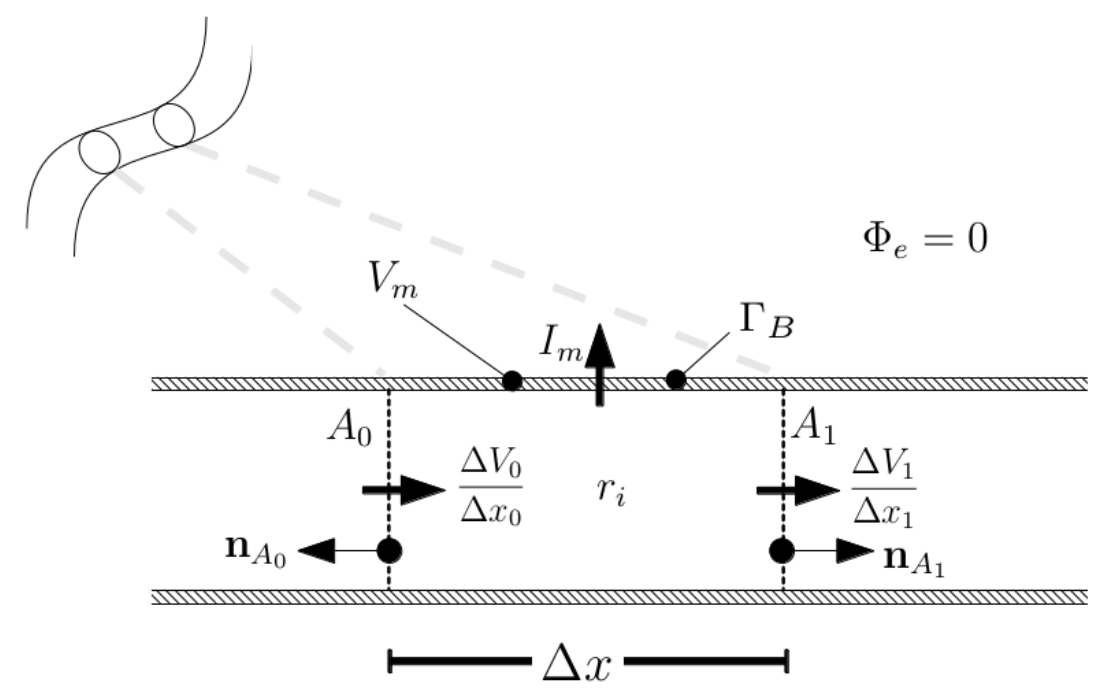

Figure 2.5: A view of the volumetric representation of the cable. One segment of length $\Delta x$ is delimited intra-cellularly by non-membrane areas $A_{0}$ and $A_{1}$.

$$
I_{m}=c_{m} \frac{\partial V_{m}}{\partial t}+\frac{V_{m}}{r_{m}}
$$

Combining 2.47 and 2.48

$$
-\frac{\partial I_{i}}{\partial x}=c_{m} \frac{\partial V_{m}}{\partial t}+\frac{V_{m}}{r_{m}}
$$

and replacing with (2.46) produces

$$
\frac{\partial}{\partial x}\left(\frac{1}{r_{i}} \frac{\partial V_{m}}{\partial x}\right)=c_{m} \frac{\partial V_{m}}{\partial t}+\frac{V_{m}}{r_{m}}
$$

Assuming a homogeneous along the cable intracellular resistance the cable equation is

$$
\frac{1}{r_{i}} \frac{\partial^{2} V_{m}}{\partial x^{2}}=c_{m} \frac{\partial V_{m}}{\partial t}+\frac{V_{m}}{r_{m}}
$$

An alternative form is defining the length and time constants [69] $\lambda=\sqrt{r_{m} / r_{i}}$ and $\tau=r_{m} c_{m}$ then

$$
\lambda^{2} \frac{\partial^{2} V_{m}}{\partial x^{2}}=\tau \frac{\partial V_{m}}{\partial t}+V_{m}
$$

\subsubsection{The cable equation is a special case of the space/membrane equations}

Beginning from the intracellular integral equation (2.40) 


$$
\int_{\partial B_{i}-\Gamma_{B}} \sigma_{i} \mathbf{E} \cdot \mathbf{n}_{B_{i}} d S=-\int_{\Gamma_{B}}\left(C_{m} \frac{\partial V_{m}}{\partial t}+I_{i o n}\right) d S
$$

define the intracellular resistivity as $R_{i}=\sigma_{i}^{-1}$, consider only passive currents $I_{i o n}=$ $V_{m} / R_{m}$ with membrane resistance for unit area $R_{m}$, and use the electric field gradient definition to obtain

$$
-\int_{A_{0}} \frac{1}{R_{i}} \nabla \boldsymbol{\Phi}_{i} \cdot \mathbf{n}_{A_{0}} d S-\int_{A_{1}} \frac{1}{R_{i}} \nabla \boldsymbol{\Phi}_{i} \cdot \mathbf{n}_{A_{1}} d S=-\int_{\Gamma_{B}}\left(C_{m} \frac{\partial V_{m}}{\partial t}+\frac{V_{m}}{R_{m}}\right) d S .
$$

The non-membrane intracellular surfaces were replaced by the cylinder caps $A_{0}$, $A_{1}$ of the intracellular cable section (see Figure 2.5). As the intracellular currents are radially homogeneous, voltages change as $\Delta V_{0}, \Delta V_{1}$ across small distances $\Delta x_{0}, \Delta x_{1}$. The electric field crossing the cylinder caps $A_{0}, A_{1}$ can be replaced the ratios of these terms. The positive direction of current is along the growing $x$ and in $\mathbf{n}_{A_{0}}$ a sign change must be applied producing:

$$
\int_{A_{0}} \frac{1}{R_{i}} \frac{\Delta V_{0}}{\Delta x_{0}} d S-\int_{A_{1}} \frac{1}{R_{i}} \frac{\Delta V_{1}}{\Delta x_{1}} d S=-\int_{\Gamma_{B}}\left(C_{m} \frac{\partial V_{m}}{\partial t}+\frac{V_{m}}{R_{m}}\right) d S .
$$

From the first assumption of the cable equation the delta terms are area independent, so the integrals are their products with area $\pi \frac{d^{2}}{4}$. With $\Delta x$ the distance of the short cable section, the cylindrical side area $\pi d \Delta x$ also replaces the right hand side integral:

$$
\pi \frac{d^{2}}{4} \frac{1}{R_{i}}\left(\frac{\Delta V_{1}}{\Delta x_{1}}-\frac{\Delta V_{0}}{\Delta x_{0}}\right)=\pi d \Delta x C_{m} \frac{\partial V_{m}}{\partial t}+\pi d \Delta x \frac{V_{m}}{R_{m}} .
$$

Factoring the $\Delta x$ term

$$
\pi \frac{d^{2}}{4} \frac{1}{R_{i}}\left(\frac{\frac{\Delta V_{1}}{\Delta x_{1}}-\frac{\Delta V_{0}}{\Delta x_{0}}}{\Delta x}\right)=\pi d C_{m} \frac{\partial V_{m}}{\partial t}+\pi d \frac{V_{m}}{R_{m}}
$$

and defining the axial resistance per unit length

$$
r_{i}=\frac{R_{i}}{\pi \frac{d^{2}}{4}}
$$

the capacitance for unit distance

$$
c_{m}=\pi d C_{m}
$$

the membrane resistance per unit length

$$
r_{m}=\frac{R_{m}}{\pi d}
$$

and taking the limit case $\Delta x_{0} \rightarrow 0, \Delta x_{1} \rightarrow 0, \Delta x \rightarrow 0$, results in the cable equation (2.50):

$$
\frac{1}{r_{i}} \frac{\partial^{2} V_{m}}{\partial^{2} x}=c_{m} \frac{\partial V_{m}}{\partial t}+\frac{V_{m}}{r_{m}}
$$




\subsection{Boundary conditions and time evolution of the space $/ \mathrm{mem}$ - brane equations}

The quasi-static assumption rules out charge accumulation or dissipation over time for the intra- and extra-cellular space. Nevertheless the space/membrane equations do not rule out potential changes over time. Change of the potentials over time in these equations is fully given by membrane charges and boundary conditions. In the membrane, charge accumulation and dissipation is allowed via capacitance while in space, the potential can be disturbed by boundary conditions such as the endogenous sources of volume conductors. The time evolution due to membrane and boundary conditions are detailed now.

\subsubsection{Capacitance and ionic currents}

Capacitance creates an effective current "through" the membrane. Charges (e.g. anions) that accumulate at the inside of the membrane lead to an accumulation of oppositely charged ions (e.g. cations) on the outside. This releases anions that precisely balance the cations trapped. The net effect is a time dependent, virtual current across the membrane. During this process charge density on the membrane is altered and the membrane voltage changes. This current is the time derivative term in Eq. (2.41):

$$
I_{m}=C_{m} \frac{\partial V_{m}}{\partial t}+I_{i o n}
$$

Ionic currents across the membrane can also be the source of changes in the potential over space. The currents depend on the membrane potential, and because the lipid bilayer is an excellent insulator, on the activity of ion channels. For specific cases the ionic current can be seen as linear and behaves similar to an ohmic leak in the membrane, in this case

$$
I_{i o n}=\frac{V_{m}}{R_{m}}
$$

This form of $I_{m}$ was already used in the cable equation. A more general case is when the current components are mediated by specific ion-channels that regulate their conductance in a voltage and time dependent manner. These can be described by nonlinear ordinary differential equations involving a state vector of gating variables and the membrane voltage as in

$$
I_{\text {ion }}=I_{\text {ion }}\left(V_{m}, \mathbf{q}_{i o n}\right)
$$

with

$$
\frac{d \mathbf{q}_{i o n}}{d t}=\mathbf{f}\left(V_{m}, \mathbf{q}_{i o n}\right)
$$

An example gating scheme is the classical Hodgkin-Huxley model [3] where the ionic 


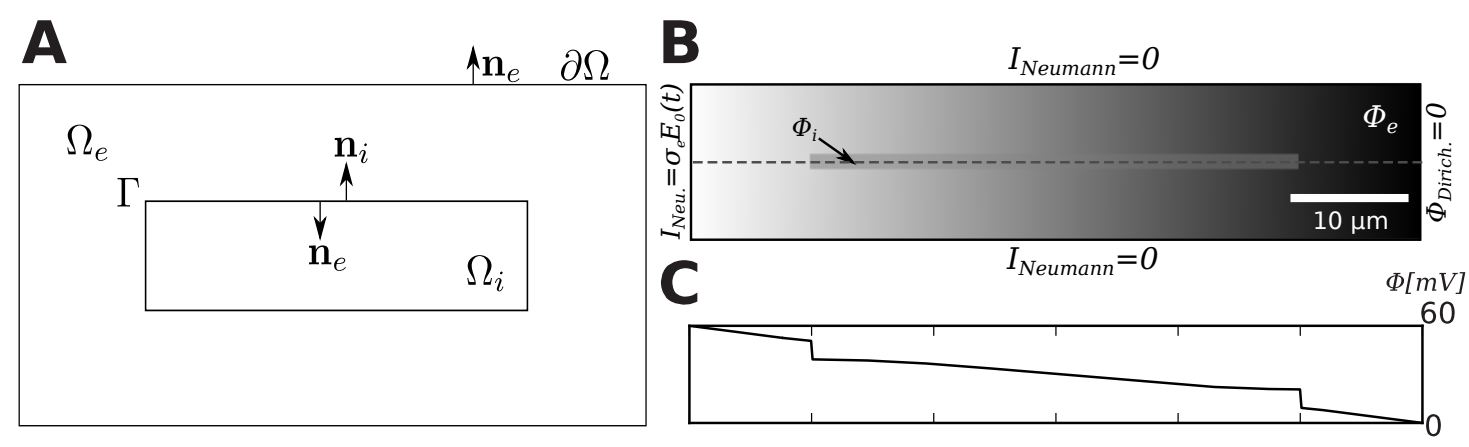

Figure 2.6: Schematic of the solution domain (SD) and example boundary conditions (BC). (A) The SD is divided in intra $\left(\Omega_{i}\right)$ and extracellular $\left(\Omega_{e}\right)$ regions, separated by a membrane $(\Gamma)$. The exterior boundary is represented by $\partial \Omega$. (B) Example of mixed boundary conditions (BC). Neumann zero flux BC are used at the top and bottom boundaries, while a Dirichlet zero BC on the right represents. The BC create extracellular $\left(\Phi_{e}\right)$ and intracellular $\left(\Phi_{i}\right)$ potential gradients. (C) Corresponding potential for the stimulus gradient along the dashed line in $(B)$.

current is described by the set of ordinary differential equations

$$
\begin{aligned}
I_{i o n} & =g_{N a} m^{3} h\left(V_{m}-E_{N a}\right)+g_{K} n^{4}\left(V_{m}-E_{K}\right)+g_{L}\left(V_{m}-E_{L}\right) \\
\frac{d m}{d t} & =(1-m) \alpha_{m}\left(V_{m}\right)-m \beta_{m}\left(V_{m}\right) \\
\frac{d h}{d t} & =(1-h) \alpha_{h}\left(V_{m}\right)-h \beta_{h}\left(V_{m}\right) \\
\frac{d n}{d t} & =(1-n) \alpha_{n}\left(V_{m}\right)-n \beta_{n}\left(V_{m}\right)
\end{aligned}
$$

Here $\mathbf{q}_{\text {ion }}=(m, h, n)^{T}$ and $E_{N a}, E_{K}$ and $E_{L}$ are the sodium, potassium and leak offset potentials [70]. The offset potentials reveal the fact that for ionic media and selective conductances the driving force of fluxes is not only electrical potential gradients but electro-chemical. Therefore current can flow across membranes even in the absence of electric potential gradients as there is a concentration gradient. Functions $\alpha_{x}$ and $\beta_{x}$ depend only on $V_{m}$ and have exponential form. More detail about these functions is beyond the scope of this work but is provided in the work of Hodgkin and Huxley [3].

\subsubsection{Boundary and initial values}

The space/membrane equations as a complete boundary and initial value problem is now defined. The problem exists in a finite domain $\Omega$ with boundaries in the exterior $\partial \Omega$ and an interior membrane $\Gamma$ (Figure 2.6(A)). The membrane boundary separates the domain in an extracellular $\Omega_{e}$ and intracellular $\Omega_{i}$ sub-domains. In the domains, material changes might exist and the conductivity coefficients can be defined as spatially dependent tensors $\sigma_{e}(x)$ and $\sigma_{i}(x)$ with $x$ the space variable. Initial conditions for the potential also might exist. In general there is a difference between the intra and extracellular potentials of the cell. The intracellular space can have an initial value 
such as

$$
\Phi_{i}=\Phi_{0}(x) \text { for } t=0 .
$$

A typical starting condition for the extracellular space will be the ground condition

$$
\Phi_{e}=0 \text { for } t=0
$$

and for the membrane current the zero flux condition

$$
I_{m}=0 \text { for } t=0
$$

On the exterior boundary $\partial \Omega_{e}$, Dirichlet, Neumann or a mixture of both boundary conditions may apply:

$$
\begin{gathered}
\Phi_{e}=\Phi_{D}(x, t) \text { on } \partial \Omega_{D} \text {, and } \\
\sigma_{e} \nabla \Phi_{e} \cdot \mathbf{n}_{e}=I_{N}(x, t) \text { on } \partial \Omega_{N} .
\end{gathered}
$$

With exclusive Dirichlet and Neumann boundaries $\partial \Omega_{D} \cap \partial \Omega_{N}=\emptyset . I_{N}$ is as a current density on the boundary and is positive when entering the domain. If at least one Dirichlet boundary is specified, the solution to the space/membrane problem is unique. When only Neumann boundary conditions are specified, the problem is solvable up to an arbitrary constant if the total current through the boundary has net flux zero [71].

Extracellular and intracellular current towards $\Gamma$ is continuous as shown for (2.43) and (2.44) so the following continuity condition applies

$$
I_{m}=\mathbf{n}_{e} \cdot \sigma_{e} \nabla \Phi_{e}=-\mathbf{n}_{i} \cdot \sigma_{i} \nabla \Phi_{i} \text { on } \Gamma
$$

Special external sources may also apply. As explained in Section 2.4 current injection anywhere behaves as a special zero dimensional boundary. Extracellular stimulation with electric and magnetically induced electric fields are also treated as boundary conditions in the space/membrane equations. This is specified next.

\subsubsection{Endogenous current sources}

In terms of potentials and the domains of Figure 2.6 the main space equation 2.42 is split in the two Poisson's equations to consider endogenous extracellular and intracellular current sources:

$$
\begin{aligned}
& -\nabla \cdot \sigma_{e}(x) \nabla \Phi_{e}(x, t)=s_{e}(x, t) \text { in } \Omega_{e} \\
& -\nabla \cdot \sigma_{i}(x) \nabla \Phi_{i}(x, t)=s_{i}(x, t) \text { in } \Omega_{i}
\end{aligned}
$$

In the absence of endogenous sources, this simplifies to Laplace's equation,

$$
-\nabla \cdot \sigma_{e} \nabla \Phi_{e}=-\nabla \cdot \sigma_{i} \nabla \Phi_{i}=0
$$




\subsubsection{Extracellular stimulation with electric and magnetic fields}

Extracellular fields such as those produced by planar electrodes or by magnetic stimulation can also be modeled with the space/membrane system. Extracellular electric stimulation can be easily represented by boundary conditions with a spatially variable Dirichlet condition in (2.51) or by flux conditions in (2.52). Grounding planar electrodes can be represented as regions in $\partial \Omega_{D}$ where the potential is zero (e.g. Figure 2.6(B)). Introducing magnetic stimulation requires a more careful analysis given the assumptions of the quasi-static approximation.

Neuronal magnetic stimulation is becoming a common technique that does not require invasive electrodes. Changing magnetic fields can induce electric fields. This induced electric field can enter the equations as a field complementary to the potential gradient. From the original Maxwell's equations, Faraday's law (2.3) relates the electric and magnetic field with

$$
\nabla \times \mathbf{E}=-\frac{\partial \mathbf{B}}{\partial t}
$$

Vector field $\mathbf{B}$ is selenoidal so a vector potential field $\mathbf{A}$ exist such that

$$
\mathbf{B}=\nabla \times \mathbf{A}
$$

Vector field $\mathbf{A}$ is known as the magnetic vector potential. In Faraday's law, replacing B one obtains

$$
\nabla \times\left(\mathbf{E}+\frac{\partial \mathbf{A}}{\partial t}\right)=0
$$

The field $\mathbf{E}+\frac{\partial \mathbf{A}}{\partial t}$ is irrotational and by vector calculus it is the gradient of a scalar field. This can even be the negative potential field ${ }^{9}$ so

$$
-\nabla \Phi=\mathbf{E}+\frac{\partial \mathbf{A}}{\partial t}
$$

Reorganizing the terms,

$$
\mathbf{E}=-\nabla \Phi-\frac{\partial \mathbf{A}}{\partial t}
$$

This expression is valid in the general Maxwell's equations framework and does not contradict the electrostatic field Equation (2.25). The electric field can be defined then as the sum of the electric potential and the time derivative of the magnetic vector potential. In the framework of the quasi-static approximation $\frac{\partial \mathbf{A}}{\partial t}$ is guaranteed to be the sole contribution of the magnetic field to the electric field (A produced by an external source). This is because secondary fields are not significant. The term $\frac{\partial \mathbf{A}}{\partial t}$ is then effectively an external electric field source not very different from the current sources in the volume conductor (e.g. Eq. (2.29)). In a conductive medium, the induced field behaves essentially as a "current everywhere" injection.

\footnotetext{
${ }^{9}$ This is better described in p. 179 of the first edition of Jackson's electrodynamics book [72]. Mathematically this can also be proven with the identity $\nabla \times(-\nabla \Phi)=0$ so $\nabla \times\left(\mathbf{E}+\frac{\partial \mathbf{A}}{\partial t}\right)=\nabla \times(-\nabla \Phi)$ and $(2.56)$ follows.
} 


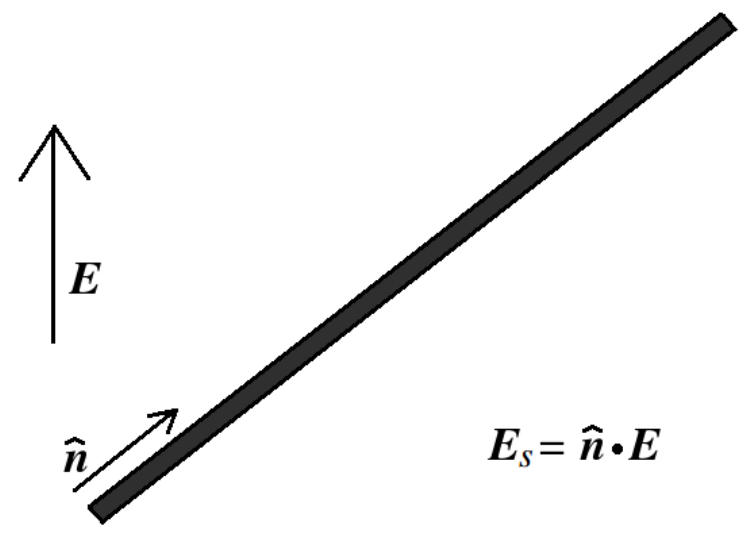

Figure 2.7: Projection of the electric field along a finite cable.

In conclusion, the magnetic field enters the space/membrane integral equations as a new source. The space integral equation (2.38) is now

$$
-\int_{B} \nabla \cdot \sigma \nabla \boldsymbol{\Phi} d V=\int_{B} s d V+\int_{B} \nabla \cdot \sigma \frac{\partial \mathbf{A}}{\partial t} d V
$$

for any volume $B^{10}$.

Assuming the magnetic field has no influence in the trans-membrane current (see appendix Section A.6 for a discussion) the magnetic field enters the intracellular and extracellular membrane equations (2.39) and (2.39) as

$$
-\int_{\partial B_{e}-\Gamma_{B}} \sigma_{e} \nabla \boldsymbol{\Phi} \cdot \mathbf{n}_{B_{e}} d S-\int_{\partial B_{e}-\Gamma_{B}} \sigma_{e} \frac{\partial \mathbf{A}}{\partial t} \cdot \mathbf{n}_{B_{e}} d S=\int_{\Gamma_{B}}\left(C_{m} \frac{\partial V_{m}}{\partial t}+I_{i o n}\right) d S
$$

and

$$
-\int_{\partial B_{i}-\Gamma_{B}} \sigma_{i} \nabla \boldsymbol{\Phi} \cdot \mathbf{n}_{B_{i}} d S-\int_{\partial B_{i}-\Gamma_{B}} \sigma_{i} \frac{\partial \mathbf{A}}{\partial t} \cdot \mathbf{n}_{B_{i}} d S=-\int_{\Gamma_{B}}\left(C_{m} \frac{\partial V_{m}}{\partial t}+I_{i o n}\right) d S
$$

In the differential pair of membrane equations this is equivalent to an additional current source term in every membrane patch scaled according to the field orientation respect to the membrane. It can be seen from the dot product that fields perpendicular to the membrane produce the largest effect.

\subsubsection{Extracellular stimulation in the cable equation}

For a proper comparison of the space/membrane equations and the cable equation in further chapters the effect of extracellular fields in the cable equation needs to be considered. Extracellular stimulation with a electric or a magnetically induced field enters the cable equation as a current source proportional to the spatial derivative of the field [44, 45]. This is shown now.

\footnotetext{
${ }^{10}$ Note that the right hand side magnetic term vanishes for homogeneous fields.
} 
The electric field expression for the cable Eq. (2.45) is modified with an additional field term $E_{s}$ which represents the external electric field component along the cable axis (Figure 2.7):

$$
E=-\frac{\partial V_{m}}{\partial x}+E_{s}
$$

Axial current $(2.46)$ is now

$$
I_{i}=\frac{1}{r_{i}}\left(-\frac{\partial V_{m}}{\partial x}+E_{s}\right)
$$

and the cable equation (2.49) takes the form

$$
\frac{\partial}{\partial x}\left(\frac{1}{r_{i}}\left(\frac{\partial V_{m}}{\partial x}-E_{s}\right)\right)=c_{m} \frac{\partial V_{m}}{\partial t}+\frac{V_{m}}{r_{m}} .
$$

Assuming a homogeneous intracellular resistance and expanding, results in

$$
\frac{1}{r_{i}} \frac{\partial^{2} V_{m}}{\partial x^{2}}-\frac{1}{r_{i}} \frac{\partial E_{s}}{\partial x}=c_{m} \frac{\partial V_{m}}{\partial t}+\frac{V_{m}}{r_{m}}
$$

Re-arranging terms, the new cable equation reads

$$
\frac{1}{r_{i}} \frac{\partial^{2} V_{m}}{\partial x^{2}}=c_{m} \frac{\partial V_{m}}{\partial t}+\frac{V_{m}}{r_{m}}+\frac{1}{r_{i}} \frac{\partial E_{s}}{\partial x}
$$

The term with the spatial derivative of the electric field is known as the activating function [34]. The external electric field component along the cable produces current flow inside the cable. If the current along the fiber changes, by continuity this current must pass through the membrane and cause stimulation. The spatial derivative along the cable describes this change. Where is the derivative different from zero? One possibility is a change in the relative orientation between cable and field as it occurs at bends of the cable. This can occur even if the field is homogeneous. This also occurs at cable ends.

Under a presence of a spatially homogeneous field, a straight cable segment of length $L$ receives a current injection described by impulse functions [73]:

$$
I_{s}(x, t)=\frac{1}{r_{i}} E_{s}(t)(\delta(x)-\delta(x-L)) .
$$

See appendix Section A.4 for an example calculation of this term in the context of cable simulators.

\subsection{Summary}

In this chapter the fundamental set of equations for resolving the activity of threedimensional cells in conductive space was introduced. The so called space/membrane equations result from the combination of the quasi-static approximation of Maxwell's equations and the Volume Conductor theory. Given the existence of the membrane boundary, the equations were first derived for convenience in their volumetric form. 
The differential form was then obtained. The integral form of the equations was used to obtain the well know cable equation demonstrating that the latter is a special case of the space/membrane equations.

The main set of equations is formed by a set of partial and ordinary differential equations. Ordinary differential equations can appear in the ionic current term. For this, proper initial and boundary conditions had to be defined for the problem. Extracellular stimulation can be represented in the model via external boundary conditions and current sources. Although current sources can be inside the solution domain, they are treated essentially as endogenous. The application of the extracellular field to a cable results in the extended cable equation with the activation function term [34]. In the following chapter a known set of solutions to the main equations will be presented. These will be useful to validate the numerical solutions in later chapters. 


\section{Solutions to the space/membrane equa- tions}

\subsection{Introduction}

This chapter provides a summary of known analytic solutions to the space/membrane equations introduced in Chapter 2. This set of solutions was used to validate the numerical schemes presented in Chapter 4, for an analysis of the stability conditions in Chapter 5, and for the numerical convergence tests presented in Chapters 5 and 7 . Solutions for the case of extracellular stimulation of a pair of infinite parallel membranes and for the finite cable are exemplified graphically and some example values are provided. These examples were really useful in understanding the behavior and properties of this set of equations.

Except for the solution of the pair of infinite parallel membranes presented in the second section, the remaining solutions in this chapter are available in the literature. Solutions to the point and line current source can be found in Plonsey's work [47]. A solution for the finite cable equation under an extracellular field was provided by Rotem et al. [73] and Monai and colleagues [74]. A more general solution to the finite cable equation for current injection at an arbitrary point can be found in Tuckwell [69] but will not be included in this summary. Solution to the extracellular stimulation of a two dimensional cell was presented by Krassowska and Neu [75]. A solution for the extracellular stimulation of a three dimensional cell was presented by Kotnik and colleagues [76]. The membrane current during current injection and the decay of potential of a charged spherical cell are also available in Plonsey's work [47]. To my knowledge, a compendium of solutions for the space/membrane has not been presented before, therefore this summary can also be a reference for future works.

A central observation in these solutions will be that the time response of the space $/ \mathrm{mem}$ brane equations evolves in two main regimes: the slow, membrane-resistance/capacitance regime and, the fast, intracellular-resistance/capacitance regime. These will be respectively associated to the well known membrane time constant $\tau_{m}$ and the less known and much shorter cell time constant $\tau_{c}$. This separation will be fundamental for the numerical treatment of the equations and reveals how, the full treatment of the electrodynamic equations for the neuron goes beyond traditional cable analysis. Although a family of time constants appears in the analytical solution of the cable equation (Section 3.5) it is usually obscured by the easier to understand membrane time constant. The membrane time constant will be seen to be dominant in the response of long cables, but for short 
cables the cell time constant primes. This difference and its relevance will become more clear in Chapters 5 and 7.

Notice that the units used in this chapter are chosen to be convenient for typical realistic currents and volumes. The units used follow the usage in the software tool (Appendix B).

\subsection{Potential for point and line sources of current}

The volume conductor introduced in Section 2.4, with two simple endogenous sources is considered. Provided no membranes exist, a solution for the potential in Eq. (2.31) in a volume $V$ with continuous charge distributions $s(\mathbf{x})$ is given by [47]

$$
\mathbf{\Phi}\left(\mathbf{x}^{\prime}\right)=\frac{1}{4 \pi \sigma} \int_{V} \frac{s(\mathbf{x})}{r} d V
$$

with $\mathbf{x}^{\prime}=\left[x^{\prime}, y^{\prime}, z^{\prime}\right]$ an arbitrary position, and the radial distance

$$
r=\sqrt{\left(x-x^{\prime}\right)^{2}+\left(y-y^{\prime}\right)^{2}+\left(z-z^{\prime}\right)^{2}} .
$$

A solution also exist for a finite number of current density point sources $s_{j}$

$$
\boldsymbol{\Phi}\left(\mathbf{x}^{\prime}\right)=\frac{1}{4 \pi \sigma} \sum_{\mathrm{j}} \frac{s_{\mathrm{j}}}{r_{\mathrm{j}}} .
$$

For a distributed linear source of current density $s(x)[\mathrm{A} / \mathrm{cm}]$ the potential differential is

$$
d \Phi=\frac{1}{4 \pi \sigma} \frac{s(x)}{r} d x
$$

$x$ the position variable along the linear source (e.g. the position along a one-dimensional neuron fiber). For any point $\mathbf{x}^{\prime}$ in space the potential is

$$
\Phi\left(\mathbf{x}^{\prime}\right)=\frac{1}{4 \pi \sigma} \int_{L} \frac{s(x)}{r} d x
$$

This is known as the line-source approximation [36]. A solution for the integral can be found for a constant current source $s_{0}$ and a finite cable. Assuming $x^{\prime}=0$, $z=z^{\prime}=0$ :

$$
r=\sqrt{x^{2}+y^{2}}
$$

and

$$
\begin{aligned}
\Phi(y) & =\frac{s_{0}}{4 \pi \sigma} \int_{L} \frac{d x}{\sqrt{x^{2}+y^{2}}} \\
& =\frac{s_{0}}{4 \pi \sigma} \ln \left|x+\sqrt{x^{2}+y^{2}}\right|_{-L / 2}^{L / 2} \\
& =\frac{s_{0}}{4 \pi \sigma}\left(\ln \left|\frac{L}{2}+\sqrt{\left(\frac{L}{2}\right)^{2}+y^{2}}\right|-\ln \left|-\frac{L}{2}+\sqrt{\left(\frac{L}{2}\right)^{2}+y^{2}}\right|\right) .
\end{aligned}
$$




\subsection{Infinite parallel membranes}

A closed solution for a pair of infinite parallel, passive, planar membranes embedded in a homogeneous conductive medium was found. The conductive medium is delimited by two parallel boundaries separated by distance $D$ and the membranes are separated by a distance $d$. This reduced problem is the simplest instance of the space/membrane equations and is useful for studying the time behavior of the equations. The problem is equivalent to the one-dimensional problem described in Figure 3.1(A). A "1D cell" is represented in a piecewise domain delimited by left and right membrane at points $x_{L}$ and $x_{R}$. An exterior electric field stimulus is implemented by Dirichlet conditions $\Phi_{D}^{0}=\Phi_{D}\left(x_{0}\right), \Phi_{D}^{1}=\Phi_{D}\left(x_{1}\right)$. The membrane voltages are $V_{m}^{L}=\Phi_{i}\left(x_{L}\right)-\Phi_{e}\left(x_{L}\right)$ and $V_{m}^{R}=\Phi_{i}\left(x_{R}\right)-\Phi_{e}\left(x_{R}\right)$. The membrane separation and domain length are $d$ and $D$ respectively.

Provided initial and boundary condition values, the problem has a unique solution in terms of the membrane voltage $V_{m}$. Following the notation of Fig.3.1(A) the membrane current can be written as

$$
I_{m}=-\sigma \frac{\Delta \Phi}{\Delta x}=-\sigma \frac{\Phi_{D}^{1}+V_{m}^{R}-V_{m}^{L}-\Phi_{D}^{0}}{D} .
$$

Assuming identical intra- and extra-cellular conductivities, currents in both sides of the membranes are equal. The two membrane voltages have the same magnitude and opposite sign. Defining $V_{m}=V_{m}^{R}=-V_{m}^{L}, \Phi_{D}=\Phi_{D}^{1}-\Phi_{D}^{0}$, and exterior current $I_{D}=-\sigma \frac{\Phi_{D}}{D}$ the membrane current is

$$
I_{m}=I_{D}-\frac{2 \sigma V_{m}}{D}
$$

The continuous membrane time evolution Eq. (2.41) is now

$$
C_{m} \frac{d V_{m}}{d t}=I_{D}-\frac{2 \sigma V_{m}}{D}-\frac{V_{m}}{R_{m}}
$$

Factorizing

$$
\frac{d V_{m}}{d t}+\left(\frac{1}{C_{m} D / 2 \sigma}+\frac{1}{C_{m} R_{m}}\right) V_{m}=\frac{I_{D}}{C_{m}} .
$$

This is a first order ordinary differential equation:

$$
\begin{aligned}
\frac{d V_{m}}{d t}+\frac{1}{\tau_{p}} V_{m} & =\frac{I_{D}}{C_{m}} \\
\tau_{p} & =\left(\frac{1}{C_{m} D / 2 \sigma}+\frac{1}{C_{m} R_{m}}\right)^{-1}
\end{aligned}
$$

whose solution is

$$
V_{m}(t)=c_{1} e^{-t / \tau_{p}}+\frac{I_{D}}{C_{m}} \tau_{p}
$$


With the initial condition $V_{m}(0)=0$, the unknown coefficient $c_{1}$ is $c_{1}=-\frac{I_{D}}{C_{m}} \tau_{p}$ producing:

$$
V_{m}(t)=\frac{I_{D}}{C_{m}} \tau_{p}\left(1-e^{-t / \tau_{p}}\right)
$$

A solution can also be obtained for the capacitive current

$$
I_{c}(t)=C_{m} \frac{\partial V_{m}(t)}{\partial t}=I_{D} e^{-t / t_{p}}
$$

and for the membrane current

$$
I_{m}(t)=I_{D} e^{-t / t_{p}}+\frac{V_{m}(t)}{R_{m}}
$$

These functions are quite informative about the time response of the membrane voltage and current to a pulse stimulus. Similar behavior is also observed in finite twodimensional and three-dimensional cells and in short cables. An example solution for this equation is presented in Fig. 3.1(B). Current densities on the infinite membrane are presented in the main figure, while the membrane voltage can be seen in the inset. At stimulus onset the capacitive current $I_{c}$ dominates, because the electric field simply passes through the membrane. As charges accumulate in both sides of the membrane $(t>0)$ the stimulus field is counteracted by the capacitor's own field, decreasing the current reaching the membrane. As the capacitance saturates and less and less charge can be stored, but the difference in charges polarity creates membrane voltage, enabling ionic flux. Ultimately, the membrane potential is so large that the entire injected current is carried by the ionic current across the membrane and the membrane capacitance is not charged further.

\subsection{Extracellular stimulation of a single cell}

The response of an infinitely long, cylindrical cell in three-dimensions to a traverse homogeneous field was presented by Krassowska and Neu [75] for a Beeler-Reuter ionic model [77] and later by Ying and Henriquez for a passive membrane model [37]. The problem is analyzed as a circular cell of diameter $d$ in two dimensions. Potentials for the cell under a homogeneous step field $E$ are

$$
\begin{aligned}
\Phi_{i}(r, \theta, t) & =-a(t) \cdot E r \cos \theta \quad r<d / 2 \\
\Phi_{e}(r, \theta, t) & =-E r \cos \theta-b(t) \cdot E \frac{d^{2}}{4 r} \cos \theta \quad r>d / 2 \\
V_{m}(\theta, t) & =E d \cos \theta\left(1-e^{-\frac{t}{\tau}}\right)(1-\epsilon)=E d \cos \theta\left(1-\left(e^{-\frac{t}{\tau}}+\left(1-e^{-\frac{t}{\tau}}\right) \epsilon\right)\right) .
\end{aligned}
$$



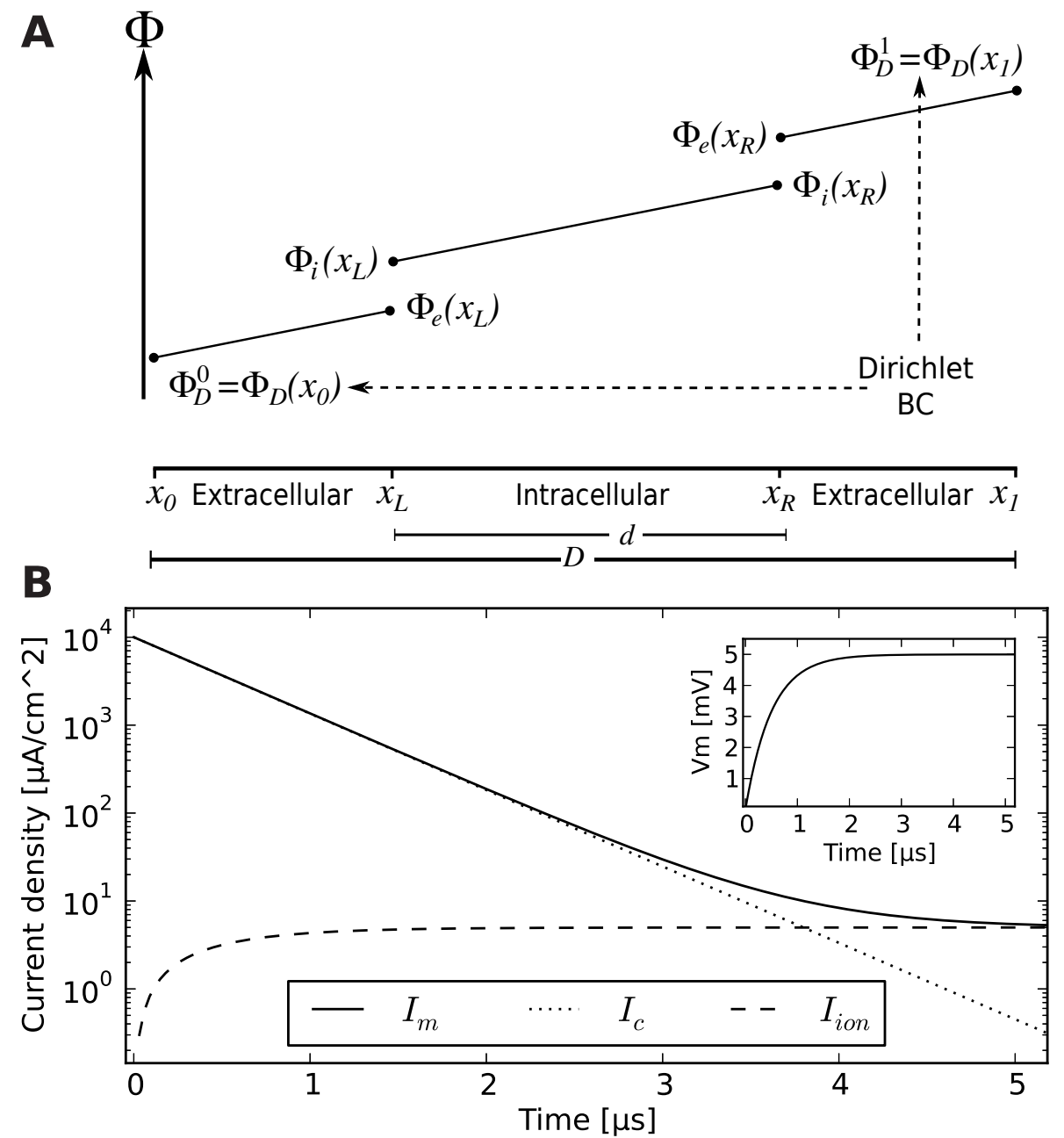

Figure 3.1: Analytic solution of the infinite parallel membranes problem. (A) Illustration of the geometry used to derive the relation between membrane current and voltage of Eq. (3.1). (B) Analytic solution of the 1D Problem. 
With

$$
\begin{aligned}
a(t) & =\frac{2 \sigma_{e}}{\sigma_{i}+\sigma_{e}}\left\{e^{-\frac{t}{\tau}}+\left(1-e^{-\frac{t}{\tau}}\right) \epsilon\right\} \\
b(t) & =1-\frac{2 \sigma_{i}}{\sigma_{i}+\sigma_{e}}\left\{e^{-\frac{t}{\tau}}+\left(1-e^{-\frac{t}{\tau}}\right) \epsilon\right\} \\
\tau & =\frac{1}{\frac{1}{C_{m} R_{m}}+\frac{2 \sigma_{i} \sigma_{e}}{C_{m} d\left(\sigma_{i}+\sigma_{e}\right)}}, \epsilon=\frac{\tau}{C_{m} R_{m}}
\end{aligned}
$$

Assuming $\sigma_{i}=\sigma_{e}$

$$
\tau_{s c}=\left(\frac{1}{C_{m} R_{m}}+\frac{\sigma_{i}}{C_{m} d}\right)^{-1}
$$

The left term in the sum is the inverse of the membrane time constant $\tau_{m}=C_{m} R_{m}$. The right term is the inverse of the so called "cell time constant" [75]:

$$
\tau_{c}=\frac{C_{m} d}{\sigma_{i}}
$$

The overall time constant for the single cell $\tau_{s c}$ is seen to be related to these two. Respectively, they govern the step response of the cell as determined by the intracellular resistivity/membrane capacitance circuit and the membrane resistance/membrane capacitance circuit. The cell constant is clearly smaller than the cell time constant and dominates the value of $\tau_{s c}$. Some typical values for the above parameters are $C_{m}=1 \mu \mathrm{F} / \mathrm{cm}^{2}, \sigma_{e}=\sigma_{i}=10 \mathrm{mS} / \mathrm{cm}, d=10 \mu \mathrm{m}, R_{m}=1 \mathrm{mS} / \mathrm{cm}^{2}$. A typical membrane constant would be $\tau_{m}=1 \mathrm{~ms}$ and the cell constant $\tau_{c}=0.1 \mu \mathrm{s}$ implying $\tau_{s c} \approx 0.1 \mu \mathrm{s}$. The cell time constant is much shorter, implying a major contribution to the overall constant in (3.6) for cells in the 1-10um diameter range. Traverse stimulation of these cells reacts quite rapidly and this will have major implications in the numerical solution of the space/membrane equations. In that case, a similar relation was found for the stability restrictions with the typical size of a mesh subdivision. The results presented for the equivalent $2 \mathrm{D}$ cell in this section also extend to three dimensions where a similar time constant is found for spherical cells [76].

\subsection{Cable solution for extracellular stimulation}

An analytic solution for a cable with sealed ends under a homogeneous extracellular parallel step field was provided by Cartee and Plonsey [60] and extended by Monai and colleagues for arbitrary fields [74]. Green's function method was used in [74] to find the series solution to Eqs. 2.61 and 2.62, which reads:

$$
V_{m}(x, t)=\sum_{n=0}^{\infty} A_{n}(x) I_{s} \tau_{n}\left(1-\exp \left(-\frac{t}{\tau_{n}}\right)\right)
$$


with $I_{s}$ the total stimulus current ${ }^{1}$, the amplitude term

$$
A_{n}(x)=\frac{r_{e} \lambda^{2}}{\tau_{m} \alpha_{n, n}}\left(\varphi_{n}(L)-\varphi_{n}(0)\right) \varphi_{n}(x),
$$

eigenfunctions $\varphi_{n}(x)=\cos \left(\mu_{n} x\right)$, eigenvalues $\mu_{n}=\frac{n \pi}{L}$, eigenfunctions inner products $\alpha_{n, n}=\frac{L}{2}$, and a family of time constants

$$
\tau_{n}=\frac{1}{\frac{1}{\tau_{m}}+\frac{\mu_{n}^{2} \lambda^{2}}{\tau_{m}}} .
$$

The behavior of this solution for short cables is illustrated in Figure 3.2.

The cell time constant is also implicit in $\tau_{n}$. Note that with $\lambda^{2}=\frac{r_{m}}{r_{i}}$ and $\tau_{m}=r_{m} c_{m}$

$$
\tau_{n}=\frac{\tau_{m}}{1+\frac{n^{2} \pi^{2} \lambda^{2}}{L^{2}}}=\left(\frac{1}{\tau_{m}}+n^{2} \frac{\pi^{2}}{L^{2} c_{m}} \frac{1}{r_{i}}\right)^{-1} .
$$

Replacing $r_{i}=4 / \sigma_{i} \pi d^{2}$ and $c_{m}=\pi d C_{m}$

$$
\tau_{n}=\left(\frac{1}{\tau_{m}}+n^{2} \frac{\pi^{2}}{L^{2}} \frac{d \sigma_{i}}{4 C_{m}}\right)^{-1},
$$

a term similar to the cell time constant (3.7) appears in the $n$-independent term

$$
\tau_{c c}=\frac{C_{m} 4 L^{2}}{\sigma_{i} d \pi^{2}}
$$

In terms of this "cable-cell time constant" $\tau_{c c}$ the family of time constants is now

$$
\tau_{n}=\left(\frac{1}{\tau_{m}}+n^{2} \frac{1}{\tau_{c c}}\right)^{-1}
$$

and some values are

$$
\begin{aligned}
\tau_{0} & =\tau_{m} \\
\tau_{1} & =\left(\frac{1}{\tau_{m}}+\frac{1}{\tau_{c c}}\right)^{-1} \\
\tau_{2} & =\left(\frac{1}{\tau_{m}}+4 \frac{1}{\tau_{c c}}\right)^{-1} \\
\tau_{3} & =\left(\frac{1}{\tau_{m}}+9 \frac{1}{\tau_{c c}}\right)^{-1} \\
\tau_{4} & =\left(\frac{1}{\tau_{m}}+16 \frac{1}{\tau_{c c}}\right)^{-1} \\
& \vdots \\
\tau_{\infty} & =0 .
\end{aligned}
$$

\footnotetext{
${ }^{1}$ The relation of the total stimulus current to the electric field was not provided in Monai et al. [74]. Using Plonsey's core conductor model this can be calculated as $I_{s}=E / r_{e}$ with $E$ the magnitude of the electric field, $r_{e}=4 / \sigma_{e} \pi D^{2}$ the extracellular axial resistance and $D$ the diameter of the extracellular core conductor. Usually $D$ can be a very large value which after some point looses its influence on $V_{m}$. See Plonsey and Barr for a description of the core-conductor model (p. 40, 156, 228 [47]).
} 
This family of time constants shows that in general, the response of the finite cable to stimulation cannot simply be determined by the membrane time constant $\tau_{m}$ but it is still related to the cell constant.

For typical cases of a cable with finite length near the length constant $\lambda$, the cell constant term is dominant. First of all, notice that for this particular solution of $V_{m}$, the amplitude term $A_{n}(x)$ equals zero for $n=\{0,2,4,6, \ldots\}$. This results from the eigenfunction sum $\varphi_{n}(L)-\varphi_{n}(0)$ canceling out for even $n$. This implies that $\tau_{m}, \tau_{2}, \tau_{4}, \ldots$ do not contribute to the solution. For values similar to the previous section $\left(C_{m}=1 \mu \mathrm{F} / \mathrm{cm}^{2}, \sigma_{i}=10 \mathrm{mS} / \mathrm{cm}, d=1 \mu \mathrm{m}, R_{m}=1 \mathrm{mS} / \mathrm{cm}^{2}\right.$ and a short cable $L=80 \mu \mathrm{m}$ as in Fig. 3.2) the time constants take the value $\tau_{m}=1 \mathrm{~ms}, \tau_{c c}=26 \mu \mathrm{s}$ and the first five family values are $\tau_{0}=1 \mathrm{~ms}, \tau_{1}=25 \mu \mathrm{s}, \tau_{2}=6.5 \mu \mathrm{s}, \tau_{3}=2.9 \mu \mathrm{s}$, and $\tau_{4}=1.6 \mu \mathrm{s}$. As $\tau_{0}$ is irrelevant, the time constant of the response is largely determined by $\tau_{c c}$ and the first few values of $\tau_{n}$ (their influence decreases for larger $n$ because $V_{m} \propto \tau_{n}$ ).

The membrane time constant only recovers its influence on $\tau_{n}$ when $L \gg \lambda$. This can be seen for instance in Eq. (3.9) where a growing $L$ brings the denominator close to 1 . For an infinitely long cell $L \rightarrow \infty, \tau_{c c} \rightarrow \infty$ and $\tau_{n} \approx \tau_{m}$ is constant in Eq. (3.8), leaving the sum. The time evolution is then given by the membrane time constant only.

\subsection{Current injection and discharge of a spherical cell}

The next equations provide a practical way to calculate the voltage and potential around a spherical cell. These values will be useful for comparison and validation of potential and membrane current values in the following chapters. First, the membrane current density relation to potential is discussed. The total current passing the passive membrane of a cell during current injection must amount for the total current being injected as shown by the membrane current continuity Eq. (2.53). A spherical cell can be modeled as a spherical current source of radius $R$ and area $A=4 \pi R^{2}$. In a medium of conductivity $\sigma_{e}$, the potential at a point distance $r$ is given by [33, 78]:

$$
\Phi(r)=\frac{R^{2} J}{\sigma_{e} r} \text { for } r>R
$$

with $J$ the current surface density

$$
J=\frac{I}{4 \pi R^{2}}
$$

and $I$ the total current injected. An example value of the extracellular potential measured around the spherical cell is now provided. At the peak rising rate of an action potential, a cell can emit up to $1000 \mu \mathrm{A} / \mathrm{cm}^{2}$ of current. For a medium with conductivity $10 \mathrm{mS} / \mathrm{cm}$ and a $10 \mu \mathrm{m}$ diameter cell, $R=5 \mu \mathrm{m}$ and a the potential near the cell is

$$
\Phi(r)=\frac{(0.0005 \mathrm{~cm})^{2} 1000 \frac{\mu \mathrm{A}}{\mathrm{cm}^{2}}}{10 \frac{\mathrm{mS}}{\mathrm{cm}} r}=\frac{25 \times 10^{-6}}{r} \mathrm{mV} \cdot \mathrm{cm}
$$

At distances of 10 and $100 \mu \mathrm{m}$ this produces potentials of 0.025 and $0.0025 \mathrm{mV}$ respectively, which are typical ranges for extracellular recordings. 


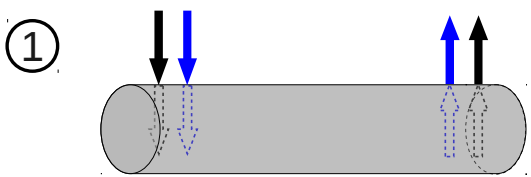

Stimulus onset. Current inside the cell is mostly capacitive. Membrane current equals capacitive current.
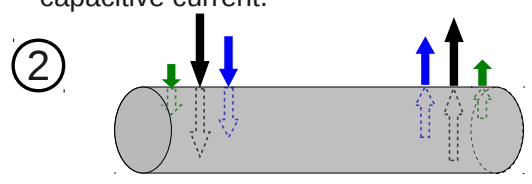

Potential difference enables ionic current. Membrane current is the sum of capacitive and ionic current.

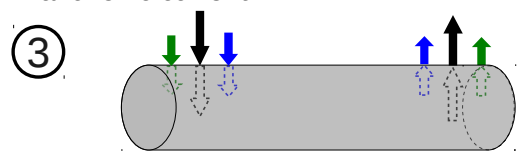

Capacitive current settles as the capacitance starts to saturate. Ionic current keeps growing and now equals capacitive current.

\section{(4)}

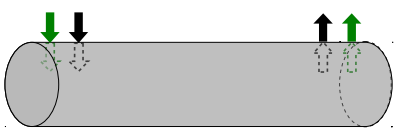

Capacitive current stops. Steady state is reached. Membrane current is the ionic current.

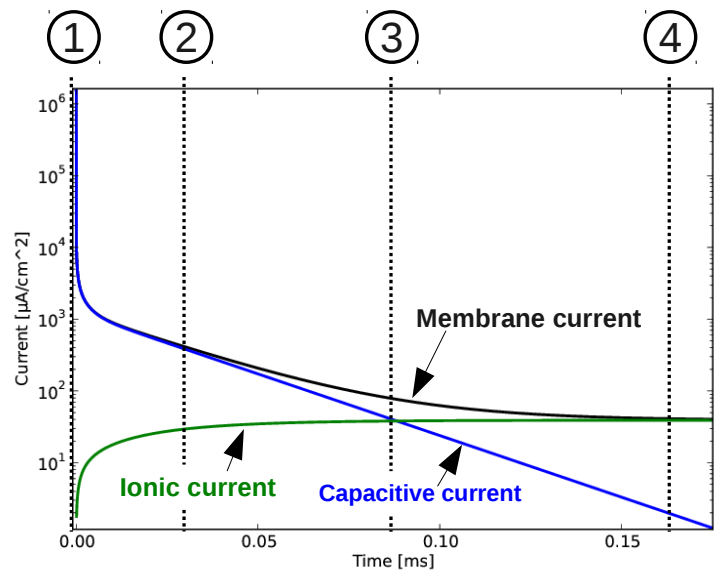

Membrane voltage $\left(V_{m}\right)$

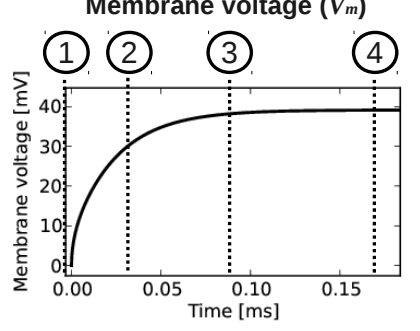

Figure 3.2: Illustration (left) and analytic solution (right) of the response of a short neuronal cable ( $80 \mu \mathrm{m}$ long, $1 \mu \mathrm{m}$ diameter, $1 \mu \mathrm{F} / \mathrm{cm}^{2}$ membrane capacitance, $100 \mathrm{Ohm}^{*} \mathrm{~cm}$ intra and extra-cellular resistivity, $1 \mathrm{kOhm} * \mathrm{~cm}^{2}$ membrane resistance) to a homogeneous extracellular stimulation pulse $\left(1 \times 10^{4} \mathrm{mV} / \mathrm{cm}\right)$. Currents and voltages are measured at the right tip of the cable. Arrows indicate the direction of membrane currents flow (largest at the cable boundaries) and the colors follow the traces in the right. Notice that in the cable equation approximation, the cable has no transversal area so theoretically, current does not flow through the caps of this "cylinder". 
The charging of the membrane voltage for the same cell is given by [47]

$$
V_{m}(t)=I \frac{R_{m}}{4 \pi R^{2}}\left(1-e^{-t / \tau_{m}}\right)
$$

Provided that stimulation has stopped and a certain initial potential $V_{0}$ is reached, the cell will proceed to discharges as [47]

$$
V_{m}(t)=V_{0} e^{-t / \tau_{m}}
$$

The discharge follows the membrane time constant. The current density behavior during discharge is given by a negative capacitive current decaying negatively to zero and a positive ionic current also decaying to zero with the same time constant. Note that the membrane current $I_{m}$ for this case is zero indicating no potential gradients inside the cell. For a cell to produce a membrane current and thus an extracellular potential in this situation, lateral or external currents would be needed (e.g. by a cell process). The limit case of a membrane with infinite resistance would imply the cell wont discharge, retaining the initial potential. If the membrane resistance was zero the cell would immediately discharge.

\subsection{Summary}

Solutions for analytically treatable geometrical configurations of the space/membrane equations were presented. These solutions are provided as a summary of previous works and also as a reference for further chapters. When no cells are present and a homogeneous medium is considered, potential can be calculated easily from the current sources distribution. Moreover, the simplest case of membrane presence can be studied in the infinite parallel membranes case presented in Section 3.3. This problem provides an easy to visualize case and reveals important properties of the response of the cell to extracellular stimulation. The response of a quasi two dimensional cell to extracellular stimulation was then presented and will be crucial for the stability analysis of Chapter 5 .

The cable solution of Section 3.5 showed how, the typical neuronal cable behaved under extracellular stimulation and that, in the analysis of the cable equation the membrane time constant is not the only parameter of the time response. Finally, the chapter focuses in the reaction of a spherical passive cell to intracellular injection which is required to study the extracellular signature of the action potential in Chapter 7 .

A common theme in the analytical solutions of Sections 3.4 and 3.5 is the emergence of the cell time constant parameter. The cell time constant is different from the membrane time constant and typically smaller in value. As this parameter dominates the time response, the time evolution of the cell response to extracellular potentials is largely affected by it and has an important influence in the numerical treatment of the problem. As it will be seen in the next chapters, an explicit time solution algorithm finds trouble if the iteration time step is larger than the cell time constant of the individual grid elements. Implicit algorithms are necessary to deal with this restriction. 


\section{Discretization and numerical strategy}

\subsection{Introduction}

The space/membrane equations describe the spatio-temporal evolution of potential in conductive media and across the cell membrane. An alternative to these equations is the reduced cable equation but this assumes that the cell lives in an homogeneous, isopotential medium. Meanwhile cells are quite often found in heterogeneous media, surrounded by other cells and enclosed by a number of boundary conditions. Real cells and particularly neurons, also exhibit complex three-dimensional morphologies. The space/membrane equations can model such configurations but only for few cases with high symmetry, an analytic solution can be derived (see for instance previous chapter). Solving the space/membrane equations for arbitrary geometries demands numerical methods.

Solution to the partial differential equations (2.54) and (2.55) for arbitrary geometries requires then numerical treatment and techniques such as the finite difference method (FDM), the boundary element method (BEM), the finite volume method (FVM), or the finite element method (FEM). Solution to the time dependent equation (2.41) requires explicit or implicit numerical time integration schemes.

Although very few attempts have been made to produce computer solvers for these specific equations, two other research areas: hearth electrophysiology and electroporation, have dealt with similar problems. The main concern of heart electrophysiology modeling is the simulation of bundles of muscle cells or myocites and a set of partial differential equations (PDEs) similar to the space/membrane equations is used. These equations are known as the bidomain equations which model cell bundles and the space between them into a homogenous overlap of intra- and extra-cellular space [79, 80]. In the bidomain the single cell individual membrane is not explicitly modeled.

In electroporation cells are exposed to strong electric fields to increase their electrical conductivity and permeability to external substances. In cell electroporation modeling, equations similar to the space/membrane equations and in a few studies, numerical PDE solvers have been used to model arbitrarily shaped cells. Although those solvers exist, no ideal method was found in this area with the ability to model the detailed three-dimensional neuronal membrane and the extra/intra-cellular space.

Before the strategy presented in this chapter and the tool presented in Chapter 6 were developed, a complete and fast solver of the full space/membrane equations was missing. Previously existing works had a number of limitations. In their numerical approaches, two main drawbacks were recognized: computational restrictions to represent 
detailed geometries and the lack of efficient time evolution schemes. The use of commercial numerical tools has also hindered the development of these methods but this will be discussed further in Chapter 6 . Previous attempts to solve the space/membrane equations are now listed.

\subsubsection{Previous work}

Methods related to the finite difference method, namely the equivalent circuit method $[81,82]$ and the transport lattice method $[83,84]$ divide the cell in discrete electrical circuit components and were introduced to easily calculate the current and potential on and around the cell in electroporation. These methods are limited in the geometries they can describe, as discrete pieces of the intracellular, extracellular space and membrane have to be explicitly described to form circuits of capacitors and resistors.

BEM approaches to solve this equations were developed in the early 1990's [85, 86] providing fast computation times. Although computationally more efficient, the BEM is generally limited to cylindrical symmetry, requires a careful design depending on the number of cells, and cannot represent spatially heterogeneous conductivities. Finite difference method approaches such as the one described by See and colleagues [51] represent geometries in meshes with a fixed-everywhere grid spacing determined by the smallest feature in the domain. Due to the very large number of elements, the computation time of these simulations would be impractically long for realistic, submicrometer morphological features.

The finite element method allows representing more detailed geometries as the discretization can be irregular and adapted to have higher resolutions in regions of interest. Early FEM models for cell stimulation were able to describe the potentials around single and multiple cells in a conducting bath $[87,88]$ but these were not time dependent. FEM [35, 37, 39, 52] and finite volume methods [38, 53] have been employed in similar cases, but these have been limited to unrealistic spatial scales [38, 53], restrictive time integration schemes [35, 37, 38, 53, 52], two dimensions [37], and the absence of ion channels $[35,37,38,52]$.

In the interest of modeling electrode/cell configurations, models such as the thinfilm approximation have been used. The thin-film approximation supposedly models the electrical and thermal resistance in the interface between two solids. Moulin et al. adapted this method to represent the capacitance and conductance of the cell membrane [39]. The continuity of current between the extracellular and intracellular space was preserved through Neumann boundary conditions. Although interesting, the technical details of this method belong to COMSOL, Inc. The technical document cited by Moulin et al. was not accessible at the writing of this work.

Specialized FEM models to represent gap junctions by Fear and Stuchly [35] and electroporation by Pucihar et al. [52] can model more detailed two- and three-dimensional time dependent geometries. Although these models consider finer details, they were not developed for large time simulations. None of these works described the time discretization strategy used. Both Fear and Stuchly and Pucihar et al. used commercial tools to solve the equations.

In the two-dimensional work of [37] the time strategy is clearly specified as an explicit 
forward Euler method. With the forward Euler method applied on grids with submicron elements, extremely fine timesteps have to be used for numerical stability (see Chapter 5). As a result, overall computation time is impractically long for sub-micron geometries. An accelerated time strategy was presented in [51] but is limited to box elements in a FDM scheme. The scheme of Xylouris [89] included Newton's method for the solution of the non-linear ionic currents. This scheme imposes scalability restrictions as the number of channels increases.

As a side note, except for the cable equation, experimental validation of the numerical methods for the solution of the space/membrane equations is scarce. An attempt to compare the result of simulations to experimental results was made by Pucihar et al. [90]. In this study numerical calculations where compared to experimental measurements of homogeneous field cell stimulation with a potential sensitive dye. The calculated steady state potential was found to match the observed dye response. However this model did not consider membrane capacitance. Neuronal cable solvers can also be considered as solvers of the space/membrane equations and enough experimental evidence suggests it is accurate. However cable solvers do not model the extracellular space. Cable solvers will be discussed again at the end of this chapter.

In the next sub-sections the preferred method for solution of the space/membrane equations, the FEM, is presented and the solution strategy used is discussed. In the rest of the chapter the strategy is finely detailed. This level of detail was fundamental for the implementation of the computer software described in Chapter 6 .

\subsection{The finite element method}

The finite element method (FEM) was chosen as the solution method for the space/membrane equations. The FEM provides a robust numerical algorithm for approximating the solution of partial differential equations in complex geometries. The FEM subdivides the problem in small sub-domains named "elements" whose individual solutions match accordingly to provide a global, consistent solution. The method's "plan of attack" can be roughly divided in four steps:

- First, the problem is formulated in its so called "weak form". A problem in weak form is not required to satisfy an absolute solution but a "weak" one, i.e. a solution that partially satisfies the problem's equation even when its derivatives might not exist (e.g. local regions of the solution are not smooth enough).

- Second, the solution domain is divided in its discrete version, that is, a collection of discrete elements and the weak form is transferred to this finite dimensional space. A common form is the division of the spatial domain in triangles for twodimensional problems or tetrahedra for three-dimensional problems ) although other geometries can be used.

- Third, the finite dimensional version of the problem is converted to an equivalent algebraic form in a process known as assembly. In the algebraic form, the problem is equivalent to a linear system of equations where the value of the solution at the element vertices are the unknowns. The problem parameters and terms associated 


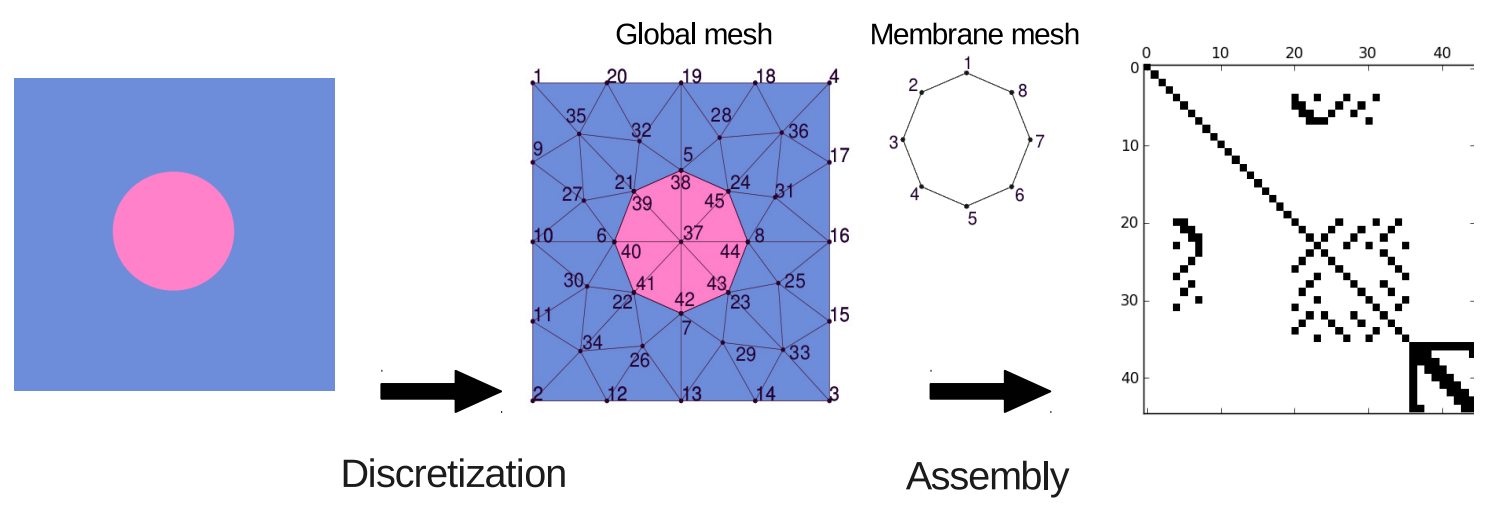

Figure 4.1: The finite element algorithm for a cell (light red) and extracellular space (blue) geometry. A special condition in this problem is that the membrane will represent an additional mesh. The right most figure represents the non zero elements of the coefficient matrix.

to the partial differential equation typically form the left-hand coefficient matrix and the boundary conditions the right hand side of the system.

- The linear system is solved with an appropriate linear solver whose choice depends on the spectral properties of the system.

See Figure 4.1 for an illustration of the method in cell electrophysiology.

The FEM provides just an approximation to the solution of the differential equation in the discrete domain. The FEM was developed in the middle of the 20th century where elasticity and structural analysis applications prompted for design and simulation methods that could reduce experimentation costs [91]. Well known works provide a mathematical treatment of the method, such as Ciarlet's book [92]. The work presented in this chapter required one of the most basic FEM forms. A very practical approach to the finite element method and a valuable guide during this thesis was found in the work of Olver [93, 94].

\subsection{Solution strategy}

A numerical strategy that separates the spatial potential problem from the time dependent, membrane currents problem is used to solve the space/membrane equations. Treating the space/membrane equations involves the solution of a linear parabolic partial differential equation for the potential in space and a time iteration method for the solution of the membrane current. Treatment of the membrane currents can also involve the solution of a large set of non-linear ordinary differential equations that represent the ionic current kinematics. Ideally, the solution of the entire system of equations would be solved simultaneously, considering all the variables involved, and using an implicit time discretization method. This is however impractical for large problems. Three main aspects prompt for alternative methods: 
- The piecewise nature of the problem, imposed by the capacitive and conductive cell membrane, brings a strong discontinuity on the solution domain and requires special treatment.

- The frequent non-linearity of the equations describing the ionic currents implies that non-linear solution methods would be needed. Methods such as the Newton-Raphson could be used but the mathematical treatment would be cumbersome and its solution computationally expensive.

- Besides the spatial potential and membrane current equations, each ionic current can imply two or more ordinary differential equations. Modern neuron descriptions requiring 10 or more ionic currents [40] would imply simultaneous implicit solution of tens of variables for each finite element and a considerably large Jacobian matrix in the case of the Newton-Raphson method.

Thankfully, as a consequence of the quasi-static approximation, most of the solution domain is time independent. As charge decay only occurs in the membrane, temporal processes do not occur inside the intracellular or extracellular space. The potential can be calculated statically. Time iteration is only relevant in the membrane so a smaller problem can be defined for the membrane domain alone. The spatial potentials can be solved independently for a fixed time while the time iteration can be done assuming the potentials remain constant. From the space/membrane equations the problem only needs to be expressed in terms of a potential and membrane current variable. Solution of these two should, at the same time, satisfy the constrains imposed by the exterior boundary conditions and the membrane voltage. Moreover this separation simplifies the implementation of the solution software, allowing modularity between the different solution algorithms.

The handling of discontinuities in the spatial domain is a know as "domain decomposition" in the applied mathematics literature and extensive work exists in this area [95, 96]. An specific approach together with a discretization and proof of existence of the solution for the spatial problem in Eqs. (2.42), (2.39) and (2.40) was presented by Wohlmuth and Lamichhane [71, 97]. This approach was selected as the solution approach ${ }^{1}$.

After an spatial solution is obtained for the boundary value problem, the membrane voltage has to be integrated over time. At least one more approach for solving the space/membrane equations uses this alternative [37]. This solution scheme is logical and shares some similarities with bidomain solvers [98, 54, 99]. Once the spatial problem is separated from the time problem, explicit or implicit methods can be used for the time discretization [100].

Explicit methods as the forward Euler method or implicit methods as the backward Euler or the Crank-Nicolson method [55] can be used. The forward Euler was the first

\footnotetext{
${ }^{1}$ Wohlmuth and Lamichhane method allows for a spatial discretization in which the elements describing the domains are non-matching, that is, that they do not necessarily share common nodes on the interface between domains. The implementation in this thesis assumed the interface nodes matched simplifying the implementation. The use of non-matching grids was considered unnecessary for the presented work, however the solution algorithm could be extended in the future it that was needed.
} 
attempted method because it is easy to implement. This method is limited as will be presented in Chapter 5 and brings strict restrictions in the time steps. Ying et al. opted for an explicit method [37]. The more favorable, implicit Crank-Nicolson was implemented next and demonstrated to be more robust for large timesteps. Also, a solution strategy was found that employs an algebraic structure not much more complicated than in the forward Euler method. The Crank-Nicolson scheme allows simulation time-steps orders of magnitude larger than the Euler scheme, without causing numerical instabilities. The use of this implicit method also grants applicability of adaptive time step schemes, shortening computation times by one to two orders of magnitude. This will be proven in Chapter 7 .

\subsubsection{Solution algorithm}

To solve the complete space and membrane problem, the equations governing the potentials in the intra- and extracellular domains and the equations governing the time development of the membrane currents are solved in an alternating loop:

- Poisson's Eqs. (2.54), (2.55), for the potential and the current continuity Eq. (2.53) with boundary conditions (2.51) and (2.52) and a fixed $V_{m}$ are solved with the FEM for a given moment in time. In this step, the system of partial differential equations is solved to obtain variables $\Phi_{e, i}$ at each of the domain finite elements and finding $I_{m}=\mathbf{n}_{e} \cdot \sigma_{e} \nabla \Phi_{e}=-\mathbf{n}_{i} \cdot \sigma_{i} \nabla \Phi_{i}$ at each of the elements touching the membrane. At $t=0$ the potential and membrane current take the values of the initial conditions.

- After this, the membrane currents Eq. (2.41) is integrated using the newly calculated membrane current $I_{m}$. The ionic currents are solved using the previous value of $V_{m}$ i.e. the one used in the first part of the algorithm. After this step is completed, a new value for $V_{m}$ is found.

Appendix Chapter $\mathrm{G}$ presents a more concrete version of this algorithm as script code. In the next sections the spatial and temporal discretizations are detailed.

\subsection{Space discretization}

\subsubsection{Weak formulation}

\subsubsection{Spatial potential problem}

The weak finite element formulation is obtained by multiplying equations (2.54) (and identically the intracellular Eq. (2.55)):

$$
-\nabla \cdot \sigma_{e} \nabla \Phi_{e}=s_{e}
$$

by test functions $v_{e}$ (and $v_{i}$ ), multiplying and integrating on both sides,spatial potential problem

$$
-\int_{\Omega_{e}}\left(\nabla \cdot \sigma_{e} \nabla \Phi_{e}\right)\left(v_{e}\right) d V=\int_{\Omega_{e}} s_{e}\left(v_{e}\right) d V .
$$


Green's first identity can be applied to the left hand side:

$$
\int_{\Omega_{e}}\left(\sigma_{e} \nabla \Phi_{e}\right) \cdot\left(\nabla v_{e}\right) d V-\int_{\partial \Omega_{e}}\left(\nabla \Phi \cdot \mathbf{n}_{e}\right)\left(v_{e}\right) d S=\int_{\Omega_{e}} s_{e}\left(v_{e}\right) d V .
$$

The extracellular space $\Omega_{e}$ boundary $\partial \Omega_{e}$ can be divided in three parts: a region of the exterior boundary where Dirichlet boundary conditions apply $\partial \Omega_{D}$, a region of the exterior boundary where Neumann boundary conditions apply $\partial \Omega_{N}$, and the membrane part of the boundary $\Gamma$. The second term in the left hand side can then be separated in three integrals

$$
\begin{aligned}
& \int_{\Omega_{e}}\left(\sigma_{e} \nabla \Phi_{e}\right) \cdot\left(\nabla v_{e}\right) d V-\int_{\Gamma}\left(\sigma_{e} \nabla \Phi_{e} \cdot \mathbf{n}_{e}\right)\left(v_{e}\right) d S- \\
& \int_{\partial \Omega_{D}}\left(\sigma_{e} \nabla \Phi_{e} \cdot \mathbf{n}_{e}\right)\left(v_{e}\right) d S-\int_{\partial \Omega_{N}}\left(\sigma_{e} \nabla \Phi_{e} \cdot \mathbf{n}_{e}\right)\left(v_{e}\right) d S=\int_{\Omega_{e}} s_{e}\left(v_{e}\right) d V .
\end{aligned}
$$

The Dirichlet section of the integral vanishes ${ }^{2}$ as the FEM requires $v_{e}=0$ on $\partial \Omega_{D}$ so

$$
\int_{\Omega_{e}}\left(\sigma_{e} \nabla \Phi_{e}\right) \cdot\left(\nabla v_{e}\right) d V-\int_{\Gamma} I_{m} v_{e} d S-\int_{\partial \Omega_{N}} I_{N} v_{e} d S=\int_{\Omega_{e}} s_{e} v_{e} d V .
$$

Moving the unknowns to the left hand side produces

$$
\int_{\Omega_{e}} \sigma_{e} \nabla \Phi_{e} \cdot \nabla v_{e} d V-\int_{\Gamma} I_{m} v_{e} d S=\int_{\partial \Omega_{N}} I_{N} v_{e} d S+\int_{\Omega_{e}} s_{e} v_{e} d V .
$$

The intracellular version of the equation assumes than no cell is open to the exterior boundary and uses the opposite normals on the membrane integral

$$
\int_{\Omega_{i}} \sigma_{i} \nabla \Phi_{i} \cdot \nabla v_{i} d V+\int_{\Gamma} I_{m} v_{i} d S=\int_{\Omega_{i}} s_{i} v_{i} d V
$$

Note that the interface condition on $\Gamma$ is comparable to a Neumann boundary condition, with the difference that in this model $I_{m}$ is an unknown function on membrane space.

Combining (4.1) and (4.2) produces

$$
\int_{\Omega} \sigma \nabla \Phi \cdot \nabla v d V+\int_{\Gamma} I_{m}\left(v_{i}-v_{e}\right) d S=\int_{\partial \Omega_{N}} I_{N} v d S+\int_{\Omega} s v d V
$$

Here, $\sigma, \Phi, v$ represent the conductivity, potential and test function in any of the non-overlapping domains $\Omega_{e, i}$. The bracket operator []$_{i, e}$ is introduced to change the

\footnotetext{
${ }^{2}$ Note that although the Dirichlet part disappears now as required by the finite element method, it has to be considered in the matrix assembly by making the boundary node values known variables with the Dirichlet values. This is a tricky part of the method but I refer to other works for an explanation.
} 
sign of the operand according to the face of $\Gamma$ that is being evaluated

$$
[f(\mathbf{x})]_{i, e}= \begin{cases}-f(\mathbf{x}) & \mathbf{x} \text { in } \Gamma_{i} \\ f(\mathbf{x}) & \mathbf{x} \text { in } \Gamma_{e}\end{cases}
$$

The spatial potential problem is, with this notation

$$
\int_{\Omega} \sigma \nabla \Phi \cdot \nabla v d V+\int_{\Gamma} I_{m}[v]_{e, i} d S=\int_{\partial \Omega_{N}} I_{N} v d S+\int_{\Omega} s v d V .
$$

Integration is performed in the intracellular and extracellular face of $\Gamma$.

\subsubsection{Membrane voltage condition}

The membrane voltage condition (2.36) is expressed in the weak form as

$$
\int_{\Gamma}\left(\Phi_{i}-\Phi_{e}\right) u d S=\int_{\Gamma} V_{m} u d S
$$

with $u$ a test function, also restricted to membrane space. Eq. (4.4) can be understood as a special Dirichlet boundary condition where the values must comply with a fixed potential difference $V_{m}$. With the bracket operator this translates to

$$
\int_{\Gamma}[\Phi]_{e, i} u d S=\int_{\Gamma} V_{m} u d S
$$

The complete system of spatial equations is formed by Eqs. (4.3) and (4.4).

\subsubsection{Matrix form of the problem}

The matrix form of the problem is obtained by choosing two set of piecewise linear basis functions:

- $\left\{v^{1}, v^{2}, \ldots, v^{N_{\Omega}}\right\}$ for each of the $N_{\Omega}$ nodes in a triangulation over the solution domain $\Omega$ namely $\Omega^{h}$, and

- $\left\{u^{1}, u^{2}, \ldots, u^{N_{\Gamma}}\right\}$ for each of the $N_{\Gamma}$ nodes in a triangulation over the solution domain $\Gamma$ namely $\Gamma^{h}$.

Here $h$ refers to the typical length of the triangulation. The result is a collection of $M=N_{\Omega}+N_{\Gamma}$ equations,

$$
\begin{aligned}
\int_{\Omega} \sigma \nabla \Phi \cdot \nabla v^{\mathrm{i}} d V+\int_{\Gamma} I_{m}\left[v^{\mathrm{i}}\right]_{e, i} d S & =\int_{\Omega} s v^{\mathrm{i}} d V \quad \mathrm{i}=1, . ., N_{\Omega}, \\
\int_{\Gamma} u^{\mathrm{j}}[\Phi]_{e, i} d S & =\int_{\Gamma} u^{\mathrm{j}} V_{m} d S \quad \mathrm{j}=1, . ., N_{\Gamma} .
\end{aligned}
$$


For simplicity the Neumann boundary condition term $\int_{\partial \Omega_{N}} I_{N} v d S$ was dropped but can be added in any future step as a known value.

Let $\Phi^{\mathrm{k}}, I_{m}^{\mathrm{j}}$, and $V_{m}^{l}$ be the approximations of values $\Phi\left(x^{\mathrm{k}}\right), I_{m}\left(x^{\mathrm{j}}\right)$ and $V_{m}\left(x^{l}\right)$ at triangulation nodes $x^{\mathrm{k}} \in \Omega^{h}, x^{\mathrm{j}} \in \Gamma^{h}$ and $x^{\mathrm{l}} \in \Gamma^{h}$. The continuous functions can be approximated by

$$
\begin{aligned}
\Phi & \approx \sum_{\mathrm{k}=1}^{N_{\Omega}} \Phi^{\mathrm{k}} v^{\mathrm{k}}, \\
I_{m} & \approx \sum_{\mathrm{j}=1}^{N_{\Gamma}} I_{m}^{\mathrm{j}} u^{\mathrm{j}}, \text { and } \\
V_{m} & \approx \sum_{\mathrm{l}=1}^{N_{\Gamma}} V_{m}^{\mathrm{l}} u^{\mathrm{l}},
\end{aligned}
$$

and the potential gradient by

$$
\nabla \Phi \approx \sum_{\mathrm{k}=1}^{N_{\Omega}} \Phi^{\mathrm{k}} \nabla v^{\mathrm{k}} .
$$

The collection of equations (4.5) and (4.6) for a given i and a given $\mathrm{j}$ become

$$
\begin{gathered}
\sigma \sum_{\mathrm{k}} \Phi^{\mathrm{k}} \int_{\Omega} \nabla v^{\mathrm{k}} \cdot \nabla v^{\mathrm{i}} d V+\sum_{\mathrm{j}} I_{m}^{\mathrm{j}} \int_{\Gamma} u^{\mathrm{j}}\left[v^{\mathrm{i}}\right]_{i, e} d S=\int_{\Omega} s v^{\mathrm{i}} d V, \\
\sum_{\mathrm{k}} \Phi^{\mathrm{k}} \int_{\Gamma} u^{\mathrm{j}}\left[v^{\mathrm{k}}\right]_{i, e} d S=\sum_{1} V_{m}^{\mathrm{l}} \int_{\Gamma} u^{\mathrm{l}} u^{\mathrm{j}} d S .
\end{gathered}
$$

The left hand entries of the matrix linear system form can be defined then as

$$
A^{\mathrm{ik}}=\sigma \int_{\Omega} \nabla v^{\mathrm{k}} \cdot \nabla v^{\mathrm{i}} d V
$$

and

$$
B^{\mathrm{ij}}=\int_{\Gamma} u^{\mathrm{j}}\left[v^{\mathrm{i}}\right]_{i, e} d S .
$$

Right hand vector entries can be defined as

$$
f^{\mathrm{i}}=\int_{\Omega} s v^{\mathrm{i}} d V
$$

and

$$
G^{\mathrm{jl}}=\int_{\Gamma} u^{\mathrm{l}} u^{\mathrm{j}} d S .
$$


The collection of equations

$$
\begin{aligned}
\sum_{\mathrm{k}} \Phi^{\mathrm{k}} A^{\mathrm{ik}}+\sum_{\mathrm{j}} I_{m}^{\mathrm{l}} B^{\mathrm{ij}} & =f^{\mathrm{i}} \\
\sum_{\mathrm{k}} \Phi^{\mathrm{k}} B^{\mathrm{jk}} & =\sum_{\mathrm{l}} V_{m}^{\mathrm{l}} G^{\mathrm{jl}},
\end{aligned}
$$

can then be written in matrix form as

$$
\left[\begin{array}{cc}
\mathbf{A} & \mathbf{B} \\
\mathbf{B}^{T} & \mathbf{0}
\end{array}\right]\left[\begin{array}{c}
\mathbf{\Phi} \\
\mathbf{I}_{m}
\end{array}\right]=\left[\begin{array}{c}
\mathbf{f} \\
\mathbf{G} \mathbf{V}_{m}
\end{array}\right]
$$

with vectors $\boldsymbol{\Phi}, \mathbf{I}_{m}, \mathbf{f}$ and $\mathbf{V}_{m}$ or shortly

$$
\mathbf{M u}=\mathbf{b}
$$

with

$$
\begin{aligned}
\mathbf{M} & =\left[\begin{array}{cc}
\mathbf{A} & \mathbf{B} \\
\mathbf{B}^{T} & \mathbf{0}
\end{array}\right] \\
\mathbf{u} & =\left[\begin{array}{c}
\mathbf{\Phi} \\
\mathbf{I}_{m}
\end{array}\right] \\
\mathbf{b} & =\left[\begin{array}{c}
\mathbf{f} \\
\mathbf{G} \mathbf{V}_{m}
\end{array}\right]
\end{aligned}
$$

The matrix linear equation (4.11) has to be solved to obtain the potential $\Phi$ and membrane current $\mathbf{I}_{m}$ vectors at any time step.

\subsubsection{Reduction of the system}

Although the Laplacian $\mathbf{A}$ matrix is positive definite, in general the matrix $\mathbf{M}$ is symmetric but indefinite. In Wohlmuth and Krause [71], and in a latter improvement by Lamichhane and Wohlmuth [97], a factorization procedure was developed that can produce a reduced positive definite system after elimination of vector $\mathbf{I}_{m}$ through static condensation $^{3}$. The procedure is adapted for this work now.

\subsubsection{Obtaining of the membrane current term}

Obtaining an expression for the membrane current $\mathbf{I}_{m}$ in terms of the potential $\boldsymbol{\Phi}$ is required to reduce the system to a positive definite form and in general, to separate in two the solution process of the space/membrane problem. To achieve this a node reorganization is required. In [71] the main unknown vector ${ }^{4}$ is decomposed in three components: all the sub-domain interior nodes (i.e. nodes not touching any interface ${ }^{5}$ ),

\footnotetext{
${ }^{3}$ Differently from [71] and [97] matrices in the present work were denoted with bold letters. For instance main matrices are mapped as: $A \rightarrow \mathbf{A}, B \rightarrow \mathbf{B}$.

${ }^{4}$ In their paper this vector is known as $u_{h}$ corresponding to $\Phi$ in the present work.

${ }^{5}$ Denoted $I$ in their work
} 
the interface nodes in the mortar $\operatorname{sides}^{6}$, and the interface nodes in the non-mortar sides $^{7}$. For simplicity and because collapsing the first two does not bring any important difference, vector $\boldsymbol{\Phi}$ is now only decomposed into two components: the first containing all the nodes that do not touch any interface and the interface nodes in the "exterior" part of the interface ${ }^{8}$, and the second containing the interface nodes in the "interior" part of the interface ${ }^{9}$. The number of nodes in each part will be denoted with Roman literals c and d (not to mix up with the diameter symbol $d$ ) respectively. So $\boldsymbol{\Phi}=\left[\begin{array}{ll}\boldsymbol{\Phi}_{\mathrm{c}} & \boldsymbol{\Phi}_{\mathrm{d}}\end{array}\right]^{T}$, and the system $\mathbf{M u}=\mathbf{b}$ is decomposed as

$$
\left[\begin{array}{ccc}
\mathbf{A}_{\mathrm{cc}} & \mathbf{A}_{\mathrm{cd}} & \mathbf{L} \\
\mathbf{A}_{\mathrm{dc}} & \mathbf{A}_{\mathrm{dd}} & \mathbf{D} \\
\mathbf{L}^{T} & \mathbf{D} & \mathbf{0}
\end{array}\right]\left[\begin{array}{c}
\boldsymbol{\Phi}_{\mathrm{c}} \\
\boldsymbol{\Phi}_{\mathrm{d}} \\
\mathbf{I}_{m}
\end{array}\right]=\left[\begin{array}{c}
\mathbf{f}_{\mathrm{c}} \\
\mathbf{f}_{\mathrm{d}} \\
\mathbf{g}
\end{array}\right]
$$

with vector

$$
\mathbf{g}=\mathbf{G} \mathbf{V}_{m}
$$

and matrices

$$
\mathbf{B}=\left[\begin{array}{l}
\mathbf{L} \\
\mathbf{D}
\end{array}\right]
$$

Given the correspondence of nodes for $\boldsymbol{\Phi}_{\mathrm{d}}$ and $\mathbf{I}_{m}$ matrix $\mathbf{D}$ is diagonal [71] while matrix $\mathbf{L}$ represents the rest of elements in B. As an example, in Figure 4.1, the nodes that will correspond to $\boldsymbol{\Phi}_{\mathrm{d}}$ will be nodes 38 to 45 . The nodes associated with $\mathbf{I}_{m}$ would be nodes 1 to 8 of the addtional membrane mesh. Note that the number elements in $\mathbf{I}_{m}$ will be always $\mathrm{d}$.

Taking the second row of (4.12)

$$
\mathbf{A}_{\mathrm{dc}} \boldsymbol{\Phi}_{\mathrm{c}}+\mathbf{A}_{\mathrm{dd}} \boldsymbol{\Phi}_{\mathrm{d}}+\mathbf{D I} \mathbf{I}_{m}=\mathbf{f}_{\mathrm{d}}
$$

and rearranging, an expression for $\mathbf{I}_{m}$ can be obtained,

$$
\mathbf{I}_{m}=\mathbf{D}^{-1}\left(\mathbf{f}_{\mathrm{d}}-\mathbf{A}_{\mathrm{dc}} \boldsymbol{\Phi}_{\mathrm{d}}-\mathbf{A}_{\mathrm{dd}} \boldsymbol{\Phi}_{\mathrm{d}}\right)
$$

Introducing matrix $\mathbf{W}$ as

$$
\mathbf{W}^{T}=\left[\begin{array}{ll}
\mathbf{0} & \mathbf{D}^{-1}
\end{array}\right]
$$

the $\mathbf{I}_{m}$ term can be directly obtained from the vector difference $\mathbf{f}-\mathbf{A} \mathbf{\Phi}$ :

$$
\mathbf{I}_{m}=\mathbf{W}^{T}(\mathbf{f}-\mathbf{A} \boldsymbol{\Phi}) .
$$

The zero part of $\mathbf{W}^{T}$ erases the $\mathbf{A}_{\mathrm{cc}}$ and $\mathbf{A}_{\mathrm{cd}}$ blocks, effectively producing (4.13).

\footnotetext{
${ }^{6}$ The problem in this thesis is not exactly a mortar problem as the nodes match. The naming is just more convenient here to compare with the previous work. In their work this was denoted as $M$.

${ }^{7}$ i.e. the nodes of the interface side $\lambda_{h}$ that determine the "master" interface discretization and whose number corresponds to the number of degrees of freedom $N$.

${ }^{8}$ This corresponds to the interface nodes on the extracellular space in the biological cell case.

${ }^{9}$ This corresponds to the interface nodes on the intracellular space.
} 
In the computer implementation this decomposition can be achieved via simple node reorganization ${ }^{10,11}$.

Replacing (4.14) back into Eq. (4.11), a linear system exclusively in terms of $\boldsymbol{\Phi}$ can be obtained. The simple term $\mathbf{W}^{T}(\mathbf{f}-\mathbf{A} \boldsymbol{\Phi})$ however does not produce a symmetric positive definite system and algebraic manipulation is required to obtain the final reduced system. This is a static condensation procedure and is key to understand the last and symmetric form and for the computer implementation. Neither Wohlmuth and Krause [71], nor Lamichhane and Wohlmuth [97] presented these procedures in detail. The required operations are presented now.

\subsubsection{First static condensation}

Having $\mathbf{I}_{m}$ isolated, a first static condensation can be obtained by simply replacing $\mathbf{I}_{m}$ (4.14) in the main system (4.11):

$$
\left[\begin{array}{cc}
\mathbf{A} & \mathbf{B} \\
\mathbf{B}^{T} & \mathbf{0}
\end{array}\right]\left[\begin{array}{c}
\mathbf{\Phi} \\
\mathbf{W}^{T}(\mathbf{f}-\mathbf{A} \boldsymbol{\Phi})
\end{array}\right]=\left[\begin{array}{l}
\mathbf{f} \\
\mathbf{g}
\end{array}\right] .
$$

Extracting and manipulating the first row product

$$
\begin{aligned}
\mathbf{A} \boldsymbol{\Phi}+\mathbf{B W}^{T}(\mathbf{f}-\mathbf{A} \boldsymbol{\Phi}) & =\mathbf{f} \\
\mathbf{A} \boldsymbol{\Phi}+\mathbf{B W}^{T} \mathbf{f}-\mathbf{B W}^{T} \mathbf{A} \boldsymbol{\Phi} & = \\
\left(\mathbf{A}-\mathbf{B W}^{T} \mathbf{A}\right) \boldsymbol{\Phi} & =\left(\mathbf{I d}-\mathbf{B W}^{\mathbf{T}}\right) \mathbf{f} .
\end{aligned}
$$

Back in (4.15) the following expression is obtained

$$
\left[\begin{array}{cc}
\mathbf{A} & \mathbf{B} \\
\mathbf{B}^{T} & \mathbf{0}
\end{array}\right]\left[\begin{array}{c}
\mathbf{I d} \\
-\mathbf{W}^{T} \mathbf{A}
\end{array}\right] \boldsymbol{\Phi}=\left[\begin{array}{c}
\left(\mathbf{I d}-\mathbf{B} \mathbf{W}^{T}\right) \mathbf{f} \\
\mathbf{g}
\end{array}\right]
$$

The left hand side matrix of equation (4.16) is not square and represents an algebraic system with more equations than unknowns. Standard solution techniques such as the conjugate gradient method [101] cannot be used.

\subsubsection{Second static condensation}

An square version of the left hand side matrix can be obtained by multiplying from the left by the test functions $\left[\begin{array}{ll}\mathbf{v}^{T} & \mathbf{u}^{T}\end{array}\right]$ and restricting the space of test functions making

\footnotetext{
${ }^{10}$ In Lamichhane and Wohlmuth's latter paper [97] a transformation matrix that also reorders the elements in the vector $\mathbf{f}-\mathbf{A} \boldsymbol{\Phi}$ was introduced: matrix $E^{T}$ in their notation. This matrix was used on the computer implementation. In the next sections the notation for the semi-inverse matrix $\mathbf{W}^{T}$ is preserved.

${ }^{11}$ Two important notation changes exist between Wohlmuth and Krause's 2001 paper [71] and Lamichhane and Wohlmuth's 2004 paper [97]:

- Matrix $B$ in the 2001 paper is equivalent to matrix $B^{T}$ in the 2004 paper. For the present work the notation for the main matrix from the 2001 paper was preferred.

- In the 2001 paper matrix $W$ is equivalent to the product $\left(D^{-1} E^{T}\right)^{T}$ in the 2004 paper. For instance, in the 2001 paper $\lambda_{h}$ (which corresponds to $\mathbf{I}_{m}$ ) is defined as $\lambda_{h}=W^{T}\left(f-A u_{h}\right)$. In the 2004 paper $\lambda_{h}=D^{-1} E^{T}\left(f_{h}-A u_{h}\right)$. In the 2004 paper there is also a completely different matrix $W$ defined as $W=E D^{-1} B$.
} 
the test function $\mathbf{u}$ a function of $\mathbf{v}, \mathbf{u}=\mathbf{W}^{T} \mathbf{v}$ and

$$
\left[\begin{array}{ll}
\mathbf{v}^{T} & \mathbf{u}^{T}
\end{array}\right]\left[\begin{array}{cc}
\mathbf{A} & \mathbf{B} \\
\mathbf{B}^{T} & \mathbf{0}
\end{array}\right]\left[\begin{array}{c}
\mathbf{I d} \\
-\mathbf{W}^{T} \mathbf{A}
\end{array}\right] \mathbf{\Phi}=\left[\begin{array}{ll}
\mathbf{v}^{T} & \mathbf{u}^{T}
\end{array}\right]\left[\begin{array}{c}
\left(\mathbf{I d}-\mathbf{B W}^{T}\right) \mathbf{f} \\
\mathbf{g}
\end{array}\right] .
$$

Replacing $\mathbf{u}=\mathbf{W}^{T} \mathbf{v}$

$$
\left[\begin{array}{ll}
\mathbf{v}^{T} & \mathbf{v}^{T} \mathbf{W}
\end{array}\right]\left[\begin{array}{cc}
\mathbf{A} & \mathbf{B} \\
\mathbf{B}^{T} & \mathbf{0}
\end{array}\right]\left[\begin{array}{c}
\mathbf{I d} \\
-\mathbf{W}^{T} \mathbf{A}
\end{array}\right] \mathbf{\Phi}=\left[\begin{array}{ll}
\mathbf{v}^{T} & \mathbf{v}^{T} \mathbf{W}
\end{array}\right]\left[\begin{array}{c}
\left(\mathbf{I d}-\mathbf{B W}^{T}\right) \mathbf{f} \\
\mathbf{g}
\end{array}\right]
$$

and factorization leads to

$$
\mathbf{v}^{T}\left[\begin{array}{ll}
\mathbf{I d} & \mathbf{W}
\end{array}\right]\left[\begin{array}{cc}
\mathbf{A} & \mathbf{B} \\
\mathbf{B}^{T} & \mathbf{0}
\end{array}\right]\left[\begin{array}{c}
\mathbf{I d} \\
-\mathbf{W}^{T} \mathbf{A}
\end{array}\right] \mathbf{\Phi}=\mathbf{v}^{T}\left[\begin{array}{ll}
\mathbf{I d} & \mathbf{W}
\end{array}\right]\left[\begin{array}{c}
\left(\mathbf{I d}-\mathbf{B W}^{T}\right) \mathbf{f} \\
\mathbf{g}
\end{array}\right] .
$$

Canceling $\mathbf{v}^{T}$

$$
\left[\begin{array}{ll}
\mathbf{I d} & \mathbf{W}
\end{array}\right]\left[\begin{array}{cc}
\mathbf{A} & \mathbf{B} \\
\mathbf{B}^{T} & \mathbf{0}
\end{array}\right]\left[\begin{array}{c}
\mathbf{I d} \\
-\mathbf{W}^{T} \mathbf{A}
\end{array}\right] \boldsymbol{\Phi}=\left(\mathbf{I d}-\mathbf{B W}^{T}\right) \mathbf{f}+\mathbf{W g} .
$$

The left hand side of Eq. (4.17) is square but not symmetric. Standard techniques such as the Generalized Minimal Residual algorithm (GMRES) [102] for non-symmetric linear systems can be used.

\subsubsection{Third static condensation}

For mathematical and computational issues a symmetric system is preferred. A symmetric version of the linear system can be obtained again by restriction of the space of test functions and by algebraic manipulation of the expression for $\mathbf{I}_{m}$. By addition and subtraction of the term $\mathbf{W}^{T} \mathbf{A} \mathbf{W} \mathbf{B}^{T} \boldsymbol{\Phi}, \mathbf{I}_{m}$ in (4.14) can also be written as

$$
\mathbf{I}_{m}=\mathbf{W}^{T} \mathbf{f}-\mathbf{W}^{T} \mathbf{A} \boldsymbol{\Phi}+\mathbf{W}^{T} \mathbf{A W B}^{T} \boldsymbol{\Phi}-\mathbf{W}^{T} \mathbf{A W B}^{T} \boldsymbol{\Phi} .
$$

In the second row of (4.15) $\mathbf{B}^{T} \boldsymbol{\Phi}=\mathbf{g}$, so

$$
\begin{aligned}
\mathbf{I}_{m} & =\mathbf{W}^{T}\left(\mathbf{f}-\mathbf{A} \boldsymbol{\Phi}+\mathbf{A W B}^{T} \boldsymbol{\Phi}\right)-\mathbf{W}^{T} \mathbf{A W g} \\
& =\mathbf{W}^{T}\left(\mathbf{f}+\mathbf{A}\left(\mathbf{W B}^{T}-\mathbf{I d}\right) \boldsymbol{\Phi}\right)-\mathbf{W}^{T} \mathbf{A W g}
\end{aligned}
$$

Substituting $\mathbf{I}_{m}$ in the original system

$$
\left[\begin{array}{cc}
\mathbf{A} & \mathbf{B} \\
\mathbf{B}^{T} & 0
\end{array}\right]\left[\begin{array}{c}
\mathbf{\Phi} \\
\mathbf{W}^{T}\left(\mathbf{f}+\mathbf{A}\left(\mathbf{W B}^{T}-\mathbf{I d}\right) \boldsymbol{\Phi}\right)-\mathbf{W}^{T} \mathbf{A W g}
\end{array}\right]=\left[\begin{array}{l}
\mathbf{f} \\
\mathbf{g}
\end{array}\right]
$$

Expansion leads to

$$
\begin{aligned}
\mathbf{A} \Phi+\mathbf{B W}^{T}\left(\mathbf{f}+\mathbf{A}\left(\mathbf{W B}^{T}-\mathbf{I d}\right) \boldsymbol{\Phi}\right) & =\mathbf{f}+\mathbf{B W}^{T} \mathbf{A W g} \\
\mathbf{A} \boldsymbol{\Phi}+\mathbf{B W}^{T} \mathbf{A}\left(\mathbf{W B}^{T}-\mathbf{I d}\right) \boldsymbol{\Phi} & =\mathbf{f}+\mathbf{B W}^{T} \mathbf{A W g}-\mathbf{B W}^{T} \mathbf{f}
\end{aligned}
$$


Re-factorizing

$$
\left[\begin{array}{cc}
\mathbf{A} & \mathbf{B} \\
\mathbf{B}^{T} & \mathbf{0}
\end{array}\right]\left[\begin{array}{c}
\mathbf{\Phi} \\
\mathbf{W}^{T} \mathbf{A}\left(\mathbf{W B}^{T}-\mathbf{I d}\right) \boldsymbol{\Phi}
\end{array}\right]=\left[\begin{array}{c}
\mathbf{f}-\mathbf{B W}^{T} \mathbf{f}+\mathbf{B W}^{T} \mathbf{A W g} \\
\mathbf{g}
\end{array}\right]
$$

and extracting the common vector $\boldsymbol{\Phi}$

$$
\left[\begin{array}{cc}
\mathbf{A} & \mathbf{B} \\
\mathbf{B}^{T} & \mathbf{0}
\end{array}\right]\left[\begin{array}{c}
\mathbf{I d} \\
\mathbf{W}^{T} \mathbf{A}\left(\mathbf{W B}^{T}-\mathbf{I d}\right)
\end{array}\right] \boldsymbol{\Phi}=\left[\begin{array}{c}
\left(\mathbf{I d}-\mathbf{B W}^{T}\right) \mathbf{f}+\mathbf{B W}^{T} \mathbf{A W g} \\
\mathbf{g}
\end{array}\right] .
$$

According to [71] another possible choice for the restricted test function $\mathbf{u}$ is $\mathbf{u}=$ $\mathbf{W}^{T} \mathbf{A}\left(\mathbf{W B}^{T}-\mathbf{I d}\right) \mathbf{v}$, then $\mathbf{u}^{T}=\mathbf{v}^{T}\left(\mathbf{B W}^{T}-\mathbf{I d}\right) \mathbf{A W}$. Multiplying both sides

$$
\begin{gathered}
{\left[\begin{array}{ll}
\mathbf{v}^{T} & \mathbf{u}^{T}
\end{array}\right]\left[\begin{array}{cc}
\mathbf{A} & \mathbf{B} \\
\mathbf{B}^{T} & \mathbf{0}
\end{array}\right]\left[\begin{array}{c}
\mathbf{I d} \\
\mathbf{W}^{T} \mathbf{A}\left(\mathbf{W B}^{T}-\mathbf{I d}\right)
\end{array}\right] \mathbf{\Phi}=} \\
{\left[\begin{array}{ll}
\mathbf{v}^{T} & \mathbf{u}^{T}
\end{array}\right]\left[\begin{array}{c}
\left(\mathbf{I d}-\mathbf{B W}^{T}\right) \mathbf{f}+\mathbf{B W}^{T} \mathbf{A W g} \\
\mathbf{g}
\end{array}\right]}
\end{gathered}
$$

Expanding

$$
\begin{aligned}
& \mathbf{v}^{T}\left[\begin{array}{ll}
\mathbf{I d} & (\mathbf{B W} \\
T & -\mathbf{I d}) \mathbf{A W}
\end{array}\right]\left[\begin{array}{cc}
\mathbf{A} & \mathbf{B} \\
\mathbf{B}^{T} & \mathbf{0}
\end{array}\right]\left[\begin{array}{c}
\mathbf{I d} \\
\mathbf{W}^{T} \mathbf{A}\left(\mathbf{W B}^{T}-\mathbf{I d}\right)
\end{array}\right] \mathbf{\Phi}= \\
& \mathbf{v}^{T}\left[\begin{array}{ll}
\mathbf{I d} & (\mathbf{B W} \\
& -\mathbf{I d}) \mathbf{A W}
\end{array}\right]\left[\begin{array}{c}
\left(\mathbf{I d}-\mathbf{B W}^{T}\right) \mathbf{f}+\mathbf{B W}^{T} \mathbf{A W g} \\
\mathbf{g}
\end{array}\right]
\end{aligned}
$$

and factoring

$$
\begin{aligned}
& {\left[\begin{array}{ll}
\mathbf{I d} & \left(\mathbf{B W}^{T}-\mathbf{I d}\right) \mathbf{A W}
\end{array}\right]\left[\begin{array}{cc}
\mathbf{A} & \mathbf{B} \\
\mathbf{B}^{T} & \mathbf{0}
\end{array}\right]\left[\begin{array}{c}
\mathbf{I d} \\
\left(\left(\mathbf{B W}^{T}-\mathbf{I d}\right) \mathbf{A W}\right)^{T}
\end{array}\right] \boldsymbol{\Phi}=} \\
& \left(\mathbf{I d}-\mathbf{B W}^{T}\right) \mathbf{f}+\mathbf{B W}^{T} \mathbf{A W g}+\left(\mathbf{B W}^{T}-\mathbf{I d}\right) \mathbf{A W g}= \\
& \left(\mathbf{I d}-\mathbf{B W}^{T}\right) \mathbf{f}+\left(2 \mathbf{B W}^{T}-\mathbf{I d}\right) \mathbf{A W g} \text {. }
\end{aligned}
$$

The final system

$$
\begin{aligned}
& {\left[\begin{array}{ll}
\mathbf{I d}\left(\mathbf{B W}^{T}-\mathbf{I d}\right) \mathbf{A W}
\end{array}\right]\left[\begin{array}{cc}
\mathbf{A} & \mathbf{B} \\
\mathbf{B}^{T} & \mathbf{0}
\end{array}\right]\left[\begin{array}{c}
\mathbf{I d} \\
\left(\left(\mathbf{B W}^{T}-\mathbf{I d}\right) \mathbf{A W}\right)^{T}
\end{array}\right] \boldsymbol{\Phi}=} \\
& \left(\mathbf{I d}-\mathbf{B W}^{T}\right) \mathbf{f}+\left(2 \mathbf{B} \mathbf{W}^{T}-\mathbf{I d}\right) \mathbf{A W g}
\end{aligned}
$$

is symmetric and positive definite [71].

\subsubsection{Efficient reduction of the system}

The computer implementation of the reduction procedure does not necessarily match the mathematical version. The computer implementation of the reduction procedure and the solver should minimize the number of matrix and vector operations to speed up the solution. Detailed geometries imply that the number of elements to describe the solution domain are large and in consequence the degrees of freedom will be large. 
Particular care has been taken in the computational implementation of the algebraic operations that have to be performed for every geometry. Matrix multiplication for instance can reach $O\left(n^{3}\right)$ complexity so optimal algebraic procedures that minimize the number of matrix and vector operations are desirable. For instance, a common matrix operation such as $\mathbf{X}^{T} \mathbf{Y X}$ might have an optimized routine in the toolkit. Rearranging the system to take this form can speed up the processing. Moreover, computational libraries that handle matrix and vector operations provide restrictions on the mathematical expressions they can process, reducing verbosity and providing more efficient and compact code.

Not only processor resources but memory and can be optimized. Memory space used for intermediate matrices can be re-used lowering the total memory requirements of the program. The computer library used for the solver presented in Chapter 6 is the Portable, Extensible Toolkit for Scientific Computation: PETSc [103]. PETSc introduces other restrictions in the operations as consistency has to be maintained across several computers in parallel operations. The reorganizations in the matrix and vector operations presented next have considered in detail these restrictions and where the ones used in the final implementation of the computer solver.

\subsubsection{Efficient reduction to the symmetric system}

The symmetric condensed form in system (4.19) requires composition of an auxiliary right hand side matrix

$$
\mathbf{A}^{\prime}=\left[\begin{array}{ll}
\mathbf{I d} & \left(\mathbf{B W}^{T}-\mathbf{I d}\right) \mathbf{A W}
\end{array}\right]\left[\begin{array}{cc}
\mathbf{A} & \mathbf{B} \\
\mathbf{B}^{T} & \mathbf{0}
\end{array}\right]\left[\begin{array}{c}
\mathbf{I d} \\
\mathbf{W}^{T} \mathbf{A}\left(\mathbf{W B}^{T}-\mathbf{I d}\right)
\end{array}\right] .
$$

This can be expanded ${ }^{12}$ as a sum matrices with the size of A. Multiplying the first part

$$
\mathbf{A}^{\prime}=\left[\begin{array}{ll}
\mathbf{A}+\left(\mathbf{B W}^{T}-\mathbf{I d}\right) \mathbf{A W B}^{T} & \mathbf{B}
\end{array}\right]\left[\begin{array}{c}
\mathbf{I d} \\
\mathbf{W}^{T} \mathbf{A}\left(\mathbf{W B}^{T}-\mathbf{I d}\right)
\end{array}\right]
$$

and fully expanding

$$
\begin{aligned}
\mathbf{A}^{\prime} & =\mathbf{A}+\left(\mathbf{B} \mathbf{W}^{T}-\mathbf{I d}\right) \mathbf{A} \mathbf{W} \mathbf{B}^{T}+\mathbf{B} \mathbf{W}^{T} \mathbf{A}\left(\mathbf{W B}^{T}-\mathbf{I d}\right) \\
& =\mathbf{A}+\mathbf{B} \mathbf{W}^{T} \mathbf{A} \mathbf{W} \mathbf{B}^{T}-\mathbf{A} \mathbf{W} \mathbf{B}^{T}+\mathbf{B} \mathbf{W}^{T} \mathbf{A} \mathbf{W} \mathbf{B}^{T}-\mathbf{B W}^{T} \mathbf{A} .
\end{aligned}
$$

Factorizing

$$
\mathbf{A}^{\prime}=\mathbf{A}+2 \mathbf{B} \mathbf{W}^{T} \mathbf{A} \mathbf{W} \mathbf{B}^{T}-\mathbf{B W}^{T} \mathbf{A}-\left(\mathbf{B} \mathbf{W}^{T} \mathbf{A}\right)^{T} .
$$

The right hand side must also be treated

$$
\begin{aligned}
\mathbf{f}^{\prime} & =\left(\mathbf{I d}-\mathbf{B W}^{T}\right) \mathbf{f}+\left(2 \mathbf{B} \mathbf{W}^{T}-\mathbf{I d}\right) \mathbf{A W g} \\
& =\mathbf{f}-\mathbf{B} \mathbf{W}^{T} \mathbf{f}+\mathbf{B} \mathbf{W}^{T} \mathbf{A W g}+\mathbf{B} \mathbf{W}^{T} \mathbf{A W g}-\mathbf{A W g}
\end{aligned}
$$

\footnotetext{
${ }^{12}$ For practical reasons in the computer implementation handling of matrices with the size of $\mathbf{A}$ is simpler than building the extended and larger system $\left[\begin{array}{cc}\mathbf{A} & \mathbf{B} \\ \mathbf{B}^{T} & \mathbf{0}\end{array}\right] . \mathbf{A}^{\prime}$ has the same size than $\mathbf{A}$ and can be stored in the same memory section at the end of the system reduction. Optimized matrix/scalar routines such as $\mathbf{X}=\mathbf{X}+a \mathbf{Y}$ exist in PETSc.
} 
resulting in

$$
\mathbf{f}^{\prime}=\mathbf{f}-\mathbf{B W}^{T} \mathbf{f}+\left(2 \mathbf{B} \mathbf{W}^{T} \mathbf{A W}-\mathbf{A W}\right) \mathbf{g}
$$

In the previous expressions in can be observed that the term $\mathbf{B W}^{T}$ repeats several times. In the computed implementation this matrix can even be directly assembled from the finite elements without the matrix product. From decomposition (4.12) notice that $\mathbf{B}=\left[\begin{array}{ll}\mathbf{L} & \mathbf{D}\end{array}\right]^{T}$ and $\mathbf{W}^{T}=\left[\begin{array}{ll}\mathbf{0}_{d c} & \mathbf{D}^{-1}\end{array}\right]$, this implies

$$
\mathbf{B W}^{T}=\left[\begin{array}{cc}
\mathbf{0}_{c c} & \left(\mathbf{L D}^{-1}\right)_{c d} \\
\mathbf{0}_{d c} & \mathbf{I d}_{d d}
\end{array}\right]
$$

The product $\mathbf{L} \mathbf{D}^{-1}$ is a matrix were each row corresponds to the one of the nodes that do not touch any membrane or the interface nodes in the exterior part of the membrane. Each column corresponds to one of the nodes in the interior membrane. The matrix itself consists of 0 or -1 values. Values of -1 appear where an exterior interface node matches an interior interface node. Regardless of the value of the entries in $\mathbf{B}$ this can be seen with a simple example for a system with four interface nodes with test function coefficients $b_{\mathrm{i}}$ :

$$
\mathbf{B}=\left[\begin{array}{c}
\mathbf{L} \\
\mathbf{D}
\end{array}\right]=\left[\begin{array}{cccc} 
& -b_{1} & & \\
& & -b_{2} & \\
\vdots & & & \\
-b_{0} & & & \\
\vdots & & & \\
b_{0} & & & -b_{3} \\
& b_{1} & & \\
& & b_{2} & \\
& & & b_{3}
\end{array}\right]
$$

This implies

$$
\mathbf{L D}^{-1}=\left[\begin{array}{cccc} 
& -b_{1} & & \\
& & -b_{2} & \\
\vdots & & & \\
-b_{0} & & & \\
\vdots & & & \\
& & & -b_{3}
\end{array}\right]\left[\begin{array}{cccc}
1 / b_{0} & & & \\
& 1 / b_{1} & & \\
& & 1 / b_{2} & \\
& & & 1 / b_{3}
\end{array}\right]
$$

so

$$
\mathbf{L D}^{-1}=\left[\begin{array}{cccc} 
& -1 & & \\
& & -1 & \\
\vdots & & & \\
-1 & & \\
\vdots & & & \\
& & & -1
\end{array}\right]
$$


Matrix $\mathbf{B W}^{T}$ can be easily constructed in the assembly procedure. The new matrix will be defined as

$$
\mathbf{N}=-\mathbf{B W}^{T}
$$

With the negative sign to save one scalar-matrix operation in the matrices toolkit.

The term $-\mathbf{B W}^{T} \mathbf{A}$ is also common in (4.20) and (4.21). This matrix will be called P:

$$
\mathbf{P}=\mathbf{N A}=-\mathbf{B W}^{T} \mathbf{A} .
$$

Now, using (4.20) and (4.21) the system (4.19) is

$$
\mathbf{A}^{\prime} \Phi=\mathbf{f}^{\prime}
$$

So

$$
\left(\mathbf{A}+\mathbf{P}+\mathbf{P}^{T}+2 \mathbf{P} \mathbf{N}^{T}\right) \mathbf{\Phi}=\mathbf{f}+\mathbf{N f}+(-2 \mathbf{P W}-\mathbf{A W}) \mathbf{g}
$$

Producing and storing the transpose of $\mathbf{N}$ can be avoided. Matrix $\mathbf{P} \mathbf{N}^{T}$ is symmetric so

$$
\mathbf{P N}^{T}=\left(\mathbf{P} \mathbf{N}^{T}\right)^{T}=\mathbf{N P}^{T} .
$$

At the same time, the transpose $\mathbf{P}^{T}$ which needs to be computed, can be used twice. Two additional auxiliary matrices can also be defined:

$$
\begin{gathered}
\mathbf{Q}=\mathbf{A W} \\
\mathbf{R}=-2 \mathbf{P W}-\mathbf{Q}
\end{gathered}
$$

Matrix $\mathbf{R}$ only has to be computed once for the right hand side. Finally,

$$
\left[\mathbf{A}+\mathbf{P}+\mathbf{P}^{T}+2\left(\mathbf{N P}^{T}\right)\right] \mathbf{\Phi}=\mathbf{f}+\mathbf{N f}+\mathbf{R g}
$$

The $\mathbf{I}_{m}$ term could be obtained from the long expression (4.18) but also from the shorter Eq. (4.14):

$$
\mathbf{I}_{m}=\mathbf{W}^{T}(\mathbf{f}-\mathbf{A} \boldsymbol{\Phi})=\mathbf{W}^{T} \mathbf{f}-\mathbf{W}^{T} \mathbf{A} \boldsymbol{\Phi}
$$

so the transpose of matrix $\mathbf{Q}$ can be reused when $\mathbf{I}_{m}$ is calculated as

$$
\mathbf{I}_{m}=\mathbf{W}^{T} \mathbf{f}-\mathbf{Q}^{T} \boldsymbol{\Phi} .
$$

In the above operations three specific PETSc aspects were considered:

- PETSc can do fast multiplications with products of the form $\mathbf{X}^{T} \mathbf{A X}$. However doing $2 \mathbf{N P}^{T}$ is better than $2 \mathbf{N A} \mathbf{N}^{T}$ because: in both cases a new matrix has to be created; the transpose of $N$ is not required anywhere else; and the product NA is already in $\mathbf{P}$.

- In cases as the right hand side product $(-2 \mathbf{P W}-\mathbf{Q}) \mathbf{g}$ is preferred to have a single matrix to multiply with as this has to be done every timestep ( $\mathrm{g}$ is not constant). That is why matrix $\mathbf{R}$ is created.

- At the end of every timestep $\mathbf{I}_{m}$ has to be calculated so a copy of $\mathbf{W}^{T}$ is needed in any case. It would be expensive to multiply $\mathbf{W}^{T}$ and $\mathbf{A}$ every time step so matrix $\mathbf{Q}$ was created. $\mathbf{Q}$ could also use less memory space than $\mathbf{A}$.

With this procedure only four matrix products, four matrix sums and four vector sums are required in total. 


\subsubsection{Efficient reduction to the asymmetric system}

The asymmetric system (4.17) will be used in a specific solution scheme with the CrankNicolson solver. Expanded

$$
\begin{aligned}
{\left[\begin{array}{cc}
\mathbf{A}+\mathbf{W B}^{T} & \mathbf{B}
\end{array}\right]\left[\begin{array}{c}
\mathbf{I d} \\
-\mathbf{W}^{T} \mathbf{A}
\end{array}\right] \mathbf{\Phi} } & =\mathbf{f}-\mathbf{B W}^{T} \mathbf{f}+\mathbf{W g} \\
\left(\mathbf{A}+\mathbf{W B}^{T}-\mathbf{B} \mathbf{W}^{T} \mathbf{A}\right) \boldsymbol{\Phi} & =
\end{aligned}
$$

For the computer implementation ${ }^{13}$ matrix $\mathbf{N}$ can again be used

$$
\mathbf{N}=-\mathbf{B W}^{T}
$$

Matrix $\mathbf{G}$ in $\mathbf{g}=\mathbf{G V}_{m}$ from (4.11) needs to be preserved so an auxiliary matrix $\mathbf{S}$ is created

$$
\mathbf{S}^{T}=\mathbf{N G}
$$

The asymmetric system is then

$$
\left(\mathbf{A}-\mathbf{N}^{T}+\mathbf{N A}\right) \boldsymbol{\Phi}=\mathbf{f}+\mathbf{N f}+\mathbf{S}^{T} \mathbf{V}_{m}
$$

The membrane current is calculated with auxiliary matrix ${ }^{14} \mathbf{T}$

$$
\mathbf{T}=-W^{T} A
$$

so

$$
\mathbf{I}_{m}=\mathbf{W}^{T} \mathbf{f}+\mathbf{T} \Phi
$$

\subsection{Time discretization}

Equation (2.41) is integrated at time step $\mathrm{n}$ after solution of the potential equations (2.54) and (2.55), preserving the continuity condition (2.53). Each step the potential $\boldsymbol{\Phi}^{\mathrm{n}}$ is obtained for a fixed voltage vector $\mathbf{V}_{m}^{\mathrm{n}}$ using the linear system from Section 4.4. Two alternative time integration schemes can then be applied and are now presented.

\subsubsection{Forward Euler}

After finding $\boldsymbol{\Phi}^{\mathrm{n}}$, the simplistic explicit Euler method can be used to produce membrane current $\mathbf{I}_{m}^{\mathrm{n}}\left(\boldsymbol{\Phi}^{\mathrm{n}}\right)$ using (4.14). This last, together with the vector of ionic currents per node $\mathbf{I}_{i o n}^{\mathrm{n}}\left(\mathbf{V}_{m}^{\mathrm{n}}\right)$, is used to find the new membrane voltage $\mathbf{V}^{\mathrm{n}+1}$. The discrete form of the explicit time equation can be written as

$$
\mathbf{V}_{m}^{\mathrm{n}+1}=\mathbf{V}_{m}^{\mathrm{n}}+\frac{\Delta t}{C_{m}}\left(\mathbf{I}_{m}^{\mathrm{n}}\left(\Phi^{\mathrm{n}}\left(\mathbf{V}_{m}^{\mathrm{n}}\right)\right)-\mathbf{I}_{i o n}^{\mathrm{n}}\left(\mathbf{V}_{m}^{\mathrm{n}}, \mathbf{q}_{i o n}^{n+1}\right)\right)
$$

\footnotetext{
${ }^{13}$ For historical reasons in the current computer implementation matrix $\mathbf{N}$ corresponds to matrix $-\mathbf{X}$.

${ }^{14}$ Matrix $\mathbf{T}$ is called matrix $\mathbf{R}$ in the code.
} 
State variables for the ionic currents can also be approximated explicitly as

$$
\mathbf{q}_{i o n}^{\mathrm{n}+1}=\mathbf{q}^{\mathrm{n}}+\Delta t \mathbf{f}\left(\mathbf{V}_{m}^{\mathrm{n}}, \mathbf{q}_{i o n}^{\mathrm{n}}\right) .
$$

The new $\mathbf{V}_{m}^{n+1}$ is then ready to be used again to solve the spatial equations. The spatial equation for the explicit Euler can be solved with the optimized reduced system (4.22). The Euler scheme is limited and requires extremely small time steps. Chapter 5 will be dedicated to explain this restriction.

\subsubsection{Implicit Crank-Nicolson}

The Crank-Nicolson is an implicit, backward difference method in time, developed for the numerical solution of the parabolic diffusion equation [55]. For the diffusion equation with finite difference methods the Crank-Nicolson method is unconditionally stable, meaning that regardless of the time step size the numerical solution always converges [104]. As a backward method, the method involves an unknown, $n+1$ term in the right hand side of the time step equation. In the finite difference scheme for the diffusion equation this unknown is treated by a simple algebraic manipulation displacing the linear term to the left side and employing the linear solver to find a solution [105]. Due to the membrane discontinuity, the same artifice requires a special treatment in the present case.

To integrate the time equation (2.41) the Crank-Nicolson requires the value of the membrane current at step $n+1$ in:

$$
\mathbf{V}_{m}^{\mathrm{n}+1}=\mathbf{V}_{m}^{\mathrm{n}}+\frac{\Delta t}{C_{m}}\left(\frac{1}{2}\left(\mathbf{I}_{m}^{\mathrm{n}+1}\left(\boldsymbol{\Phi}^{\mathrm{n}+1}\right)+\mathbf{I}_{m}^{\mathrm{n}}\left(\boldsymbol{\Phi}^{\mathrm{n}}\right)\right)-\mathbf{I}_{i o n}^{\mathrm{n}}\left(\mathbf{V}_{m}^{\mathrm{n}}, \mathbf{q}_{i o n}^{n+1}\right)\right) .
$$

$\mathbf{I}_{m}^{\mathrm{n}+1}$ is the unknown future value of $\mathbf{I}_{m}$.

Through term re-arrangement the spatial linear solver can be used. Defining $\mathbf{h}^{\mathrm{n}}=$ $\mathbf{V}_{m}^{\mathrm{n}}+\frac{\Delta t}{C_{m}}\left(\frac{1}{2} \mathbf{I}_{m}^{\mathrm{n}}-\mathbf{I}_{i o n}^{\mathrm{n}}\right)$ one gets

$$
\begin{aligned}
\mathbf{V}_{m}^{\mathrm{n}+1} & =\frac{\Delta t}{2 C_{m}} \mathbf{I}_{m}^{\mathrm{n}+1}+\mathbf{V}_{m}^{\mathrm{n}}+\frac{\Delta t}{C_{m}}\left(\frac{1}{2} \mathbf{I}_{m}^{\mathrm{n}}-\mathbf{I}_{i o n}^{\mathrm{n}}\right) \\
& =\frac{\Delta t}{2 C_{m}} \mathbf{I}_{m}^{\mathrm{n}+1}+\mathbf{h}^{\mathrm{n}} .
\end{aligned}
$$

Combining $\mathbf{V}_{m}^{\mathrm{n}+1}$ with the linear system (4.11) in the advanced time step $\mathrm{n}+1$ results in

$$
\left[\begin{array}{cc}
\mathbf{A} & \mathbf{B} \\
\mathbf{B}^{T} & \mathbf{0}
\end{array}\right]\left[\begin{array}{c}
\mathbf{\Phi}^{\mathrm{n}+1} \\
\mathbf{I}_{m}^{\mathrm{n}+1}
\end{array}\right]=\left[\begin{array}{c}
\mathbf{f}^{\mathrm{n}} \\
\mathbf{G}\left(\frac{\Delta t}{2 C_{m}} \mathbf{I}_{m}^{\mathrm{n}+1}+\mathbf{h}^{\mathrm{n}}\right)
\end{array}\right]
$$

The lower row produces

$$
\mathbf{B}^{T} \boldsymbol{\Phi}^{\mathrm{n}+1}=\frac{\Delta t}{2 C_{m}} \mathbf{G I}_{m}^{\mathrm{n}+1}+\mathbf{G h}_{v}^{\mathrm{n}}
$$

which reordered is

$$
\mathbf{B}^{T} \boldsymbol{\Phi}^{\mathrm{n}+1}-\frac{\Delta t}{2 C_{m}} \mathbf{G I}_{m}^{\mathrm{n}+1}=\mathbf{G h}_{v}^{\mathrm{n}} .
$$


With $\mathbf{C}=-\frac{\Delta t}{2 C_{m}} \mathbf{G}$ the Crank-Nicolson linear system ${ }^{15}$ is

$$
\left[\begin{array}{cc}
\mathbf{A} & \mathbf{B} \\
\mathbf{B}^{T} & \mathbf{C}
\end{array}\right]\left[\begin{array}{c}
\boldsymbol{\Phi}^{\mathrm{n}+1} \\
\mathbf{I}_{m}^{\mathrm{n}+1}
\end{array}\right]=\left[\begin{array}{c}
\mathbf{f}^{\mathrm{n}} \\
\mathbf{G h}^{\mathrm{n}}
\end{array}\right]
$$

In this form the system could be solved with the GMRES algorithm [102] but a system in terms of $\boldsymbol{\Phi}$ only is easier to handle. Unfortunately the system (4.26) is different from system (4.11) and it cannot be solved with the optimized reduced system (4.22).

As $\mathbf{C} \neq \mathbf{0}$ a simple solution could be obtained using the Schur complement [106]. The last row of (4.26) produces

$$
\mathbf{B}^{T} \boldsymbol{\Phi}^{\mathrm{n}+1}+\mathbf{C I}_{m}^{\mathrm{n}+1}=\mathbf{G h}^{\mathrm{n}}
$$

and reordering and inverting $\mathbf{C}$

$$
\mathbf{I}_{m}^{\mathrm{n}+1}=\mathbf{C}^{-1}\left(\mathbf{G g}_{v}^{\mathrm{n}}-\mathbf{B}^{T} \boldsymbol{\Phi}^{\mathrm{n}+1}\right) .
$$

In the first system row

$$
\mathbf{A} \Phi^{\mathrm{n}+1}+\mathbf{B I}_{m}^{\mathrm{n}+1}=\mathbf{f}^{\mathrm{n}}
$$

$\mathbf{I}_{m}^{\mathrm{n}+1}$ can be replaced

$$
\begin{aligned}
\mathbf{A} \Phi^{\mathrm{n}+1}+\mathbf{B C}^{-1}\left(\mathbf{G g}_{v}^{\mathrm{n}}-\mathbf{B}^{T} \boldsymbol{\Phi}^{\mathrm{n}+1}\right) & =\mathbf{f}^{\mathrm{n}} \\
\mathbf{A} \boldsymbol{\Phi}^{\mathrm{n}+1}-\mathbf{B C}^{-1} \mathbf{B}^{T} \boldsymbol{\Phi}^{\mathrm{n}+1} & =\mathbf{f}^{\mathrm{n}}-\mathbf{B} \mathbf{C}^{-1} \mathbf{G h}_{v}^{\mathrm{n}} .
\end{aligned}
$$

The new reduced system is

$$
\left(\mathbf{A}-\mathbf{B C}^{-1} \mathbf{B}^{T}\right) \boldsymbol{\Phi}^{\mathrm{n}+1}=\mathbf{f}^{\mathrm{n}}-\mathbf{B} \mathbf{C}^{-1} \mathbf{G h}_{v}^{\mathrm{n}} .
$$

The membrane current could be found as

$$
\mathbf{I}_{m}^{\mathrm{n}+1}=-\frac{1}{h} \mathbf{h}_{v}^{\mathrm{n}}-\mathbf{C}^{-1} \mathbf{B}^{T} \boldsymbol{\Phi}^{\mathrm{n}+1} .
$$

The Schur complement solution is quite easy to implement but requires inversion of matrix $\mathbf{C}$. Although $\mathbf{C}$ is sparse, in general the inverse of a sparse matrix is not, making this approach memory inefficient.

Presence of matrix $\mathbf{C}$ makes the static condensation of Section 4.4.3.4 asymmetric. Lacking the theoretical background to find a symmetric version of this matrix, the procedure for obtaining the asymmetric version of the system (4.23) was used. For numerical solution the GMRES algorithm [102] was then employed.

Contrary to the Euler method, any solution to (4.26) depends on the previous $\mathbf{I}_{m}$ (implicit in $\mathbf{h}^{\mathrm{n}}$ ). This makes the method particularly weak at initialization and fast changing exterior stimuli. A better solution can be obtained by pre-calculating an intermediate step $\mathbf{I}_{m}^{\mathrm{n}+\frac{1}{2}}$ in a predictor-corrector scheme. This improvement is referred as the Euler-Crank-Nicolson (ECN) method and a computer implementation is shown in the appendix Section G.3.

\footnotetext{
${ }^{15}$ Although $c$ is writen as constant $\Delta t$ might be variable in time (e.g. in adaptive time steps) and $C_{m}$ variable in space (e.g. the axon myelin sheet). In such cases $1 / C_{m}$ should be a matrix and the left hand side would need to be scaled properly each time step. The $1 / C_{m}$ matrix is implemented in the current version of the computer solver.
} 


\subsection{Cable solvers}

A specific type of solvers for the space/membrane equations are computational solvers of the cable equation. These can simultaneously solve the membrane voltage for treeshaped structures made of simpler cables. Cable equation solvers can model combinations of non-linear ionic currents representing neurons in close to realistic set-ups. Besides the state variables required for ionic currents, cable solvers deal mainly with a single variable i.e. the membrane voltage along the cable. By comparison, a full three-dimensional solver like the one proposed in this work has to deal with two main unknowns: potential in space (which directly determines the membrane voltage) and membrane current (calculated on the membrane surface). Although in principle different, it is interesting to review cable solvers.

Several free accessible solvers of the cable equation exist, the most notable are NEURON [12] and GENESIS (GEneral NEural SImulation System) [107]. Other less known alternatives include Surf-Hippo [108] and NeuroDUNE [109]. With the exception of NeuroDUNE, which uses a one dimensional finite volume method, all these packages use finite differences to solve the spatial part of the cable equation. In the finite differences method, a cable equation (2.50) for a piece of cable $\mathrm{j}$ of length $\Delta x$, voltage $V_{m}^{\mathrm{j}}$ and adjacent, identical cable section potentials $V_{m}^{\mathrm{j}+1}, V_{m}^{\mathrm{j}-1}$ reads [12]:

$$
\frac{d}{4 R_{i}} \frac{V_{m}^{\mathrm{j}+1}-2 V_{m}^{\mathrm{j}}+V_{m}^{\mathrm{j}-1}}{\Delta x^{2}}=C_{m} \frac{\partial V_{m}^{\mathrm{j}}}{\partial t}+I_{i o n}^{\mathrm{j}}
$$

An implicit in time, finite difference version of this equation reads

$$
\begin{aligned}
C_{m} \frac{\left(V_{m}^{\mathrm{j}}(t+\Delta t)-V_{m}^{\mathrm{j}}(t)\right)}{\Delta t}= & \frac{d}{4 R_{i}} \frac{V_{m}^{\mathrm{j}+1}(t+\Delta t)-2 V_{m}^{\mathrm{j}}(t+\Delta t)+V_{m}^{\mathrm{j}-1}(t+\Delta t)}{\Delta x^{2}} \\
& -I_{i o n}^{\mathrm{j}}(t+\Delta t) .
\end{aligned}
$$

Obtaining a linear algebraic system for this equation is relatively simple. Voltage terms in the new step $t+\Delta t$ are all linear and can be set in the left hand side of the equation. In combination with all other cable sections, a system of equations can be formed with the adjacent terms of $V_{m}$. The final algebraic system matrix will have entries only for the connected sections.

Other properties of the neuron structure facilitate the numerical solution of the cable equation. The four computer solvers presented above employ the core numerical algorithms proposed by Hines [110, 111, 112]. The so called "Hines method" consists of three strategies:

- Take advantage of the tree structure of neurons to construct a linear system for the spatial variables whose main matrix is almost tridiagonal (non diagonal only on branching points). Being nearly tridiagonal, the linear system can be solved fast with Gaussian elimination. An $O(n)$ Gaussian elimination algorithm can be written taking advantage of this structure. The speed up for trees is comparable to what would be achievable for a single unbranched cable. 
- Hodgkin-Huxley like membrane currents can all be written in the form

$$
I_{x}=g_{x}\left(V_{m}-E_{x}\right)
$$

with $x$ any of the channel types. Although $g_{x}$ involves non linear terms, if its value is known, the current density $I_{x}$ can be written linearly with respect to $V_{m}$. The second Hines strategy proposes calculating $g_{x}$ at an intermediate step $t+\frac{\Delta t}{2}$ and keeping $V_{m}$ for the next time step preserving accuracy, that is

$$
I_{x}(t+\Delta t)=g_{x}\left(V_{m}(t), g_{x}\left(t-\frac{\Delta t}{2}\right)\right)\left(V_{m}(t+\Delta t)-E_{x}\right) .
$$

In this form, the currents can be added in the calculation of the step $t+\Delta t$ as linear terms of $V_{m}(t+\Delta t)$ and implicit methods used.

- The last strategy proposes the use of pre-multiplied Hodgkin-Huxley rate function tables. The conductance variables in Hodgkin-Huxley type models are independent from each other and all have exponential function dependencies in $V_{m}$. The term $V_{m}$ remains within a relatively short range for biologically realistic values (e.g. - 100 to $100 \mathrm{mV}$ ). Every conductance variable can then be integrated in the form

$$
q\left(t+\frac{\Delta t}{2}\right)=P\left(\alpha_{q}, \beta_{q}\right)+Q\left(\alpha_{q}, \beta_{q}\right) q\left(t-\frac{\Delta t}{2}\right)
$$

with $P, Q$ some function of $\alpha_{q}$ and $\beta_{q}$ which are in turn functions of $V_{m}$ only. Static tables for $P$ and $Q$ as a function of $V_{m}$ can be created and interpolated to obtain continuous values for $V_{m}$ in biophysically relevant ranges.

Hines reported a 10 to 20 -fold reduction in the amount of time required to solve the cable equation compared to more "brute force" explicit methods. Note that these experiments were performed in the 80's and modern computers do not benefit so much from the third strategy.

An implicit scheme such as the Crank-Nicolson method has been used in NEURON and can be configured in GENESIS [112]. Although it achieves better accuracy compared to a backward Euler method, the latter is preferred as it does not produce oscillations in the solution for large timesteps [12].

Besides the use of static rate function tables, no other of these considerations were taken during the development of the space/membrane equations tool. The first Hines strategy could not be used as the full tree-dimensional structure that defines the membrane and extracellular space is far more dense. The second Hines strategy could be used in future versions of the tool.

It should be noted that the NEURON simulation environment allows for a simplified representation of the extracellular space. This is added as an exterior core conductor but the flux of currents is only allowed along the cable making it essentially one-dimensional.

\subsection{Summary}

Solution of the space/membrane equations for general three-dimensional geometries requires numerical methods. The main method of solution proposed by this work was 
presented in this chapter. Although previous approaches have tried to provide solutions for the space/membrane equations they were limited by two main aspects: computational restrictions to represent detailed geometries and the lack of efficient time evolution schemes. From the partial differential equation solvers available, the finite element method was preferred. Availability of other similar tools that could be reused was one of the main reasons for this choice as will be discussed in Chapter (6).

The core of the solution strategy lies in the separation of the spatial problem and the time evolution. This separation is supported by the quasi-static approximation and facilitates the development of computer algorithms for the solution. For the spatial solution a static condensation procedure based on the static equations of Wohlmuth and coworkers was used and a optimized computer version of it was developed.

Time integration schemes for the forward Euler and the Crank-Nicolson method were presented. The use of the Crank-Nicolson scheme is innovative and has not been found in the literature for equivalent problems. Optimized methods, that can help speed up the matrix operations in the computer were presented. The details presented in this chapter are fundamental for the software implementation of the finite element tool. The one-dimensional, tree-like structure dependence in a single variable, and many other simplifications make the solution of the cable equation ideal for obtaining of fast results in neuron simulation. Lessons can be learned from cable solvers. 


\section{Stability of the numerical method}

\subsection{Introduction}

The numerical treatment of any set of partial differential equations needs to be carefully scrutinized. In the previous chapter, a procedure to bring the space/membrane equations to a discrete form was shown. However, derivation of the discrete equations alone does not imply a correct solution. Inadequate numerical strategies can affect the accuracy of the result and even, completely miss the solution. To ensure that a solution obtained by a numerical scheme is true and accurate, validation of the scheme is required. A valid partial differential equation solution scheme must be stable, consistent and convergent. Stability and consistency are in general easy to prove, while convergence normally requires demanding functional analysis. Fortunately, it is enough to show stability and consistency as convergence follows from the Lax-Richtmyer equivalence theorem [105].

In this chapter the numerical strategy presented in Chapter 4 is validated through stability analysis. On the literature, the forward Euler, Crank-Nicolson, and finite element method are well known to be consistent and this will not be shown. An approximation to the stability criterion for the scheme will be obtained first from the recurrence relation, and then with a von Neumann analysis. The non standard form of the time integration expressions (4.24) and (4.25) required particular assumptions but these are justified during this chapter and corroborated with numerical experiments. In the next sections the rationale of the proof is given first and the mathematical procedure follows. The final sections discuss the results and summarize the chapter. In Chapter 7 convergence is shown numerically.

The theory behind stability analysis is not simple and is left to the reader. Informative and mathematically accessible resources to this theory can be found in the lecture notes of Trefethen [105], Olver [94] and Otto [113] and in the book of Strikwerda [114]. Stability analysis has also been performed before for cable solvers [115].

\subsection{Rationale}

Implementation of the discrete version of the space/membrane equations preceded the mathematical stability analysis. Once the computer version of the solver was functional, numerical experiments followed in order to validate the approach. The first choice for a time iteration scheme was the simpler forward Euler method but the results were not acceptable. The very simple simulation of a two dimensional cell under an extra- 
cellular field required time steps of nanoseconds to converge (Figure 5.1). The goal of the tool was to perform simulations of tens to hundred of milliseconds and these restrictions proved inadequate. With little fundamental background, implementation of the Crank-Nicolson scheme came after and the empirical results showed an impressive improvement. With Crank-Nicolson, time steps of hundreds of microseconds were possible.

Two questions emerged then: First, what elements of the system determined the numerical divergence in the Euler case?, and second, how robust would be the CrankNicolson for other parameters?. A numerical experiment was devised to better understand the relation between the time step $\Delta t$ and the typical spatial length parameter $h$. The question was how, as $h$ was reduced, $\Delta t$ had to be reduced to keep the solution stable. The experiment consisted on simulating a circular and a spherical cell under a field with the forward Euler method (Figure 5.1). The tests were performed for different cell diameters (to see if $d$ had any influence) and decreasing values of $h$. The results for these experiments are shown in Figure 5.2. Fits of the values showed an $h^{a}$ relation with $a$ between 0.7 and 1.9. The results also seemed to show a dimensionality dependence from the $2 \mathrm{D}$ and $3 \mathrm{D}$ case.

An attempt to find the $\Delta t$ and $h$ relation analytically was tried then. Full analysis of the space discretization restrictions for the space/membrane equations was however not simple. The piecewise nature of the problem, together with the separation of the time and space solutions were at the center of the problem. Algebraically this implied that the Forward Euler 4.24 and Crank-Nicolson 4.25 expressions needed to be written in terms of $V_{m}$ and not as expressions of $V_{m}$ and $I_{m}$. A similar analysis was not found in the literature or in textbook examples, which only consider spatially continuous problems such as in the diffusion equation.

The first approach to understand this relation was an approximation through the space independent recurrence relation $V_{m}^{\mathrm{n}+1}=\alpha V_{m}^{\mathrm{n}}+\beta$ (Section 5.3) for the pair of infinite membranes (Section 3.3). This analysis indicated that the cell time constant (Section 3.4) was dominant in the forward Euler solution. The cell time constant is in the order of nanoseconds explaining why the Euler method required such small time steps. Surprisingly the same analysis revealed that the Crank-Nicolson method was stable for time steps up to the membrane time constant. The membrane time constant is usually larger than 1 millisecond showing for the first time the robustness of the method.

Still the stability picture was not complete as the $h$ parameter could not be included in the first analysis. One more approximation combined the recurrence relation approach with a simplified space discretization and showed similar but partial results. This is shown in Sections 5.4 and 5.5. Stability analysis tools [114] from finite difference methods were used at last. This approach was still not free from the $V_{m}, I_{m}$ issue and the previous approaches had to be combined with von Neumann analysis [104] for a final approximation. This is shown in 5.6. An alternative to von Neumann analysis, the "matrix method" [114] required an analytical expression for the full FEM matrix and its inverse and this was not attempted due to its complexity.

In the next sections the progression of approximations to the stability analysis is 

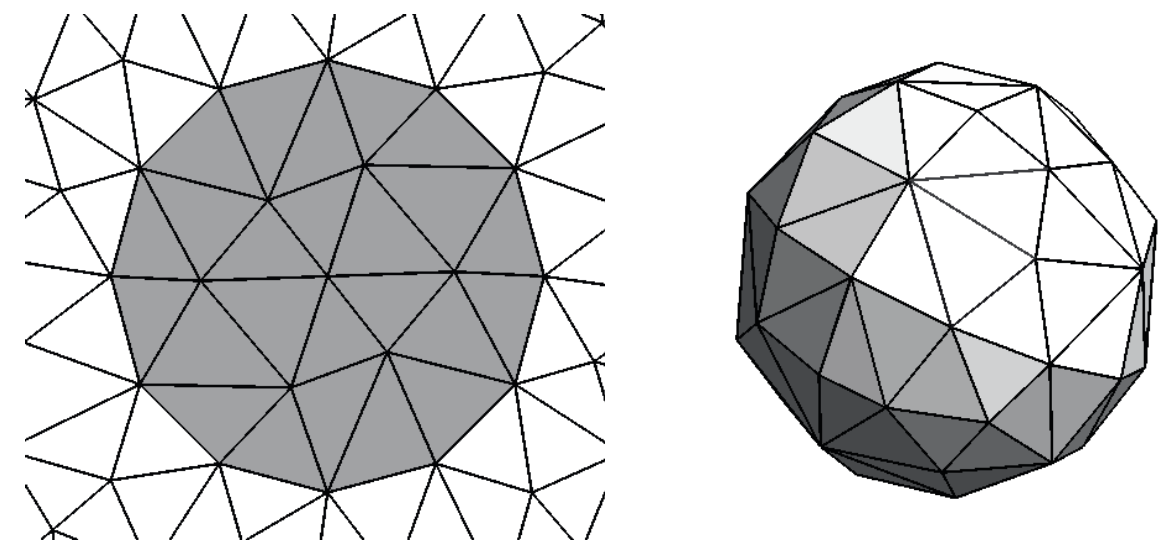

Figure 5.1: Examples of the Circular and spherical cells of changing diameter $(d)$ and typical length $(h)$ parameter. In this case $d=20 \mu \mathrm{m}$ and $h=8 \mu \mathrm{m}$. The cells were put under a $10^{4} \mathrm{mV} / \mathrm{cm}$ constant homogeneous stimulus and simulated with forward Euler.
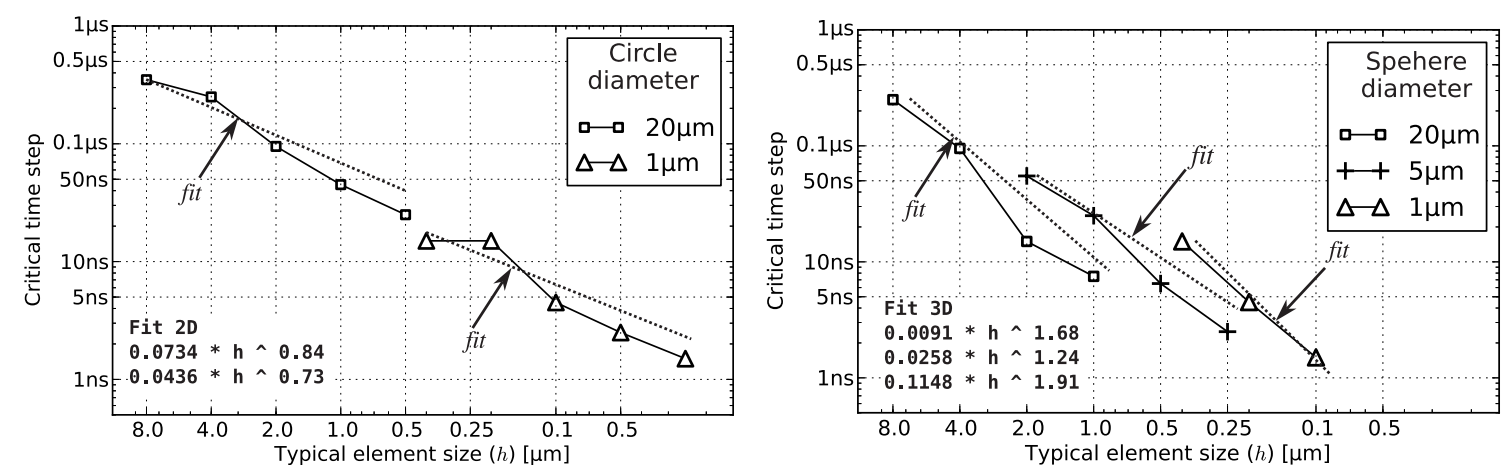

Figure 5.2: Experimental results finding the critical time step for a circular and spherical cell. The solution was stable above the solid lines. Dotted lines represent the fits for the $\Delta t$ and $h$ relation. The criterion for stability was that the simulation remained stable in steady state at least up to $t=10 \tau_{c}$.

shown. Due to the non standard form of the equations, a direct approximation with the von Neumann stability would not have been simple. The following steps are considered introductory to it.

\subsection{Analysis with the space independent recurrence relation}

A first approximation to the stability criterion can be obtained for the simple pair of parallel infinite membranes (Section 3.3). The discrete time forward Euler scheme (4.24) for the infinite membranes (3.2) is

$$
V_{m}^{\mathrm{n}+1}=\left(1-\frac{2 \sigma \Delta t}{C_{m} D}-\frac{\Delta t}{C_{m} R_{m}}\right) V_{m}^{\mathrm{n}}+\frac{I_{E} \Delta t}{C_{m}} .
$$

This is a recurrence relation [116]. 
With $\tau_{m}=C_{m} R_{m}$ and rearranging the terms,

$$
\begin{aligned}
V_{m}^{\mathrm{n}+1} & =V_{m}^{\mathrm{n}}+\frac{\Delta t}{C_{m}}\left(I_{E}-\frac{V_{m}^{\mathrm{n}}}{D / 2 \sigma}-\frac{V_{m}^{\mathrm{n}}}{R_{m}}\right) \\
& =V_{m}^{\mathrm{n}}+\frac{\Delta t}{C_{m}} I_{E}-\frac{\Delta t}{C_{m} D / 2 \sigma} V_{m}^{\mathrm{n}}-\frac{\Delta t}{\tau_{m}} V_{m}^{\mathrm{n}} \\
& =\left(1-\frac{\Delta t}{C_{m} D / 2 \sigma}-\frac{\Delta t}{\tau_{m}}\right) V_{m}^{\mathrm{n}}+\frac{\Delta t}{C_{m}} I_{E} .
\end{aligned}
$$

Defining $\alpha_{0}=1-\frac{\Delta t}{C_{m} D / 2 \sigma}-\frac{\Delta t}{\tau_{m}}$ and $\beta_{0}=\frac{\Delta t}{C_{m}} I_{E}$, the recurrence relation can be re-written as:

$$
V_{m}^{\mathrm{n}+1}=\alpha_{0} V_{m}^{\mathrm{n}}+\beta_{0} .
$$

Assuming the initial voltage of the cell is zero $V_{m}^{0}=0$, this expands to the series:

$$
\begin{aligned}
V_{m}^{1} & =\alpha_{0} V_{m}^{0}+\beta_{0}=\beta_{0} \\
V_{m}^{2} & =\alpha_{0} \beta_{0}+\beta_{0} \\
V_{m}^{3} & =\alpha_{0}\left(\alpha_{0} \beta_{0}+\beta_{0}\right)+\beta_{0}=\alpha_{0}^{2} \beta_{0}+\alpha_{0} \beta_{0}+\beta_{0} \\
V_{m}^{4} & = \\
& \vdots \\
V_{m}^{\mathrm{n}} & =\sum_{k=0}^{\mathrm{n}-1} \alpha_{0}^{k} \beta_{0} .
\end{aligned}
$$

The $\mathrm{n}$ solution of this relation is bounded for $\left|\alpha_{0}\right| \leq 1$, which implies that

$$
\begin{aligned}
-1 & \leq-\frac{\Delta t}{\tau_{m}}-\frac{\Delta t}{C_{m} D / 2 \sigma} \leq 1 \\
-2 & \leq-\frac{\Delta t}{\tau_{m}}-\frac{\Delta t}{C_{m} D / 2 \sigma} \leq 0 \\
0 & \leq \frac{\Delta t}{\tau_{m}}+\frac{\Delta t}{C_{m} D / 2 \sigma} \leq 2
\end{aligned}
$$

For biological parameters the expression in the middle is always positive so,

$$
\Delta t \leq \frac{2}{\frac{1}{\tau_{m}}+\frac{1}{C_{m} D / 2 \sigma}} .
$$

Some typical values are $C_{m}=1 \mu \mathrm{F} / \mathrm{cm}^{2}, \sigma=10 \mathrm{mS} / \mathrm{cm}, D=10 \mu \mathrm{m}, R_{m}=1 \mathrm{mS} / \mathrm{cm}^{2}$. A typical time restriction for the Euler forward method would be 100 nanoseconds

$$
\frac{2 \times 1 \frac{\mu F}{c m^{2}}}{\frac{2 \times 10 \frac{m S}{c m}}{1 \times 10^{-3} c m}+1 \frac{m S}{c m^{2}}} \approx 0.1 \mu s,
$$

showing the strictness of the time step requirement for the recurrence to converge. The term $C_{m} D / 2 \sigma$ is a time constant related to the cell time constant Eq. (3.7). In 
the numerical experiments the relation of this value to the convergence behavior can be observed in Figure 5.3. For biological cells this value is small relative to $\tau_{m}$. As $C_{m} D / 2 \sigma \ll \tau_{m}$ then one can approximate this criterion by:

$$
\Delta t<\frac{C_{m} D}{\sigma}
$$

For the Crank-Nicolson method the time stepping expression is,

$$
V_{m}^{\mathrm{n}+1}=V_{m}^{\mathrm{n}}+\frac{\Delta t}{C_{m}}\left(\left(\frac{I_{E}}{2}-\frac{V_{m}^{n+1}}{D / \sigma}+\frac{I_{E}}{2}-\frac{V_{m}^{n}}{D / \sigma}\right)-\frac{V_{m}^{n}}{R_{m}}\right)
$$

Expanding,

$$
V_{m}^{\mathrm{n}+1}=V_{m}^{\mathrm{n}}+\frac{\Delta t}{C_{m}} I_{E}-\frac{\Delta t}{C_{m} D / \sigma} V_{m}^{n+1}-\frac{\Delta t}{C_{m} D / \sigma} V_{m}^{n}-\Delta t \frac{V_{m}^{n}}{\tau_{m}}
$$

Which reorganized is

$$
\left(1+\frac{\Delta t}{C_{m} D / \sigma}\right) V_{m}^{n+1}=\left(1-\frac{\Delta t}{C_{m} D / \sigma}-\frac{\Delta t}{\tau_{m}}\right) V_{m}^{n}+\frac{\Delta t}{C_{m}} I_{E}
$$

This provides the restriction $\left|1-\frac{\Delta t}{C_{m} D / \sigma}-\frac{\Delta t}{\tau_{m}} / 1+\frac{\Delta t}{C_{m} D / \sigma}\right| \leq 1$, equivalent to

$$
-1-\frac{\Delta t}{C_{m} D / \sigma} \leq 1-\frac{\Delta t}{\tau_{m}}-\frac{\Delta t}{C_{m} D / \sigma} \leq 1+\frac{\Delta t}{C_{m} D / \sigma}
$$

Adding $-1+\frac{\Delta t}{C_{m} D / \sigma}$ to each expression, results in the constrain

$$
-2 \leq-\frac{\Delta t}{\tau_{m}} \leq 2 \frac{\Delta t}{C_{m} D / \sigma}
$$

for which $-C_{m} D / \sigma \leq 2 \tau_{m}$ is always true. The Crank-Nicolson imposes the time step restriction,

$$
\Delta t \leq 2 \tau_{m}
$$

The influence of $h$ was however still unknown. This required a simplified mesh that could be used to relate the $h$ term to the voltage solution. The next section presents this simplification of the solution domain.

\subsection{Regular discretization of the space/membrane equations}

A more accurate description of the stability restrictions of the space/membrane equations required considering the spatial parameter $h$. A simplified expression of the finite element form of the problem was necessary to ease the analysis. The finite element discretization was constructed for a regular two dimensional triangulation of typical element length $h$ (Figure 5.4). This discretization is shown now. 

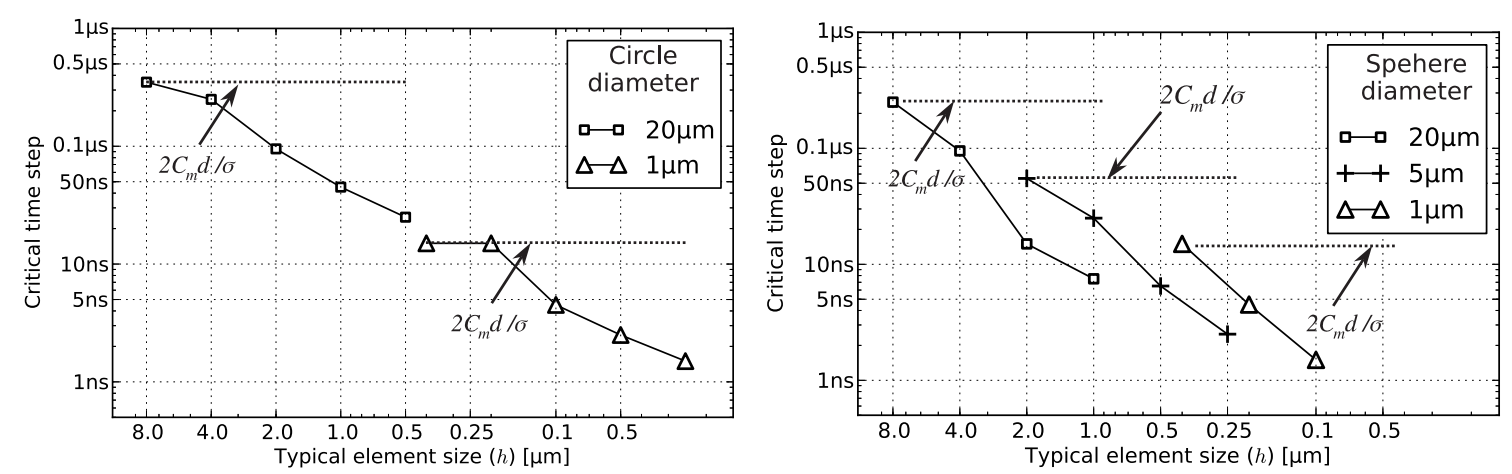

Figure 5.3: Stability restriction for the space independent recurrence relation. The doted horizontal line represents the time step limit as imposed by the space independent, infinite membrane approximation $\Delta t<2 \tau_{c}$.

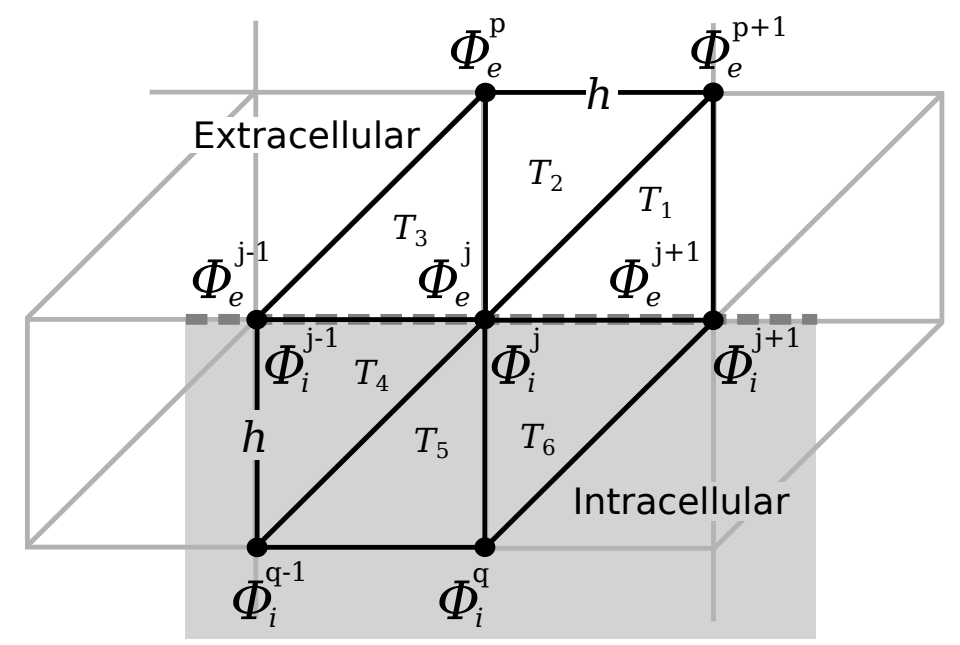

Figure 5.4: Section of a regular 2D triangulation used to estimate the relation between the maximum time step $\Delta t$ and the minimum space step $h$. The dashed horizontal line represents the membrane. Triangles above the membrane are named from right to left as $T_{1}, T_{2}$ and $T_{3}$. 
Beginning with the finite element form of Poisson's equation (4.7):

$$
\sigma \sum_{\mathrm{k}} \Phi^{\mathrm{k}} \int_{\Omega} \nabla v^{\mathrm{k}} \cdot \nabla v^{\mathrm{i}} d V+\sum_{\mathrm{j}} I_{m}^{\mathrm{j}} \int_{\Gamma} u^{\mathrm{j}}\left[v^{\mathrm{i}}\right]_{i, e} d S=\int_{\Omega} s v^{\mathrm{i}} d V
$$

for an exterior interface node $\mathrm{j}$ in $\Gamma$ with corresponding spatial test function $v^{\mathrm{i}}$ in $\Omega_{e} \rightarrow$ $v^{\mathrm{j}}$ in $\Omega_{e} \cap \Gamma$, assuming no current source or boundary condition touches the membrane, and using the assumption $u^{\mathrm{j}}=1^{1}$ one has

$$
\sigma \sum_{\mathrm{k}} \Phi_{e}^{\mathrm{k}} \int_{\Omega} \nabla v^{\mathrm{k}} \cdot \nabla v^{\mathrm{j}} d V+I_{m}^{\mathrm{j}} \int_{\Gamma} v^{\mathrm{j}} d S=0
$$

The membrane current can be extracted as

$$
I_{m}^{\mathrm{j}}=-\sigma \frac{\sum_{\mathrm{k}} \Phi_{e}^{\mathrm{k}} \int_{\Omega} \nabla v^{\mathrm{k}} \cdot \nabla v^{\mathrm{j}} d V}{-\int_{\Gamma} v^{\mathrm{j}} d S}=-\sigma \frac{S_{0}}{S_{1}} .
$$

Introducing the abbreviated terms $S_{0}$ and $S_{1}$ for the sum in the numerator and the integral in the denominator respectively. For the following analyses, the membrane current term needs to be expressed in terms of the membrane voltage $V_{m}$. This can be achieved by partially reducing the integral term $S_{0}$ to membrane voltage values. For the triangles $T_{1}, T_{2}$ and $T_{3}$ of the regular mesh of Figure 5.4 this term is

$$
S_{0}=\int_{T_{1}} \sum_{\mathrm{k}} \Phi^{\mathrm{k}} \nabla v^{\mathrm{k}} \cdot \nabla v^{\mathrm{j}} d V+\int_{T_{2}} \sum_{\mathrm{k}} \Phi^{\mathrm{k}} \nabla v^{\mathrm{k}} \cdot \nabla v^{\mathrm{j}} d V+\int_{T_{3}} \sum_{\mathrm{k}} \Phi^{\mathrm{k}} \nabla v^{\mathrm{k}} \cdot \nabla v^{\mathrm{j}} d V
$$

Expanding the sums

$$
\begin{aligned}
S_{0}= & \int_{T_{1}}\left(\Phi^{\mathrm{j}+1} \nabla v^{\mathrm{j}+1}+\Phi^{\mathrm{p}+1} \nabla v^{\mathrm{p}+1}+\Phi^{\mathrm{j}} \nabla v^{\mathrm{j}}\right) \cdot \nabla v^{\mathrm{j}} d V \\
& +\int_{T_{2}}\left(\Phi^{\mathrm{p}+1} \nabla v^{\mathrm{p}+1}+\Phi^{\mathrm{p}} \nabla v^{\mathrm{p}}+\Phi^{\mathrm{j}} \nabla v^{\mathrm{j}}\right) \cdot \nabla v^{\mathrm{j}} d V \\
& +\int_{T_{3}}\left(\Phi^{\mathrm{p}} \nabla v^{\mathrm{p}}+\Phi^{\mathrm{j}-1} \nabla v^{\mathrm{j}-1}+\Phi^{\mathrm{j}} \nabla v^{\mathrm{j}}\right) \cdot \nabla v^{\mathrm{j}} d V
\end{aligned}
$$

and replacing with the simple "hat" test function derivatives:

$$
\begin{aligned}
S_{0}= & \int_{T_{1}}\left(\Phi^{\mathrm{j}+1}\left[\begin{array}{ll}
\frac{1}{h} & -\frac{1}{h}
\end{array}\right]+\Phi^{\mathrm{p}+1}\left[\begin{array}{ll}
0 & \frac{1}{h}
\end{array}\right]+\Phi^{\mathrm{j}}\left[\begin{array}{ll}
-\frac{1}{h} & 0
\end{array}\right]\right) \cdot\left[\begin{array}{c}
-\frac{1}{h} \\
0
\end{array}\right] d V \\
& +\int_{T_{2}}\left(\Phi^{\mathrm{p}+1}\left[\begin{array}{ll}
\frac{1}{h} & 0
\end{array}\right]+\Phi^{\mathrm{p}}\left[\begin{array}{ll}
-\frac{1}{h} & \frac{1}{h}
\end{array}\right]+\Phi^{\mathrm{j}}\left[\begin{array}{ll}
0 & -\frac{1}{h}
\end{array}\right]\right) \cdot\left[\begin{array}{c}
0 \\
-\frac{1}{h}
\end{array}\right] d V \\
& +\int_{T_{3}}\left(\Phi^{\mathrm{p}}\left[\begin{array}{ll}
0 & \frac{1}{h}
\end{array}\right]+\Phi^{\mathrm{j}-1}\left[\begin{array}{ll}
-\frac{1}{h} & 0
\end{array}\right]+\Phi^{\mathrm{j}}\left[\begin{array}{ll}
\frac{1}{h} & -\frac{1}{h}
\end{array}\right]\right) \cdot\left[\begin{array}{c}
\frac{1}{h} \\
-\frac{1}{h}
\end{array}\right] d V .
\end{aligned}
$$

\footnotetext{
${ }^{1}$ This is a common assumption in implementations of the Neumann boundary condition. Although the membrane current term is not strictly a Neumann condition, it has the same form. This assumption was also made in Eq. 4.1a of Ying and Henriquez paper [37].
} 
Factorizing

$$
S_{0}=\int_{T_{1}}\left(-\frac{\Phi^{\mathrm{j}+1}}{h^{2}}+\frac{\Phi^{\mathrm{j}}}{h^{2}}\right) d V+\int_{T_{2}}\left(-\frac{\Phi^{\mathrm{p}}}{h^{2}}+\frac{\Phi^{\mathrm{j}}}{h^{2}}\right) d V+\int_{T_{3}}\left(-\frac{\Phi^{\mathrm{p}}}{h^{2}}-\frac{\Phi^{\mathrm{j}-1}}{h^{2}}+\frac{2 \Phi^{\mathrm{j}}}{h^{2}}\right) d V .
$$

The $\Phi$ and $h$ terms are coefficients in the finite element method so the volumes in two-dimensions are the areas

$$
S_{0}=\left(-\frac{\Phi^{\mathrm{j}+1}}{h^{2}}+\frac{\Phi^{\mathrm{j}}}{h^{2}}\right)\left|T_{1}\right|+\left(-\frac{\Phi^{\mathrm{p}}}{h^{2}}+\frac{\Phi^{\mathrm{j}}}{h^{2}}\right)\left|T_{2}\right|+\left(-\frac{\Phi^{\mathrm{p}}}{h^{2}}-\frac{\Phi^{\mathrm{j}-1}}{h^{2}}+\frac{2 \Phi^{\mathrm{j}}}{h^{2}}\right)\left|T_{3}\right| .
$$

Areas for the regular grid are $\left|T_{1}\right|=\left|T_{2}\right|=\left|T_{3}\right|=\frac{h^{2}}{2}$ implying

$$
\begin{aligned}
S_{0} & =\left(-\frac{\Phi^{\mathrm{j}+1}}{2}+\frac{\Phi^{\mathrm{j}}}{2}\right)+\left(-\frac{\Phi^{\mathrm{p}}}{2}+\frac{\Phi^{\mathrm{j}}}{2}\right)+\left(-\frac{\Phi^{\mathrm{p}}}{2}-\frac{\Phi^{\mathrm{j}-1}}{2}+\Phi^{\mathrm{j}}\right) \\
& =-\frac{1}{2} \Phi^{\mathrm{j}+1}-\Phi^{\mathrm{p}}-\frac{1}{2} \Phi^{\mathrm{j}-1}+2 \Phi^{\mathrm{j}}
\end{aligned}
$$

The term $S_{1}$ in $(5.6)$ is

$$
S_{1}=\int_{\Gamma} v^{\mathrm{j}} d s=\int_{L_{1}} v^{\mathrm{j}} d S+\int_{L_{3}} v^{\mathrm{j}} d S=\frac{h}{2}+\frac{h}{2}=h
$$

The extracellular membrane current in the regular finite element discretization is then

$$
I_{m}^{\mathrm{j}}=-\frac{\sigma}{h}\left(\frac{1}{2} \Phi_{e}^{\mathrm{j}-1}+\frac{1}{2} \Phi_{e}^{\mathrm{j}+1}+\Phi_{e}^{\mathrm{p}}-2 \Phi_{e}^{\mathrm{j}}\right),
$$

and a similar procedure leads to the intracellular expression

$$
-I_{m}^{\mathrm{j}}=-\frac{\sigma}{h}\left(\frac{1}{2} \Phi_{i}^{\mathrm{j}-1}+\frac{1}{2} \Phi_{i}^{\mathrm{j}+1}+\Phi_{i}^{\mathrm{q}}-2 \Phi_{i}^{\mathrm{j}}\right) .
$$

Subtracting this two

$$
2 I_{m}^{\mathrm{j}}=-\frac{\sigma}{h}\left(-\frac{1}{2} \Phi_{i}^{\mathrm{j}-1}-\frac{1}{2} \Phi_{i}^{\mathrm{j}+1}-\Phi_{i}^{\mathrm{q}}+2 \Phi_{i}^{\mathrm{j}}+\frac{1}{2} \Phi_{e}^{\mathrm{j}-1}+\frac{1}{2} \Phi_{e}^{\mathrm{j}+1}+\Phi_{e}^{\mathrm{p}}-2 \Phi_{e}^{\mathrm{j}}\right)
$$

and considering that the membrane voltage is $V_{m}^{\mathrm{j}}=\Phi_{i}^{\mathrm{j}}-\Phi_{e}^{\mathrm{j}}$

$$
I_{m}^{\mathrm{j}}=-\frac{\sigma}{h}\left(-\frac{1}{4} \Phi_{i}^{\mathrm{j}-1}-\frac{1}{4} \Phi_{i}^{\mathrm{j}+1}-\frac{1}{2} \Phi_{i}^{\mathrm{q}}+\Phi_{i}^{\mathrm{j}}+\frac{1}{4} \Phi_{e}^{\mathrm{j}-1}+\frac{1}{4} \Phi_{e}^{\mathrm{j}+1}+\frac{1}{2} \Phi_{e}^{\mathrm{p}}-\Phi_{e}^{\mathrm{j}}\right) .
$$

An expression for $I_{m}$ depending on $V_{m}$ is

$$
I_{m}^{\mathrm{j}}=-\frac{\sigma}{h}\left(V_{m}^{\mathrm{j}}-\frac{1}{4} V_{m}^{\mathrm{j}-1}-\frac{1}{4} V_{m}^{\mathrm{j}+1}-\frac{1}{2} \Phi_{i}^{\mathrm{q}}+\frac{1}{2} \Phi_{e}^{\mathrm{p}}\right)
$$




\subsection{Space dependent recurrence relation criterion}

An approach combining the recurrence relation analysis and the discrete current expression (5.9) was used to obtain a first approximation to the stability criterion depending on $h$. This approximation ignores the influence of neighboring non-membrane nodes which are lumped in a constant term $K\left(V_{m}^{\mathrm{i} \neq \mathrm{j}}, \Phi_{\Omega-\Gamma}\right)$. The von Neumann analysis will consider the effect of the neighboring membrane nodes $V_{m}^{\mathrm{i} \neq \mathrm{j}}$ and see Section 5.7 for a discussion regarding the $\Phi_{\Omega-\Gamma}$ terms. Dropping the spatial index, the membrane current is then

$$
I_{m}=-\frac{\sigma}{h} V_{m}+K
$$

The forward Euler (4.24) expression combined with (5.10) is

$$
\begin{aligned}
V_{m}^{\mathrm{n}+1} & =V_{m}^{\mathrm{n}}+\frac{\Delta t}{C_{m}}\left(I_{m}^{\mathrm{n}}-I_{i o n}^{\mathrm{n}}\right) \\
& =V_{m}^{\mathrm{n}}+\frac{\Delta t}{C_{m}}\left(-\frac{\sigma}{h} V_{m}^{\mathrm{n}}+K-\frac{V_{m}^{\mathrm{n}}}{R_{m}}\right) \\
& =\left(1-\frac{\Delta t}{C_{m}} \frac{\sigma}{h}-\frac{\Delta t}{\tau_{m}}\right) V_{m}+\frac{\Delta t}{C_{m}} K
\end{aligned}
$$

As in (5.2) the series converges for the range

$$
\begin{aligned}
-1 & \leq 1-\frac{\Delta t}{C_{m}} \frac{\sigma}{h}-\frac{\Delta t}{\tau_{m}} \leq 1 \\
-2 & \leq-\frac{\Delta t}{C_{m}} \frac{\sigma}{h}-\frac{\Delta t}{\tau_{m}} \leq 0 \\
0 & \leq \frac{\Delta t}{C_{m}} \frac{\sigma}{h}+\frac{\Delta t}{\tau_{m}} \leq 2
\end{aligned}
$$

and the stability criterion is

$$
\Delta t \leq \frac{2}{\frac{\sigma}{C_{m} h}+\frac{1}{\tau_{m}}}
$$

or approximately

$$
\Delta t<2 \frac{C_{m}}{\sigma} h
$$

This stability criterion for the experiments in Figure 5.2 is shown in Figure 5.5. The parameter is not really different from the cell time constant 3.7 differing only in the 2 coefficient and diameter. This points to a time restriction given now not by the cell diameter but from the size of the solution mesh element.

As will be seen next the Crank-Nicolson method remains as $\Delta t<2 \tau_{m}$. The time iteration expression is

$$
\begin{aligned}
V_{m}^{\mathrm{n}+1} & =V_{m}^{\mathrm{n}}+\frac{\Delta t}{C_{m}}\left(\frac{1}{2}\left(I_{m}^{\mathrm{n}+1}+I_{m}^{\mathrm{n}}\right)-I_{i o n}^{\mathrm{n}}\right) \\
& =V_{m}^{\mathrm{n}}+\frac{\Delta t}{C_{m}}\left(-\frac{\sigma}{2 h} V_{m}^{\mathrm{n}+1}+\frac{K}{2}-\frac{\sigma}{2 h} V_{m}^{\mathrm{n}}+\frac{K}{2}-\frac{V_{m}^{\mathrm{n}}}{R_{m}}\right) \\
\left(1+\frac{\Delta t}{2 C_{m}} \frac{\sigma}{h}\right) V_{m}^{\mathrm{n}+1} & =\left(1-\frac{\Delta t}{2 C_{m}} \frac{\sigma}{h}-\frac{\Delta t}{\tau_{m}}\right) V_{m}^{\mathrm{n}}+\frac{\Delta t}{C_{m}} K
\end{aligned}
$$



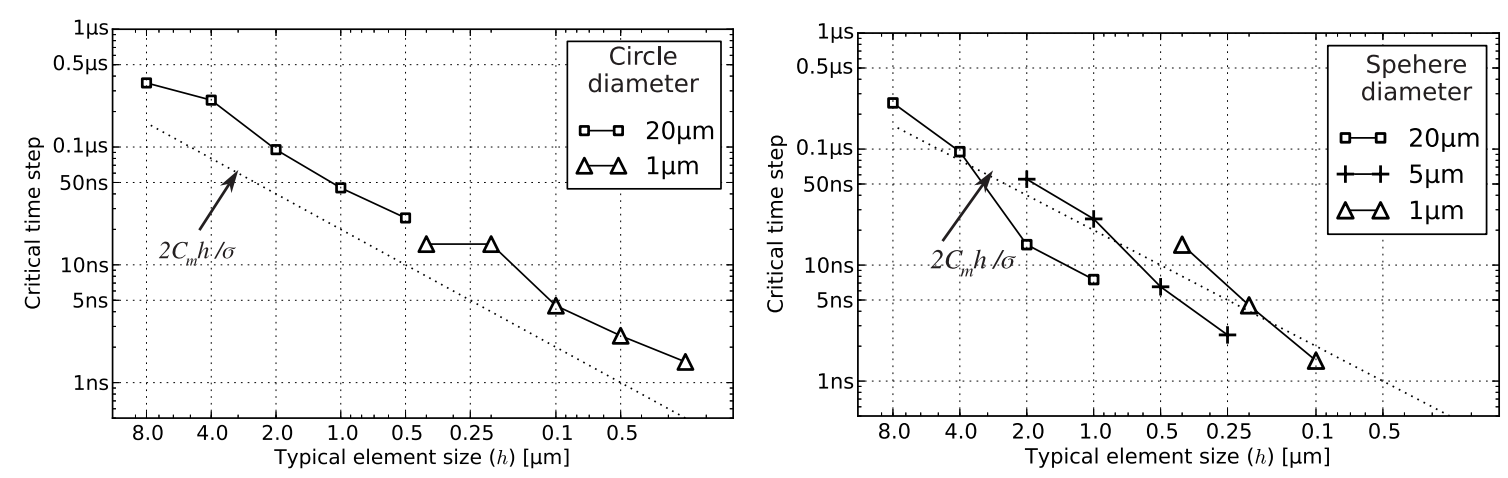

Figure 5.5: Stability restriction for the space dependent recurrence relation. The doted line represents the time step limit as imposed by the space dependent approximation $\Delta t<2 \frac{C_{m}}{\sigma} h$.

For the series to converge $\left|\frac{1-\frac{\Delta t}{2 C_{m}} \frac{\sigma}{h}-\frac{\Delta t}{\tau_{m}}}{1+\frac{\Delta t}{2 C_{m}} \frac{\sigma}{h}}\right| \leq 1$ so

$$
-1-\frac{\Delta t}{2 C_{m}} \frac{\sigma}{h} \leq 1-\frac{\Delta t}{2 C_{m}} \frac{\sigma}{h}-\frac{\Delta t}{\tau_{m}} \leq 1+\frac{\Delta t}{2 C_{m}} \frac{\sigma}{h}
$$

Adding $-1+\frac{\Delta t}{2 C_{m}} \frac{\sigma}{h}$ to the expression,

$$
-2 \leq-\frac{\Delta t}{\tau_{m}} \leq \frac{\Delta t}{C_{m}} \frac{\sigma}{h}
$$

Two constrains result. One is

$$
\begin{aligned}
-\frac{\Delta t}{\tau_{m}} & \leq \frac{\Delta t}{C_{m}} \frac{\sigma}{h} \\
-C_{m} \frac{h}{\sigma} & \leq \tau_{m}
\end{aligned}
$$

which is always true, and

$$
\Delta t \leq 2 \tau_{m}
$$

which is identical to (5.4) and $h$ independent.

\section{6 von Neumann stability analysis}

The von Neumann stability analysis shows a more conclusive stability restriction that includes the influence of the neighboring membrane potential terms. For the forward Euler case, from (4.24) and (5.9)

$$
\begin{aligned}
V_{m}^{\mathrm{j}, \mathrm{n}+1} & =V_{m}^{\mathrm{j}, \mathrm{n}}+\frac{\Delta t}{C_{m}}\left(I_{m}^{\mathrm{j}, \mathrm{n}}-I_{i o n}^{\mathrm{j}, \mathrm{n}}\right) \\
& =V_{m}^{\mathrm{j}, \mathrm{n}}+\frac{\Delta t}{C_{m}}\left(-\frac{\sigma}{h}\left(V_{m}^{\mathrm{j}, \mathrm{n}}-\frac{1}{4} V_{m}^{\mathrm{j}-1, \mathrm{n}}-\frac{1}{4} V_{m}^{\mathrm{j}+1, \mathrm{n}}-\frac{1}{2} \Phi_{i}^{\mathrm{q}, \mathrm{n}}+\frac{1}{2} \Phi_{e}^{\mathrm{p}, \mathrm{n}}\right)-\frac{1}{R_{m}} V_{m}^{\mathrm{j}, \mathrm{n}}\right)
\end{aligned}
$$


Expanding and factorizing again

$$
\begin{aligned}
V_{m}^{\mathrm{j}, \mathrm{n}+1}= & \left(1-\frac{\Delta t}{C_{m}} \frac{1}{R_{m}}-\frac{\Delta t}{C_{m}} \frac{\sigma}{h}\right) V_{m}^{\mathrm{j}, \mathrm{n}}+ \\
& \frac{\Delta t}{C_{m}} \frac{\sigma}{h} \frac{1}{4} V_{m}^{\mathrm{j}-1, \mathrm{n}}+\frac{\Delta t}{C_{m}} \frac{\sigma}{h} \frac{1}{4} V_{m}^{\mathrm{j}+1, \mathrm{n}}+\frac{\Delta t}{C_{m}} \frac{\sigma}{h} \frac{1}{2}\left(\Phi_{i}^{\mathrm{q}, \mathrm{n}}-\Phi_{e}^{\mathrm{p}, \mathrm{n}}\right) .
\end{aligned}
$$

Or in a lighter notation

$$
\begin{aligned}
V_{m}^{\mathrm{j}, \mathrm{n}+1} & =(1-\alpha-\beta) V_{m}^{\mathrm{j}, \mathrm{n}}+\frac{\beta}{4}\left(V_{m}^{\mathrm{j}-1, \mathrm{n}}+V_{m}^{\mathrm{j}+1, \mathrm{n}}\right)+\psi \\
\alpha & =\frac{\Delta t}{C_{m}} \frac{1}{R_{m}}, \quad \beta=\frac{\sigma \Delta t}{C_{m} h}, \quad \psi=\frac{\Delta t}{C_{m}} \frac{\sigma}{h} \frac{1}{2}\left(\Phi_{i}^{\mathrm{q}, \mathrm{n}}-\Phi_{e}^{\mathrm{p}, \mathrm{n}}\right) .
\end{aligned}
$$

The standard ansatz of the von Neumann stability analysis [104] can now be used:

$$
V_{m}^{\mathrm{j}, \mathrm{n}}=e^{\mathrm{i} \gamma \mathrm{j} h}
$$

With $\mathrm{j}, \mathrm{n}$ the space and time indexes, $\mathrm{i}$ the imaginary unit, and $\gamma$ a variable wave number.

Again the influence of the potential terms in $\psi$ is disregarded. See Section 5.7 for a discussion on this topic. Replacing in 5.12

$$
V_{m}^{\mathrm{j}, \mathrm{n}+1}=(1-\alpha-\beta) e^{\mathrm{i} \gamma \mathrm{j} h}+\frac{\beta}{4}\left(e^{\mathrm{i} \gamma(\mathrm{j}-1) h}+e^{\mathrm{i} \gamma(\mathrm{j}+1) h}\right)+\psi,
$$

expanding the exponential term, using the identity $e^{-\mathrm{i} \gamma h}+e^{\mathrm{i} \gamma h}=2 \cos \gamma h$ and factorizing

$$
\begin{aligned}
V_{m}^{\mathrm{j}, \mathrm{n}+1} & =\left(1-\alpha-\beta+\frac{\beta}{2} \cos \gamma h\right) e^{\mathrm{i} \gamma \mathrm{j} h}+\psi \\
& =\xi e^{\mathrm{i} \gamma \mathrm{j} h}+\psi
\end{aligned}
$$

with $\xi$ symbolizing the von Neumann amplification factor. The amplification factor can be converted to the more convenient square sine form

$$
\begin{aligned}
\xi & =1-\alpha-\beta+\frac{\beta}{2} \cos \gamma h \\
& =1-\alpha-\beta+\frac{\beta}{2}\left(1-2 \sin ^{2} \frac{\gamma h}{2}\right) \\
& =1-\alpha-\frac{\beta}{2}-\beta \sin ^{2} \frac{\gamma h}{2} .
\end{aligned}
$$


Expanding the above expression,

$$
\begin{aligned}
V_{m}^{1} & =\xi V_{m}^{0}+\psi \\
V_{m}^{2} & =\xi\left(\xi V_{m}^{0}+\psi\right)+\psi=\xi^{2} V_{m}^{0}+\xi \psi+\psi \\
V_{m}^{3} & =\xi\left(\xi^{2} V_{m}^{0}+\xi \psi+\psi\right)+\psi=\xi^{3} V_{m}^{0}+\xi^{2} \psi+\xi \psi+\psi \\
V_{m}^{4} & =\xi\left(\xi^{3} V_{m}^{0}+\xi^{2} \psi+\xi \psi+\psi\right)+\psi=\xi^{4} V_{m}^{0}+\xi^{3} \psi+\xi^{2} \psi+\xi \psi+\psi \\
& \vdots \\
V_{m}^{\mathrm{p}} & =\xi^{\mathrm{p}} V_{m}^{0}+\sum_{l=0}^{\mathrm{p}-1} \xi^{l} \psi
\end{aligned}
$$

If $p \rightarrow \infty$ the solution is bounded only for $|\xi|<1$

$$
\begin{aligned}
-1 & \leq 1-\alpha-\frac{\beta}{2}-\beta \sin ^{2} \frac{\gamma h}{2} \leq 1 \\
-2 & \leq-\alpha-\frac{\beta}{2}-\beta \sin ^{2} \frac{\gamma h}{2} \leq 0 \\
0 & \leq \alpha+\frac{\beta}{2}+\beta \sin ^{2} \frac{\gamma h}{2} \leq 2
\end{aligned}
$$

The term in the middle is always positive so

$$
\alpha+\frac{\beta}{2}+\beta \sin ^{2} \frac{\gamma h}{2} \leq 2
$$

Taking $\max \left(\sin ^{2} \frac{\gamma h}{2}\right)=1$ and replacing $\alpha$ and $\beta$ back

$$
\begin{aligned}
\frac{\Delta t}{C_{m}} \frac{1}{R_{m}}+\frac{3}{2} \frac{\Delta t}{C_{m}} \frac{\sigma}{h} & \leq 2 \\
\frac{\Delta t}{C_{m}}\left(\frac{1}{R_{m}}+\frac{3}{2} \frac{\sigma}{h}\right) & \leq 2 \\
\Delta t & \leq \frac{2}{\frac{1}{R_{m} C_{m}}+\frac{3}{2} \frac{\sigma}{C_{m} h}}
\end{aligned}
$$

As before $C_{m} h / \sigma \ll R_{m} C_{m}$ so the condition can be approximated by

$$
\Delta t<\frac{4 C_{m} h}{3 \sigma} .
$$

This stability criterion is $h^{1}$ dependent and differs from (5.11) only in the numeric coefficient. These two criteria are compared in Figure 5.6 for the experiments in Figure 5.2 .

The restriction on the time-step is again laxer in the Crank-Nicolson scheme. Inserting (5.9) in the scheme (4.25) expands to

$$
\begin{aligned}
\left(1+\frac{\beta}{2}\right) V_{m}^{\mathrm{j}, \mathrm{n}+1}-\frac{\beta}{8}\left(V_{m}^{\mathrm{j}-1, \mathrm{n}+1}+V_{m}^{\mathrm{j}+1, \mathrm{n}+1}\right)= & \left(1-\frac{\beta}{2}-\alpha\right) V_{m}^{\mathrm{j}, \mathrm{n}}+ \\
& \frac{\beta}{8}\left(V_{m}^{\mathrm{j}-1, \mathrm{n}}+V_{m}^{\mathrm{j}+1, \mathrm{n}}\right)-\psi^{\mathrm{n}+1}-, \psi^{\mathrm{n}}
\end{aligned}
$$



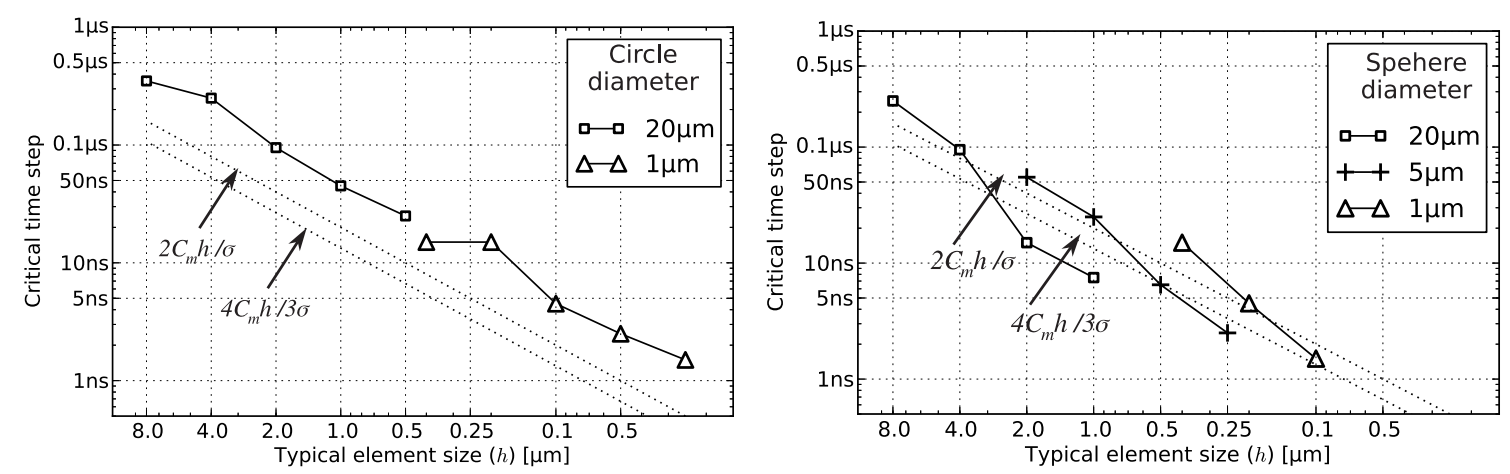

Figure 5.6: Stability restriction on the space dependent von Neumann analysis compared to the recurrence analysis. The doted line represents the time step limit as imposed by the space dependent approximations $\Delta t<2 \frac{C_{m}}{\sigma} h$ and $\Delta t<\frac{4 C_{m} h}{3 \sigma}$.

The amplification factor is

$$
\xi_{C N}=\frac{1-\frac{\beta}{2}\left(\frac{1}{2}+\sin ^{2} \frac{\gamma h}{2}\right)-\alpha}{1+\frac{\beta}{2}\left(\frac{1}{2}+\sin ^{2} \frac{\gamma h}{2}\right)} .
$$

Adding $-1+\frac{\beta}{2}\left(\frac{1}{2}+\sin ^{2} \frac{\gamma h}{2}\right)$ to the inequality $-1 \leq \xi_{C N} \leq 1$ gives

$$
-\beta\left(\frac{1}{2}+\sin ^{2} \frac{\gamma h}{2}\right) \leq \alpha \leq 2
$$

$\alpha$ and $\beta$ are always positive so the left inequality is always true. The right part establishes again that the stability limits are set by membrane time constant $\tau_{m}=R_{m} C_{m}$ and are $h$ independent:

$$
\Delta t \leq 2 \tau_{m}
$$

\subsection{Discussion}

The previous analyses showed stability under strict requirements for the Euler method (approaching the cell time constant) and more relaxed requirements for the CrankNicolson. The Crank-Nicolson scheme is not unconditionally stable (e.g. as in the diffusion equation) but its stability is only restricted by the millisecond long, membrane time constant. This shows the robustness of the method allowing simulations with relatively large time steps. As in typical simulations, faster dynamics such as those from ion channels are involved, a time step of $2 \tau_{m}$ is more that enough for practical cases.

It is important to discuss the dropping of the spatial potential terms in the previous approximations (Sections 5.5 and 5.6). The potential is an unknown of the solution and the spread of instability to these points might affect the overall result. Treatment of 
points outside the membrane is however not simple as the von Neumann ansatz would have to be extended to a 2D spatial form. This analysis was not performed. Given that the restriction terms 5.11 and 5.14 only differ by coefficients, it is tempting to believe that adding the potential points would only affect the coefficient of the restriction term. It is clear that numerically, for at least the $2 \mathrm{D}$ simulation, the stability term only differs by coefficient from the analytical results (see for instance Figure 5.6). The order of the stability restriction should not be larger than 1 for $2 \mathrm{D}$ and, as will be shown next, this seems also to be true for the $3 \mathrm{D}$ case.

The variations of the stability trace in the $3 \mathrm{D}$ experiments with respect to the analytical estimate can be explained by a change in coefficient and by the statistical nature of the meshing algorithm. Reducing the variability of the 3D mesh shows a clear $h^{1}$ stability restriction. This can be seen in Figure 5.7 where a regularly, meshed cubic cell was simulated. The stability trace did not match alternative $h^{2}$ or $h^{3}$ criteria.

In the regular case there is not statistical variation of the mesh size. In irregular meshes such as those of Figure 5.1 the approximation of the criterion (Figure 5.6) is not as precise as in Figure 5.7. Compliance to an $h$ parameter given to a discretization algorithm is not exact in irregular meshes, as different meshing algorithms have to sacrifice minimal length to increase accuracy of the representation. The triangles or tetrahedra actually follow a distribution, and for the particular discretization tool used (GMSH, Section 6.3), the meshing distribution seems to be particularly worst in 3D.

The tetrahedral volume distribution of the 3D mesh in Figure 5.1 is shown in Figure 5.8. In this case the meshing algorithm was given a parameter $h=1$ to discretize the sphere. The volume of a regular tetrahedron of side $h=1$ is given by

$$
V o l=\frac{\sqrt{2}}{12} h^{3}=0.12
$$

which is the point where the distribution centers. Still, other smaller volumes are seen showing that the $h$ parameter is not consistent.

An additional clue pointing to an $h^{1}$ dependence in the 3D case is found in the voltage dependent, current term (5.9). The $h$ term in this expression is the one carried over to the stability restriction and its order can be inferred for a 3D discretization. The $h$ term in Eq. (5.9) results from the area and line integrals of Eq. (5.6) and the Laplacian. In an irregular three-dimensional mesh with a typical element of volume $V o l_{\Omega}^{h}$ and a typical membrane element of area $A r e a_{\Gamma}^{h}$ the current term should depend on $\operatorname{Vol}_{\Omega}^{h} / h^{2} A r e a_{\Omega}^{h}$. Approximating the volume by $h^{3}$ and the area by $h^{2}$ leaves a $1 / h$ term as in the $2 \mathrm{D}$ case. These operations also keep the physical units consistent.

\subsection{Summary}

This chapter validated the numerical strategy presented in Chapter 4 through stability analysis. Validating of a PDE solution requires demonstrating stability, consistency and convergence but from the Lax-Ritchtmyer theorem stability and consistency directly imply convergence. The methods of Chapter 4 are known to be consistent and stability under the time step restrictions presented here is achievable. Showing stability for the 

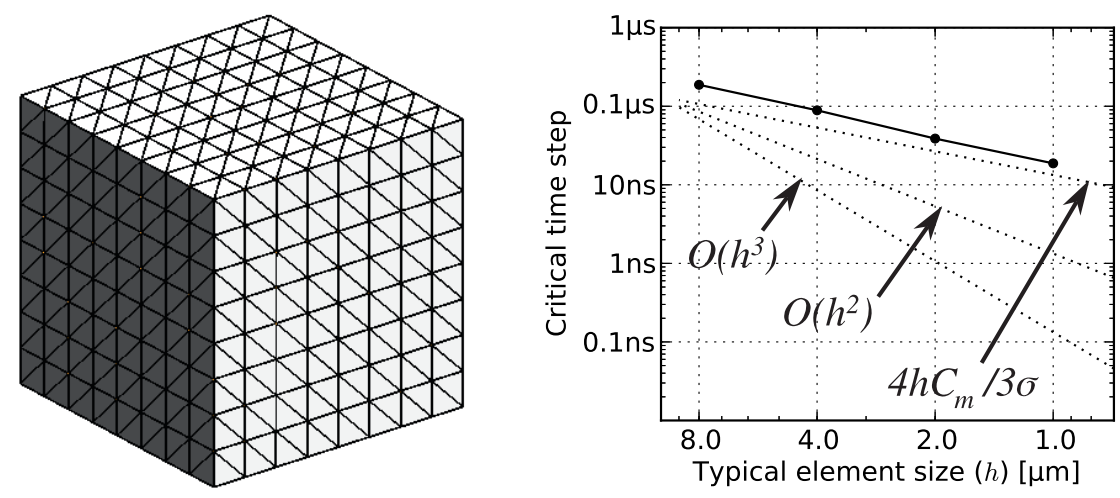

Figure 5.7: Experimental stability criterion results for a $l=16 \mu \mathrm{m}$ cubic cell. The same stimulation parameters of the previous experiments were used. Doted lines represent the von Neumann criterion and alternatively, how an $O\left(h^{2}\right)$ and $O\left(h^{3}\right)$ dependence would look like.
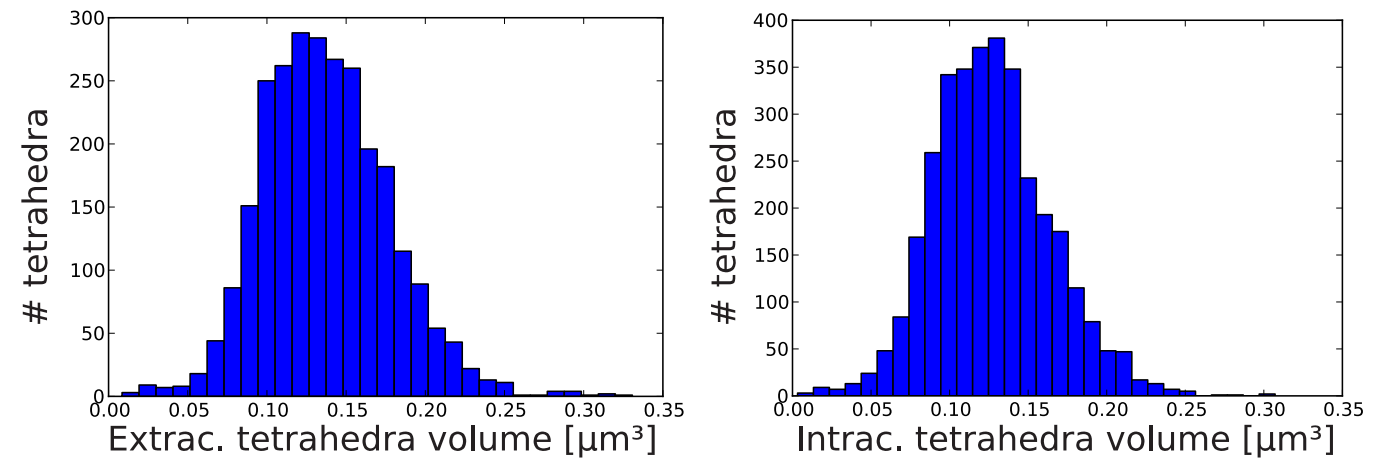

Figure 5.8: Distribution of tetrahedra volume for a spherical cell mesh. Parameter $h=1$ was given to the meshing algorithm (GMSH Delaunay 3D algorithm). For a regular tetrahedron of side $h=1$ the expected volume is 0.12 . 
numerical approach was complicated by the discontinuity of the cell membrane. While the main time evolution domain in continuous cases (e.g. the diffusion equation) is the entire solution space in the space/membrane equations this happens only on the membrane. The stability analysis was approximated analytically and comparisons were made to the numerical case.

The numerical experiments mostly matched the analytical approximation and the coefficient variations to the stability trace were partially explained. In conclusion the following factors contribute to the analytical and numerical difference:

- Mesh variability.

- Use or not of the voltage terms in the $h$ dependent approach.

- Errors introduced by over simplification of the finite element discretization.

Regardless of these differences it is highly likely that the time step restriction is $h^{1}$ dependent for the 2D and 3D cases.

It should be mentioned that the Crank-Nicolson, the preferred method, did not show any dependence on $h$. Stability dependence on the spatial mesh parameter $h$ appeared in the Euler but not in the Crank-Nicolson restriction. Numerous other experiments (e.g. in Chapter 7) corroborate this robustness. 


\section{Numerical method implementation: the CHASTE-Membrane tool}

\subsection{Introduction}

A result of this work was the construction of a computer tool for the simulation of arbitrarily shaped cells and their intra- and extra-cellular space. The tool, which took the name CHASTE-Membrane ${ }^{1}$ is based in the biophysical fundamentals presented in Chapter 2 and the numerical methods presented in Chapter 4. To my knowledge, a complete open solver for the space/membrane equations did not exist before. CHASTEMembrane is now freely available to the scientific community ${ }^{2}$. This chapter presents key details about the design and construction of the tool.

\subsubsection{State of the art}

Numerous research and engineering applications require the solution of partial differential equations (PDEs) over non-analytically tractable domains. In these cases the PDEs need to be solved with computer programs such as finite element method (FEM) solvers. FEM solvers are not trivial programs and in general cannot be written as simple MATLAB scripts. For the treatment of potentially large problems, these programs require simultaneously to be efficient, to support parallelization, to be simple to use, and to be flexible upon parameter and equation changes. High performance also requires advanced system languages such as $\mathrm{C}$ or $\mathrm{C}++$.

For these and other reasons the construction of such tools is expensive and many laboratories opt for buying or using generic FEM packages. Multiple computing environments solving general FEM problems exist. Two representative commercial solvers are COMSOL [118] and ANSYS [119]. Free alternatives to these are FreeFem $++[120]$ and FEniCS [121].

These packages are designed for the analysis of generic physical problems and in many cases the basic equations they provide can be crafted to solve more specific problems. However many physical problems cannot be covered by generically available

\footnotetext{
${ }^{1}$ CHASTE stands for "Cancer, Heart and Soft-Tissue Environment" and is an open source project mainly developed at the Computational Biology Group of Oxford University [117]. CHASTE-Membrane is now a sub-module of CHASTE that allows simulation of cell membranes. This functionality was not available in CHASTE before. More details about this environment are provided in this chapter.

${ }^{2}$ The CHASTE-Membrane source code was released under a GNU-LGPL open source license and can be downloaded from Oxford's Computational Biology Group website http://www.cs.ox.ac.uk/chaste
} 
FEM packages. An example of this is simulation of the heart electrophysiology [98] which has special conditions as the overlapping bidomain and non-standard operator splitting requirements. These specialized functions are rarely available in generic FEM solvers and in many cases, customized solvers have to be written. Fortunately for heart researchers several free packages exist. Example software for the solution of the bidomain equations are CARP [122], Continuity [123], CHASTE [117], among others [54].

The picture for the solution of the space/membrane equations is not as positive. Before CHASTE-Membrane, no commercial or open source packages existed for the solution of these equations. Although attempts had been made to solve these with crafted commercial solvers [35, 39, 52], they present numerical restrictions (see Chapter 4) and required expensive software licenses. For the reasons explained in chapters 2 and 4 the set of space/membrane equations differs from the classic Poisson's or the diffusion equation and present a strong discontinuity on the membrane.

Other closed-source approaches exist around Xylouris, Ying and colleagues [53, 38, 37]. After collaborating with Xylouris and co-workers partial access was granted to their tool $[38,53]$. However the lack of documentation, lack of standard mesh formats, and the fundamental numerical issues of their code (e.g. use of a Newton solver) lead to abandonment of this effort after two years of work. Although attempted, an agreement to use the code of Henriquez and co-workers [37] was not reached. After evaluation of the existing tools, ranging from commercial, to open source, and even considering cable solvers it was determined that a new solver had to be written.

\subsubsection{Chapter structure}

This chapter presents the most relevant details about the construction of the space/membrane equation software solver. The design constrains and requirements established at the beginning of the project are presented in the next section. After this, its base components are described. The design structure and an overview of the solver internals is presented in Section 6.4. In the last part of the chapter the description of how the software requirements were satisfied appears. A view of the capabilities of the tool with example solutions and resulting performance extends to the manuscript in Chapter 7 . A user guide and a programming manual for the tool appears in appendix Chapter B.

\subsection{Software requirements}

Pre-analysis of the functional requirements is one key step for the production of good quality computer software. A software requirement is a property the software must exhibit to solve a problem in the real world [124]. During the requirement analysis the software operational characteristics should be specified, its interfacing with other system components established, and the constrains the software must meet listed [125].

The core design objectives for the software tool were clear since the beginning of the project: to understand better the response of three-dimensional neurons and, to model these neurons in their respective three-dimensional conductive space. As a research project, the requirements were always oriented towards simulation of the more 
realistically possible electrical cell models, including the recent advances in knowledge about the electrical functioning of the neuron and neuronal stimulation. More specific requirements appeared after understanding better the physical problem and other requirements appeared after interaction with related tools (e.g. NEURON and bidomain solvers) and attempts to solve the problem with existing code (i.e. code used with collaborators at the beginning of the project $[38,89])$. General requirements such as good performance and ability to run in computer clusters architectures were obvious since the beginning. After two years of the project, and several failed attempts to use other tools the core requirements were set. Listed in order of importance these were:

1. Flexibility: The tool should be flexible enough to allow representing neuronal cells in different three-dimensional geometric configurations. Typical use cases were:

(a) Simulating the response of neurons under extracellular fields of varying spatial and temporal characteristics

(b) Simulation of the extracellular potentials produced by the cell's own activity

(c) Simulation of complex boundary conditions such as glass surfaces

(d) Heterogeneous conductivities

2. Flexible ion channel types: The tool should support more than the classic Hodgkin-Huxley channel model allowing tens of heterogeneously distributed channels such as those of Gold et al. [40]. Typical use cases were:

(a) Modeling of the axon initial segment

(b) Modeling of non-standard ionic gating schemes

3. Multiple cells: The tool should allow simulation of tens of cells in simultaneous activity. Typical use cases were:

(a) Filtering effects of other cell membranes in the extracellular potential signal

(b) Simulation of the generation of the local field potential

(c) Simulation of ephaptic interaction i.e. the interaction of cells via extracellular potentials only

4. Speed: Simulation of electro-physiological and neurological relevant events in the order of hundreds milliseconds to seconds had to be possible. Typical use cases were:

(a) Simulation of seconds long spike trains

(b) Simulation of synchronized activity between neurons

5. Geometrical expressiveness: The tool should allow representing neurons with process diameters in the sub-micron scale and even finer details. Use cases were: 
(a) Simulation of the thin end of dendrites

(b) Simulation of spines

(c) Simulation of the tight space between cells (around hundred nanometers)

6. Documentation: The tool and its source code should be properly documented and it should be usable by non programmer users. A user manual should be provided.

7. Parallelism: the tool must run in computer clusters and scale properly.

8. Open source: the tool should be based in free, open source libraries and formats for the representation of meshes, visualization and in general for its supporting infrastructure. The tool should also be open source and free itself.

These requirements guided the construction of the tool. Requirements 1 and 2 were the most relevant scientifically. The main goal of the tool was representing stimulation of cells but implementing the reduced set of Maxwell's equations allowed this. Around the middle of the project it was decided that no other tool in the market could provide these functionalities. Given the nature of the work, a doctoral thesis, it was decided that the the tool itself was going the be one of the main results.

Construction of a FEM tool is however not an easy programming task for a single programmer. Commercial and free FEM solvers are usually developed over many years and by tens of programmers. Careful design decisions had to be made for completion of the task. The use of open source tools was at the center of this (Requirement 8). Following the "don't reinvent the wheel" principles enough information had to be learned about the right base libraries. This will be presented in the next section.

Requirements 3 to 5 were more mathematically oriented. Enough had to be learned from FEM and in general numerical techniques to speed up the solution. This required a thorough review of the literature and existing methods and lead to the numerical improvements presented in Chapter 4. The availability of heart simulators provided a big boost in this area as at least a comparable system of equations existed. The specific details of the space/membrane equations however required great dedication and a large portion of the development time was spent on this. An open source heart tool (CHASTE) was used as the starting point for construction of the solver.

Requirement 6 can be considered standard in good software practices. A large portion of software tools developed in academic and scientific environments are simply "abandoned" after completion of the project. Proper documentation ensures other researchers can use it an extend it in the future ${ }^{3}$. Requirement 7 was fundamental for the solution of large system and is a must of any modern simulation software.

\subsection{Supporting infrastructure}

Finite element method solvers are large pieces of software that cannot possibly be built from scratch. The use of open source and free tools (requirement 8) was a key

\footnotetext{
${ }^{3} \mathrm{An}$ interesting discussion about this issue is presented by Pitt-Francis and co-workers [126].
} 
requirement given the limited budget of the project. Fortunately for the scientific community, a large number of good quality open tools exists for the construction of larger, research specific problem solvers. CHASTE-Membrane sits at the top of the open source environment CHASTE (Cancer, Heart and Soft-Tissue Environment [117]). The similarities of the CHASTE project with this thesis project were enough to consider it as the basic building block of the tool. As a heart electrophysiology solver, CHASTE already provided the ionic channel ordinary differential equation (ODE) solvers and the PDE solvers that are required for the space/membrane equations. Although these are connected in a different way, the main components of CHASTE were reusable enough. The representation of the membrane had to be implemented in CHASTE.

The software infrastructure in which CHASTE-Membrane sits is presented in the architectural Figure 6.1. This diagram presents from bottom to top the stack of main software components and the interfaces. To the sides, auxiliary tools that facilitate simulation and processing of results are also shown. The tools developed during this thesis are presented in light-red. CHASTE itself sits on top of a compendium of other open source libraries. The key components in this stack are:

- GMSH: GMSH (G-Mesh) is a three-dimensional finite element mesh generator. GMSH is used to convert a geometric CAD description in two actual meshes of triangles or tetrahedra. GMSH is not a standard CHASTE tool and a script was provided to convert GMSH output files to CHASTE files. See also figure 6.2 and appendix Chapter B.

- CellML: The Cell Modeling Language is a standard description language for storing and exchanging computer-based mathematical models. CellML grants access to a public library of ion channel kinetics defined in its standard structure.

- PETSc/MPI: PETSc (Portable, Extensible Toolkit for Scientific Computation) is a software library for the parallel solution of partial differential equations in the form of linear algebraic problems [103]. PETSc is based on MPI (Message Passing Interface) which supports the communication in parallel computers. PETSc supports the efficient representation of large matrices in sparse format.

- uBLAST/BOOST: uBLAST (micro Basic Linear Algebra Subprograms) is a $\mathrm{C}++$ class library for the representation and operations with dense or sparse matrices. In CHASTE, uBLAST is used for the handling of small matrices or vectors (typically four elements). BOOST is a set of general $\mathrm{C}++$ libraries that provide utilities such as configuration management.

- XSD/Xerces: Libraries for the parsing of eXtended Markup Language (XML) files and XML Schema files. This is used by CHASTE for reading of configuration files.

- Tetgen/Triangle: Libraries for the processing of mesh description files in the Tetgen (3D) and Triangle (2D) formats.

- Paraview/VTK: Paraview is the main tool used for visualization of the results produced by CHASTE and CHASTE-Membrane. VTK (Visualization Toolkit) is 


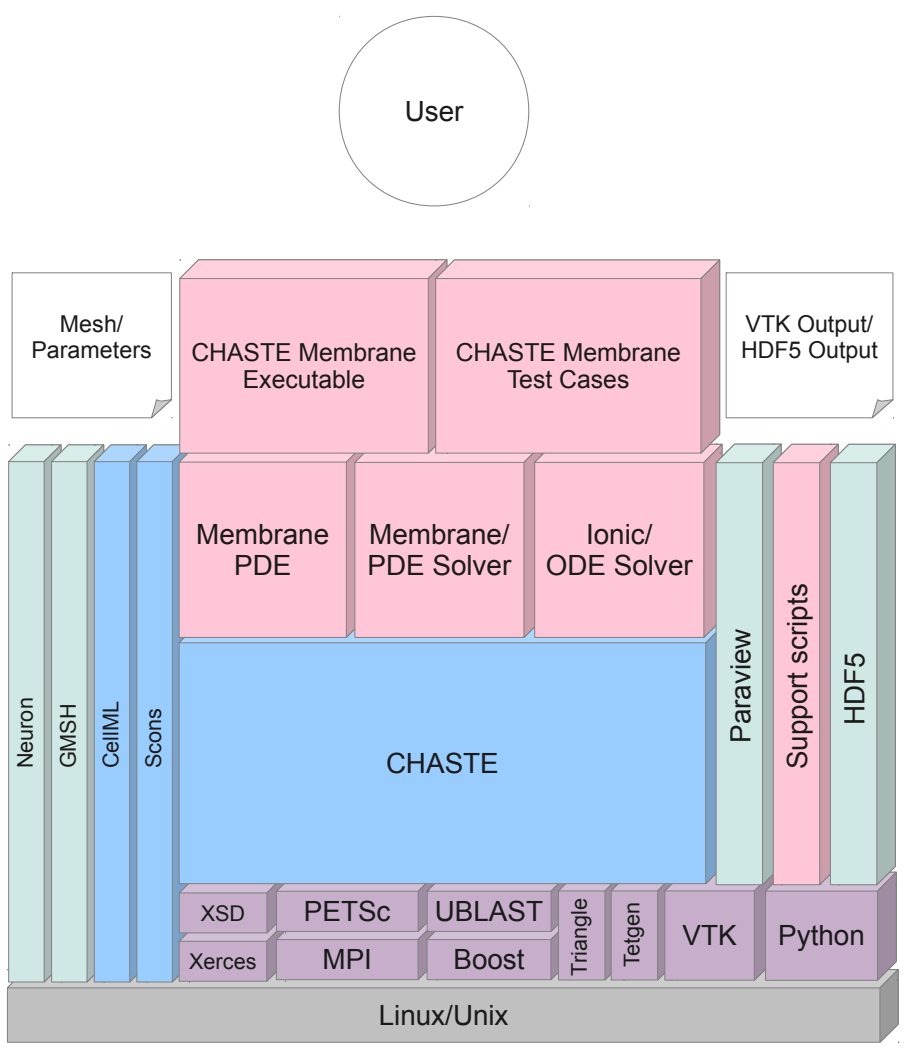

Figure 6.1: The software architecture of CHASTE-Membrane. The tools developed during this thesis are presented in light-red.

a set of libraries for the management of visualization meshes with multiple floating point and vector values associated to their nodes. VTK is at the core of Paraview.

- HDF5: HDF5 (Hierarchical Data Format version 5) is a toolbox and file format for the management of extremely large and complex data collections. HDF5 is the format in which CHASTE and CHASTE-Membrane data output is stored. HDF5 guarantees efficiency on the accessing of this data.

CHASTE itself provides documentation about its connection to these components and the compiling toolbox Scons is described elsewhere ${ }^{4}$. NEURON and Python are described elsewhere [115, 127].

\subsection{Software design}

For the design of the software standard UML (Unified Modeling Language, [128]) diagrams were used. The use of UML in the context of the CHASTE environment allowed to visualize the relation between components and correctly interface the new functionalities offered by CHASTE-Membrane. These design diagrams are provided for future

\footnotetext{
${ }^{4}$ http://www.scons.org/
} 
reference in the appendix Chapter D. Diagrams A.1 and A.2 represent the object oriented inheritance relations of the objects constructed in CHASTE-Membrane with the core objects of CHASTE. The solving sequence, that is, the way in which objects intercommunicate during the solving procedure are presented in the appendix diagrams A.3 to A.5. These diagrams were fundamental for the development of the $\mathrm{C}++$ implementation. The UML diagrams for CHASTE are available in its documentation ${ }^{5}$.

\subsection{Results and performance}

CHASTE-Membrane version 1.0 was developed during 21 months. Version 1.0 was considered a mature software ready to be used by others and with the required supporting information. The tool has been already offered to other developers in the community. In its current form some of the tested features the tool offers are:

- Representation of a few cells with shapes from simple real cell reconstructions such as in Figure 1.3

- Stimulation with homogeneous and point source fields

- Heterogeneous ion channels and passive properties for every mesh triangle defining the membrane

- Mixed boundary conditions (time dependent Dirichlet or Neumann)

- Anisotropic conductivities in the intra- and extracellular space

- Other neurons and various types of cells can be added (e.g. glia) anywhere in the extracellular volume space

- Minimum mesh elements of approximately $200 \mathrm{~nm}$

The manuscript in Chapter 7 presents these features at work. Most of the software requirements established in the design phase have been satisfied. Satisfaction of Requirement 1 and requirement 2 are shown in action in Section 3.5 of Chapter 7. Completion of Requirement 3 is shown in Section 3.4 of Chapter 7. Completion of Requirement 4 is shown in Section 3.1-3.2 of Chapter 7. Compliance of Requirement 5 is shown in Section 3.2-3.5 of the same chapter. Documentation (Requirement 6) is provided by this thesis work and the document on appendix Chapter B. A typical simulation with the CHASTE-Membrane tool follows the procedures shown in the pipeline of Figure 6.2. The tool is open source and publicly available agreeing with Requirement 8. Parallel execution (Requirement 7) is supported and the simulation in Section 3.4 and 3.5 of Chapter 7 were run in multi-core computers. Still better mesh partitions are required. This will be discussed in Chapter 8 .

\footnotetext{
${ }^{5}$ http://www.cs.ox.ac.uk/chaste/documentation.html
} 


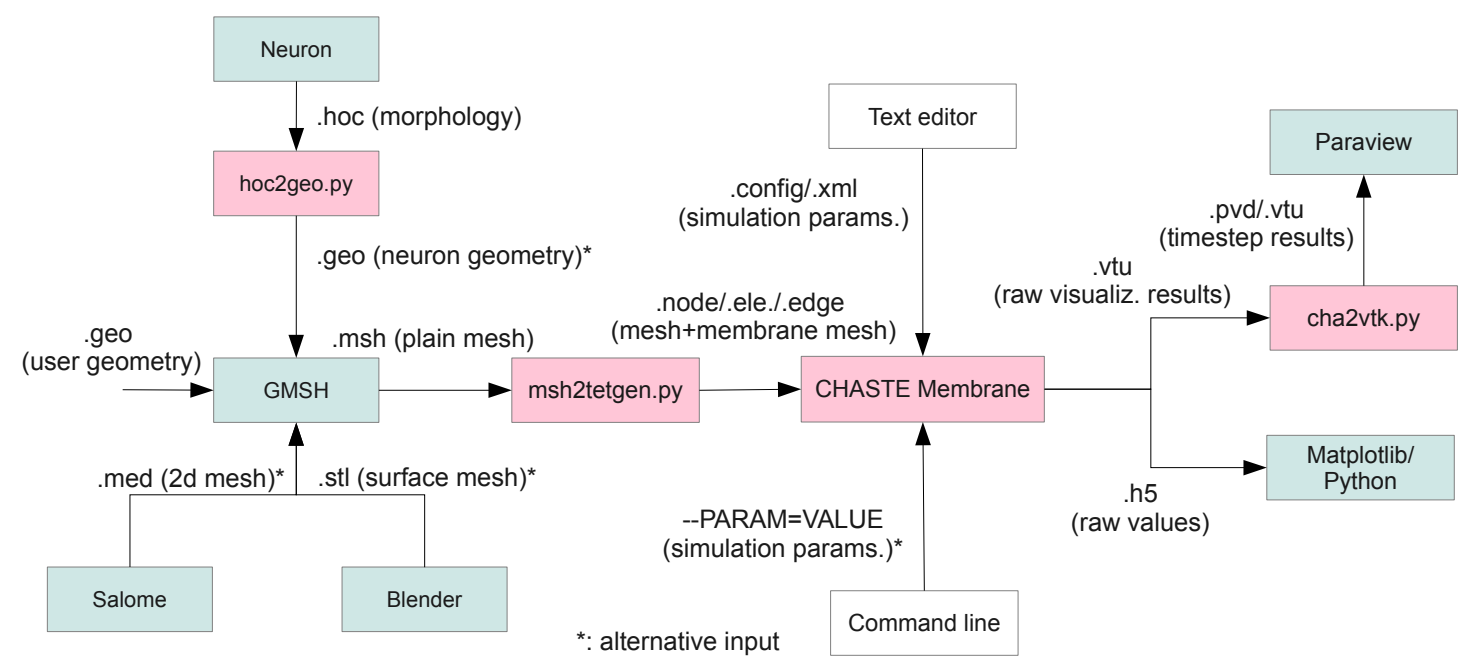

Figure 6.2: A typical simulation pipeline. Flow is described in Chapter B.

\subsection{Summary}

In this chapter the implementation of the space/membrane equations was presented. This set of equations required a specialized tool that was not available in the free or commercial software market. Finite element method solvers are not simple programs and their implementation should not be taken lightly. The tool had to satisfy a number of design requirements that guided its development. The use of open source tools and the release of the software solver itself as an open package was beneficial for development and will hopefully be so for the neuroscience community. Modern software design techniques such as UML were used in the development process. The heart bidomain solver CHASTE was used as the base sub-component of the solver. CHASTE provided many functionalities that were required in the solution of the main set of equations but others were missing and had to be implemented. The core software requirements were met. The execution of simulations with CHASTE-Membrane has good performance but improvements are possible as it will be discussed in Chapter 8 . 


\section{Fast simulation of electrical activity at cell membranes, interacting with self-generated and externally imposed electric fields}

The following chapter consists of a manuscript submitted on July 23, 2012 to the IOP Journal of Neural Engineering ${ }^{1}$. This manuscript represents a results chapter in the overall thesis work. The manuscript was submitted under an Open Access policy that grants their authors and their institutions to reproduce the article $^{2}$. The manuscript compares the method proposed to previous approaches (e.g. the cable equation). All the text and tables in the manuscript were written by Andres Agudelo-Toro (AAT) and revised by Andreas Neef (AN). All the numerical experiments on the article were performed by AAT. All the Figures of the article were created by AAT and revised by $\mathrm{AN}$.

\footnotetext{
${ }^{1}$ By the time this thesis document was revised (November 2013) a more refined version of this manuscript has been accepted and published by the same journal in the April 2013 edition as: A. Agudelo-Toro, A. Neef: "Computationally efficient simulation of electrical activity at cell membranes interacting with self-generated and externally imposed electric fields". J. Neural Eng. 10 026019, 2013. The draft of the manuscript presented in this dissertation in October 2012 has been preserved.

${ }^{2}$ http://iopscience.iop.org/info/page/openaccess
} 


\title{
Fast Simulation of Electrical Activity at Cell Membranes, Interacting with Self-generated and Externally Imposed Electric Fields
}

\author{
Andres Agudelo-Toro ${ }^{1,2}$ and Andreas Neef 1,2,3
}

July 20, 2012

\begin{abstract}
${ }^{1}$ Max Planck Institute for Dynamics and Self-Organization, Göttingen, Germany. ${ }^{2}$ Bernstein Focus Neurotechnology, Göttingen, Germany. ${ }^{3}$ University Medicine Göttingen, Germany.
\end{abstract}

\section{Abstract}

The electric activity of neurons creates extracellular potentials. Recent findings show that these endogenous fields act back onto the neurons, contributing to synchronization of population activity. The influence of this endogenous fields is also relevant for understanding therapeutic approaches such as transcranial direct current stimulation, transcranial magnetic stimulation and deep brain stimulation. The mutual interaction between fields and membrane currents is not captured by today's concepts of cellular electrophysiology, as those concepts are based on isolated membranes in an infinite, isopotential extracellular space. Even the direct influence of fields is not correctly represented by the commonly used activating function. While a reduced set of Maxwell's equations can be used to couple membrane currents to extraand intracellular potentials, this approach is rarely taken, most likely because adequate computational tools are missing. We present a computational method that implements this set of equations. It allows simulation of cells under realistic conditions: sub-micron cell morphology, various ion channel properties and distributions and a conductive, nonhomogeneous space. By introducing an implicit solver, numerical stability is attained even with large time-steps that are limited only by the time development of the membrane potentials. This allows simulation times of tens of minutes instead of weeks, even for complex problems. The extracellular fields are accurately represented, including secondary fields, which originate at inhomogeneities of the extracellular space and can reach several millivolts. We present a set of instructive examples that show how this method can be used to obtain reference solutions for problems, which might not be accurately captured by the traditional methods.

\section{Introduction}

Extracellular potentials (EP) influence the activity of neurons. Neuronal activity itself, synaptic currents, subthreshold oscillations and action currents sum-up over neuronal populations and continuously change local potentials. Recent findings contribute to the mounting evidence that these endogenous EPs talk back to neurons and influence synchronization of firing patterns in vitro and in vivo $[1,2,3]$. Moreover, in therapy and diagnostics, strong, super-threshold fields are used in the form of electroconvulsive therapy, transcranial magnetic stimulation or deep brain stimulation to activate large populations of neurons $[4,5,6]$. Recently, techniques that utilize weaker, sub-threshold fields such as direct current stimulation or alternating current stimulation have been developed $[7,8]$. These were reported to influence motor cortex excitability and higher functions like motor learning or memory $[9,10,11]$.

Although a number of modeling tools have been introduced to study the interaction of EPs and neurons $[12,13,14,15,16,17]$, new research tools are required to more accurately model this relation. In some applications it is enough to calculate the EP waveform from a given number of neuronal sources [18], or to simply study the unidirectional effects of EPs $[19,20,21]$. However, for key applications such as understanding of how local potentials facilitate synchronization of cell ensembles, or to model simultaneous stimulation and recording in complex geometrical set-ups (e.g. multi unit arrays and novel brain-machine interfaces $[22,23,24,25])$, it is important to understand both directions of the neuron-EP interplay [26, 17].

When the "forward" effect, i.e. the creation of EPs by neuronal activity, is of interest, EPs are calculated with the line-source approximation [14]: the membrane currents are computed for each linear segment of a one-dimensional compartmental neuron model. These currents are then used to calculate the EP according to standard volume conductor theory $[26,27,28]$. This approach does not consider the feed-back from EPs in the neuron and its applicability is limited to locations farther than $1 \mu \mathrm{m}$ away from the active membrane [14]. Interactions between adjacent cells within sub-micrometer distance, and the effect of clustering of ion channels cannot be treated. Furthermore, volume conductor theory assumes the extracellular medium to be homogeneous and isotropic. This ignores the strong secondary fields caused by the inhomogeneities of tissue [29], which give rise to the so called "virtual electrodes", and that can dominate the effects of EPs on excitable tissue [30].

To simulate the "feed-back" effect, i.e. the changes in the cell caused by EPs, similar principles are connected in a different sequence. The EPs are computed from external current sources while assuming homogeneity and isotropy, hence ignoring the possibility of virtual electrodes. Neurons are then represented by a concatenation of one-dimensional cables and the effect of the EP is included by means of the activating function [12] as an additional source term in the cable equation [31]. In finite cables, this effect is calculated by projecting the extracellular field on to the axis of the cables, and the stimulation itself is emulated by current injection in the ends $[32,33,34,35]$.

The activating function is an approximation whose utility 
has been questioned [36, 37]. As it simply acts as an additional source term in the cable equation, its applicability is also limited to particular geometries. A simple illustration of the problem is its inability to properly represent a spherical cell body inside a homogeneous field. To compute the effect, the sphere has to be approximated by cylindrical sections. When the field is directed perpendicular to the cylinders' axes, this does not exert any influence on the cell body (see Fig. 5). The simulated effect of the EP on this symmetric structure erroneously depends on the cosine of the polar angle of the field direction.

A correct solution of the forward and feed-back problem in the neuron-EP interaction requires a complete spatial representation of the neuronal membrane and its relation to the intra and extra-cellular potentials. This comprises a selfconsistent solution of the Laplace equation governing the potential, and the non-linear equations that determine the voltage-dependent membrane currents (i.e. the sources of the potential changes). The solution to the Laplace equation for arbitrary geometries can be achieved with finite difference (FDM), boundary element (BEM), finite volume (FVM), or finite element methods (FEM). A numerical time iteration scheme then has to be selected to model the evolution of the potentials.

FEM and related methods have been used to model stimulation of arbitrarily shaped cells for studies of the effects of electroporation, as well as to model simple neuronal geometries [13, 15, 38, 17, 39]. These approaches have however failed to provide a widely available tool that can fully model the neuron-EP bidirectional interaction. The deficiencies can be summarized in three aspects: computational limitations to represent detailed geometries, lack of efficient time evolution schemes, and the use of closed and commercial numerical solvers. FDM approaches such as that described in [38] represent geometries in meshes with a fixed-grid spacing determined by the smallest feature in the domain. Due to the very large number of elements, the computation time of these simulations is impractically long for realistic, sub-micrometer morphological features. BEM approaches [40, 41], although more computationally efficient, cannot represent spatially heterogeneous conductivities. FEM and FVM have been employed in other cases $[13,15,16,17,42,39]$, but these systems have been limited to unrealistic spatial scales $[16,42]$, restrictive time evolution schemes [13, 15, 16, 42, 39], two dimensions [15], and the absence of ion channels $[13,15,16,39]$. The use of closed source $[15,16,42]$ and commercial software $[13,17,39]$ is a common feature among all these works, hindering its applicability and replicability in other scientific studies.

In this work, we present a numerical method for the treatment of heterogeneous, three-dimensional, intra- and extracellular spaces separated by membranes with voltagedependent conductances. To permit efficient simulation of detailed geometries, two main strategies are used: first, the equations governing the time evolution of the electric potential in space were separated from the non-linear equations that treat the membrane currents. This follows the ideas of heart bidomain solvers [43] and a previous approach [15]. Second, an implicit time iteration scheme based on the Crank-Nicolson (CN) method is introduced. This scheme allows simulation time-steps orders of magnitude larger than the Euler scheme, without causing numerical instabilities. The use of this implicit method also grants applicability of adaptive time step schemes, which can further shorten computation times by one to two orders of magnitude.It enables simulations that run over physiologically relevant times (tens to hundreds of milliseconds), represent the detailed, submicrometer cell morphology and include ion channels with arbitrary voltage-dependent gating schemes.

The importance of computationally efficient methods is emphasized if the details of neuronal geometries are considered, e.g. in fine distal dendrites, or the sub-micrometer distances between cells. In the $2 \mathrm{D}$ simulations of Ying and Henriquez [15] for instance, the explicit Euler method was used. The explicit Euler method requires extremely fine time steps for micrometer geometries, limiting the physiological times that can be simulated. These requirements become more stringent as the geometry is extended to $3 \mathrm{D}$, and the complexity of the spatial domain increases. Besides the realistic, true sub-micron distances fundamental for the study of cell-to-cell interaction, times beyond a few milliseconds are crucial for the study of neuronal synchronization and more complex stimulation protocols. To our knowledge, such simulations have not been performed, most likely because of the large computation time involved.

The finite element method presented here has been implemented in an open source software we named CHASTEMembrane. It can represent stimulation, recording and feedback between extracellular fields and membrane voltage at realistic spatial and temporal scales. In the next sections we introduce the physical model and provide some essential details of the solving procedure. Treating standard geometries, we demonstrate that the numerical solutions with the method converge to the analytical solutions. We present stability conditions for the numerical scheme chosen, describing the time step limits for a given spatial discretization. Finally, we point out that the presented approach can treat problems that are only inadequately captured by the cable equation, activating function and line source approximations. This suggests a use of CHASTE-Membrane as a reference to test and correct the solutions obtained by the widely used cable equation solvers such as NEURON and GENESIS. By working through a set of instructive examples, we demonstrate the importance of simulating neurons with realistic time and space scales. The toolbox is available under the project CHASTE [44] (http://www.cs.ox.ac.uk/chaste).

\section{Methods}

\subsection{Description of the model}

The model represents the electrical activity of a biological cell inside a conductive medium (Fig. 1(A)). Space is segregated into an extracellular domain $\Omega_{e}$ and possibly multiple intracellular domains $\Omega_{i}\left(\Omega=\Omega_{e} \cup \Omega_{i}\right)$. The domains do not overlap $\left(\Omega_{e} \cap \Omega_{i}=\emptyset\right)$ and are each characterized by space dependent conductivity tensors $\sigma_{e}$ and $\sigma_{i}$. Unit normal vectors at domain boundaries point outward and are denoted $\mathbf{n}_{e, i}$. Intra- and extracellular domains are separated by membranes $\Gamma$, assumed of zero thickness. Membranes accumulate charge due to capacitance, and can harbor voltage dependent ion channels. The voltage channels can follow non-linear differential equations, for instance HodgkinHuxley-like kinetics.

For neural electric phenomena, a few simplifications in 


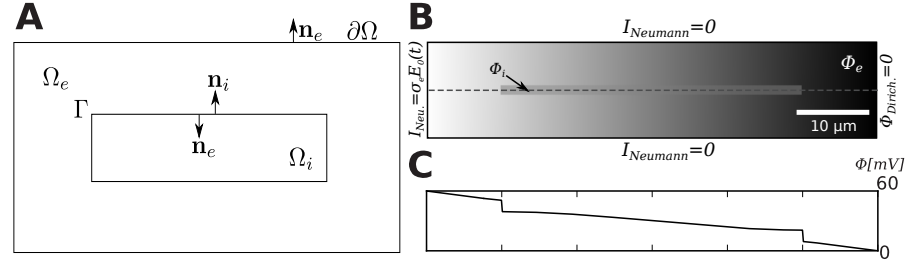

Figure 1: Schematic of the solution domain (SD) and example boundary conditions (BC). (A) The SD is divided in intra $\left(\Omega_{i}\right)$ and extracellular $\left(\Omega_{e}\right)$ regions, separated by a membrane $(\Gamma)$. The exterior boundary is represented by $\partial \Omega$ and unit boundary normals for both domains are represented by $\mathbf{n}_{e, i}$. (Note the extracellular boundary normal points to the interior of the cell). (B) Example of a 2D model with mixed boundary conditions (BC). The model and simulation tool allow time dependent mixed Neumann and Dirichlet conditions. In this case, Neumann zero flux $\mathrm{BC}$ are used at the top and bottom boundaries, while a Dirichlet zero BC used on the right represents a ground electrode. Stimulation can be driven by a time dependent current density on the left boundary. An elongated cell is present in the center of the domain. The flux and ground create extracellular $\left(\Phi_{e}\right)$ and intracellular $\left(\Phi_{i}\right)$ potential gradients. (C) Corresponding potential for the stimulus gradient along the dashed line in (B) after $2 \mu$ s of stimulation $\left(|\mathbf{E}|=10^{4}\right.$ $\left.\mathrm{mV} / \mathrm{cm}, \sigma_{e}=\sigma_{i}=10 \mathrm{mS} / \mathrm{cm}\right)$.

Maxwell's equations are possible. Typical spatial and temporal dimensions of neural current fluxes, together with the dielectric and magnetic parameters of biological media render the feedback from the induced magnetic field onto the electric field negligible. Electromagnetic waves do not play an important role in the scales observed. Water is highly polarizable, and this combined with the typical conductance of biological tissue, imply that any free, unbalanced charge is balanced within fractions of a nanosecond. This is faster than the scale at which the electric processes of interest unfold.

These two relations justify the use of the quasi-static approximation of the Maxwell's equations. In this approximation the charge density in aqueous media is assumed to be zero $[45,46]$. This however does not exclude the possibility of current volume density sources, and they are allowed in the extra- and intra-cellular space $\left(\rho_{e, i}\right)$. In the quasi-static approximation, the Poisson's equation governs the extraand intra-cellular potentials $\Phi_{e, i}$ :

$$
\begin{aligned}
& -\nabla \cdot \sigma_{e}(x) \nabla \Phi_{e}(x, t)=\rho_{e}(x, t) \text { in } \Omega_{e} \\
& -\nabla \cdot \sigma_{i}(x) \nabla \Phi_{i}(x, t)=\rho_{i}(x, t) \text { in } \Omega_{i} .
\end{aligned}
$$

In the absence of current sources, this simplifies to Laplace's equation,

$$
-\nabla \cdot \sigma_{e} \nabla \Phi_{e}=-\nabla \cdot \sigma_{i} \nabla \Phi_{i}=0 .
$$

On the exterior boundary $\partial \Omega_{e}$, Dirichlet, Neumann or a mixture of both boundary conditions (BC) can be applied (Fig. 1(B,C)):

$$
\begin{gathered}
\Phi_{e}=\Phi_{D}(x, t) \text { on } \partial \Omega_{D} \\
\sigma_{e} \nabla \Phi_{e} \cdot \mathbf{n}_{e}=I_{N}(x, t) \text { on } \partial \Omega_{N} .
\end{gathered}
$$

If a grounding Dirichlet boundary is specified the solution is unique. When only Neumann BCs are specified, the problem is solvable up to an arbitrary constant if the total current through the boundary is conserved.

Membrane voltage is defined for any given point on the membrane as the potential difference for the same point in intra- and extracellular space:

$$
V_{m}=\Phi_{i}(x)-\Phi_{e}(x) \text { on } \Gamma .
$$

Extracellular and intracellular current towards $\Gamma$ is continuous for any small volume intersecting it, and is denoted by the membrane current $I_{m}$ :

$$
I_{m}=\mathbf{n}_{e} \cdot \sigma_{e} \nabla \Phi_{e}=-\mathbf{n}_{i} \cdot \sigma_{i} \nabla \Phi_{i} \text { on } \Gamma .
$$

As current reaches one patch of membrane, it can either trigger the same amount of current being released in the opposite side, or cross it through ionic flux. Total membrane current corresponds to the exchange of capacitive current plus the sum of transmembrane ionic currents $\left(I_{i o n}\right)$ :

$$
I_{m}=C_{m} \frac{\partial V_{m}}{\partial t}+I_{i o n}
$$

with $C_{m}$ the membrane capacitance per unit area. Ionic currents $I_{i o n}\left(V_{m}, \mathbf{u}\right)$ can be determined by non-linear ordinary differential equations depending on the membrane voltage and a vector of gating variables $\mathbf{u}_{\boldsymbol{\Gamma}}$ at every node of $\Gamma$ :

$$
\frac{\partial \mathbf{u}}{\partial t}=\mathbf{f}\left(V_{m}, \mathbf{u}_{\boldsymbol{\Gamma}}\right)
$$

A simple passive membrane current can also is used:

$$
I_{\text {ion }}=\frac{V_{m}}{R_{m}}
$$

with $R_{m}$ a constant membrane resistance. Unless specified, most of the simulations in the results section will use this type of current. The main variables and parameters of the model are summarized in the appendix Table S.1.

\subsection{Space discretization}

To solve the complete space and membrane problem, the equations governing the potentials in the intra- and extracellular domains and the equations governing the time development of the membrane currents are solved alternatingly. Eqs. (1), (2), and (6) with BC (3) and (4) are solved for a fixed moment in time with a fixed $V_{m}$ using the FEM. A complete discretization and proof of existence of the solution for this spatial problem was presented by Lamichhane and Wohlmuth [47]. A brief summary of the FEM discretization is shown next.

At any given moment, the membrane voltage $V_{m}$ is assumed fixed and the system of partial differential equations is solved for variables $I_{m}$ and $\Phi_{e, i}$ ( $\Phi$ when the domain is indifferent). The classic weak FEM formulation is obtained by multiplying equations (1) and (2) by test functions $v_{e}, v_{i}$, integrating and applying Green's first identity:

$$
\int_{\Omega_{e}} \sigma_{e} \nabla \Phi_{e} \cdot \nabla v_{e} d \mathbf{x}-\int_{\Gamma} I_{m} v_{e} d s=\int_{\partial \Omega_{N}} I_{N} v_{e} d s+\int_{\Omega_{e}} \rho_{e} v_{e} d \mathbf{x}
$$

$$
\int_{\Omega_{i}} \sigma_{i} \nabla \Phi_{i} \cdot \nabla v_{i} d \mathbf{x}+\int_{\Gamma} I_{m} v_{i} d s=\int_{\Omega_{i}} \rho_{i} v_{i} d \mathbf{x} .
$$

In Eq. (9) the assumption that no cell is open to the exterior boundary is made. The interface condition on $\Gamma$ is comparable to a Neumann condition, with the difference that in this model, $I_{m}$ is an unknown, sub-dimensional function on membrane space. The condition (5) is expressed in the weak form as,

$$
\int_{\Gamma}\left(\Phi_{i}-\Phi_{e}\right) u d s=\int_{\Gamma} V_{m} u d s
$$


with $u$ a test function also restricted to membrane space. Eq. (10) can be understood as a special Dirichlet BC where the values must comply with a fixed potential difference $V_{m}$. The complete system of spatial equations is formed by (10) and the sum of Eqs. (8) and (9):

$$
\int_{\Omega} \sigma \nabla \Phi \cdot \nabla v d \mathbf{x}+\int_{\Gamma} I_{m}[v]_{e, i} d s=\int_{\partial \Omega_{N}} I_{N} v d s+\int_{\Omega} \rho v d \mathbf{x} .
$$

Here, $\sigma, \Phi, v$ represent the corresponding symbol in any of the non overlapping domains $\Omega_{e, i}$. In (11) the operator [ $]_{i, e}$ changes the sign of the operand according to the face $\Gamma$ that is being evaluated (integration is performed in the intracellular and extracellular face of $\Gamma$ ).

The matrix form of the problem is obtained by choosing a set of piecewise linear basis functions $\left\{v^{1}, v^{2}, \ldots, v^{N_{\Omega}}\right\}$ for each of the $N_{\Omega}$ nodes in a triangulation $\Omega^{h}$, and $\left\{u^{1}, u^{2}, \ldots, u^{N_{\Gamma}}\right\}$ for each of the $N_{\Gamma}$ nodes in a triangulation $\Gamma^{h}$. The result is a system of $M=N_{\Omega}+N_{\Gamma}$ equations,

$$
\int_{\Omega} \sigma \nabla \Phi \cdot \nabla v^{\mathrm{i}} d \mathbf{x}+\int_{\Gamma} I_{m}\left[v^{\mathrm{i}}\right]_{e, i} d s=\int_{\Omega} \rho v^{\mathrm{i}} d \mathbf{x} \quad \mathrm{i}=1, . ., N_{\Omega},
$$

$$
\int_{\Gamma} u^{\mathrm{j}}[\Phi]_{e, i} d s=\int_{\Gamma} u^{\mathrm{j}} V_{m} d s \quad \mathrm{j}=1, . ., N_{\Gamma} \text {. }
$$

The Neumann term $\int_{\partial \Omega_{N}} I_{N} v d s$ was dropped but can be added in any future step.

Let be $\Phi^{\mathrm{k}}, I_{m}^{\mathrm{j}}$, and $V_{m}^{l}$ the approximations of values $\Phi\left(x^{\mathrm{k}}\right), I_{m}\left(x^{\mathrm{j}}\right)$ and $V_{m}\left(x^{l}\right)$ at triangulation nodes $x^{\mathrm{k}} \in \Omega^{h}$, $x^{\mathrm{j}} \in \Gamma^{h}$ and $x^{\mathrm{l}} \in \Gamma^{h}$. The continuous functions can then replaced by $\Phi \approx \sum_{\mathrm{k}=1}^{N_{\Omega}} \Phi^{\mathrm{k}} v^{\mathrm{k}}, I_{m} \approx \sum_{\mathrm{j}=1}^{N_{\Gamma}} I_{m}^{\mathrm{j}} u^{\mathrm{j}}$, and $V_{m} \approx \sum_{\mathrm{l}=1}^{N_{\Gamma}} V_{m}^{\mathrm{l}} u^{\mathrm{l}}$, and the gradient by $\nabla \Phi \approx \sum_{\mathrm{k}=1}^{N_{\Omega}} \Phi^{\mathrm{k}} \nabla v^{\mathrm{k}}$.

Equations (12), (13) for a given $\mathrm{i}$ and a given $\mathrm{j}$ are now:

$$
\sigma \sum_{\mathrm{k}} \Phi^{\mathrm{k}} \int_{\Omega} \nabla v^{\mathrm{k}} \cdot \nabla v^{\mathrm{i}} d \mathbf{x}+\sum_{\mathrm{j}} I_{m}^{\mathrm{j}} \int_{\Gamma} u^{\mathrm{j}}\left[v^{\mathrm{i}}\right]_{i, e} d s=\int_{\Omega} \rho v^{\mathrm{i}} d \mathbf{x},
$$

$$
\sum_{\mathrm{k}} \Phi^{\mathrm{k}} \int_{\Gamma} u^{\mathrm{j}}\left[v^{\mathrm{k}}\right]_{i, e} d s=\sum_{\mathrm{l}} V_{m}^{\mathrm{l}} \int_{\Gamma} u^{\mathrm{l}} u^{\mathrm{j}} d s
$$

Left hand entries of the matrix linear system form can be defined then as $A^{\mathrm{ik}}=\sigma \int_{\Omega} \nabla v^{\mathrm{k}} \cdot \nabla v^{\mathrm{i}} d \mathbf{x}$ and $B^{\mathrm{ij}}=$ $\int_{\Gamma} u^{\mathrm{j}}\left[v^{\mathrm{i}}\right]_{i, e} d s$. Right hand vector entries can be defined as $f^{\mathrm{i}}=\int_{\Omega} \rho v^{\mathrm{i}} d \mathbf{x}$ and $G^{\mathrm{j} l}=\int_{\Gamma} u^{\mathrm{l}} u^{\mathrm{j}} d s$. The system of equations,

$$
\begin{aligned}
\sum_{\mathrm{k}} \Phi^{\mathrm{k}} A^{\mathrm{ik}}+\sum_{\mathrm{j}} I_{m}^{\mathrm{l}} B^{\mathrm{ij}} & =f^{\mathrm{i}} \\
\sum_{\mathrm{k}} \Phi^{\mathrm{k}} B^{\mathrm{jk}} & =\sum_{\mathrm{l}} V_{m}^{\mathrm{l}} G^{\mathrm{j} \mathrm{l}},
\end{aligned}
$$

can be written in matrix form as,

$$
\mathbf{M u}=\left[\begin{array}{cc}
\mathbf{A} & \mathbf{B} \\
\mathbf{B}^{T} & \mathbf{0}
\end{array}\right]\left[\begin{array}{c}
\mathbf{\Phi} \\
\mathbf{I}_{m}
\end{array}\right]=\left[\begin{array}{c}
\mathbf{f} \\
\mathbf{G} \mathbf{V}_{m}
\end{array}\right]=\mathbf{b}
$$

Matrix equation (18) has to be solved to obtain the potential $\Phi$ and membrane current $\mathbf{I}_{m}$ at any time step.
Although the Laplacian A matrix is positive definite, in general the matrix $\mathbf{M}$ is symmetric but indefinite. In [47] a factorization procedure is presented which can produce an equivalent positive definite system after elimination of vector $\mathbf{I}_{m}$ through static condensation. In that form, the Conjugate Gradient (CG) method can be used to solve the linear system. This form was used for the explicit Euler solver presented in the next sections. Although the details of the factorization in [47] will not be presented in this paper, we will still motivate the expression for $\mathbf{I}_{m}$, as it is required for stability analysis. This expression can be readily extracted from Eq. (18),

$$
\mathbf{B I}_{m}=\mathbf{f}-\mathbf{A} \boldsymbol{\Phi} .
$$

Due to symmetry between the membrane current expression and the voltage potential expressions (note that the $I_{m}$ part of (16) and the $\Phi$ part of (17) produce the same matrix B), and a special node re-organization (nodes that touch the interface are moved to the bottom of the matrix), a left pseudo-inverse matrix $\mathbf{W}^{T}$ can be constructed for $\mathbf{B}$ such that,

$$
\mathbf{I}_{m}=\mathbf{W}^{T}(\mathbf{f}-\mathbf{A} \boldsymbol{\Phi})
$$

This expression depends only on $\boldsymbol{\Phi}$. Replaced back into Eq. (18), a linear system exclusively in terms of $\boldsymbol{\Phi}$ can be obtained.

\subsection{Time discretization}

Equation (7) is solved for a discrete time step after solution of Eqs. (1), (2), and (6) with the procedure specified in section 2.2. At each time step, the discrete space equations (11), (10) are solved for a fixed voltage vector $\mathbf{V}_{m}$. The resulting $\mathbf{I}_{m}$ is used together with the vector of ionic currents per node $\mathbf{I}_{i o n}\left(\mathbf{V}_{m}^{\mathrm{n}}\right)$ to find the next membrane voltage. The time iteration can be either explicit with the Euler method or in a implicit Crank-Nicolson (CN). The explicit scheme is easily obtainable with the previous values:

$$
\mathbf{V}_{m}^{\mathrm{n}+1}=\mathbf{V}_{m}^{\mathrm{n}}+\frac{\Delta t}{C_{m}}\left(\mathbf{I}_{m}^{\mathrm{n}}-\mathbf{I}_{i o n}^{\mathrm{n}}\right)
$$

The new $\mathbf{V}_{m}^{n+1}$ is then ready to be used again to solve the spatial equations.

The Euler scheme is however limited (see section 3) and an implicit $\mathrm{CN}$ was implemented. The $\mathrm{CN}$ scheme requires the next membrane current value $\mathbf{I}_{m}^{\mathrm{n}+1}$ :

$$
\mathbf{V}_{m}^{\mathrm{n}+1}=\mathbf{V}_{m}^{\mathrm{n}}+\frac{\Delta t}{C_{m}}\left(\frac{1}{2}\left(\mathbf{I}_{m}^{\mathrm{n}+1}+\mathbf{I}_{m}^{\mathrm{n}}\right)-\mathbf{I}_{i o n}^{\mathrm{n}}\right)
$$

$\mathbf{I}_{m}^{\mathrm{n}+1}$ is an unknown future value of $\mathbf{I}_{m}$ an might prompt for an additional numerical strategy (e.g. a Newton solver). Still, through careful re-arrangement of the terms the same linear solver used to find $\boldsymbol{\Phi}$ can be employed. Defining $c=$ $\frac{\Delta t}{2 C_{m}}$ and $\mathbf{g}^{\mathrm{n}}=\mathbf{V}_{m}^{\mathrm{n}}+\frac{\Delta t}{C_{m}}\left(\frac{1}{2} \mathbf{I}_{m}^{\mathrm{n}}-\mathbf{I}_{i o n}^{\mathrm{n}}\right)$ one obtains:

$$
\mathbf{V}_{m}^{\mathrm{n}+1}=c \mathbf{I}_{m}^{\mathrm{n}+1}+\mathbf{g}^{\mathrm{n}} .
$$

Combining the time iteration with the linear system as a vector in the advanced time step $\mathbf{M} \mathbf{u}^{\mathrm{k}+1}=\mathbf{b}^{\mathrm{k}+1}$ results in:

$$
\left[\begin{array}{cc}
\mathbf{A} & \mathbf{B} \\
\mathbf{B}^{T} & \mathbf{0}
\end{array}\right]\left[\begin{array}{l}
\mathbf{\Phi}^{\mathrm{n}+1} \\
\mathbf{I}_{m}^{\mathrm{n}+1}
\end{array}\right]=\left[\begin{array}{c}
\mathbf{f} \\
\mathbf{G}\left(c \mathbf{I}_{m}^{\mathrm{n}+1}+\mathbf{g}^{\mathrm{n}}\right)
\end{array}\right] .
$$


After matrix manipulation and $\mathbf{C}=-c \mathbf{G}$ the new linear system is,

$$
\left[\begin{array}{cc}
\mathbf{A} & \mathbf{B} \\
\mathbf{B}^{T} & \mathbf{C}
\end{array}\right]\left[\begin{array}{l}
\mathbf{\Phi}^{\mathrm{n}+1} \\
\mathbf{I}_{m}^{\mathrm{n}+1}
\end{array}\right]=\left[\begin{array}{c}
\mathbf{f} \\
\mathbf{G g}^{\mathrm{n}}
\end{array}\right] .
$$

For solution of (22), the system was factorized to eliminate $\mathbf{I}_{m}$ as was done with system (18). However after the same factorization procedure, [47] the left hand side is nonsymmetric (due to matrix $\mathbf{C}$ ). The Generalized Minimal Residual Method was used instead of CG in this case.

Contrary to the Euler method, any solution to (22) depends on the previous $\mathbf{I}_{m}$ (implicit in $\mathbf{g}^{\mathrm{n}}$ ). This makes the method particularly weak at initialization and fast changing exterior stimuli. A better solution can be obtained by precalculating $\mathbf{I}_{m}^{\mathrm{n}+\frac{1}{2}}$ in a predictor-corrector scheme. This will be referred as the Euler-Crank-Nicholson (ECN) method (see also Fig. 3(E,F)).

\subsection{Computer Setup}

The software solver was written in $\mathrm{C}++$ code, linked to the GNU-LGPL library "Cancer, Heart and Soft-Tissue Environment" (CHASTE). CHASTE is being actively developed by the Computational Biology Group at the Oxford Computing Laboratory [44]. This framework provides functionalities for the solution of PDEs and ODEs for the heart and other tissues but does not provide cell membrane representations. To include the concept of insulating membranes, various additions to the CHASTE package were added using standard object oriented programming techniques. These extended CHASTE's bidomain [48] and ion channel functionalities, implementing the model presented in section 2. In its current version, CHASTE Membrane has been in development for 21 months and can be obtained for free on http://www.cs.ox.ac.uk/chaste/download.html under the "Bolt-on Projects" section.

Two and three dimensional meshes were generated with the GNU-GPL tool Gmsh [49]. The neuron reconstruction presented in section 3 used NEURON's [50] Python's interface to parse the $1 \mathrm{D}$ geometry and Gmsh to generate the mesh. Custom written Python code was used for demarcation of the cell membranes and post-processing. Extra and intracellular spaces are effectively independent meshes that only share node positions at the interfaces. Example meshes are provided in the software toolbox. Paraview (Kitware Inc.) was used for visualization and sampled extraction of potentials over space. All simulations, except the example in Figure 8, were executed in a single processing core of an Intel@ Core 2 Quad $2.83 \mathrm{GHz}$ CPU, in a desktop computer with $4 \mathrm{~GB}$ RAM.

\section{Results}

\subsection{Convergence, stability and computa- tion time}

The solution scheme, introduced in the previous section, consist of two separate procedures: one solves (18) finding the electric potential in $\Omega$, and the current reaching the membrane $I_{m}$, under boundary conditions on $\partial \Omega$ and $\Gamma$; the other, adds up the membrane currents that result from the electric potential and ion fluxes, updating the membrane
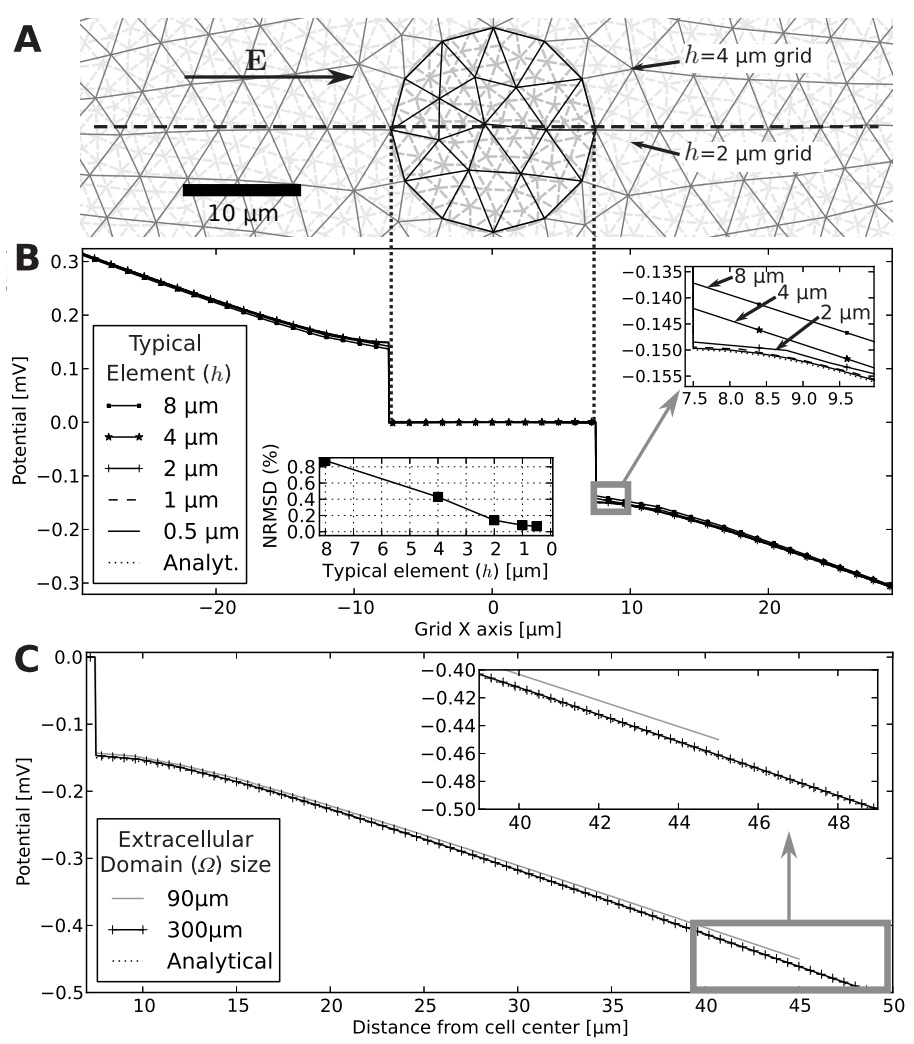

Figure 2: Convergence tests were performed for a $2 \mathrm{D}$ circular cell inside a $100 \mathrm{mV} / \mathrm{cm}$ extracellular potential gradient $\left(d=15 \mu \mathrm{m}, \sigma_{i}\right.$ $=5 \mathrm{mS} / \mathrm{cm}, \sigma_{e}=20 \mathrm{mS} / \mathrm{cm}, R_{m}=10^{3} \Omega \times \mathrm{cm}^{2}, C_{m}=1 \mu \mathrm{F} / \mathrm{cm}^{2}$, Dirichlet $\mathrm{BC} \Phi_{D}=-100 x$ on $\partial \Omega$ ) (A) Example of two of the spatial discretizations ( $h=4$ and $2 \mu \mathrm{m}$ ). (B) Comparison to the analytic solution at stationary state (dotted line) of the numerical solution for each $h$ along the mesh's $x$ axis (dashed horizontal line in $(\mathrm{A})$ ). The solution converges as displayed in the top right inset. The bottom middle inset presents the NRMSD to the analytical solution as the element size decreases. Neumann boundary conditions provide similar convergence rates (not displayed). The size of $\Omega$ was $300 \times 300 \mu \mathrm{m}$. (C) Boundary effects. Closer results to the infinite space analytical solution can be obtained with larger boundaries. The influence of a short squared $90 \times 90 \mu \mathrm{m}$ domain can be compared to a $300 \times 300 \mu \mathrm{m}$ one. A good choice of domain size has to be balanced with the number of mesh nodes. The cell membrane voltage can be noticeably affected with boundaries distances of 1 to 2 times the cell diameter (NRMSD $2.96 \%$ for $90 \mu \mathrm{m}$ and $0.69 \%$ for $300 \mu \mathrm{m})$. 
voltage $V_{m}$. The new $V_{m}$ represents an updated boundary condition on $\Gamma$. Given stationary boundary conditions, if $V_{m}$ over $\Gamma$ corresponds to a steady state value, the electric potential will correspond to the steady state distribution of potential in $\Omega$. In this stationary case the implementation of the spatial solver (Eq. (18)) can be tested, independent of time evolution (Eqs. (20), (21)).

Making use of this principle, it was assessed how the discretization of space influences the precision of the solution. For sufficiently fine spacing, the numerical solution should eventually converge towards the analytical. One of the cases where the analytical solution is known is a $2 \mathrm{D}$ circular cell stimulated by a homogeneous field turned on at time $t=0$ (see supplementary material). Figure 2(A,B) shows simulations for such a cell with diameter $d=15 \mu \mathrm{m}$. The numerical precision was judged by comparison of a numerical $\left(\phi_{n}\right)$ and an analytic solution $\left(\phi_{a}\right)$, quantified by the normalized root mean square deviation (Figure2(B), lower inset):

$$
N R S M D=\sqrt{\sum_{i=1}^{n}\left(\phi_{n, i}-\phi_{a, i}\right)^{2} / n} /\left(\phi_{a, \max }-\phi_{a, \min }\right) .
$$

As the typical grid spacing $h$ went from $8 \mu \mathrm{m}$ to $0.5 \mu \mathrm{m}$, the magnitude of the computed electric potential approached the analytic solution (Figs. 2(A,B)).

The analytic solution assumes an infinite extracellular space, so the analytical and numerical results disagreed in the proximity of the domain boundaries $\partial \Omega$. The influence of the boundary on the solution at the cell's surface is $2.96 \%$ for a distance to the boundary of $30 \mu \mathrm{m}$. At the distance of nine $d$ the error drops to negligible $0.69 \%$.

After confirming the accuracy of the first part of the numerical scheme, convergence was tested for the time development of the potential and membrane voltage as the time step was reduced. First, a configuration similar to that of figure 2 was used (Figure 3(A,B)). The extracellular field, imposed by Dirichlet boundary conditions, was turned on at $t=0$. For a mesh with a typical spacing of $h=1 \mu \mathrm{m}$, the numerical solution comes very close to the analytical after the transition phase. This is equivalent to the observation of Figure 2. However, the initial phase of the $V_{m}$ build-up is not captured properly (Figure $3(\mathrm{~A})$ ).

To achieve an accurate representation of the membrane voltage development it is necessary to reduce the time step. The numerical solution converges to the analytic solution as the time step is reduced first to $5 \mathrm{~ns}$ and then to $0.5 \mathrm{~ns}$ (Figs. $3(\mathrm{~A}, \mathrm{~B})$ ). Because a smaller time step also allows for finer grid spacing, the grid was improved moderately. This had no influence on the convergence (data not shown). It was found empirically, that the machine time per mesh node and per simulation time step (MTNT) was largely independent of the specific problem or the dimension. For the Euler forward solver used for Figure 3 (A) and (B), the MTNT amounts to 80 to $90 \mu \mathrm{s}$.

A similar MTNT (120 ps) was found for the next example, a $1 \mu \mathrm{m}$ thin and $80 \mu \mathrm{m}$ long cable under an axial field. The grid was generated with an intended grid spacing of $0.5 \mu \mathrm{m}$. A time step as small as 2 ns was necessary to achieve a stable solution. Due to the different morphology, the typical time of the $V_{m}$ build up is much larger than in the previous case. To compute a $300 \mathrm{~ms}$ time-course, the simulation took $26 \mathrm{~h}$ (explicit Euler method). With many hours of computation time for a single $80 \mu \mathrm{m}$ long cable, a timely treatment of realistic problems would not be possible with this approach. Using our insight into the different stability conditions for the Euler and Crank-Nicolson solvers (supplementary material) the later was implemented to benefit from the fact that the Crank-Nicolson solver tolerates orders of magnitude longer time steps keeping numerical stability (Figure

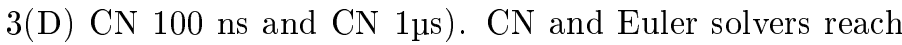
comparable precision when compared to a similar solution with the cable equation in perfectly conducting extracellular space [12] (Figure 3(D), dotted line). A combination of the computational steps that are used in the Euler and CrankNicolson approaches allows to compensate the short-comings (Sec. 2) of the Crank-Nicolson alone. The resulting EulerCrank-Nicolson solution is precise, even at times when the stimuli change, and allows for large time steps that would lead to numerical instability with the explicit Euler method alone (Figure 3(E), ECN $100 \mathrm{~ns}$ ). The instability of the explicit Euler method can be seen in the diverging zigzag lines in Figure 3(E,F) (Euler $100 \mathrm{~ns}$ ).

Stability of the explicit Euler scheme requires time steps in the order of $h C_{m} / \sigma$, where $C_{m} / \sigma$ is typically $\sim 10 \mathrm{~ns} / \mu \mathrm{m}$. This means that the a morphology with details in the 100 $\mathrm{nm}$ range demand $1 \mathrm{~ns}$ time steps (supplementary material). The implicit Crank-Nicolson scheme, on the other hand, is unconditionally stable for realistic parameters and can, theoretically, tolerate time-steps close to the membrane time constant. Convergence results for the different methods can be seen in the supplementary Table S.2.

\subsection{Representing neuron details}

With the application of the Crank-Nicolson solver to FEM problems with individual domains and insulating membranes, it seemed possible to treat realistic geometries and time scales within computation times of hours. This was put to a more rigorous test by the simulation of a $600 \mu \mathrm{m}$ long and only $0.5 \mu \mathrm{m}$ thick axon-like fiber under stimulation by a strong field $(1000 \mathrm{~V} / \mathrm{m})$. Cable endings are especially interesting for the effects of neuron stimulation, because, under spatially homogeneous conditions, endings are the places where the largest depolarization occurs $[31,51]$. The exact shape of the endings and the influence of this shape on the magnitude of the induced depolarization has not been studied.

To show the potential of our approach to represent fine details in the morphology and analyze their effect, three different meshes, representing differently shaped presynaptic boutons were created (Fig. 4(A)). To show the implications of a wide bouton for both phases: the very rapid, initial depolarization and the later equilibration of membrane currents, a simulation time of $8 \mathrm{~ms}$ was chosen. With a typical mesh spacing $h=0.25 \mu \mathrm{m}$ (imposed by the cable radius), the explicit Euler method required a time step of 4 ns to avoid divergence due to numerical instability. Even with adaptive meshes, that represent finer details only at the tip of the cable, the computation time with the Euler forward method was 8 weeks for the cable without an extended presynaptic bouton (Fig. 4(B)). When compared to the cable equation solution for the infinitely thin cable, the numerical solution displayed a small difference in the steady state membrane potential. This is likely an effect of the finite diameter. Overall, the NRSMD was $1 \%$. The same precision was obtained more than 230 times faster, when the Euler-Crank-Nicolson solver was used with a time step of $1 \mu \mathrm{s}$, demonstrating the importance of this approach (Fig. 


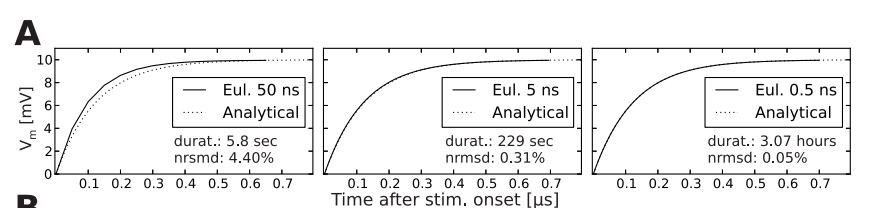

B

C

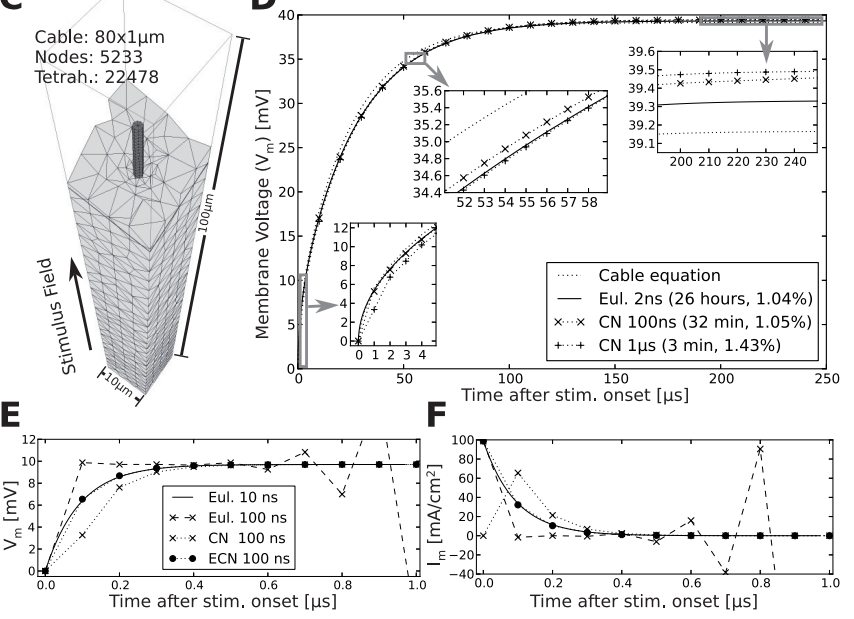

Figure 3: Convergence and stability of the time dependent solutions. (A) The layout is similar to Fig. $2\left(d=10 \mu \mathrm{m}, E=1 \times 10^{4} \mathrm{mV} / \mathrm{cm}\right.$, $\Omega: 400 \times 400 \mu \mathrm{m}$, initial $V_{m}(\theta, 0)=0$, same parameters $)$. The time development of $V_{m}$ is computed more and more precisely for smaller time steps (Euler method). The text shows simulation duration and $V_{m}$ error. (B) Section of the meshes used on A. Typical mesh spacing $h=\{1,0.5,0.25\} \mu \mathrm{m}$. (C) $3 \mathrm{D}$ mesh representing a short cable-like cell $\left(\sigma_{e}=\sigma_{i}=10 \mathrm{mS} / \mathrm{cm}, h=0.5 \mu \mathrm{m}\right.$, membrane properties as in 2). (D) Numerical solutions with different solvers and time steps. The computation time can be drastically reduced using the Crank-Nicolson (CN) approach. As a reference, the solution of the cable equation is shown. (E and F) A simulation similar to that in A. (E) The CN method alone has shortcomings when the exterior stimulus varies (e.g. step at $\mathrm{t}=0$ ). This is caused by the dependency of the method on previously estimated membrane currents $\left(I_{m}\right)$. Addition of one regular Euler step (ECN method) previous to the CN calculation, solves this problem. (F) The initial current $I_{m}$ is unknown and assumed zero on the $\mathrm{CN}$ method, consequently the first field calculations have a large error. The ECN method reaches a far better solution. Using the same time step, the Euler method alone does not lead to a stable solution.
$4(\mathrm{~B}))$.

To resolve the influence of the bouton shape on the depolarization in the initial phase, the time step of 1 us was too large. For this purpose use of adaptive time steps is more appropriate. In this way, the fast response at the beginning of the simulation and the slow response at steady state can be captured. With this approach computation times can be reduced further (Fig. $4(\mathrm{C}, \mathrm{D})$ ). For this example, the points at which the time steps were switched had been chosen manually, guided by the magnitude of the membrane currents $I_{m}$. This approach is motivated by the fact that the change in the potential depends on $I_{m}$ in Eq. (21). For large membrane currents, the time steps have to be chosen short in order to capture the rapid changes in the potential. This scheme can be extended into an automatically adapting time step: the values of $I_{m}$ are available during the computation, as equation (19) is solved. Depending on the recent $I_{m}$ values, the next time step can be chosen.

The different bouton shapes influence the membrane potential $V_{m}$ and the extracellular potential. The presence of a synaptic bouton increases the membrane depolarization during the initial phase, reflecting the rapid redistribution of charge in the bouton compartment. In the steady state, after several milliseconds, the larger surface of the bouton leads to a larger ionic current. This diminishes the depolarization, but increases the extracellular potentials (Fig. $4(\mathrm{E})$ ). The reduced polarization of larger boutons could be used to selectively activate those boutons in a preparation that has smaller synaptic endings.

\subsection{Deficiencies of the cylinder segmenta- tion}

The vast majority of studies of neuronal dynamics is based on calculations that approximate the cell morphology by cylindrical sections. The membrane potential in each section develops then according to the cable equation. This approximation is very powerful and its application dominates the picture of electrical processes in excitable cells that we have today. Even the values for a basic parameter such as the cytosolic conductivity $\left(\sigma_{i}\right)$ are obtained by matching measured voltage differences with voltage differences predicted from cable models [52]. The approximation of neurons as concatenations of cylindrical cables breaks down on the micrometer scale, for instance when fine structures such as synaptic boutons or dendritic spines deviate from axial symmetry. While attempts can be made to account for the presence of extra membrane in the spines (by adjusting the surface capacitance and conductance obtaining valid expressions for the average voltage across a longer segment), on the microscopic scale only a right representation of the morphological details can give correct simulations of the membrane voltages in these small compartments.

Even larger problems arise, when the relevant physical processes do not evolve within the axial/radial coordinates of the cable segments. To describe the interaction between a cylindrical segment and the extracellular field, only the field's component perpendicular to the cylinders faces is used to compute the cable equation [32]. This works well, as long as the approximation of a very thin cable is appropriate, however it completely fails for spherical structures like cell bodies (Figure 5). The key problem is the fact that only the area of the cylinder's faces determines the magnitude of 

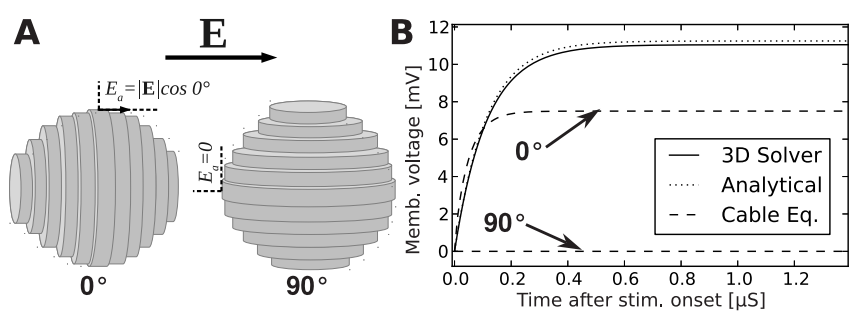

Figure 5: Deficiencies of the cylinder segmentation and the cable equation $(\mathrm{CEq})$ for extended structures such as the soma. (A) A spherical body (SB) can only be represented as a collection of cylinders in the CEq. Under a homogeneous electric field $\mathbf{E}$, stimulation of each cylinder corresponds to simultaneous and opposite current injections in the two cylinder ends. This injection is proportional to the projection of the field along the cylinder's axis. As the SB is rotated $\left(0^{\circ}\right.$ to $\left.90^{\circ}\right)$, the projection of the electric field vanishes despite the symmetry of the underlying problem. (B) Even at $0^{\circ}$, the CEq is inaccurate representing stimulation of a SB. Upper traces correspond to the analytical [53] and numerical membrane voltage response of the $3 \mathrm{D}$ SB calculated with the tool $\left(d=15 \mu \mathrm{m}, R_{m}=10^{3} \Omega \times \mathrm{cm}^{2}, C_{m}=1 \mu \mathrm{F} / \mathrm{cm}^{2}, \sigma_{e}=\sigma_{i}=10 \mathrm{mS} / \mathrm{cm}\right.$, $|\mathbf{E}|=10^{4} \mathrm{mV} / \mathrm{cm}, V_{m}$ measured at the right most point). The CEq does not represent the cylinder faces so their extra resistance and capacitance are not accounted for.
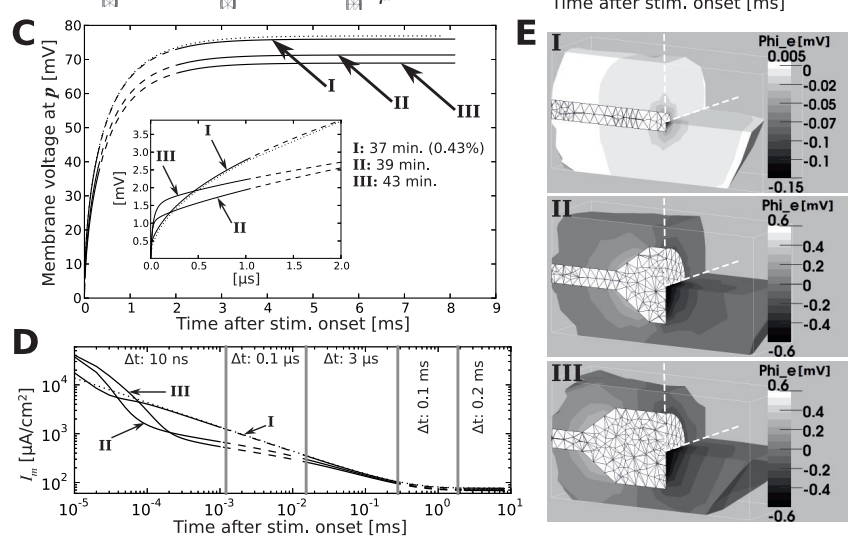

Figure 4: Stimulation of cables with differently shaped bouton endings. (A) Meshes representing three variations (I, II, III) on $600 \mu \mathrm{m}$ cables $\left(\sigma_{e}=10 \mathrm{mS} / \mathrm{cm}, \Omega: 720 \mu \mathrm{m} \times 30 \mu \mathrm{m}\right.$ diameter cylinder, not displayed, $\sigma_{i}=5 \mathrm{mS} / \mathrm{cm}, R_{m}=10^{3} \Omega \times \mathrm{cm}^{2}, h=0.25 \mu \mathrm{m}$ ). (B) Response at point $p$ for geometry I to a $1000 \mathrm{~V} / \mathrm{m}$ field. At $4 \mathrm{~ns}$ time steps the Euler method required 58 days. When larger times steps were used, the solution diverged (not shown). With ECN a 11p time-step could be used and computation time dropped to hours. The related analytical solution of the cable equation (CEq) is displayed for comparison. The $1 \mathrm{mV}$ difference in steady state reflects the finite area of the cable caps, that is ignored in the CEq. Larger cap areas tend to lower the voltage (as seen in C). NRMSD are comparable for Euler and ECN. (C) Response at $p$ for geometries I, II and III, computed with an adaptive scheme. The switching of time-steps further reduced simulation time while resolving the waveform of the initial response (inset). Changes in the time-step are denoted by changes in the line styles. Simulation times are given and the NRMSD to the analytical solution is given in parenthesis. (D) Log-Log plot of the membrane currents $I_{m}(p)$ from C. The five part adaptive schedule $(\Delta t)$ was chosen qualitatively from variations in current slope. (E) Cross-section of the extracellular potential at steady state $(t>6 \mathrm{~ms})$ after subtraction of the stimulus potential. After deactivation of the capacitive current, the ionic current distorts the extracellular potential especially when there is a bouton. the stimulus, while the area of the cylinders side determines the capacitance and conductance of the membrane. Face area and side area cannot at the same time match the effective areas of the sphere. This causes a erroneous result for the effect of extracellular stimulation of a cell body, even in the case that the field is oriented along the cylinders' axes (approximate $30 \%$ error in $5(\mathrm{~B}), 0^{\circ}$ ). When the orientation is changed, the approximation breaks down completely (Figure $5(\mathrm{~A}, \mathrm{~B}))$. This is a fundamental problem of the approximation by cylindrical segments and can only be solved when the membrane surface itself determines the orientation of the electric fluxes and not the axis of a segment.

\subsection{Modeling multiple cells}

In the brain, neurons are not surrounded by empty, infinitely conductive space. Neurons and supporting cells are densely packed so that only about $20 \%$ of the brain volume represent extracellular space [55]. Determining the influence of other cell bodies in the potential signal of the neuron is key to understand the shape of extracellularly recorded action potentials and in general, of local potentials. Collections of cells surrounding a neuron represent resistance and capacitance that has to be accounted for. The effect of this distribution in the extracellular space is still debated [56].

The tool presented in this work can be used to model collections of passive and active cells at close distances, resolving the extracellular potential during their activity. To illustrate the importance of representing other cell bodies, a model of a tight packing of $2 \mathrm{D}$ cells under stimulation was implemented (Fig. 6).

Under a homogeneous electric field, a collection of cells in this configuration behaves essentially as a series of capacitors and resistors fed by a constant voltage source. When the voltage is turned on, current flows freely from one end of the circuit to the other and the potential decays in the resistors (extra and intra-cellular spaces). As charge builds up in the capacitors (the cell membranes), the current flow decreases and the membrane voltage increases. At steady state, the potential difference across the entire equivalent "circuit" is determined by the first and last membranes along 

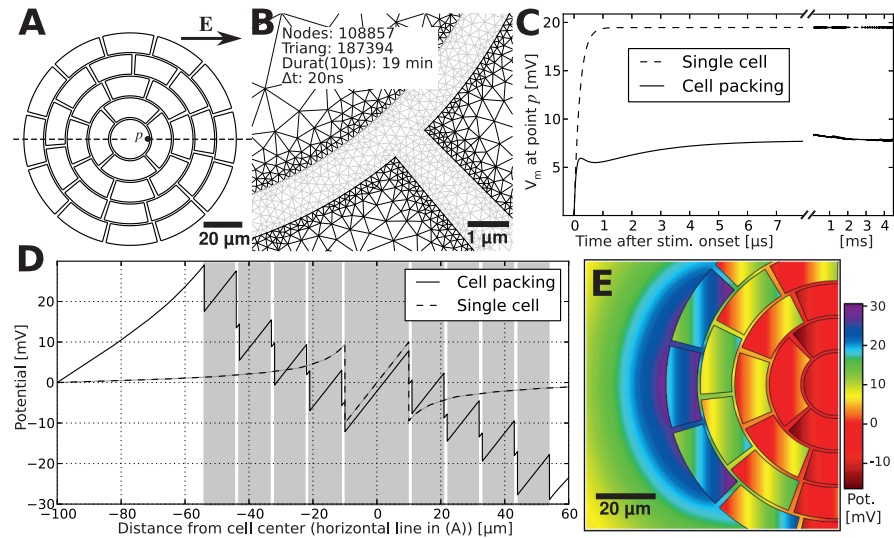

Figure 6: Tight cell packing strongly influences the effect of extracellular stimulation. (A) The two-dimensional cell arrangement studied. In the single-cell case, only the central cell $(d=20 \mu \mathrm{m})$ existed. In the cell-packing case, it was surrounded by 4 rings of closely packed cells. Parameters used $\sigma_{e}=\sigma_{i}=10 \mathrm{mS} / \mathrm{cm}, R_{m}=10^{3} \Omega \times \mathrm{cm}^{2}, C_{m}=1 \mu \mathrm{F} / \mathrm{cm}^{2}$ all. (B) Detail of the mesh at inter-cell gaps. The inter-cellular space (gray mesh) was finely detailed to improve accuracy of the solution $(h=150 \mathrm{~nm})$. Simulation duration for $10 \mu \mathrm{s}$ was $19.6 \mathrm{~min}$. (C) Under stimulation with a homogeneous electric field $(1000 \mathrm{~V} / \mathrm{m})$ the membrane voltage develops very different for the the single- and the cellpacking case. The voltage response at the central cell is greatly reduced by the shielding. In addition, the time course of the response exhibits a combination of the primary cell time constant (200ns, [54]) and the membrane time constant (1ms). A maximum is reached around $200 \mu \mathrm{s}$ $(8.4 \mathrm{mV})$ but the final steady state is reached only after $\mathrm{t} \approx 4 \mathrm{~ms}$ (broken abscissa, simulated with larger time-steps). (D) The potential $\Phi-\Phi_{E}$, at $t=1 \mathrm{~ms}$, along the horizontal axis of panel A. Subtraction of the primary field $\Phi_{E}$, i.e. the $1000 \mathrm{~V} / \mathrm{m}$ gradient, reveals the large secondary field outside the cell cluster and between cells. Shaded areas represent the intracellular space. (E) Potential color map at $t=1 \mathrm{~ms}$. Effects from the flow of currents at the gaps can be observed together with the distortions of up to $30 \mathrm{mV}$ at the exterior of the ring.

the field direction. If the various capacitors and resistors have similar properties, the potential drops are distributed more or less equally across them $(6(\mathrm{D}))$. The main effect is that the membrane voltage of the cell targeted for stimulation is lower, compared to that of a isolated cell (Fig. 6(C)). For neuro-stimulation techniques such as the transcranial magnetic stimulation, that aims at supra-threshold activation of the cell and utilizes rapidly rising, strong stimuli as in this example, the shadowing effect and its consequences (e.g. secondary fields caused by the inhomogeneities of tissue [29]) can determine success or failure of stimulation. The formation of local secondary fields as visible in the potential gradients along the gaps between cells is not represented by the "far-field" approach of the line-source method.

The case presented in Fig. 6 is equivalent to the transversal stimulation of a bundle of "infinitely" long cells in $3 \mathrm{D}$. In cable-equation solvers, such as NEURON, transversal stimulation exerts no effect on the membrane voltage, just as shown in Figure 5. As the finite element method does not impose any symmetry assumptions, it can represent the distortions of the extracellular potential caused by even more local obstructions, like a single small spherical cell body next to an active cell. The example in Fig. 7 demonstrates the influence of neighboring cells on the extracellular potentials during an action potential. The slightly larger sphere in the center represents a neuronal soma, that would receive a depolarizing current during the initial phase of an action potential. In reality that would be the lateral current originating at the axon initial segment. This typically depolarizes the soma at a rate of up to $400 \mathrm{~V} / \mathrm{s}$. In the simulation, the
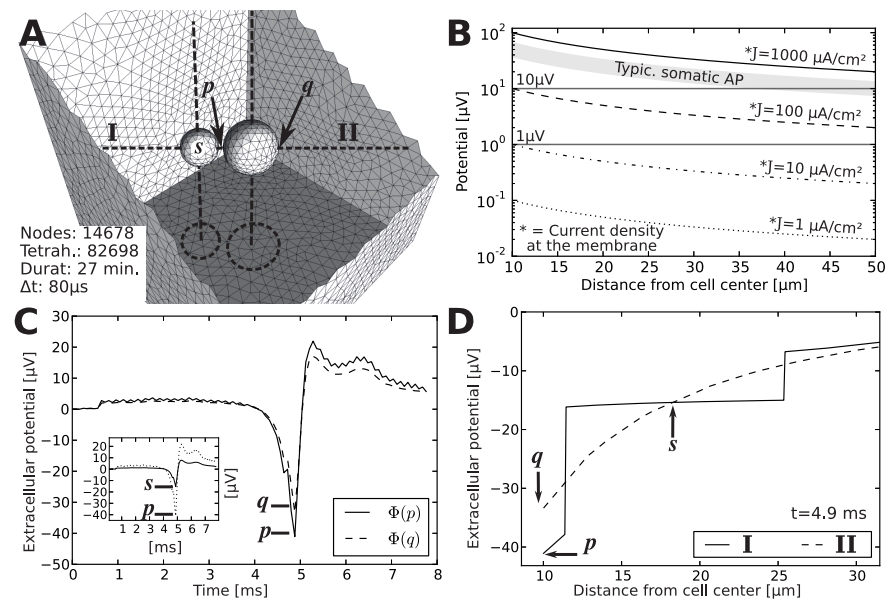

Figure 7: The extracellular action potential and effects of a nearby cell. (A) 3D geometry of the virtual experiment. A spherical cell $(d=20 \mu \mathrm{m})$ was located in the center of a $100 \times 100 \times 100 \mu \mathrm{m}$ cubic domain, $\sigma_{e}=10 \mathrm{mS} / \mathrm{cm}$. A second smaller cell $(d=14 \mu \mathrm{m})$ was placed $1.5 \mu \mathrm{m}$ next to it. The smaller cell had passive properties $\left(R_{m}=10^{3} \Omega \times \mathrm{cm}^{2}, C_{m}=1\right.$ $\mu \mathrm{F} / \mathrm{cm}^{2}, \sigma_{i}=10 \mathrm{mS} / \mathrm{cm}$, resting potential zero), while the larger cell was set to emit an extracellular action potential with typical amplitudes and a waveform taken from the results of Fig. 8. (B) The spatial falloff of extracellular potential for a $d=20 \mu \mathrm{m}$ cell as a function of the membrane current density $\mathrm{J}$ and distance. The potential formula used was: $\Phi_{e}(r)=d^{2} \mathrm{~J} / 4 \sigma_{e} r$. Typical current densities on the soma at the peak of sodium influx are around -200 to $-800 \mu \mathrm{A} / \mathrm{cm}^{2}$ (theoretical estimate [26, 27] and recordings performed by AN). With $\sigma_{e}=10 \mathrm{mS} / \mathrm{cm}$ this accounts for a potential near the membrane of $\sim 50 \mu \mathrm{V}$. (C) Extracellular potential at point $p$ and $q$ during $8 \mathrm{~ms}$. The inset compares the potential at point $p$ with the intracellular potential at the center of the small cell $(s)$. (D) Potential along axes I, II at the peak of the extracellular potential $(\mathrm{t}=4.9 \mathrm{~ms})$. Axis I pierces the small cell. The portion of the small cell's membrane closer to the large cell has its own voltage and distorts the extracellular space potential.

current is supplied by an injection inside the cell with a current that varies over time, mimicking a real action potential current shape. Note this also illustrates the possibility to stimulate by current injection at arbitrary locations, which is a feature that would give the user the possibility to more precisely model stimulation via a pipette. When the waveform of the resulting extracellular potentials between the active and the passive cell is compared to the extracellular potential at the opposite site, the amplifying influence of the adjacent membrane can be seen (Fig. $7(\mathrm{~A}, \mathrm{C})$ ). At the peak amplitude, the potential is amplified by $8 \mu \mathrm{V}$. (Fig. $7(\mathrm{D})$ ). This difference is determined by the nearby cell, whose own response and membrane voltage (Fig. $7(\mathrm{C})$, inset) distorts the potential in this region. Amplitudes of tens of microvolts can be typical of extracellular action potential recordings at the given conductivity (Fig. $7(\mathrm{~B})$ ). With realistic complementary shapes of neighboring cells, the magnitude of the amplification would be much larger. The $8 \mu \mathrm{V}$ effect on the potential is well resolved by the method although its magnitude is much smaller than the typical membrane voltage (tens of $\mathrm{mV}$ ).

\subsection{Realistic setups}

Comprehending effects of EPs and in general of the electrical functions of the cell requires realistic geometries and a realistic distribution of voltage dependent channels. Construction of new stimulation and recording devices [22, 23, 24, 25] such as those required to advance nerve- and brain-computer interfaces demand modeling environments that can model this 
heterogeneity. Simulation methods able to model biological tissue and artificial materials more realistically will be fundamental to lower development and experimentation costs.

To demonstrate the capabilities of the method to represent realistic setups, in Fig. 8, simulation of a cultured neuron equipped with voltage gated ion channels, on top of a glass coverslip is presented. This preparation is interesting for the development of multi-unit micro electrode arrays [57, 17]. In [17], a similar setup was presented, consisting of a 3D ball and a stick neuron with distributed voltage dependent channels. A commercial software tool was used in this work and the details of the numerical implementation were not accessible.

The morphology presented in Figure 8, was generated from parts of the reconstruction of cell D151 from [27] (see methods). The bottom of the cell was flattened representing the interface with the glass, at a distance of $0.8 \mu \mathrm{m}$. The complete mesh consisted of 21732 nodes and 86716 tetrahedra in a domain of dimensions $340 \times 200 \times 60 \mu \mathrm{m}$. An adaptive mesh was used, with refinements near and inside the cell of down to $300 \mathrm{~nm}$. A heterogeneous distribution of sodium and potassium voltage dependent channels was set along the cell. The sodium conductances for the dendrite, soma/hillock, initial segment, and the rest of the axon were respectively $10,20,120,20 \mathrm{mS} / \mathrm{cm}^{2}$. The potassium conductances, for the same sections, were 10, 20, 30 and 20 $\mathrm{mS} / \mathrm{cm}^{2}$. Mammal kinetics at $23^{\circ}$ were used [58].

The simulation of $8 \mathrm{~ms}$ of activity in this setup with the reference software required only $45 \mathrm{~min}$ on a single processor core (see methods). The solver was configured to use the ECN method with a fixed time-step of 80 ss. A larger, 100 ms simulation was executed with the same parameters (Fig. $8(\mathrm{~F})$ ), requiring $6 \mathrm{~h} 27 \mathrm{~min}$. Computation time could be reduced to $4 \mathrm{~h} 16 \mathrm{~min}$ when the computation was distributed to two processors in parallel, and to $3 \mathrm{~h} 21 \mathrm{~min}$ in four processors. However the mesh was not optimally partitioned (see discussion). A video of this simulation is provided on-line as supplementary material.

\section{Discussion}

Our quantitative understanding of electrophysiology is based on the concepts of the Hodgkin-Huxley model and the cable equation. This concepts describe the behavior of isolated cells inside an isopotential space. Extracellular potentials can be added to this picture following the concept of the activating function [12], however there are fundamental limitations to the validity of this approach for structures that are not thin, such as somata (5). The intrinsic feedback between endogenous extracellular fields and cellular activity is never captured by the traditional approaches, as always only one direction, e.g. membrane currents to potentials, is captured. When the physical problem is formulated in a way that takes the interaction between currents and fields into account, this results in partial differential equations with non-linear boundary conditions. While a few simulations have been published that addressed this problem, they focused on either stimulation of cells by extracellular fields (passive membrane: [59], two dimensions: [15]) or on the detection of endogenous fields by planar electrodes [17]. Moreover, proprietary software was used. To our knowledge no finite element software is openly available, which would pro- vide a self-consistent picture of the mutual feedback between currents and potentials.

The presented numerical method allows detailed simulations of the electric activity of excitable cells and their interaction with extracellular potentials. Membranes are explicitly modeled. They can have arbitrary shapes on realistic sub-micrometer scales and contain voltage dependent conductances. The extracellular space is an integral part of the mathematical structure. Therefore the interplay between membrane potential, membrane currents and extracellular fields is intrinsic to the simulations, and not imposed by additional boundary conditions. The method is able to solve problems with mixed boundary conditions, represent geometric details, and allows relatively long simulated times in much shorter computation times than previously possible. The code of a reference implementation, CHASTE Membrane, is released as open source, and builds upon the open source initiative CHASTE [44]. Along with CHASTE, other modern, mainstream high performance and parallel, open computing libraries are used, including PETSc (Argonne National Lab.), MPI (Message Passing Interface standard), HDF5 (NCSA and others), VTK (Kitware Inc.) and CellML (Europe's Virtual Physiological Human Project). The use of open and well supported libraries ensures their continued development and optimization. CellML grants access to a public library of ion channel kinetics definitions and eases the implementation of new ion channel models (http://www.cellml.org/).

Numerical precision and convergence of the method were demonstrated using analytically tractable problems. The basic stability criterion turned out to be linearly related to the typical grid spacing $h$, unlike the $h^{2}$ dependence found for the heat equation, propagation of action potentials $[60,34]$ and bidomain models of electric activity in the heart [43]. The linear dependence on $h$ is a consequence of the separation of the time and space equations and the dimensionality of the problem: only the component of potential gradients that is oriented perpendicular to the membrane contributes to the membrane current $I_{m}$. This directionality is also the reason why the linear relation between the typical grid spacing $h$ and the largest allowed time step also holds for three dimensions. Replacing the Euler forward solver of CHASTE by the implicit and numerically more stable Crank-Nicholson solver, it was possible to increase the simulation time step and thereby reduce computation time by orders of magnitude. For the Crank-Nicolson solver, the time step can approach the membrane time constant, while for the Euler forward solver the much shorter cell time constant was limiting [54]. This finding is fundamental for long time simulations where the main observed phenomena lie mainly in the membrane time constant regime. To reduce computation time further, adaptive time steps and adaptive meshing were used, to reduce the number of time steps and nodes, without compromising precision at structural details and rapid temporal changes.

In the software implementation, standard finite element techniques were employed to solve the numerical problem. Interaction with the cells is possible via boundary conditions, that allow for instance to fix the voltage at a membrane element or the current, that flows into the membrane. This is comparable to the voltage clamp and current clamp point processes that are available for user interaction in cable equation simulation tools such as NEURON or GENESIS. 

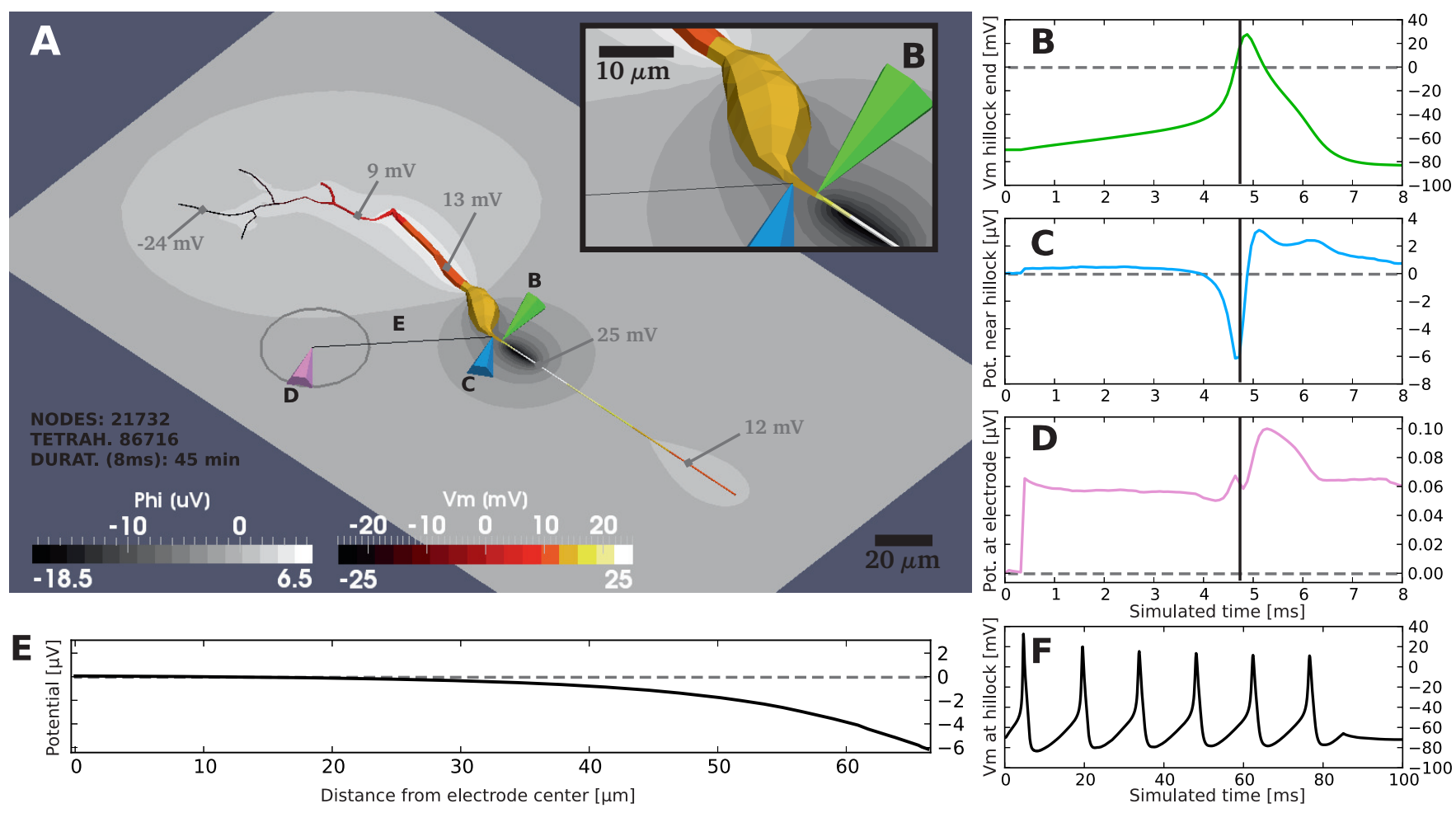

Figure 8: Potentials in and around a cultured neuron firing action potentials (AP). (A) The morphology of the simulated neuron is derived from an actual reconstruction (see text). Color/grayscale indicate the membrane potential $V_{m}$ and extracellular potential $\Phi_{e}$ at the bottom of the dish in a snapshot just after initiation of the AP $(t=4.72 \mathrm{~ms}$, vertical line in B-D, $\Delta t=40 \mu \mathrm{s})$. The cell was stimulated by injection of $100 \mathrm{pA}$ into the soma beginning at $\mathrm{t}=0.3 \mathrm{~ms}$. The AP started in the axon initial segment (approx. $20 \mu \mathrm{m}$ away from the soma) which has a higher concentration of sodium and potassium channels (see text). Due to the low axon diameter $(0.67 \mu \mathrm{m})$ the absolute current at the axon initial segment is not larger than the somatic current. However, the large lateral current flowing to the soma causes a large extracellular potential (grayscale, see detail in inset). (B) Time trace of an intracellular recording with a "virtual pipette" located at the end of the axon hillock (marked as B in the main figure). Note that inserting a pipette in this point would be extremely challenging in a real preparation. (C) Time trace of the extracellular potential just outside the axon hillock. The negative peak of the potential corresponds to the peak of extracellular current flowing on to the membrane during sodium influx. (D) Extracellular potential as it would have been picked up by a surface electrode to the left of the soma (gray circle, marked as D). The larger components of this signal are the stimulus pulse (starting at $\mathrm{t}=0.3 \mathrm{~ms}$ ) and potassium activation at the soma and dendrite $(\mathrm{t}=5.2 \mathrm{~ms})$. (E) Extracellular potential along the line between markers $\mathrm{D}$ and $\mathrm{C}(\mathrm{t}=4.72 \mathrm{~ms})$. (F) $100 \mathrm{~ms}$ of activity during current injection ( $84 \mathrm{~ms}$, same parameters). This computation took 6 hours and 27 minutes with a time-step $\Delta t=100 \mu \mathrm{s}$ 
Going beyond the possibilities of those tools (NEU$R O N / G E N E S I S$ ), the finite element approach allows the implementation of other physical elements important for neural recording and stimulation, including extraneous elements such as glass pipettes or recording electrodes. Heterogeneous distribution or properties of ion channels can be implemented on a triangle by triangle basis, without imposing a radial symmetry. These are important features, necessary to understand for instance the heterogeneity of the shapes of extracellularly recorded action potentials $[57,61]$. But the most powerful feature that this software offers over the capabilities of cable equation solvers, is the explicit definition of extracellular space. This allows a direct examination of effects like ephaptic coupling not only on large scales but also for directly opposing membranes, conductivity heterogeneities and anisotropies in the extracellular space, when the assumptions of the line source approach break down.

Despite the large improvements with respect to time step and computation time, finite element simulations do not have the potential to fully replace tools such as $N E U R O N$ or GENESIS for problems, that are insensitive to the limited conductance of extracellular space, the detailed geometry of dendrites or the heterogeneity of ion channel densities. But, with mounting evidence for the synchronization of action potentials by extracellular potentials $[3,2,1]$ and the progress in neuro-stimulation techniques, many questions arise that cannot be comprehensively addressed under the assumptions of the cable equation. In this area, the presented method can enhance the understanding of neural activity, stimulation of neurons, and recording from neurons.

The examples presented here were intended to demonstrate possible applications and give an idea of the computation times involved. They focused on the effects of crammed extracellular space and the interaction between extracellular fields and neuronal activity on realistic spatial scales and with realistic parameters. Numerous other problems can be addressed by simulations with this method. Starting with small geometries as a study of depolarization of spines of different morphology, all the way to very large systems like the cross-talk of local field potentials and electrical activity of hundreds of tightly packed cells.

Several steps have already been taken to shorten the computation time further. We proposed to use the magnitude of the membrane currents as the criterion to automatically chose appropriate time steps. The structures of the software code already allows this run-time alteration of the time-step. Simulation of intrinsic neuronal activity will greatly benefit from this adaptation scheme and the use of the CrankNicolson solver because currents and electric fields change on timescales much larger than the cell time constant, which is important mostly for the rapid fields imposed by neurostimulation methods.

This method was built from the beginning with parallelism in mind and the software framework supports distributed solution of linear systems and distributed meshes. Initial tests have been performed and the most important aspect now is the development of better partition algorithms for the main mesh. It has to be automatically determined whether it is preferred to partition a given cell membrane or rather to keep as much of possible of the cell in a given parallel machine. The greatest benefits from parallelization will obviously come from distribution of large meshes as the time evolution of the problem is tightly coupled.

\section{References}

[1] Costas A. Anastassiou, Rodrigo Perin, Henry Markram, and Christof Koch. Ephaptic coupling of cortical neurons. Nature neuroscience, 14(2):217-223, February 2011.

[2] Flavio Fröhlich and David A. McCormick. Endogenous electric fields may guide neocortical network activity. Neuron, 67(1):129-143, July 2010.

[3] Simal Ozen, Anton Sirota, Mariano A. Belluscio, Costas A. Anastassiou, Eran Stark, Christof Koch, and György Buzsáki. Transcranial electric stimulation entrains cortical neuronal populations in rats. The Journal of neuroscience : the official journal of the Society for Neuroscience, 30(34):11476-11485, August 2010.

[4] Sarah H. Lisanby, Thomas E. Schlaepfer, Hans-Ulrich Fisch, and Harold A. Sackeim. Magnetic Seizure Therapy of Major Depression. Arch Gen Psychiatry, 58(3):303-305, March 2001.

[5] Mark Hallett. Transcranial Magnetic Stimulation: A Primer. Neuron, 55(2):187-199, July 2007.

[6] R. E. Gross and A. M. Lozano. Advances in neurostimulation for movement disorders. Neurological research, 22(3):247-258, April 2000.

[7] M. A. Nitsche and W. Paulus. Excitability changes induced in the human motor cortex by weak transcranial direct current stimulation. The Journal of physiology, 527 Pt 3(3):633-639, September 2000.

[8] Ryota Kanai, Leila Chaieb, Andrea Antal, Vincent Walsh, and Walter Paulus. Frequency-dependent electrical stimulation of the visual cortex. Current biology : $C B, 18(23): 1839-1843$, December 2008.

[9] Tamás Z. Kincses, Andrea Antal, Michael A. Nitsche, Orsolya Bártfai, and Walter Paulus. Facilitation of probabilistic classification learning by transcranial direct current stimulation of the prefrontal cortex in the human. Neuropsychologia, 42(1):113-117, January 2004 .

[10] Felipe Fregni, Paulo S. Boggio, Michael Nitsche, Felix Bermpohl, Andrea Antal, Eva Feredoes, Marco A. Marcolin, Sergio P. Rigonatti, Maria T. A. Silva, Walter Paulus, and Alvaro Pascual-Leone. Anodal transcranial direct current stimulation of prefrontal cortex enhances working memory. Experimental Brain Research, 166(1):23-30, September 2005.

[11] Agnes Flöel, Nina Rösser, Olesya Michka, Stefan Knecht, and Caterina Breitenstein. Noninvasive Brain Stimulation Improves Language Learning. Journal of Cognitive Neuroscience, 20(8):1415-1422, February 2008.

[12] Frank Rattay. Analysis of models for external stimulation of axons. IEEE Transactions on Biomedical Engineering, 33(10):974-977, 1986.

[13] E. C. Fear and M. A. Stuchly. Modeling assemblies of biological cells exposed to electric fields. IEEE transactions on bio-medical engineering, 45(10):1259-1271, October 1998. 
[14] Gary R. Holt and Christof Koch. Electrical Interactions via the Extracellular Potential Near Cell Bodies. Journal of Computational Neuroscience, 6(2):169-184, March 1999.

[15] Wenjun Ying and Craig S. Henriquez. Hybrid Finite Element Method for Describing the Electrical Response of Biological Cells to Applied Fields. IEEE Transactions on Biomedical Engineering, 54(4):611-620, April 2007.

[16] Christine Voßen, Jens Eberhard, and Gabriel Wittum. Modeling and simulation for three-dimensional signal propagation in passive dendrites. Computing and $\mathrm{Vi}$ sualization in Science, 10(2):107-121, June 2007.

[17] Céline Moulin, Alain Glière, Daniel Barbier, Sebastien Joucla, Blaise Yvert, Pascal Mailley, and Régis Guillemaud. A new 3-D finite-element model based on thinfilm approximation for microelectrode array recording of extracellular action potential. IEEE transactions on bio-medical engineering, 55(2 Pt 1):683-692, February 2008.

[18] Scott F. Lempka, Matthew D. Johnson, Michael A. Moffitt, Kevin J. Otto, Daryl R. Kipke, and Cameron C. McIntyre. Theoretical analysis of intracortical microelectrode recordings. Journal of neural engineering, 8(4):045006+, August 2011.

[19] Tianhe C. Zhang and Warren M. Grill. Modeling deep brain stimulation: point source approximation versus realistic representation of the electrode. Journal of Neural Engineering, 7(6):066009+, December 2010.

[20] E. J. Peterson, O. Izad, and D. J. Tyler. Predicting myelinated axon activation using spatial characteristics of the extracellular field. Journal of neural engineering, 8(4):046030+, August 2011.

[21] H. G. Meijer, M. Krupa, H. Cagnan, M. A. Lourens, T. Heida, H. C. Martens, L. J. Bour, and S. A. van Gils. From Parkinsonian thalamic activity to restoring thalamic relay using deep brain stimulation: new insights from computational modeling. Journal of neural engineering, 8(6), December 2011.

[22] M. Djilas, C. Olès, H. Lorach, A. Bendali, J. Dégardin, E. Dubus, G. Lissorgues-Bazin, L. Rousseau, R. Benosman, S. H. Ieng, S. Joucla, B. Yvert, P. Bergonzo, J. Sahel, and S. Picaud. Three-dimensional electrode arrays for retinal prostheses: modeling, geometry optimization and experimental validation. Journal of Neural Engineering, 8(4):046020+, June 2011.

[23] Ling Wang, Michael Riss, Jennifer Olmos O. Buitrago, and Enric Claverol-Tinturé. Biophysics of microchannel-enabled neuron-electrode interfaces. Journal of neural engineering, 9(2), April 2012.

[24] Hsin-Yi Y. Lai, Lun-De D. Liao, Chin-Teng T. Lin, Jui-Hsiang H. Hsu, Xin He, You-Yin Y. Chen, JyhYeong Y. Chang, Hui-Fen F. Chen, Siny Tsang, and Yen-Yu Y. Shih. Design, simulation and experimental validation of a novel flexible neural probe for deep brain stimulation and multichannel recording. Journal of neural engineering, 9(3), June 2012.
[25] James J. FitzGerald, Natalia Lago, Samia Benmerah, Jordi Serra, Christopher P. Watling, Ruth E. Cameron, Edward Tarte, Stéphanie P. Lacour, Stephen B. McMahon, and James W. Fawcett. A regenerative microchannel neural interface for recording from and stimulating peripheral axons in vivo. Journal of Neural Engineering, 9(1):016010+, January 2012.

[26] Claude Bédard, Helmut Kröger, and Alain Destexhe. Modeling Extracellular Field Potentials and the Frequency-Filtering Properties of Extracellular Space. Biophysical Journal, 86(3):1829-1842, March 2004.

[27] Carl Gold, Darrell A. Henze, Christof Koch, and György Buzsáki. On the Origin of the Extracellular Action Potential Waveform: A Modeling Study. Journal of Neurophysiology, 95(5):3113-3128, May 2006.

[28] Klas H. Pettersen and Gaute T. Einevoll. Amplitude variability and extracellular low-pass filtering of neuronal spikes. Biophysical journal, 94(3):784-802, February 2008 .

[29] R. Plonsey. The nature of sources of bioelectric and biomagnetic fields. Biophysical journal, 39(3):309-312, September 1982 .

[30] Stefan Luther, Flavio H. Fenton, Bruce G. Kornreich, Amgad Squires, Philip Bittihn, Daniel Hornung, Markus Zabel, James Flanders, Andrea Gladuli, Luis Campoy, Elizabeth M. Cherry, Gisa Luther, Gerd Hasenfuss, Valentin I. Krinsky, Alain Pumir, Robert F. Gilmour, and Eberhard Bodenschatz. Low-energy control of electrical turbulence in the heart. Nature, 475(7355):235-239, July 2011.

[31] B. J. Roth and P. J. Basser. A model of the stimulation of a nerve fiber by electromagnetic induction. IEEE transactions on bio-medical engineering, 37(6):588-597, June 1990.

[32] S. S. Nagarajan, D. M. Durand, and E. N. Warman. Effects of induced electric fields on finite neuronal structures: a simulation study. IEEE transactions on bio-medical engineering, 40(11):1175-1188, November 1993.

[33] Cameron C. McIntyre, Warren M. Grill, David L. Sherman, and Nitish V. Thakor. Cellular Effects of Deep Brain Stimulation: Model-Based Analysis of Activation and Inhibition. J Neurophysiol, 91(4):1457-1469, April 2004 .

[34] Robert Plonsey and Roger C. Barr. Bioelectricity: A Quantitative Approach. Springer, 3rd edition, June 2007.

[35] Sébastien Joucla and Blaise Yvert. Improved Focalization of Electrical Microstimulation Using Microelectrode Arrays: A Modeling Study. PLoS ONE, 4(3):e4828+, March 2009.

[36] K. W. Altman and R. Plonsey. Analysis of excitable cell activation: relative effects of external electrical stimuli. Medical \& Biological Engineering \& Computing, 28(6):574-580, November 1990. 
[37] S. Joucla and B. Yvert. The "mirror" estimate: an intuitive predictor of membrane polarization during extracellular stimulation. Biophysical journal, 96(9):34953508, May 2009.

[38] C. H. See, R. A. Abd-Alhameed, and P. S. Excell. Computation of Electromagnetic Fields in Assemblages of Biological Cells Using a Modified Finite-Difference Time-Domain Scheme. Microwave Theory and Techniques, IEEE Transactions on, 55(9):1986-1994, 2007.

[39] Gorazd Pucihar, Damijan Miklavcic, and Tadej Kotnik. A time-dependent numerical model of transmembrane voltage inducement and electroporation of irregularly shaped cells. IEEE transactions on bio-medical engineering, 56(5):1491-1501, May 2009.

[40] L. J. Leon and F. A. Roberge. A model study of extracellular stimulation of cardiac cells. Biomedical Engineering, IEEE Transactions on, 40(12):1307-1319, December 1993.

[41] K. R. Foster and A. E. Sowers. Dielectrophoretic forces and potentials induced on pairs of cells in an electric field. Biophysical journal, 69(3):777-784, September 1995 .

[42] Konstantinos Xylouris, Gillian Queisser, and Gabriel Wittum. A three-dimensional mathematical model of active signal processing in axons. Comput. Vis. Sci., 13(8):409-418, December 2010.

[43] E. J. Vigmond, R. Weber dos Santos, A. J. Prassl, M. Deo, and G. Plank. Solvers for the cardiac bidomain equations. Progress in biophysics and molecular biology, 96(1-3):3-18, January 2008.

[44] Joe Pitt-Francis, Pras Pathmanathan, Miguel O. Bernabeu, Rafel Bordas, Jonathan Cooper, Alexander G. Fletcher, Gary R. Mirams, Philip Murray, James M. Osborne, and Alex Walter. Chaste: A testdriven approach to software development for biological modelling. Computer Physics Communications, 180(12):2452-2471, December 2009.

[45] Robert Plonsey and Dennis Heppner. Considerations of quasi-stationarity in electrophysiological systems. Bulletin of Mathematical Biology, 29(4):657-664, December 1967.

[46] K. Lindsay. From Maxwell's equations to the cable equation and beyond. Progress in Biophysics and Molecular Biology, 85(1):71-116, May 2004.

[47] B. P. Lamichhane and B. I. Wohlmuth. Mortar finite elements for interface problems. Computing, 72:333348, May 2004.

[48] C. S. Henriquez. Simulating the electrical behavior of cardiac tissue using the bidomain model. Critical reviews in biomedical engineering, 21(1):1-77, 1993.

[49] Christophe Geuzaine and Jean-François Remacle. Gmsh: A 3-D finite element mesh generator with builtin pre- and post-processing facilities. Int. J. Numer. Meth. Engng., 79(11):1309-1331, 2009.
[50] M. L. Hines and N. T. Carnevale. The NEURON Simulation Environment. Neural Computation, 9(6):11791209, August 1997.

[51] Assaf Rotem and Elisha Moses. Magnetic stimulation of one-dimensional neuronal cultures. Biophysical journal, 94(12):5065-5078, June 2008.

[52] Greg Stuart and Nelson Spruston. Determinants of Voltage Attenuation in Neocortical Pyramidal Neuron Dendrites. The Journal of Neuroscience, 18(10):35013510, May 1998.

[53] Tadej Kotnik, Damijan Miklavčič, and Tomaž Slivnik. Time course of transmembrane voltage induced by time-varying electric fields - a method for theoretical analysis and its application. Bioelectrochemistry and Bioenergetics, 45(1):3-16, March 1998.

[54] W. Krassowska and J. C. Neu. Response of a single cell to an external electric field. Biophys $J, 66(6): 1768$ 1776, June 1994.

[55] Eva Syková and Charles Nicholson. Diffusion in Brain Extracellular Space. Physiological Reviews, 88(4):12771340, October 2008.

[56] Henrik Lindén, Tom Tetzlaff, Tobias C. Potjans, Klas H. Pettersen, Sonja Grün, Markus Diesmann, and Gaute T. Einevoll. Modeling the Spatial Reach of the LFP. Neuron, 72(5):859-872, December 2011.

[57] S. Vassanelli and P. Fromherz. Transistor records of excitable neurons from rat brain. Applied Physics A: Materials Science $\&$ Processing, V66(4):459-463, April 1998.

[58] Z. F. Mainen, J. Joerges, J. R. Huguenard, and T. J. Sejnowski. A model of spike initiation in neocortical pyramidal neurons. Neuron, 15(6):1427-1439, December 1995 .

[59] Jan Reinoud R. Buitenweg, Wim L. Rutten, and Enrico Marani. Geometry-based finite-element modeling of the electrical contact between a cultured neuron and a microelectrode. IEEE transactions on bio-medical engineering, 50(4):501-509, April 2003.

[60] Henry C. Tuckwell. Introduction to Theoretical Neurobiology: Volume 2, Nonlinear and Stochastic Theories, volume 2. Cambridge University Press, April 1988.

[61] Andrea Marcantoni, Valentina Carabelli, Valentina Comunanza, Daniela Gavello, Jonathan R. Ruiz, and Emilio Carbone. Microelectrode array (MEA) analysis of ion channels controlling spontaneous Chromaffin cells electrical activity. In 6th International Meeting on SubstrateIntegrated Micro Electrode Arrays, 2008. 


\section{Supplementary Material}

\section{Stability of the Numerical Scheme}

The explicit Euler form of the problem, although easily implementable, is only conditionally stable. As presented in section 2.3, a Crank-Nicolson (CN) scheme can be adapted to the solving algorithm providing excellent results (Fig. 3 and 4). In this section, the strict stability requirements for the Euler scheme and the more flexible $\mathrm{CN}$ requirements are shown.

Stability analysis for the general discretized version of the problem (Eqs. (14),(15),(20) and (21)) is complex for two reasons: first, the piecewise nature of the problem resulting from the membrane, and second, the separation of the space and time equations in two steps. This results in a situation, where actually the difference between potentials, the membrane voltage, is the relevant parameter on the membrane while in the classic stability analysis of the diffusion equation it is the potential itself. The membrane is the place where local source terms, the membrane currents, are much more likely to cause instabilities than is the solution of the Laplace or Poisson equation. Therefore we restrict our study to this area and neglect possible variations in potentials of the non-membrane nodes.

The problem was analyzed for a regular $2 \mathrm{D}$ triangulation of typical element length $h$ (Fig. S.1(A)). At the membrane interface, an expression for $I_{m}$ can be obtained and replaced in (20) and (21). Beginning with (14), for a membrane node $x^{\mathrm{j}}$, assuming no external current sources, and $u^{\mathrm{j}}=1$ one has,

$$
\sigma \sum_{\mathrm{k}} \Phi^{\mathrm{k}} \int_{\Omega} \nabla v^{\mathrm{k}} \cdot \nabla v^{\mathrm{j}} d \mathbf{x}+I_{m}^{\mathrm{j}} \int_{\Gamma}\left[v^{\mathrm{j}}\right]_{i, e} d s=0
$$

Note that $u^{\mathrm{j}}=1$ is a common assumption in FEM implementations of the Neumann BC. This assumption was also used in the membrane test functions of the computer solver. The membrane current at node $x^{\mathrm{j}}$ can be calculated from either the extracellular or intracellular potentials. With the adjacent extracellular nodes, this value is:

$$
I_{m}^{\mathrm{j}}=-\sigma \frac{\sum_{\mathrm{k}} \Phi_{e}^{\mathrm{k}} \int_{\Omega} \nabla v^{\mathrm{k}} \cdot \nabla v^{\mathrm{i}} d \mathbf{x}}{-\int_{\Gamma} v^{\mathrm{i}} d s}
$$

In the two dimensional triangulation (Fig. S.1(A)), expansion and integration with the classic "hat" test functions in (24), produces for the extra- and intracellular part:

$$
\begin{aligned}
& I_{m}^{j}=\frac{\sigma}{h}\left(2 \Phi_{e}^{\mathrm{j}}-\frac{1}{2} \Phi_{e}^{\mathrm{j}-1}-\frac{1}{2} \Phi_{e}^{\mathrm{j}+1}-\Phi_{e}^{\mathrm{p}}\right), \\
& -I_{m}^{j}=\frac{\sigma}{h}\left(2 \Phi_{i}^{\mathrm{j}}-\frac{1}{2} \Phi_{i}^{\mathrm{j}-1}-\frac{1}{2} \Phi_{i}^{\mathrm{j}+1}-\Phi_{i}^{\mathrm{q}}\right) .
\end{aligned}
$$

Subtracting (26) from (25) and employing the definition of membrane voltage $V_{m}^{\mathrm{j}}=\Phi_{i}^{\mathrm{j}}-\Phi_{e}^{\mathrm{j}}$ one obtains:

$$
I_{m}^{\mathrm{j}}=-\frac{\sigma}{h}\left(V_{m}^{\mathrm{j}}-\frac{1}{4} V_{m}^{\mathrm{j}-1}-\frac{1}{4} V_{m}^{\mathrm{j}+1}-\frac{1}{2} \Phi_{i}^{\mathrm{q}}+\frac{1}{2} \Phi_{e}^{\mathrm{p}}\right)(
$$

As mentioned above at this point the fluctuations of the exterior values $\Phi_{i}^{\mathrm{q}}, \Phi_{e}^{\mathrm{p}}$ around the "true" values are ignored.
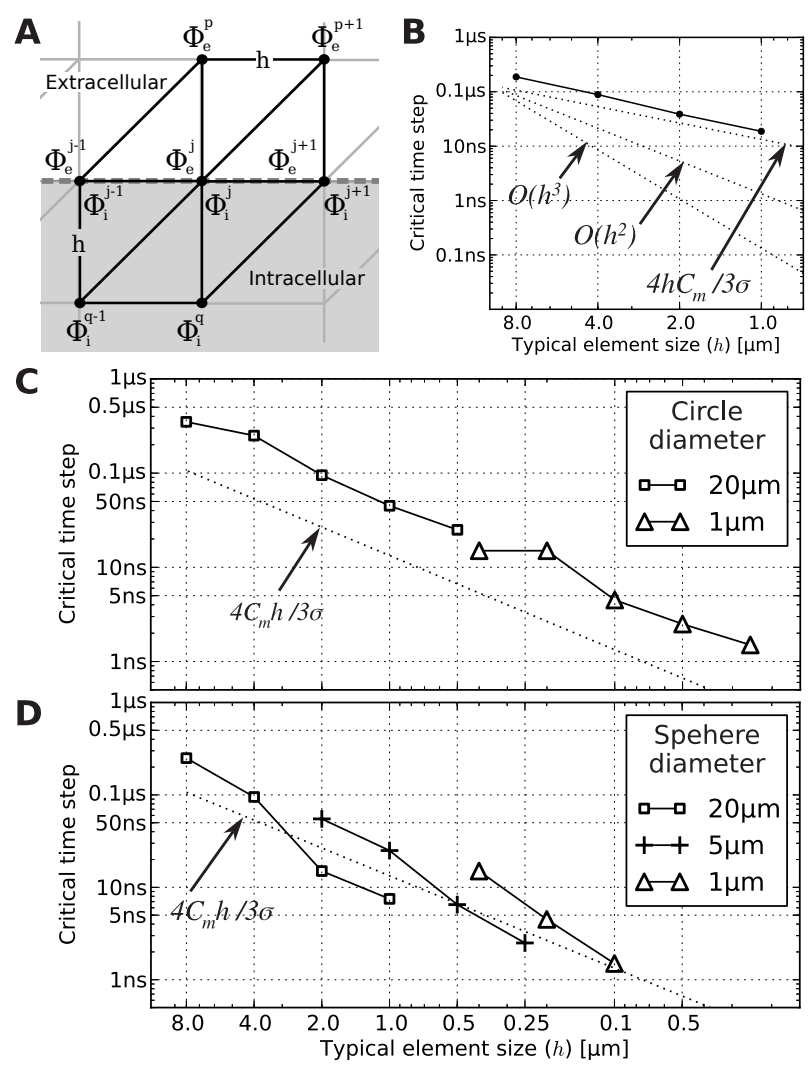

Figure S.1: Time step restrictions depending on the typical element size $h$ for $2 \mathrm{D}$ and $3 \mathrm{D}$ meshes with the basic Euler scheme. (A) Notation for a section of the regular $2 \mathrm{D}$ triangulation used to find the stability conditions. The dashed horizontal line represents the membrane and the gray area the intracellular space. Index j goes along an "infinite" membrane while $\mathrm{p}$ and $\mathrm{q}$ are the nearest extra and intracellular nodes to node j. (B) Experimental numerical results showing the critical time step for a $16 \mu \mathrm{m}$ cubic cell under a homogeneous electric field as the mesh typical element decreases. The field was oriented normal to one of the faces, parameters are identical to the ones used in Fig. 5. Above the solid line the numerical solution was unstable. The cell was defined by regular tetrahedrons of different heights $h=\{8,4,2,1\} \mu \mathrm{m}$. The dotted lines represent the time step stability restriction approximation for a regular grid (30) and alternatively, how an order $h^{2}$ and $h^{3}$ dependence would look like. Stability in 3D shows a clear $h$ dependence. (C) Numerical results showing the critical time step for the simulation of a $d=20 \mu \mathrm{m}$ and a $d=1 \mu \mathrm{m}$ circular cells as the typical element decreases. Parameters were identical to Fig. 5. The cells meshes consisted of irregular triangles generated with Gmsh's MeshAdapt algorithm. Dotted lines represent the time-step limit approximation for a regular grid (Eq. (30)). Stability in 2D also shows a clear $h$ dependence. (D) Same than (C) but for three 3D spherical cells with diameters $20 \mu \mathrm{m}$, $5 \mu \mathrm{m}$ and $1 \mu \mathrm{m}$. The cells were formed by irregular tetrahedrons generated with Gmsh's Delaunay 3D algorithm. Although the parameter $h$ was conserved for the majority of tetrahedrons, statistical analysis of showed some of them had much shorter edges than the value specified by the $h$ parameter. This explains the variations of the the 20 and 5 $\mu \mathrm{m}$ traces. Depending on the tools used for meshing, the quality of the mesh in $3 \mathrm{D}$ is more critical for stability. 
In this case the standard ansatz of the von Neumann stability analysis can be used: a linear combination of periodic solutions of the form,

$$
V_{m}^{\mathrm{j}, \mathrm{n}}=\xi^{\mathrm{n}} e^{\mathrm{i} \gamma \mathrm{j} h} .
$$

With $\mathrm{j}, \mathrm{n}$ the space and time indexes, $\mathrm{i}$ the imaginary unit, and $\gamma$ a variable wave number. The starting condition for $V_{m}$ is on average zero. This implies potentials in the extra- and intracellular region of a region of membrane are on average the same. For simplicity we choose this value to be zero. Under this conditions potentials are allowed to fluctuate but only at larger scales than the fluctuations of $V_{m}$. Inserting (27) in the Euler scheme (20) and using a passive membrane resistance produces:

$$
\begin{aligned}
V_{m}^{\mathrm{j}, \mathrm{n}+1} & =(1-\alpha-\beta) V_{m}^{\mathrm{j}, \mathrm{n}}+\frac{\beta}{4}\left(V_{m}^{\mathrm{j}-1, \mathrm{n}}+V_{m}^{\mathrm{j}+1, \mathrm{n}}\right) \\
\alpha & =\frac{\Delta t}{C_{m}} \frac{1}{R_{m}}, \quad \beta=\frac{\sigma \Delta t}{C_{m} h} .
\end{aligned}
$$

Replacing (28) in (29) leads to the amplification factor:

$$
\xi_{E}=1-\alpha-\frac{\beta}{2}-\beta \sin ^{2} \frac{k h}{2} .
$$

Stability is obtained under the condition $\left|\xi_{E}\right| \leq 1$, which is true for

$$
\Delta t \leq \frac{2}{\frac{1}{R_{m} C_{m}}+\frac{3}{2} \frac{\sigma}{C_{m} h}} .
$$

In cell biology it is usual that $C_{m} h / \sigma \ll R_{m} C_{m}$ so the condition can be approximated by:

$$
\Delta t<\frac{4 C_{m} h}{3 \sigma} .
$$

This was tested in numerical experiments in 2D and 3D (Figure $\mathrm{S} .1(\mathrm{~B}, \mathrm{C}, \mathrm{D}))$. The factor $4 / 3$ is not exact as we dropped the contribution of the exterior values $\Phi_{i}^{\mathrm{q}}, \Phi_{e}^{\mathrm{p}}$. Their influence should be on the order of the other potential-difference terms in 27 so that the result presented here is of the correct order.

The restriction on the time-step is laxer in the CrankNicolson scheme. Inserting (27) in the CN scheme (21) expands to:

$$
\begin{gathered}
\left(1+\frac{\beta}{2}\right) V_{m}^{\mathrm{j}, \mathrm{n}+1}-\frac{\beta}{8}\left(V_{m}^{\mathrm{j}-1, \mathrm{n}+1}+V_{m}^{\mathrm{j}+1, \mathrm{n}+1}\right)= \\
\left(1-\frac{\beta}{2}-\alpha\right) V_{m}^{\mathrm{j}, \mathrm{n}}+\frac{\beta}{8}\left(V_{m}^{\mathrm{j}-1, \mathrm{n}}+V_{m}^{\mathrm{j}+1, \mathrm{n}}\right)
\end{gathered}
$$

Use of (28) produces the amplification factor:

$$
\xi_{C N}=\frac{1-\frac{\beta}{2}\left(\frac{1}{2}+\sin ^{2} \frac{\gamma h}{2}\right)-\alpha}{1+\frac{\beta}{2}\left(\frac{1}{2}+\sin ^{2} \frac{\gamma h}{2}\right)} .
$$

Adding $-1+\frac{\beta}{2}\left(\frac{1}{2}+\sin ^{2} \frac{\gamma h}{2}\right)$ to the inequality: $-1 \leq$ $\xi_{C N} \leq 1$, gives:

$$
-\beta\left(\frac{1}{2}+\sin ^{2} \frac{\gamma h}{2}\right) \leq \alpha \leq 2
$$

$\alpha$ and $\beta$ are always positive so the left inequality is always true. The right part establishes that the stability limits are set by the millisecond scale membrane time constant $\tau_{m}=R_{m} C_{m}$ :

$$
\Delta t \leq 2 \tau_{m}
$$

\begin{tabular}{|c|c|c|c|}
\hline Name & Definition & Unit & Order \\
\hline$\Phi_{e, i}$ & Extrac./Intrac. potential & $\mathrm{mV}$ & $10^{0}-10^{1}$ \\
\hline$V_{m}$ & Membrane voltage & $\mathrm{mV}$ & $10^{1}-10^{2}$ \\
\hline$I_{m}$ & Current towards the membrane & $\mu \mathrm{A} / \mathrm{cm}^{2}$ & $10^{1}-10^{4}$ \\
\hline$I_{\text {ion }}$ & Trans-membrane ionic current & $\mu \mathrm{A} / \mathrm{cm}^{2}$ & $10^{1}$ \\
\hline$\sigma_{e, i}$ & Extrac./Intrac. conductivity & $\mathrm{mS} / \mathrm{cm}^{1}$ & $10^{1}$ \\
\hline$C_{m}$ & Membrane specific capacitance & $\mu \mathrm{F} / \mathrm{cm}^{2}$ & $10^{0}$ \\
\hline$R_{m}$ & Membrane specific resistance & $\Omega \mathrm{cm}^{2}$ & $10^{3}-10^{4}$ \\
\hline$d$ & Cell diameter & $\mathrm{cm}$ & $10^{-3}$ \\
\hline $\mathbf{E}$ & Electric field & $\mathrm{mV} / \mathrm{cm}$ & $10^{0}-10^{4}$ \\
\hline
\end{tabular}

Table S.1: List of the main variables and constants of the model, units used, and rough orders of magnitude.

\begin{tabular}{|c|c|c|c|c|}
\hline$\Delta t$ & $h$ & Method & Duration & NRMSD \\
\hline \multirow{3}{*}{$50 \mathrm{~ns}$} & $1 \mu \mathrm{m}$ & Eul. & $5.78 \mathrm{~s}$ & $4.40 \%$ \\
\cline { 2 - 5 } & $1 \mu \mathrm{m}$ & $\mathrm{CN}$ & $11.17 \mathrm{~s}$ & $6.04 \%$ \\
\cline { 2 - 5 } & $1 \mu \mathrm{m}$ & $\mathrm{ECN}$ & $11.88 \mathrm{~s}$ & $0.29 \%$ \\
\hline \multirow{3}{*}{$5 \mathrm{~ns}$} & $0.5 \mu \mathrm{m}$ & $\mathrm{Eul}$ & $228.57 \mathrm{~s}$ & $0.31 \%$ \\
\cline { 2 - 5 } & $0.5 \mu \mathrm{m}$ & $\mathrm{CN}$ & $591.67 \mathrm{~s}$ & $0.68 \%$ \\
\cline { 2 - 5 } & $0.5 \mu \mathrm{m}$ & $\mathrm{ECN}$ & $610.22 \mathrm{~s}$ & $0.15 \%$ \\
\hline \multirow{3}{*}{$0.5 \mathrm{~ns}$} & $0.25 \mu \mathrm{m}$ & $\mathrm{Eul}$. & $11053.0 \mathrm{~s}$ & $0.05 \%$ \\
\cline { 2 - 5 } & $0.25 \mu \mathrm{m}$ & $\mathrm{CN}$ & $24414.1 \mathrm{~s}$ & $0.08 \%$ \\
\cline { 2 - 5 } & $0.25 \mu \mathrm{m}$ & $\mathrm{ECN}$ & $26267.7 \mathrm{~s}$ & $0.12 \%$ \\
\hline
\end{tabular}

Table S.2: Complete results with the three methods (Euler, CrankNicolson $(\mathrm{CN})$ and Euler-Crank-Nicolson (ECN)) for the convergence experiment in Fig. 3(A,B) with the reference software. The table shows the time step used, the typical element size $(h)$, the method, computation duration in seconds, and the normalized root mean square deviation to the analytic solution. The extra duration of the $\mathrm{CN}$ is explained by the extra restriction imposed to the solver to satisfy values of $\mathbf{I}_{m}^{\mathrm{n}+1}$. Although ECN solves two linear systems at each time-step, the durations are similar compared to CN. This is explained by the second solution of the linear system using the potential calculated in the first solution as the initial guess in the generalized minimal residual method.

\section{D Cell Analytic Solution}

The analytic solution for the homogeneous stimulus of a $2 \mathrm{D}$ cell was used to compute the reference solution in Figures 2 and3. The solution is known from $[54,15]$. Potentials for a circular cell of diameter $d$ under a homogeneous step field $E$ starting at $t=0$ are,

$$
\begin{aligned}
\Phi_{i}(r, \theta, t) & =-a(t) \cdot E r \cos \theta r<d / 2 \\
\Phi_{e}(r, \theta, t) & =-E r \cos \theta-b(t) \cdot E \frac{d^{2}}{4 r} \cos \theta r>d / 2 \\
V_{m}(\theta, t) & =E d \cos \theta\left(1-e^{-\frac{t}{\tau}}\right)(1-\epsilon)
\end{aligned}
$$

With,

$$
\begin{aligned}
a(t) & =\frac{2 \sigma_{e}}{\sigma_{i}+\sigma_{e}}\left\{e^{-\frac{t}{\tau}}+\left(1-e^{-\frac{t}{\tau}}\right) \epsilon\right\} \\
b(t) & =1-\frac{2 \sigma_{i}}{\sigma_{i}+\sigma_{e}}\left\{e^{-\frac{t}{\tau}}+\left(1-e^{-\frac{t}{\tau}}\right) \epsilon\right\} \\
\tau & =\frac{1}{\frac{1}{C_{m} R_{m}}+\frac{2 \sigma_{i} \sigma_{e}}{C_{m} d\left(\sigma_{i}+\sigma_{e}\right)}}, \epsilon=\frac{\tau}{C_{m} R_{m}}
\end{aligned}
$$


122 CHAPTER 7. FAST SIMULATION OF ACTIVITY AT CELL MEMBRANES 


\section{Discussion and Outlook}

In this work a numerical method and a software package to treat the electrical activity of three-dimensional neurons and their extracellular space were presented. Modern neuroscience requires new tools that explicitly consider the extracellular space and the potentials on it. The tool described has been analytically and numerically validated and has already provided insight into the activity of the cell during extracellular stimulation. This section summarizes the main results from this work, discusses their contribution and proposes future work.

Our quantitative understanding of electrophysiology is based on the concepts of the Hodgkin-Huxley model and the cable equation [3]. These concepts describe the behavior of isolated cells inside an homogeneous isopotential space. Extracellular potentials can be added to this picture with the activating function [34] or the line source approximation [36]. However there are fundamental limitations to the validity of these approaches for example on structures that are not thin, such as the soma (Chapter 7 Figure 5). The intrinsic feedback between endogenous extracellular fields and cellular activity is never captured by these traditional approaches, as always only one direction, e.g. membrane currents to potentials, is captured. When the physical problem is formulated to take the interaction between currents and fields into account (Chapter 2) partial differential equations (PDE) emerge (Section 2.5). While a few modeling works have offered alternatives to treat these equations, these have been limited to unrealistic spatial scales [38, 53], restrictive time evolution schemes [35, 37, 38, 53, 52], two dimensions [37], and the absence of ion channels [35, 37, 38, 52].

\subsection{The solution method}

A new numerical method for the treatment of these equations was presented. The method allows detailed simulations of the electric activity of excitable cells and their interaction with extracellular potentials. Membranes in interaction with the potentials are explicitly modeled (Chapter 2). These membranes can have arbitrary shapes on realistic sub-micrometer scales (Chapter 7,3.3,3.5) and contain voltage dependent conductances (Section 2.7.1 and Chapter 7). The extracellular space is an integral part of the mathematical structure of this method (Section 2.4). The method is able to solve problems with mixed boundary conditions, represent geometric details, and allows relatively long simulated times in much shorter computation times than previously possible (Chapter 7, Sections 3.1-3.5).

Construction of the numerical method required understanding the electrodynamic 
principles behind neuronal activity. This work started with a review of the fundamentals behind the neuronal electric problem in Chapter 2. The quasi-static approximation and the volume conductor were presented in Section2.3 and 2.4. The equations that describe the general electrical activity of a three-dimensional neuron: the space $/ \mathrm{mem}$ brane equations $(\mathrm{S} / \mathrm{M})$, were presented in a single framework in Section 2.5. In Chapter 3 analytical solutions to these equations were given.

In Chapter 4 a discretization procedure based on the separation of the spatial an time domain in the $\mathrm{S} / \mathrm{M}$ equations was presented. This solution strategy extended from the quasi-static approximation and was detailed in Section 4.3. The closest treatment of this problem was the work of Ying and Henriquez [37] but their description of the solution was not detailed and their numerical integration scheme was limited (Section 5.6). The separation of the spatial and time domain, facilitate the numerical solution proposed in the same chapter. At the end of Chapter 4 two numerical integration schemes: the forward Euler and the Crank-Nicolson $(\mathrm{CN})$ method were introduced in sections 4.5 and 4.5.2. Although the CN method is implicit and adds an unknown, it was shown that the separation of space and time allowed its implementation in a simple matrix structure snot different from the Euler method.

In Chapter 5 the discretization scheme was validated. Numerical stability of the method was approached using analytically tractable problems. The basic stability criterion turned out to be linearly related to the typical grid spacing $h$. From the theoretical point of view this result was unexpected. An $h^{2}$ dependence exists in the diffusion equation[104], the propagation of action potential [47, 129] and the heart bidomain [54]. It is believed that this linear dependence on $h$ is a consequence of the separation of the time and space equations and the dimensionality of the problem: only the component of potential gradients that is oriented perpendicular to the membrane contributes to the membrane current $I_{m}$. However further work needs to be done to assert this claim. From the numerical solution point of view an $h$ dependence reduces restrictions in the meshing schemes. Experimentally it was shown that this linear term extended to three-dimensions.

Stability analysis also allowed to demonstrate the superiority of the CN scheme over the forward Euler Method in chapter Chapter 5. Replacing the Euler forward solver by the implicit and numerically more stable CN solver, it was possible to increase the simulation time steps and thereby reduce computation time by orders of magnitude. For the CN solver the time step can approach the membrane time constant while for the Euler, the much shorter cell time constant primes (Section 5.6). This finding is fundamental for long time simulations where the main observed phenomena lie mainly in the membrane time constant regime. The $\mathrm{CN}$ solver has been used in other solutions of the cable equation [107].

The CN method is however known for its spurious oscillations [12]. Those oscillations have been observed in the solution of the membrane current $I_{m}$ during simulation. Although these oscillations compensate and reduce the error, to obtain more reliable extracellular potential values, improvements should be done. Backward Euler methods have been used in cable solvers but its accuracy is lower than that of the CN's [115]. A more robust alternative can be found in adaptive time step and higher order methods 
such as the ones provided by CVODE [130]. These have proven successful in cable simulators.

The presented method has further limitations. The dependence of the CN stability in the passive membrane time constant is determined by the ionic current term. Experimental results with active channels have shown that fast dynamics such as the ones from sodium demand for shorter time steps. The relation between the active dynamics and the CN solution scheme should be studied. Alternatives exist. Heart bidomain solvers for example, integrate the ionic currents with time steps shorter than the ones used for the spatial solution[131]. Whether this alternative is applicable to the $\mathrm{S} / \mathrm{M}$ solvers should be determined. The use of adaptive time steps for the treatment of ionic currents combined with Hines method (Section 4.6) is the alternative taken by cable solvers.

\subsection{The software implementation}

The solver of the S/M equations presented in this work was named CHASTE-Membrane (CM). Previous solvers for the $\mathrm{S} / \mathrm{M}$ equations used closed source $[37,38,53]$ and commercial software [35, 39, 52]. CM was released as an open source to the scientific community and builds upon the open source initiative CHASTE [117]. Along with CHASTE, other modern, mainstream high performance and parallel, open computing libraries were used, including PETSc (Argonne National Lab.), MPI (Message Passing Interface standard), HDF5 (NCSA and others), VTK (Kitware Inc.) and CellML (Europe's Virtual Physiological Human Project). The use of open and well supported libraries ensures their continued development and optimization. CellML ${ }^{1}$ grants access to a public library of ion channel kinetic definitions and eases the implementation of new ion channel models.

Standard object oriented programming techniques were used during the development of the tool. The use these techniques is an important software practice as other developers can more easily access the code. Finite element solvers are complex software pieces that have to deal with a variety of discretization formats and platforms while offering high performance and an accessible user interface. The fact that CHASTE used object oriented techniques was one of the principal reasons for its choice and this facilitated enormously the development process (Chapter 6).

CHASTE-Membrane version 1.0 was developed during 21 months and at the moment can produce results with very high performance. The $\mathrm{CN}$ was fundamental for this achievement as it allowed to run simulations in minutes that otherwise would have taken weeks with the Euler (Chapter 7, Section 3.3). With CM it is now possible to model multiple cells and these cells can alter their activity through the extracellular space (Chapter 7, Section 3.4). In Chapter 7 Section 3.5 it was verified that realistic setups can be modeled. In the examples presented in Chapter 7 possible applications were presented. The examples focused on the effects of crammed extracellular space and the interaction between extracellular fields and neuronal activity on realistic spatial scales and with realistic parameters. Numerous other problems can be addressed by

\footnotetext{
${ }^{1}$ http://www.cellml.org/
} 
CM. Starting with small geometries as the ones needed to study depolarization of spines, all the way to very large systems like the cross-talk of local field potentials and electrical activity of hundreds of tightly packed cells. With these characteristics, the tool is ready to study ephaptic interaction $[10,9,8]$.

Going beyond the possibilities of cable tools (e.g. NEURON[12] or GENESIS[107]), the finite element approach in CM allows the implementation of other physical elements important for neural recording and stimulation. These include extraneous elements such as glass pipettes or recording electrodes. Heterogeneous distribution or properties of ion channels can be implemented on a triangle by triangle basis, without imposing a radial symmetry. These are important features, necessary to understand for instance, the heterogeneity of extracellularly recorded action potentials [132, 133]. The most powerful feature of $\mathrm{CM}$ over cable equation solvers is the explicit definition of the extracellular space. This allows a direct examination of effects like ephaptic coupling, not only on large scales but also for directly opposing membranes. This also allows studying the effect of heterogeneous conductivity and anisotropies, which is where the line source approach breaks down.

Despite the large improvements with respect to time step and computation time, finite element simulations do not have the potential to fully replace tools such as $N E U$ $R O N$ or GENESIS. This is particularly true for problems that are insensitive to the limited conductance of the extracellular space or the detailed geometry of dendrites. Still, with mounting evidence for the synchronization of action potentials by extracellular potentials $[10,9,8]$ and the progress in neuro-stimulation techniques, many questions arise that cannot be comprehensively addressed under the assumptions of the cable equation. In this area, the presented method can enhance the understanding of neural activity, stimulation, and recording of neurons.

\subsubsection{Further improvement of the tool}

Several steps have already been taken to shorten the computation time further. In Chapter 7 , Section 3.3 a possible adaptive time stepping strategy was proposed using the membrane currents as the adaptive criterion. The software structure of CM already allows this run-time alteration of the time step. Simulation of intrinsic neuronal activity will greatly benefit from adaptive schemes combined with the CN solver because currents and electric fields change on timescales much larger than the cell time constant.

This method was built from the beginning with parallelism in mind and the software framework supports the distributed solution of the linear system and distributed meshes. Initial tests have been performed and the most important aspect now is the development of better partition algorithms for the main mesh. The partitioning algorithm should determine whether to partition a given cell membrane (see as an example Figure 8.1) or to keep as much of possible of the cell in a given parallel machine. The greatest benefits from parallelization will obviously come from distribution of large meshes as time evolution in the $\mathrm{S} / \mathrm{M}$ problem is tightly coupled. 


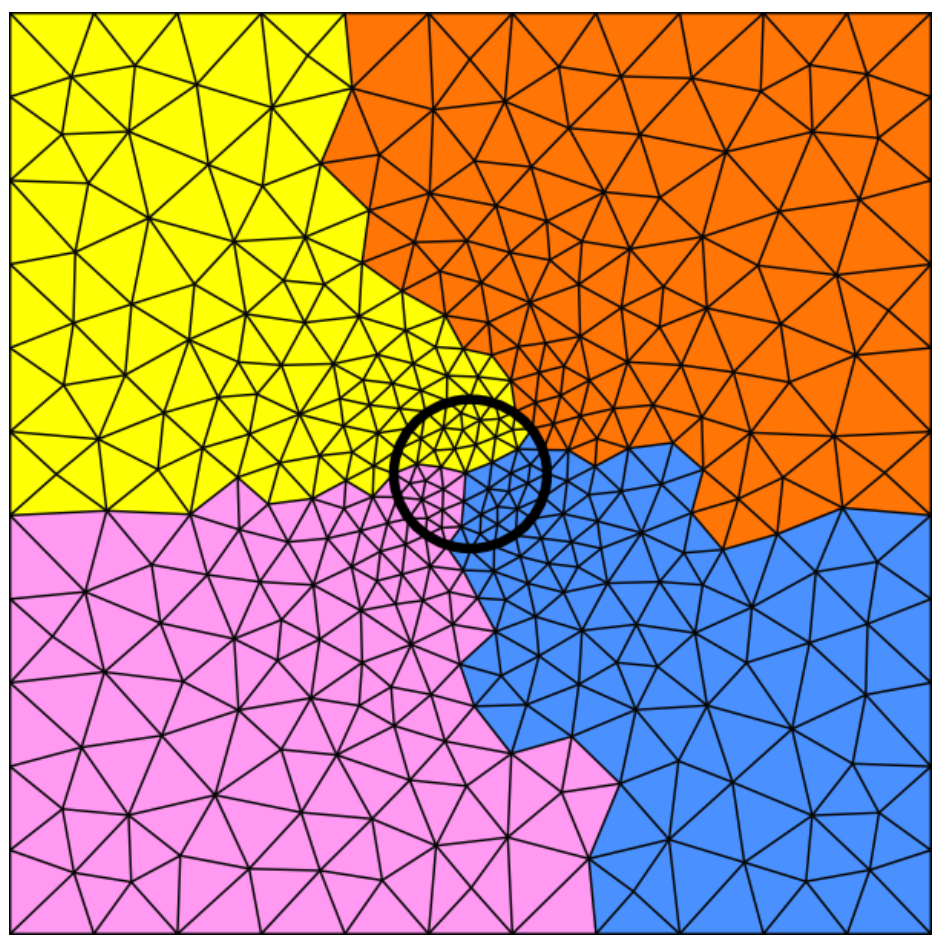

Figure 8.1: Example of a mesh in which the cell (black circle) was partitioned through. Colors represent different partitions of the mesh. Alternatively, the cell could have been assigned to a color single region. What would be the best strategy for parallelization? Is communication within the parallel nodes significantly increased when they share a cell? 


\section{A Neuron electrophysiology parameters}

This chapter provides a summary and comparison of basic neuronal modeling parameters obtained from the literature. For the summary, experimental and theoretical studies were used. The chapter also provides example values and orders of magnitude of some typical variables in neural electrophysiology. For the non trained in physics or electrophysiology this summary provides a useful additional guide to this work. Note that in the chapter the terms resistivity and resistance, which are usually mixed up in neuroscience works, are treated as two separate concepts. An excellent guide on the naming of these parameters and in general to theoretical electrophysiology is provided by Plonsey and Barr's book [47].

\section{A.1 Summary of passive parameters}

Tables A.1 and A.2 list values for the classic specific intracellular $\left(R_{i}\right)$ and extracellular $\left(R_{e}\right)$ resistivity, specific membrane resistance $\left(R_{m}\right)$ and specific membrane capacitance $\left(C_{m}\right)$ and values of the time and length constants (see also Section 2.6.1). Figure A.1 illustrates the relation between the length constant and intracellular resistivity, membrane resistance and diameter. It is well known that the intracellular resistivity is on the order of $100 \Omega \cdot \mathrm{cm}$. For three typical intracellular resistivities $(50,100,200$ $\Omega \cdot \mathrm{cm}$ ) and two diameters 1 and $10 \mu \mathrm{m}$, the length constant is shown.

\section{A.2 Summary of active parameters}

Table A.3 compares the channel conductive densities in five influential neurophysiology studies. The values in the last column where used in Chapter 7 for the 3D neuronal model. 


\begin{tabular}{|c|c|c|c|c|}
\hline Passive parameters & $R_{i}[\Omega \cdot \mathrm{cm}]$ & $R_{e}[\Omega \cdot \mathrm{cm}]$ & $R_{m}\left[\Omega \cdot \mathrm{cm}^{2}\right]$ & $C_{m}\left[\frac{\mu F}{c m^{2}}\right]$ \\
\hline Dayan and Abbot [67] & 100 & & $10 \mathrm{k}$ & \\
\hline NEURON default [115] & 35.4 & & 1000 & 1 \\
\hline Rottem et al. dendrite [73] & 83.53 & & 5000 & 1 \\
\hline Rottem et al. axon [73] & 5.09 & & 300 & 1 \\
\hline Meyer et al. (dendrite, hippoc. culture)[134] & 300 & & $40 \mathrm{k}$ & 1 \\
\hline Bronzino [135] & $50-400$ & & $1000-5000$ & $1-2$ \\
\hline McIntyre et al. Dend [46] & 300 & 350 & & 1 \\
\hline Axon (myelinated) [46] & 70 & 350 & & 2 \\
\hline Ranck [136] & & $233-357$ & & \\
\hline Nunez (CSF) [62] & & 64.1 & & \\
\hline Bedard et al. [33] & & 64.1 & $\mathrm{HH}$ & 1 \\
\hline Mainen and Sejnowski [137] & 150 & & $30 \mathrm{k}, \mathrm{HH}$ & 0.75 \\
\hline Myelinated [137] & & & 50 (ax. nodes) & 0.02 \\
\hline Gold et al. $\left(R_{e}\right.$ of $\left.260-290\right)$ [40] & 70 & $640,260,290$ & 15000 & 1 \\
\hline Tuch et al. (CSF, 1.54-1.79 S/m) [138] & & 55.9 or 64.9 & & \\
\hline Jefferys [139] & & $<200$ & & \\
\hline Monai et al. [74] & 200 & 20 & $30 \mathrm{k}$ & 1.5 \\
\hline Ying and Henriquez [37] & 200 & 50 & 1000 & 1 \\
\hline Tuckwell (10um dend., cat spinal) [69] & 70 & & 2500 & 2 \\
\hline Plonsey and Barr [140] & 200 & 30 & 1480 & 1 \\
\hline Thurbon et al. [141] & $52-484$ & & $7 \mathrm{k}-66 \mathrm{k}$ & 1 \\
\hline Spruston et al. [142] & $200-400$ & & & \\
\hline Oltedal et al. [143] & 130 & & $24 \mathrm{k}$ & 1.1 \\
\hline Major et al. (rat CA3 pyramidal, slices) [144] & $170-340$ & & $120 \mathrm{k}-200 \mathrm{k}$ & $0.7-0.8$ \\
\hline Trevelyan and Jack (rat pyram. visual, $\left.36^{\circ}\right)[145]$ & $140-170$ & & $40 \mathrm{k}$ & $0.78-0.94$ \\
\hline Rapp et al. (guineapig, purkinje, soma) [146] & 250 & & 500 & $1.5-2$ \\
\hline Rapp et al. (purkinje, dendrite) [146] & 250 & & $100 \mathrm{k}$ & $1.5-2$ \\
\hline Hille [147] & & & $1000-5000$ & \\
\hline CHASTE-Membrane default & 100 & 100 & 1000 & 1 \\
\hline
\end{tabular}

Table A.1: Literature review of passive neuronal parameters. 


\begin{tabular}{|c|c|c|c|}
\hline Study & M.A. & U.A. & Dendrite \\
\hline E. Moses /A. Rotem (2008, ax: $1 \mu \mathrm{m}$, de: $5 \mu \mathrm{m})$ [73] & & $384 \mu \mathrm{m} / 300 \mu \mathrm{s}$ & $865 \mu \mathrm{m} / 5 \mathrm{~ms}$ \\
\hline E. Moses (2012, ax: $1 \mu \mathrm{m}$, de: $5 \mu \mathrm{m})$ (Person. comm.) & & $400 \mu \mathrm{m} / 100 \mu \mathrm{s}$ & $850 \mu \mathrm{m} / 1 \mathrm{~ms}$ \\
\hline Tuckwell [69] (cat spinal) & & & $1000 \mu \mathrm{m} / 5 \mathrm{~ms}$ \\
\hline Tuckwell [148] (theoretical, mamm, ax: $13 \mu \mathrm{m})$ & $2.5 \mathrm{~mm} /$ & $350 \mu \mathrm{m} /$ & \\
\hline Thurbon et al. [141] (rat CA1, apical) & & & $1060 \mu \mathrm{m}(+/-400) / 7-66 \mathrm{~ms}$ \\
\hline Thurbon et al. [141] (rat CA1, basal) & & & $510 \mu \mathrm{m}(+/-260) / 7-66 \mathrm{~ms}$ \\
\hline Spruston et al. [142] & & & $/ 28-66 \mathrm{~ms}$ \\
\hline 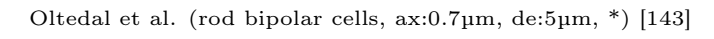 & & $572 \mu \mathrm{m} / 24 \mathrm{~ms}$ & $1.52 \mathrm{~mm} / 24 \mathrm{~ms}$ \\
\hline 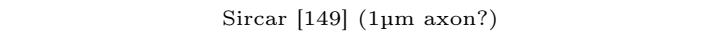 & $3 \mathrm{~mm} /$ & $500 \mu \mathrm{m} /$ & \\
\hline Major et al. (rat CA3 pyramidal, slices, $10 \mu \mathrm{m},{ }^{*}$ ) [144] & & & $3.96 \mathrm{~mm} /$ \\
\hline Major et al. (rat CA3 pyr., slices, $1 \mu \mathrm{m},{ }^{*}$ ) [144] & & & $1.25 \mathrm{~mm} /$ \\
\hline Rapp et al. (guineapig, purkinje, dendrite, *) [146] & & & $1.58 \mathrm{~mm} / 50 \mathrm{~ms}$ \\
\hline Meyer et al. (dendrite, hippoc. culture) [134] & & & $580 \mu \mathrm{m} /$ \\
\hline Nowak and Bullier $\left(\mathrm{Rm} 1000-5000 \mathrm{ohm}^{*} \mathrm{~cm} 2, R_{i}=150,{ }^{*}\right)[150]$ & & $129-289 \mu \mathrm{m} / \approx 200 \mu \mathrm{s}$ & \\
\hline Theoretical estimate (see figure A.1. $R_{i}$ approx. 150) & & $<1000 \mu \mathrm{m}$ & \\
\hline
\end{tabular}

Table A.2: Literature summary of the length/time constants for myelinated axons (MA), unmyelinated axons (UA) and dendrites. An $\left(^{*}\right)$ indicates that the values were not explicitly given in the paper but were calculated from the main paper result.

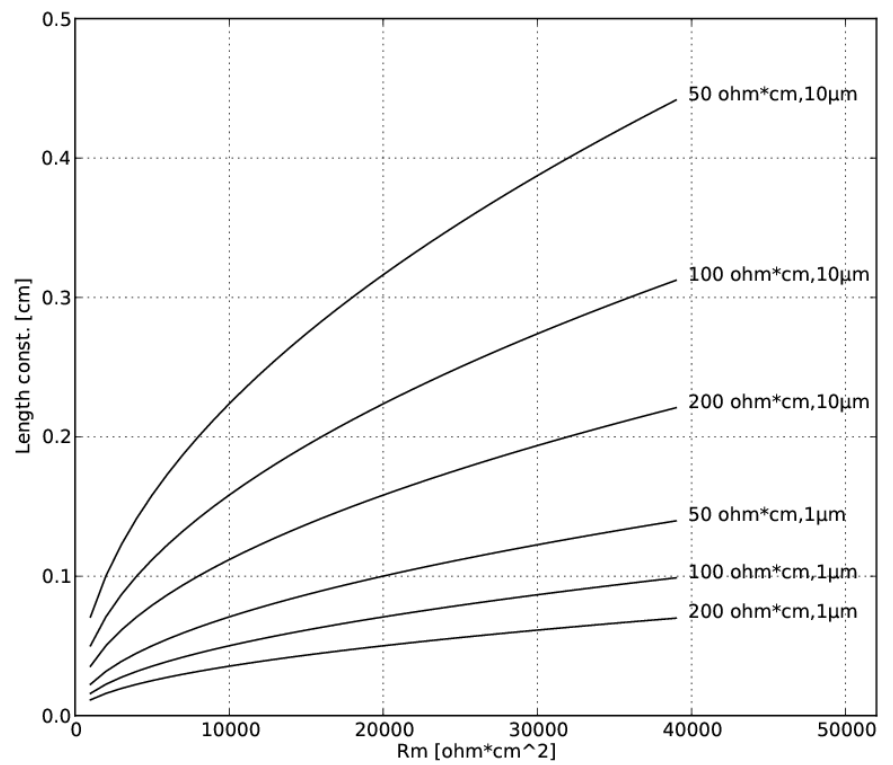

Figure A.1: Membrane resistance vs. length constant for three different values of intracellular resistivity $\left(50,100,200 \mathrm{Ohm}^{*} \mathrm{~cm}\right)$ and two different diameters $(1$ and 10 $\mu \mathrm{m})$. At about $150 \mathrm{Ohm}^{*} \mathrm{~cm}$ the length time constant of unmyelinated axons should be lower than $1 \mathrm{~mm}$ for realistic membrane resitances. 


\begin{tabular}{|c|c|c|c|c|c|c|}
\hline & {$[151]$} & {$[152]$} & {$[137]$} & {$[153]$} & {$[154]$} & 3D Models \\
\hline \multicolumn{7}{|l|}{ Sodium $(\mathrm{mS} / \mathrm{cm} 2)$} \\
\hline Reversal pot. (mV) & & 60 & 50 & & & \\
\hline Dendrite & 4.5 & 2 & 2 & $2-10$ & 2 & 10 \\
\hline Soma & 4.5 & $80(10-90$ tested $)$ & 2 & $75-80$ & 50 & 20 \\
\hline Hillock & $4.5-500$ & 400 & $3 \mathrm{~K}$ & 800 & & 20 \\
\hline AIS & 500 & $800(30-27 \mathrm{~K}$ tested $)$ & $3 \mathrm{~K}$ & 800 & 700 & 120 \\
\hline Axon (after AIS/rest) & $?$ & & - & & 50 & 20 \\
\hline Internodal & 8 & & - & 2 & & - \\
\hline Node & 250 & & $3 \mathrm{~K}$ & 500 & & - \\
\hline Potassium $(\mathrm{mS} / \mathrm{cm} 2)$ & Kv1 & & $\mathrm{Kv}$ & $\mathrm{Kv}$ & & \\
\hline Reversal pot. (mV) & & -85 & -90 & & & \\
\hline Dendrite & 10 & 20 & 0.01 & - & 0.3 & 10 \\
\hline Soma & 10 & 40 & 20 & 8 & 10 & 20 \\
\hline Hillock & $10-200$ & & 200 & 80 & & 20 \\
\hline AIS & 200 & $100(100-3 \mathrm{~K}$ tested $)$ & 200 & 80 & 200 & 30 \\
\hline Axon (after AIS/rest) & $?$ & & - & - & 40 & 20 \\
\hline Internodal & 10 & & - & - & & \\
\hline Node & - & & - & - & & \\
\hline \multicolumn{7}{|l|}{ Passive } \\
\hline Resistivity $(\mathrm{ohm} * \mathrm{~cm})$ & 100 & 150 & 150 & 150 & 80 & 100 \\
\hline Resistance $\left(\mathrm{ohm}^{*} \mathrm{~cm}^{2}\right)$ & $15 \mathrm{~K}$ & $50 \mathrm{~K}$ & $30 \mathrm{~K}$ & $30 \mathrm{~K}$ & $15 \mathrm{~K}$ & $20 \mathrm{~K}$ \\
\hline Capacitance $\left(\mathrm{uF} / \mathrm{cm}^{2}\right)$ & 0.9 & 1 & 0.75 & 0.75 & 1 & 1 \\
\hline$R_{m}$ internod $\left(\mathrm{ohm}^{*} \mathrm{~cm}^{2}\right)$ & & $2.5 \mathrm{M}$ & & - & $45 \mathrm{~K}$ & \\
\hline$R_{m}$ node $\left(\mathrm{ohm}^{*} \mathrm{~cm}^{2}\right)$ & & & 50 & 50 & & \\
\hline$C_{m}$ node $\left(\mathrm{uF} / \mathrm{cm}^{2}\right)$ & & 0.02 & 0.02 & 0.04 & 0.17 & \\
\hline
\end{tabular}

Table A.3: Channel densities in neurophysiology studies. The "3D models" column refers to the parameters used in Chapter 7. 


\begin{tabular}{|c|c|c|}
\hline Parameter & Dendrite & Axon \\
\hline \hline Diameter $(\mu m)$ & 5 & 1 \\
\hline Length $(\mu m)$ & 200 & 1000 \\
\hline$\lambda(\mu m)$ & 865 & 384 \\
\hline$\tau(m s)$ & 5 & 0.3 \\
\hline Axial spec. resist. $(\Omega \cdot \mathrm{cm})$ & 83.53 & 5.09 \\
\hline Memb. spec. resist. $\left(\Omega \cdot \mathrm{cm}^{2}\right)$ & 5000 & 300 \\
\hline
\end{tabular}

Table A.4: Rotem and Moses [73] cell parameters. A typical specific membrane capacitance of $C_{m}=1 \frac{\mu F}{c^{2}}$ was used in the paper.

\section{A.3 Example: calculating the cable equation parameters}

This section provides an example on calculating the membrane specific resistance $\left(R_{m}\right)$, the axial specific resistivity $\left(R_{i}\right)$, the membrane specific passive conductance $\left(g_{\text {pas }}\right)$ and the specific membrane capacitance $\left(C_{m}\right)$ from the time and length constants. On Section 2.6.1 only the equivalent terms $r_{m}, r_{i}$ and $c_{m}$ were used but often, the expression of the cable parameters in the former terms is required for tools like NEURON. The example values are presented together with the results in Table A.4.

For the dendrite $\tau=r_{m} c_{m}=R_{m} C_{m}$ and the membrane specific resistance should be,

$$
R_{m}=\frac{\tau}{C_{m}}=\frac{0.005[\mathrm{~s}]}{0.000001\left[\frac{\mathrm{F}}{\mathrm{cm}^{2}}\right]}=5000\left[\Omega \mathrm{cm}^{2}\right] .
$$

For tools like NEURON $R_{m}=\frac{1}{g_{\text {pas }}}$ and then $g_{\text {pas }}=0.0002\left[\frac{\mathrm{S}}{\mathrm{cm}^{2}}\right]$.

For the axon,

$$
R_{m}=\frac{\tau}{C_{m}}=\frac{0.0003[\mathrm{~s}]}{0.000001\left[\frac{\mathrm{F}}{\mathrm{cm}}\right]}=300\left[\Omega \mathrm{cm}^{2}\right]
$$

Then $g_{\text {pas }}=0.00333\left[\frac{\mathrm{S}}{\mathrm{cm}^{2}}\right]$.

Axial specific resistivity can be obtained from the definition of the cable equation membrane resistance $r_{m}=\frac{R_{m}}{\pi d}$ and axial resistivity $r_{i}=\frac{4 R_{i}}{\pi d^{2}}$ (see Section 2.6.1), were $d$ is the cable diameter. The length constant is defined as $\lambda=\sqrt{\frac{r_{m}}{r_{i}}}$ then,

$$
\lambda=\sqrt{\frac{d R_{m}}{4 R_{i}}}
$$

and

$$
R_{i}=\frac{d R_{m}}{4 \lambda^{2}}
$$

For the dendrite,

$$
R_{i}=\frac{0.0005[\mathrm{~cm}] \times 5000\left[\Omega \mathrm{cm}^{2}\right]}{4 \times 0.00748225\left[\mathrm{~cm}^{2}\right]}=83.5310[\Omega \mathrm{cm}] .
$$


For the axon,

$$
R_{i}=\frac{0.0001[\mathrm{~cm}] \times 300\left[\Omega \mathrm{cm}^{2}\right]}{4 \times 0.00147456\left[\mathrm{~cm}^{2}\right]}=5.08626[\Omega \mathrm{cm}] .
$$

It might be of interest to have the membrane resistance $r_{m}=\frac{R_{m}}{\pi d}$ for the dendrite,

$$
r_{m}=\frac{5000\left[\Omega \mathrm{cm}^{2}\right]}{\pi \times 0.0005[\mathrm{~cm}]}=3.1831 \times 10^{6}[\Omega \mathrm{cm}]=3.1831 \times 10^{4}[\Omega \mathrm{m}] .
$$

For the axon,

$$
r_{m}=\frac{300\left[\Omega \mathrm{cm}^{2}\right]}{\pi \times 0.0001[\mathrm{~cm}]}=9.5493 \times 10^{5}[\Omega \mathrm{cm}]=9.5493 \times 10^{3}[\Omega \mathrm{m}] .
$$

The dendrite membrane capacitance $c_{m}=\pi d C_{m}$ is

$$
c_{m}=\pi \times 0.0005[\mathrm{~cm}] \times 1\left[\frac{\mu F}{\mathrm{~cm}^{2}}\right]=0.0015708\left[\frac{\mu F}{\mathrm{~cm}}\right]=1.5708 \times 10^{-7}\left[\frac{F}{\mathrm{~m}}\right] .
$$

The axon membrane capacitance $c_{m}=\pi d C_{m}$ is

$$
c_{m}=\pi \times 0.0001[\mathrm{~cm}] \times 1\left[\frac{\mu F}{\mathrm{~cm}^{2}}\right]=0.00031416\left[\frac{\mu F}{\mathrm{~cm}}\right]=3.1416 \times 10^{-8}\left[\frac{F}{\mathrm{~m}}\right] .
$$

\section{A.4 Example: calculating activating function injected current}

For a finite cable under a homogenous field the activating function corresponds to two current injections at the end of the cables (Section 2.7.4.1). For the cable with sealed end conditions [45] the injected current term corresponds not to the second, but the first derivative of the potential,

$$
\frac{1}{r_{i}} E_{s}=I_{s}
$$

An example value of this current injection is now shown and verified with the results of Rotem and Moses [73]. Numerically, the threshold values of the spatially constant electric field required to depolarize the membrane by $30 \mathrm{mV}$ are $458 \frac{\mathrm{V}}{\mathrm{m}}$ for a dendrite and $280 \frac{\mathrm{V}}{\mathrm{m}}$ for the axon [73]. The equivalent currents are for the dendrite,

$$
I_{s}=\frac{458\left[\frac{\mathrm{V}}{\mathrm{m}}\right]}{4.2542 \times 10^{10}\left[\frac{\Omega}{\mathrm{m}}\right]}=10.7658[\mathrm{nA}]
$$

where the cable axial resistance is found for the dendrite as,

$$
r_{i}=\frac{4 R_{i}}{\pi d^{2}}=\frac{4 \times 83.5310[\Omega \mathrm{cm}]}{\pi \times 2.5 \times 10^{-7}\left[\mathrm{~cm}^{2}\right]}=4.2542 \times 10^{8}\left[\frac{\Omega}{\mathrm{cm}}\right]=4.2542 \times 10^{10}\left[\frac{\Omega}{\mathrm{m}}\right]
$$

This implies that a current injection of $+10.77 \mathrm{nA}$ and a current injection of $-10.77 \mathrm{nA}$ at each end of a dendrite is equivalent to the dendrite being under a $458 \mathrm{~V} / \mathrm{m}$ field. 
For the axon this is

$$
I_{s}=\frac{280\left[\frac{V}{m}\right]}{6.47603 \times 10^{10}\left[\frac{\Omega}{m}\right]}=4.3236[n A]
$$

with

$$
r_{i}=\frac{4 R_{i}}{\pi d^{2}}=\frac{4 \times 5.08626[\Omega \mathrm{cm}]}{\pi \times 1 \times 10^{-8}\left[\mathrm{~cm}^{2}\right]}=6.47603 \times 10^{8}\left[\frac{\Omega}{\mathrm{cm}}\right]=6.47603 \times 10^{10}\left[\frac{\Omega}{\mathrm{m}}\right] .
$$

\section{A.5 Permittivity of the membrane}

In this section an estimate of the membrane permittivity $\varepsilon_{\Gamma}$ is found. The capacitance of a pair of parallel plates of area $A$ is given by the expression

$$
c_{m}=\frac{\varepsilon \varepsilon_{0} A}{d}
$$

where $\varepsilon$ is the material permittivity and $\varepsilon_{0}=8.854 \times 10^{-12} \frac{\mathrm{F}}{\mathrm{m}}=8.854 \times 10^{-10} \frac{\mathrm{F}}{\mathrm{cm}}=$ $8.854 \times 10^{-4} \frac{\mu F}{c m}$ is the permittivity of the empty space. Membrane thickness is estimated to be around $d=5 \mathrm{~nm}=5 \times 10^{-7} \mathrm{~cm}$ :

$$
\begin{gathered}
\frac{c_{m}}{A}=\frac{\varepsilon \varepsilon_{0}}{d} \\
1 \frac{\mu F}{\mathrm{~cm}^{2}}=\varepsilon \frac{8.854 \times 10^{-4} \frac{\mu F}{\mathrm{~cm}}}{5 \times 10^{-7} \mathrm{~cm}}=\varepsilon \times 1770.8 \frac{\mu F}{\mathrm{~cm}^{2}} \\
\varepsilon=5.6472 \times 10^{-4} \\
\varepsilon_{\Gamma}=\varepsilon \varepsilon_{0}=5 \times 10^{-7} \frac{\mu F}{\mathrm{~cm}}
\end{gathered}
$$

\section{A.6 Typical fields inside the membrane}

For a $5 \mathrm{~nm}$ thick membrane, a typical voltage difference of $50 \mathrm{mV}$ produces an incredibly large field,

$$
\frac{50 \times 10^{-3} \mathrm{~V}}{5 \times 10^{-9} \mathrm{~m}}=1 \times 10^{7} \frac{\mathrm{V}}{\mathrm{m}} .
$$

This value coincides with [155] p. 141. With current technologies a transcranial magnetic stimulation field induces maximum $1 \times 10^{3} \frac{\mathrm{V}}{\mathrm{m}}$. Inside the membrane's by-layer, the influence on the magnetically induced field is minimal compared to the internal field. 


\section{A.7 Typical current densities}

The following is a compilation of typical current values in electrophysiology simulations and experiments. These values were used to validate the results during this work and provide a better idea of what magnitudes are found in the real world. These are:

- In a single compartment simulation $(\mathrm{L}=20, \mathrm{R}=10)$ with the typical NEURON configuration, hh mechanism, and after a $0.5 \mathrm{nA}$ stimulation for $100 \mathrm{\mu s}$, an action potential produces currents with a peak of $+0.25 \mathrm{~mA} / \mathrm{cm} 2$ for the capacitive current, $-0.75 \mathrm{~mA} / \mathrm{cm} 2$ for the sodium current and $0.8 \mathrm{~mA} / \mathrm{cm} 2$ for the potassium current. Noting that $1 \mathrm{~mA} / \mathrm{cm} 2=0.01 \mathrm{nA} / \mu \mathrm{m} 2=1000 \mu \mathrm{A} / \mathrm{cm} 2$.

- [36] talks about peaks of 0.2 to $0.5 \mathrm{nA} / \mathrm{\mu m} 2$ equivalent to 20000 to $50000 \mu \mathrm{A} / \mathrm{cm} 2$ (going out of the soma and axon hilloc).

- On p.160 [155] shows membrane currents of about 200 to $700 \mu \mathrm{A} / \mathrm{cm} 2$ (0.002 to $0.007 \mathrm{nA} / \mathrm{cm} 2$ ) for a squid at $18 \mathrm{deg}$. (This does not imply this current leaves the cell)

- [155] also shows conductivities of $10 \mathrm{mS} / \mathrm{cm} 2$. At $10 \mathrm{mV}$ this gives current densities of $100 \mu \mathrm{A} / \mathrm{cm}$.

- The typical micro-pipette injection current is $1 \mathrm{nA}$. Assuming a tip area of 1 to 5 $\mu \mathrm{m} 2$ this gives a current density of 1 to $0.2 \mathrm{nA} / \mu \mathrm{m} 2=100000$ to $20000 \mu \mathrm{A} / \mathrm{cm} 2$.

- The Axon Guide [156] says regarding the patch clamp: "the currents measured are very small, on the order of picoamperes in single-channel recording and usually up to several nano-amperes in whole-cell recording".

- [33] reports a total membrane current output of $400 \mathrm{nA}$ peak for a $105 \mu \mathrm{m}$ radius spherical "source" during an action potential. That gives a surface density of $400 /$ $\left(4^{*} \mathrm{pi}^{*}\left(0.0105^{\wedge} 2\right)\right)=288716.45 \mathrm{nA} / \mathrm{cm} 2=288.72 \mu \mathrm{A} / \mathrm{cm} 2=0.002887 \mathrm{nA} / \mu \mathrm{m} 2$.

- [40] reports total membrane currents of $5 \mathrm{nA}$ at the $10 \mu \mathrm{m}$ soma during AP, for a spherical source this implies a current density of $5 /\left(4 *\right.$ pi $\left.*\left(0.001^{\wedge} 2\right)\right)=$ $397887.36 \mathrm{nA} / \mathrm{cm} 2=400 \mu \mathrm{A} / \mathrm{cm} 2=0.004 \mathrm{nA} / \mu \mathrm{m} 2$ (considering the soma a $20 \times 5$ $\mu \mathrm{m}$ cylinder the amount is $800 \mu \mathrm{A} / \mathrm{cm} 2)$.

- [9] reports extracellular electric fields of $2 \mathrm{mV} / \mathrm{mm}$, at a typical conductivity of 5 to $10 \mathrm{mS} / \mathrm{cm}$, this gives $20^{*} 5$ to $20^{*} 10 \mathrm{mV} / \mathrm{cm}^{*} \mathrm{mS} / \mathrm{cm}=100$ to $200 \mu \mathrm{A} / \mathrm{cm} 2=0.001$ to $0.002 \mathrm{nA} / \mathrm{\mu m} 2$. The $2 \mathrm{mV} / \mathrm{mm}$ value of the paper was measured experimentally.

- 3D simulations of a 20um diameter cell producing a Hodgkin-Huxley action potential produce current peak outputs of only $0.035 \mu \mathrm{A} / \mathrm{cm} 2$. Capacitor and ionic currents have peaks of 300 to $800 \mu \mathrm{A} / \mathrm{cm} 2$. 


\section{A.8 Electrophysiology Conventions}

The following is a compilation of electrophysiology conventions. As in the previous section this helped providing an initial validation of the results obtained numerically. They are:

- The sign conventions for current are: outward transmembrane current is positive; axial current flow into a compartment is positive; positive injected current drives the voltage in a positive direction [12].

- Transmembrane potential $V_{m}$ is the potential inside of the cell minus the potential outside.

- By convention, membrane current is defined as positive when positive ions leave the cell.

- By convention, membrane current is defined as negative when positive ions enter the cell.

- The resting potential of most cells is negative. If a positive current flows into the passive cell $V_{m}$ becomes less negative.

- Depolarization (as in an action potential with sodium ions) is caused by an inward positive current.

- The potential of the extracellular fluid is defined to be zero (far from the cell). When a neuron is inactive, the excess internal negative charge causes the potential inside the cell to be negative.

- The resting potential is an equilibrium point at which the flow of ions into the cell matches that going out of the cell (in the passive case that is the voltage dependent passive leak current versus a constant "dummy" current set to correspond to the resting potential).

- Positive inward electrode current (i.e. "injecting current") increases the positive charge inside and the membrane voltage becomes more positive. This is however a negative current (imagine a free flow of current through a sealed hole in the membrane). To keep the electrode current value positive $\left(I_{e}\right)$ it is given by convention the opposite sign of the ionic current in the equation: $I_{m}=I_{i o n}+I_{c}-I_{e}$ or $I_{c}=I_{m}-I_{i o n}+I_{e}$. In NEURON for example, setting a positive IClamp amplitude, depolarizes a passive cell. See also Dayan and Abbott [67] section 6.3: "The Cable Equation". 


\section{B CHASTE-Membrane Guide}

The following is the user's and programmer's guide to the tool presented in Chapter 6: CHASTE-Membrane. 


\section{ニニニニニニニニニニニニニニニニニニニニニニニニニニニニニニニニニニニニニニニニニニニニ}

$\mathrm{V} 1.131 .7 .2013$

V 1.014 .6 .2012

Welcome to ChasteMembrane. ChasteMembrane is a Finite Element simulation tool to model the electric properties of cell membranes in a conductive space. ChasteMembrane was developed for neuron modeling but, provided the right geometry and membrane conductances, other types of cells could be simulated. A description of the methods used in ChasteMembrane is available in [1].

ChasteMembrane is open source and is released under the same license terms as Chaste (GNU LGPL 2.1 or superior). Contributions from the user community are encouraged.

ChasteMembrane is based on Chaste (Cancer, Heart and Soft Tissue Environment), and a working, compiled version of it is required. ChasteMembrane uses the same $\mathrm{C}++$ coding structure, and building tools used in Chaste. Chaste is extensively documented and can be obtained at http://WwW.cs.ox.ac.uk/chaste

ChasteMembrane provides a C++ library to describe your own cell membrane simulations. Medium knowledge of $\mathrm{C}++$ with templates programming is required. Chaste development centers around a unit test approach. Every functionality implemented is complemented by automated test routines. Test routines are run periodically to alert the developer if something has broken in the code. It is recommended to follow this approach when using Chaste and ChasteMembrane. Test routines also provide an excellent guide to new developers, describing individual use of the different software components. Examples of the main functionalities of ChasteMembrane are exemplified as "tests". These tests are available in the projects/membrane/test folder and its usage will be described in this guide.

A basic standalone application of ChasteMembrane is also provided with this distribution. See "RUNNING SIMULATIONS WITH THE EXECUTABLE" for a description.

INSTALLATION

ニニニニニニニニニニニニ

Follow this items closely to install ChasteMembrane. Upon any problem, help for Chaste and ChasteMembrane can be obtained at the chaste mailing list: chasteusers@maillist.ox.ac.uk. ChasteMembrane is known to work in Linux Ubuntu 11.10 (x86_64, manually installed, not using the Ubuntu package manager), and openSuSE 11.4 (x86_64, manually installed). Other operating systems have not been tested, but it should in principle work for any other system where chaste can be compiled.

1) Obtain Chaste from http://www.cs.ox.ac.uk/chaste/download.html. ChasteMembrane depends exclusively on Chaste release 2.0 (On the downloads section chaste_release_2_0.tgz, file size 60.94MB). Trying an older or newer versions will fail.

2) Extract ChasteMembrane in Chaste's root directory. The projects/ folder should be merged with chaste's own. Inside the projects/membrane/ directory, this file, the default configuration files, mesh handling tools, the main source code and the stand alone ChasteMembrane application can be found.

3) Set up Chaste 2.0 but ** read first** the following sub-points specific to ChasteMembrane. There is an excellent guide for each Chaste version in the file 
docs/INSTALLATION.txt of Chaste's root folder. ChasteMembrane has not been tested with other compiler or system different from gcc or Linux. ChasteMembrane depends only on the "heart" and core packages so it is not required to build the entire set of Chaste libraries. The sub-points are:

- Before this make sure your Linux system has the gcc, g++ compiler, libncurses5-dev, and mesa development packages installed (libgl1-mesa-dev, libglu1-mesa-dev, libxext-dev, libx11-dev).

Without this packages you can get some weird building errors when trying to compile mpich, petsc, vtk or the ChasteMembrane examples.

- When setting up the file python/hostconfig/default.py (used to point to your own libraries e.g. petsc, boost), the VTK library has to be enabled and available for compilation. Set use_vtk = True. VTK is the default output format of ChasteMembrane. Compiling VTK requires the OpengL development libraries. For Linux the packages libgl1-mesa-dev, libglu-mesa-dev, libxt-dev and their dependencies have to be installed.

- VTK requires CMake to be installed. It is recommend not to compile cmake, but to use the CMake from the Linux distribution your are using.

Versions 2.6.2, 2.8.3 and 2.8.7 of cMake have been used successfully to generate the VTK make files.

- The recommended VTK version 5.2.1 has a bug in the VTK/Utilities/vtktiff folder. This folder should not have read only files, change this with the command: chmod u+w VTK/Utilities/vtktiff/*

- The boost program_options library is used by ChasteMembrane. Add this to the "other libraries" line in default.py:

other_libraries $=[$ 'boost_serialization',$\ldots$ ' 'metis',

$$
\text { 'boost_program_options'] }
$$

Make sure your boost installation contains the boost program_options library.

- Make sure you use the mpich version recommended for gcc $>4.3$ recommended in the installation instructions provided in Chaste. Also ignore the errors when installing mpich ("iostream.h no such file or directory")

- Instead of petsc-2.3.3 use petsc-3.๑.๑-p12. For an unknown reason tests run with petsc-2.3.3 failed (Ubuntu 11.10 x86_64 machine).

- Boost 1.34.1 produces compilation errors. Apparently version 1.34.1 is too old for recent versions of gcc. Boost 1.40.0 (boost_1_40_0.tar.gz) has been used without issues. The tool boost-jam-3.1.17-1-linuxx86.tgz can be used but in some 64 bit architectures it might need to be compiled again from the Sourceforge source.

- Ignore the hdf5 testing errors such as $" * * * * * 164$ FAILURES! $* * * * *$ Command exited with non-zero status $1 "$

- Many systems have a default installation of the MPI library. It is recommended to build and use the one suggested in the installation document. Other implementations of MPI had not been tested.

- Aside from the previous notes, use strictly the libraries suggested for Chaste release 2.0 in the installation document. As this is an old version of Chaste some of the source libraries described in the installation instructions are no longer available in the same URLs. Search for the same versions in Google.

- As with any other heavily dependent packages, building Chaste 
might be a complex process. Ask the mailing list upon any doubt!

4) Follow chaste guides to compile and run the monodomain problem test. Make first sure the right environment variables have been set. Chaste installation recommends running the automated tests for the entire heart package. This is preferred as it can tell everything was set up properly. Still, a single test such as heart/test/monodomain/TestMonodomainProblem.hpp would be enough to check all dependencies of ChasteMembrane are met. This is a quick way to jump to ChasteMembrane own tests. Before compiling the monodomain example, the following changes in the Python scripts are needed:

- In Linux install the following packages required for compilation of Chaste examples: zlib1g-dev

- Remove the file python/hostconfig/ubuntu.py from the source code folder. This file is broken and it is not required for this type of installation.

- Edit the file python/hostconfig/default.py and comment the line \#chaste_libs_path $=1 /$ home $/$ scratch/chaste-libs/' and add the line: chaste_libs_path $=$ os.environ['CHASTE_LIBS']+'/'

- Remove the petsc_2_2_path and petsc_2_3_path variables also in default.py by commenting the lines: \#petsc_2_2_path = '' \#petsc_2_3_path = chaste_libs_path+'petsc-2.3.3-p15/' \#os.path.join(petsc_2_3_path, 'externalpackages/f2cblaslapack/linux-gnu'), and adding the lines:

petsc_3_0_path = chaste_libs_path+'petsc-3.0.0-p12/'

- In defaults.py change the other_includepaths variable commenting out \#chaste_libs_path+'boost/include/boost-1_34_1', and adding chaste_libs_path+'boost/include',

- If you haven't done so, add the boost program options library required by ChasteMembrane:

other_libraries $=[$ 'boost_serialization', 'xerces-c',$\ldots$, and set: 'boost_program_options']

use_vtk $=$ True

- In gcc versions 4.6 or superior there might be issues with the default chaste build flags. The following change should be made to python \BuildTypes.py in line 51: self._cc_flags $=[$ '-Wall', '-Wno-error=unused-but-set-variable', to avoid compilation errors. '-fpermissive', '-include unistd.h']

Compiling and running the test requires the command:

scons test_suite=heart/test/monodomain/TestMonodomainProblem.hpp

Compilation might take some minutes. At the end of the test, the final message will say that 1 of the 17 tests has failed

(TestMonodomainProblemCreates3DGeometry), ignore this. Also ignore any HDF5 error messages.

5) After running the TestMonodomainProblem test, everything should be ready to run ChasteMembrane tests. Tests should always be run from the chaste root

folder. Start by running: 
scons test_suite=projects/membrane/test/TestCell2D.hpp

And then:

scons test_suite=projects/membrane/test/TestCell3D.hpp

A message of "Passed" should appear after each test. To run all basic tests use: scons projects/membrane

OUTPUT

$=====$

ChasteMembrane produces its output as a set of data files. This files are located in Chaste's default output folders. By default this is the system's /tmp/username folder. Read Chaste's installation guide to change the default location.

The following output files are produced by ChasteMembrane in the test output root folder:

defaults.txt: a copy of the default configuration used in the current version.

*. conf: a copy of the configuration used and the command line parameters for a specific simulation (see also the "CONFIGURATION" section).

The following files are produced by ChasteMembrane in the test output folder:

*.dat: files containing the PETSc binary matrices and vectors used by the linear solver. (This files are for debugging purposes and will not be produced in future versions.)

*.h5: An HDF5 file with the potentials at each node and each time-step. It follows Chaste's default monodomain potential output format.

*_membrane.h5: An HDF5 file with the voltages on the membrane at each node and each time-step. It also follows chaste's default monodomain potential output format. The values correspond to membrane nodes only.

currents.txt: A text file with the voltage and currents for a specific point of the membrane (see also the "CONFIGURATION" section).

The following files are produced in the folder "vtk_output" inside the test output folder:

cha.vtu: A VTK visualization file with $\mathrm{n}$ variables, where each variable is the potential at each "printing" time step. This file can be converted to sequential vtu files with the tool:

./projects/membrane/tools/cha2vtu.py

This script requires the python-vtk package.

call this script without parameters for help information.

The rest of files in the output are produced by Chaste. A membrane *.vtu file is not yet produced but will be in future versions.

RUNNING OTHER TEST PROGRAMS

The test programs provided with the tool exemplify basic functionalities. These are located in the projects/membrane/tests folder: 
TestCell2D.hpp: A simple test with a diameter 20 um circular cell in a $400 \times 400$ um domain. The cell is stimulated with a homogeneous electric field. A similar example was presented in the convergence tests of [1]. Run as:

scons test_suite=projects/membrane/test/TestCell2D.hpp

TestCell3D.hpp: A simple test with a diameter 15 um spherical cell in an $80 \times 80$ um domain. The cell is stimulated with a homogeneous electric field. This corresponds to the spherical body presented in the results section of [1]. Run as:

scons test_suite=projects/membrane/test/TestCell3D.hpp

TestCable3D.hpp: An 80 um long, 1um diameter cable in a 100x10x10um domain. The cable is stimulated with a homogeneous field. This corresponds to the short cable presented in the convergence section of [1]. Run as:

scons test_suite=projects/membrane/test/TestCable3D.hpp

TestTimeDependentBC.hpp: Example of time dependent boundary conditions. In this version time dependent conditions can only be set through $\mathrm{C}++$ code. It uses the same mesh used in TestCell3D.hpp. Run as: scons test_suite=projects/membrane/test/TestTimeDependentBC.hpp

TestMultistimuli.hpp: Shows how to set up more than two point source stimuli. Point source stimuli require calculating the node's surrounding volume and this is also shown. Run as: scons test_suite=projects/membrane/test/TestMultistimuli.hpp

TestMembraneCurrent3D.hpp: Simple example of how to inject current inside a cell and obtain the membrane current values. Run as: scons test_suite=projects/membrane/test/TestMembraneCurrent3D.hpp

TestTwoCells3D.hpp: Reproduces the simulation of two nearby 3D cell bodies presented in [1]. It shows the use of heterogeneous membrane properties (different for each cell) and time dependent current point sources. Run as: scons test_suite=projects/membrane/test/TestTwoCells3D.hpp

TestNeuronOnDish2D.hpp: Corresponds to the realistic cell simulation of [1] . It exemplifies the use of ionic kinetics, heterogeneous membrane properties along a cell, and heterogeneous boundary conditions over mesh boundary regions. Run as: scons test_suite=projects/membrane/test/TestNeurononDish2D.hpp

The configuration files in projects/membrane/tests/data for each of the test show the parameters used in each simulation. See the CONFIGURATION section for a description of the parameters.

CONFIGURATION

Apart from the default configuration method (Chaste's XML file), ChasteMembrane 1.0 brings a separate configuration system. ChasteMembrane can be configured with command line parameters and/or a INI like *.conf file. The simpler PARAMETER=VALUE format was preferred by using the Boost::program_options library. Note this configuration method was found to be limited and other formats could be used in future releases (namely JSON or XML itself).

Some configuration parameters are specified in both ChasteMembrane and Chaste. Configurations in the *. conf file have priority over Chastes's *.xml file. A specific Chaste *.xml file can be specified with the CHASTE_CONFIG variable in 
the *.conf file. By the default, the file:

projects/membrane/ChasteMembrabeDefault.xml is used.

Configuration through command line can be provided with the syntax

- -PARAMETER=VALUE or --PARAMETER VALUE. Any parameter set in the command line has priority over the *.conf file. If the same parameter is specified in both the command line and the *.conf file the latter will be ignored. Ideally, the only configuration parameter passed through command line should be:

- -CONFIG=file.conf. This file can provide all remaining settings (e.g. the mesh file to be used). This is however not restrictive and other values can be passed to quickly test different experimental settings (Example: --CONFIG=file.conf - - DURAT=1.0).

Default parameter values are specified in the MembraneConfig class. If a *.conf file is not provided, ChasteMembrane will use the file:

projects/membrane/ChasteMembrabeDefault.conf

This runs a basic 2D simulation similar to TestCell2D.hpp. A user configuration file can either be provided in the command line or through $\mathrm{C}++$ code with the call:

MembraneConfig: : Instance( )->SetParametersFile("...");

Note the object MembraneConfig: Instance() should not be used to manually set parameters.

See the test example codes presented in "RUNNING OTHER TEST PROGRAMS" for usage of the MembraneConfig class.

The list of default configuration values and a description for each parameter is provided in the file projects/membrane/default.txt.

Setting the following environment variables is recommended:

export CHASTE_LIBS=/home/user/chaste

export CHASTE_SRC=/home/user/chaste

export PATH=\$CHASTE_LIBS/bin: \$CHASTE_SRC/projects/membrane/tools: \$PATH

export LD_LIBRARY_PATH=\$CHASTE_LIBS/petsc-3.๑.๑-p12/lib/linux-gnu: \$CHASTE_LIBS/rdf/lib:\$CHASTE_LIBS/boost/lib: \$CHASTE_LIBS/xerces/lib:\$CHASTE_SRC/lib

export PYTHONPATH=\$CHASTE_LIBS/python

export CHASTE_TEST_OUTPUT=/home/user/chaste-out

Change /home/user/chaste for the directory were you built chaste and ChasteMembrane.

BUILING YOUR OWN TEST PROGRAMS

ChasteMembrane can be used as a stand alone executable or as a c++ library. The executable can be used to quickly run simulations parametrized in the command line or a configuration file. To access more powerful features, C++ must be used. This extended features include: setting heterogeneous channels, writing custom channel kinetics, and using heterogeneous and time dependent boundary conditions. Use of heterogeneities is exemplified in the test examples. Example channel implementations are available in the projects/membrane/src/odes/ionicmodels directory and in chaste's heart/src/odes/ionicmodels.

To create your own simulation it is recommended to use one of the example test programs as a template. ChasteMembrane uses the coding structure of Chaste. All the documentation and functionalities of Chaste are available to the programmer of new ChasteMembrane simulations. 
To build them, test programs should be located in the projects/membrane/test/ directory. The name of your test functions should always start with Test*. See Chaste and cxxtest documentation for more information. To build your own test use :

scons compile_only=1 test_suite=projects/membrane/test/TestYourClass.hpp

To build and run the test use:

scons test_suite=projects/membrane/test/TestYourClass.hpp

In this form, alternative command line parameters cannot be added. Command line parameters can be passed by executing the test binary directly:

./projects/membrane/build/debug/TestYourClassRunner [Command line parameters]

MESH GENERATION

ニニニニニニニニニニニニニニニ

ChasteNeuron uses tetgen/triangle mesh format exclusively. Besides the regular tetgen/triangle mesh files, special files should be provided to describe the interfaces (membranes) in the mesh. A Python tool to produce this files is provided in: projects/membrane/tools/msh2tetgen.py. This tool takes as input a Gmsh *.msh file and produces the corresponding tetgen/triangle files. Gmsh is a widely used open source and free CAD/meshing engine that can be obtained at http://geuz.org/gmsh/

For information about usage of msh2tetgen.py call:

./projects/membrane/tools/msh2tetgen.py -h

For a description of the format of the Gmsh mesh see:

projects/membrane/tools/gmshint.py

Examples of Gmsh *.geo files and their corresponding *.msh and tetgen/triangle files are available in projects/membrane/test/data

RUNNING SIMULATIONS WITH THE EXECUTABLE

The executable (.projects/membrane/apps/src/ChasteMembrane) can run ChasteMembrane simulations if they are described in a configuration file or through command line. For example:

./projects/membrane/apps/src/ChasteMembrane - -CONFIG=file.conf

With configuration files, any mesh can be simulated with Dirichlet boundary conditions and one or two point source currents. Any property available through the configuration interface can be used. See "CONFIGURATION" for all the possible parameters.

To use it, the executable has to be built first. First build Chaste main binary shared libraries with:

scons chaste_libs $=1$ exe $=1$ compile_only=1 apps

These libraries have to be added to the LD_LIBRARY_PATH environment variable of chaste as (in a single line):

export LD_LIBRARY_PATH=\$CHASTE_LIBS/petsc-3.0.0-p12/lib/linux-gnu:

\$CHASTE_LIBS/rdf/lib:\$CHASTE_LIBS/boost/lib:\$CHASTE_LIBS/xerces/lib:

\$CHASTE_SRC/lib 
This will build Chaste's shared libraries. A helper script is provided to build the ChasteMembrane executable. From the chaste root folder call:

./projects/membrane/apps/src/build_chaste_membrane.sh

This script depends on scons and the same Chaste environment variables used for building tests. After completion, the executable can be used as specified above.

NOTES FOR VERSION 1.0

Version 1.0 could be considered a prototype and many features that would be considered "User friendly" have not been added. Still, all functionalities can be accessed by using ChasteMembrane as a C++ library. Future releases will improve the use of ChasteMembrane as a standalone application. Suggestions and patches by the community are also welcomed. Important notes are:

- Using configuration files only two source currents can be set. More currents can be added with C++ code. See projects/membrane/test/TestMultistimuli2D.hpp for an example. Stimulus currents should be given to the solver as volume sources $(\mathrm{uA} / \mathrm{cm} 3)$. This requires calculating the area or volume surrounding the point source. This is described in the example.

- For the source current 1 (SRC1_FILE) the time values of the *.csv file are ignored. Also, the number of values should match the simulation steps.

- Only an initial membrane voltage for all cell is supported via configuration parameters. This can be changed through $\mathrm{C}++$ routines. Gmesh mesh regions can be used to specify this values.

- In this version, simulations can be run in parallel as long as all the membrane nodes are assigned to an individual processing core. For this, all the nodes touching the membrane should be sequential and at the end of the node list (this is the default ordering produced by msh2tetgen.py). With Chaste's default partition scheme, this means that the number of nodes touching the membrane should be less than $\mathrm{N} / \mathrm{n}, \mathrm{N}$ the number of nodes, and $\mathrm{n}$ the number of processors. Support for partitioned membranes will be added in the next immediate release of ChasteMembrane.

KNOWN ISSUES

ニニニニニニニニニニニニ

- CGG 4.6 brings the compiler option "-Wno-error=unused-but-set-variable" and this is enabled by the options -wall -Werror (at least in Ubuntu 11.10, GCC 4.6.1). You might need to modify the file python/BuildTypes.py disabling this flag:

self._cc_flags $=[$ '-Wall', '-Werror', '-Wno-error=unused-but-set-variable']

- The file *.int.msh produced by msh2tetgen.py displays the two edges (inner interfaces) corresponding to the physical groups 4 and 5 . This is because gmsh consider elemental geometry IDs are always associated to a single physical group. However, as the internal interface is duplicated in the conversion process they share the same element id's. This is just a visualization problem and does not affect the resulting meshes.

\section{REFERENCES}

[1] Andres Agudelo-Toro, Andreas Neef. Computationally efficient simulation of electrical activity at cell membranes interacting with self-generated and externally imposed electric fields. 2013 J. Neural Eng. 10026019. 


\section{Applications of the space/membrane solution: transcranial magnetic stim- ulation}

\section{C.1 Introduction}

The human brain lives inside a protective shell that screens it from outside intervention. Non-invasive neurological stimulation treatments are ideal as they relieve from unnecessary trauma and pain. Transcranial Magnetic Stimulation (TMS) overcomes this by inducing an electric fields in a small area of the cortex using focused magnetic pulses. The magnetic pulses are delivered with a current coil over the skull and not touching the patient's head. TMS has great potential for the treatment of neuro-psychiatric disorders [157] for example as a replacement for electroconvulsive therapy [13, 158]. As a research tool, TMS has been used in neuroscience research to induce localized "virtual lesions" [159, 160, 161] allowing to probe brain specific regions by disruption of function.

The technology for transcranial magnetic stimulation has basically remained unchanged from its origins in the late 80's [14]. Previous developments have aimed at a higher pulse repetition rate [162] and at a larger penetration range [163]. However a main characteristic of TMS is the strong directionality of the applied field [164, 165, 166, 167, 168, 169]. Optimal stimulation is achieved when the external field is aligned with the cell processes. Neurons in the targeted brain area seem to be excited only if the induced electric field is aligned with their axons [73]. Determining which orientation of the coil is optimal is then crucial for effective stimulation. Still, the optimal coil orientation may not be found if stimulation of the targeted brain region does not lead to a direct reaction (e.g. muscle movement). This is for instance the case for the dorsolateral prefrontal areas that are targeted in depression. Therefore a device that ameliorates the directional sensitivity and enables a more efficient mode of applying TMS is a clear goal for development of coils.

The "dense cloverleaf" coil configuration, devised by Rotem et al. [170] is similar to currently available coils but produces a rotating electric field. A rotating field can mitigate the directional sensitivity by scanning all the possible processes angles in a short time. The cloverleaf coil consists of two "figure of eight" coils shaped near the hotspot to maximize the field strength. Each figure of eight is connected to an independent power source. The two figure of eight coils are positioned on the same plane and perpendicular to each other so that at the hotspot, their resulting electric fields 


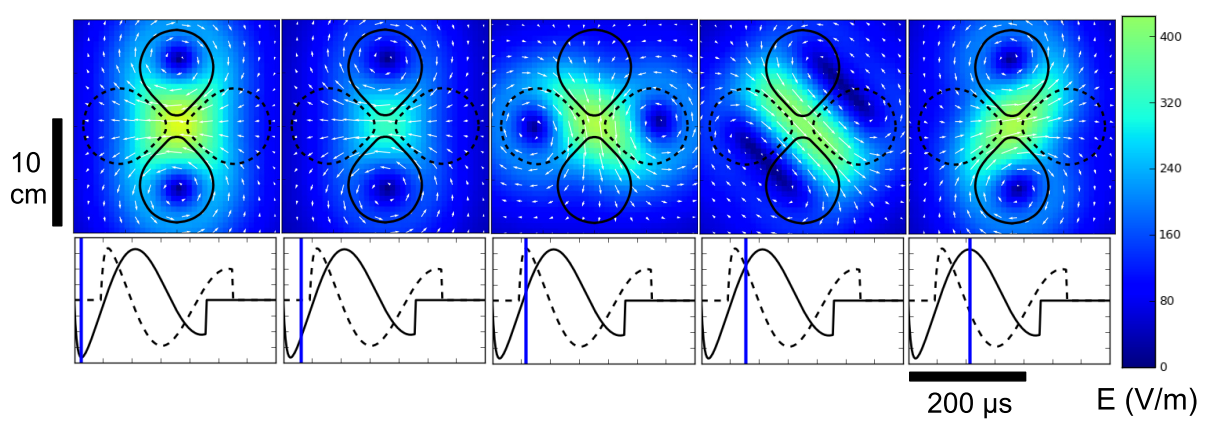

Figure A.1: Rotating cloverleaf coil in action. The induced electric field is displayed on top while the magnitude over time is displayed in the bottom.

\begin{tabular}{|c|c|}
\hline$\left(d l, \frac{d l}{2}[\mathrm{~mm}]\right)$ & $d_{j}$ \\
\hline \hline$(10,5)$ & 0.9681 \\
\hline$(5,2.5)$ & 0.2207 \\
\hline$(2.5,1.25)$ & 0.1340 \\
\hline$(1.25,0.625)$ & 0.0861 \\
\hline$(0.625,0.3125)$ & 0.0170 \\
\hline$(0.3125,0.015625)$ & 0.0154 \\
\hline
\end{tabular}

Table A.1: Convergence of the field magnitude.

are perpendicular. The quasi-sinusoidal pulses of the pairs are separated by a phase of 90 degrees. The resulting electric field at the hotspot completes $\frac{3}{4}$ of a circle during one combined pulse (Figure A.1). The rest of the characteristics of the electric field, in terms of strength, focus and decay on the Z-axis are similar to that of the conventional TMS coils.

This chapter presents numerical simulations that helped validate the design and effectiveness of the dense cloverleaf coil. In collaboration with A. Rotem and E. Moses I developed models of the coil and the induced field and tested them in artificial axons. The cable version of the space/membrane equations combined with the extension of the quasi-static approximation to allow magnetic induction (Eq. (2.57)) was used for this simulations. A custom made coil simulator had to be build to calculate the induced electric field $\frac{\partial \mathbf{A}}{\partial t}$. In the next sections this coil model tool and the results obtained for validation this specific coil design are presented. The effects of the coil stimulating a model axon are then shown.

\section{C.2 The dense cloverleaf coil model}

\section{C.2.1 Numerical tool}

The electric field induced by the dense cloverleaf coil was numerically estimated with a custom written $\mathrm{C}$ program. The numerical model was constructed to reproduce the exact geometry of the physical coil and in general any coil shape. The model calculated 

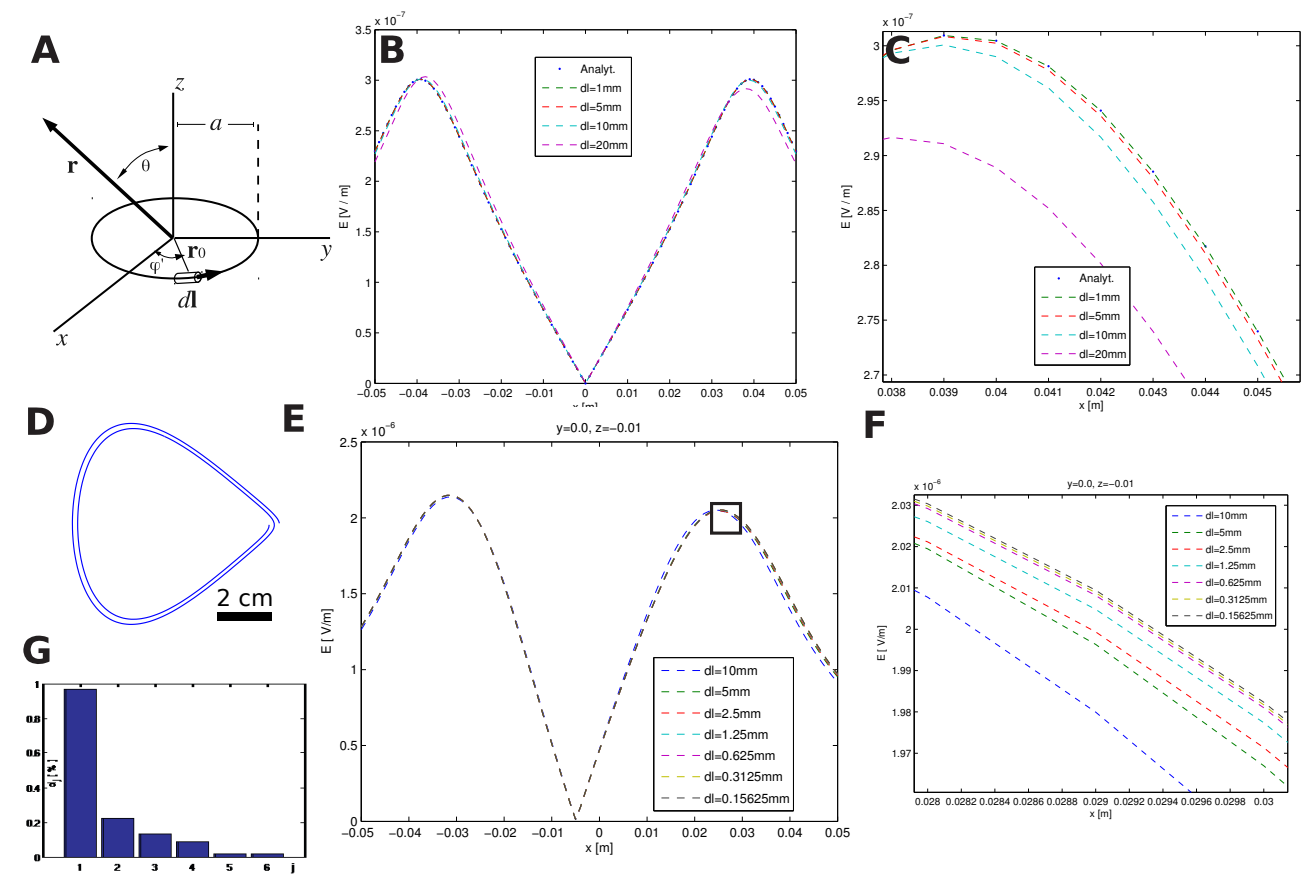

$\mathbf{F}$

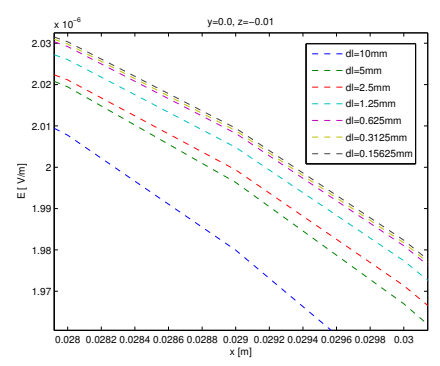

Figure A.2: Difference between different solutions with increasingly smaller length differential.

the stimulus electric vector field (Eq. (2.57))

$$
\mathbf{E}_{s}(\mathbf{r}, t)=-\frac{\partial \mathbf{A}(\mathbf{r}, t)}{\partial t}
$$

for a given three-dimensional coordinate $\mathbf{r}$. The vector potential field $\mathbf{A}$ can be written for a spatially homogeneous coil current $I(t)$ in a wire piece $d \mathbf{l}$ as the path integral

$$
\mathbf{A}(\mathbf{r}, t)=\frac{\mu_{0}}{4 \pi} I(t) \int_{C} \frac{d \mathbf{l}}{\left|\mathbf{r}-\mathbf{r}_{0}\right|}
$$

with $\mathbf{r}_{0}$ a vector pointing to the differential unit of the cable and $\mathbf{r}$ the vector at the point of interest (see also Figure A.2(A)). The electric field can then be described by the expression

$$
\mathbf{E}_{s}(\mathbf{r}, t)=-\frac{\partial \mathbf{A}(\mathbf{r}, t)}{\partial t}=-\frac{\mu_{0} N}{4 \pi} \frac{\partial I(t)}{\partial t} \int_{C} \frac{d \mathbf{l}}{\left|\mathbf{r}-\mathbf{r}_{0}\right|} .
$$

Equation (C.2) was calculated numerically for arbitrary wires. To validate the numerical results, an analytical solution of (C.1) was compared to the numerical for an identical geometry. For a single loop of current centered at the origin, the field produced is cylindrically symmetric and the vector potential can be calculated for a single Cartesian plane. In spherical coordinates, a vector pointing out of the XZ plane has its single component described by the $\phi$ coordinate. The expression for $\mathbf{A}$ is [72]

$$
A_{\phi}(r, \theta)=\frac{\mu_{0} I a}{4 \pi} \int_{0}^{2 \pi} \frac{\cos \phi^{\prime}}{\left(a^{2}+r^{2}-2 a r \sin \theta \cos \phi^{\prime}\right)^{1 / 2}} d \phi^{\prime}
$$



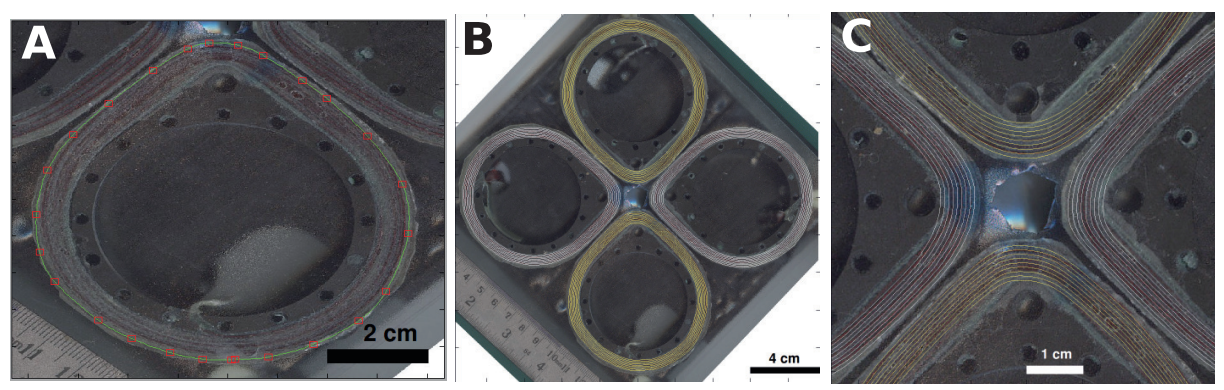

Figure A.3: Spline reconstruction of the coil. (A) Spline (green) identifies the borders of the coil. (B-C) the final reconstructed spline of the coil.

With $a$ the loop radius and the rest of the notation as in Figure A.2(A). This in turn can be solved in terms of the complete elliptic integrals $K(m)$ and $E(m)$ [171]

$$
A_{\phi}(r, \theta)=\frac{\mu_{0}}{4 \pi} \frac{4 I a}{\left(a^{2}+r^{2}-2 a r \sin \theta\right)^{1 / 2}}\left(\frac{(2-m) K(m)-2 E(m)}{m}\right)
$$

with $m$

$$
m=\frac{4 a r \sin \theta}{a^{2}+r^{2}-2 a r \sin \theta}
$$

Comparison to the numerical results is presented in Figure A.2(B-C). The numerical solution converges for the loop of current.

Additional tests were performed for a coil with the clover leaf shape of Figure A.2(D). With reduced elements it was verified that the solutions did not diverge. The difference between solutions was calculated with

$$
d_{j}=\frac{100 \%}{\max E_{i}^{d l \rightarrow 0}} \sum_{i=1}^{N} \frac{\left|E_{i}^{d \mathbf{l}}-E_{i}^{\frac{d \mathbf{l}}{2}}\right|}{N}
$$

for $E_{i}$ the field magnitude calculated at points $i$ separated $1 \mathrm{~mm}$ along the $x$ axis 1 $\mathrm{cm}$ far from the coil. The length differential $d \mathbf{l}$ was halved for every comparison. The results are shown in Figure A.2(E-G) and Table A.1.

\section{C.2.2 Coil reconstruction}

To represent the geometry in the computer, a high resolution image of the coil was obtained with a regular office scanner. The image was used to manually match a 3rd order polynomial Catmull-Rom spline [172] describing the exterior wire loop in the XY plane (Figure A.3(A)). The exterior loop of each leaf was then copied and rescaled to match 9 wire loops forming a complete coil (Figure A.3(B-C)). The complete set of loops was then copied five times, creating 6 layers in Z, representing the coil height. For numerical integration each spline describing a complete wire loop was subdivided in $1 \mathrm{~mm}$ linear sections. 

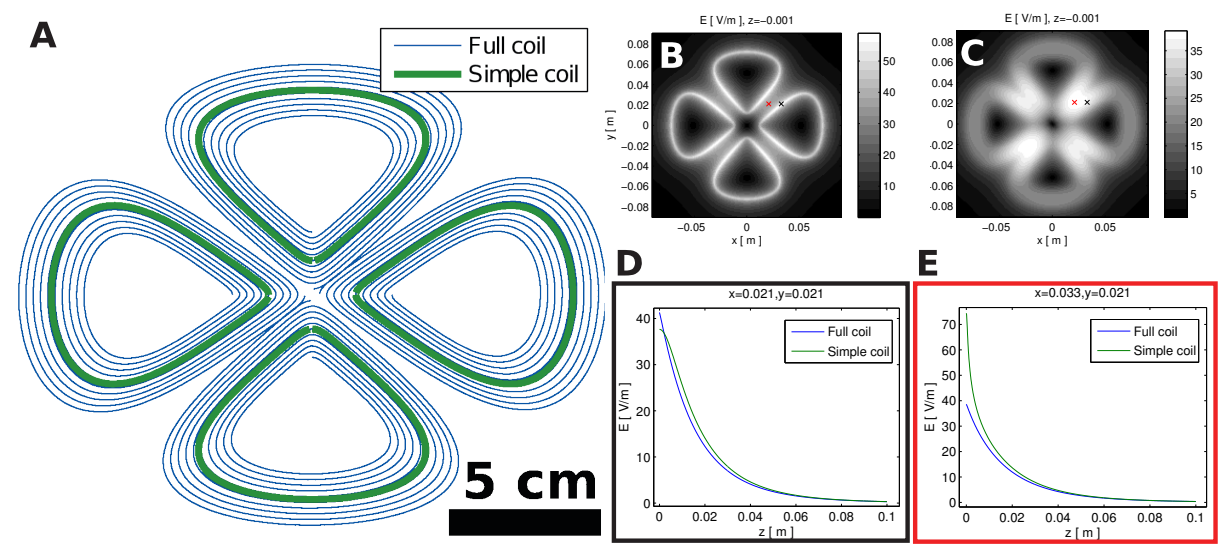

Figure A.4: (A) complex vs. simple coil. (B-C) The field produced by the coils in the plane. (D-E) The field produced by the coils in $\mathrm{Z}$.

The inclusion of each of the cable lops in the simulation, and not just representing the coil as a single wire, improves the accuracy of the field estimate. This is particularly true within the first 2-4 centimeters below the coil ([173] and Figure A.4). The design of the dense cloverleaf is optimal compared to circular and wedged coils. The smooth central region maximizes the induced field strength as shown in Figure A.5. The current is assumed homogeneous along the cables so expression (C.2) can be separated in a time dependent and a time independent part. For the dense cloverleaf, as each pair of leaves is feed by an independent current source the field was only calculated once for each pair and scaled over time. The rotating field simulation was presented in Figure A.1.

\section{C.3 Simulation of axonal excitation}

\section{C.3.1 Passive axon}

In order to check the efficacy of the dense cloverleaf the electric field induced was used to drive an artificial axon. At the coil hotspot, the magnitude of the rotating field varies over time and over its angle. The projection of the field along an axon at any given orientation should be large and long enough to produce depolarization. For this the coil model tool and the one-dimensional cable solver tool presented in appendix Chapter E were used. The axon was simulated as single passive unmyelinated cable of length $1 \mathrm{~mm}$ and diameter $1 \mu \mathrm{m}$. The length constant of the cable was $\lambda=384 \mu \mathrm{m}$ and the time constant was $\tau=300 \mu$ s, as justified by a previous study [73]. It was assumed the axon lied in the hotspot of the coil around $3 \mathrm{~cm}$ below the coil center. The axon was short enough so that the spatial variation of the electric field could be ignored. The axon was divided in 101 segments and a fixed time step of 2 ps was used during the simulations. It was determined that a $30 \mathrm{mV}$ depolarization was needed to produce stimulation.

The results of this simulations are presented in Figure A.6. The axon was set along the $\mathrm{X}$ axis so that only the projection $E_{x}(t)$ of the electric field was effective. The amplitude of the input current to the coil was then tuned in a binary search procedure 

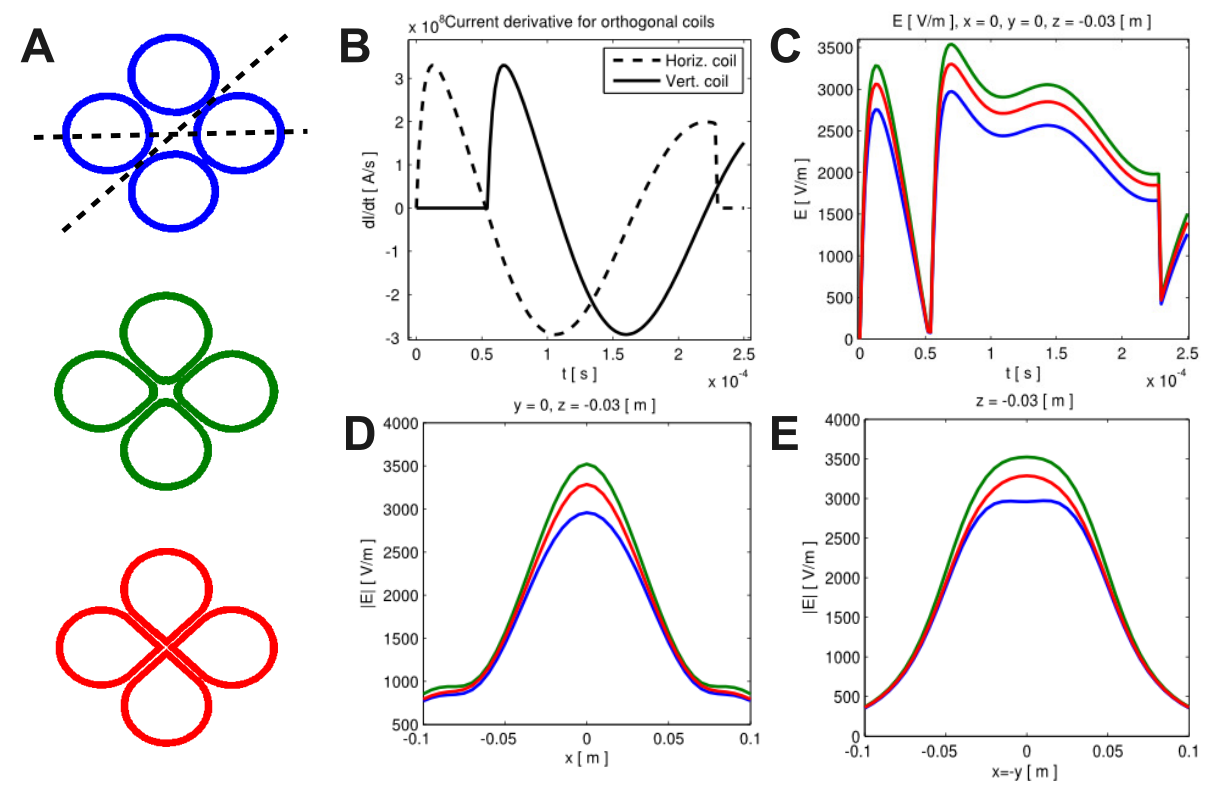

Figure A.5: Comparison of several coil shapes (A). (B) The horizontal and vertical components of the currents. (C) Time evolution of the magnitude of the induced field. (D-E) Field magnitudes at peak stimulus for the dashed lines in (A).

to find the electric field magnitude at which the axon was depolarized by $30 \mathrm{mV}$ at any point along it. This electric field value was then chosen as the electric field threshold and plotted. The coil was then rotated in 30 degrees intervals and the same procedure repeated. The plot in A.6 shows the normalized effective induced current (proportional to the field) and the membrane voltage response at the right tip of the axon for each angle. Note that at zero degrees the coil had the same orientation that in Fig. A.1 but the current of the vertical coil (solid trace) was inverted. The coil is effective for all angles presenting threshold fields that change by less than $50 \%$.

\section{C.3.2 Active axon}

An identical test was performed but active properties were given to the axon. The voltage dependent sodium conductances were taken from the first model of Baranauskas and Martina [174] and the potassium and leak conductances of Migliore et al. [175]. The total sodium conductance was increased from $65 \mathrm{mS} / \mathrm{cm} 2$ in Baranauskas and Martina to $120 \mathrm{mS} / \mathrm{cm} 2$. The threshold surpassing criteria was then generation of an action potential. The thresholds for this case are presented in Figure A.7. Although the threshold increases compared to the passive cable, the field strength remains within 300 to $400 \mathrm{~V} / \mathrm{m}$. 


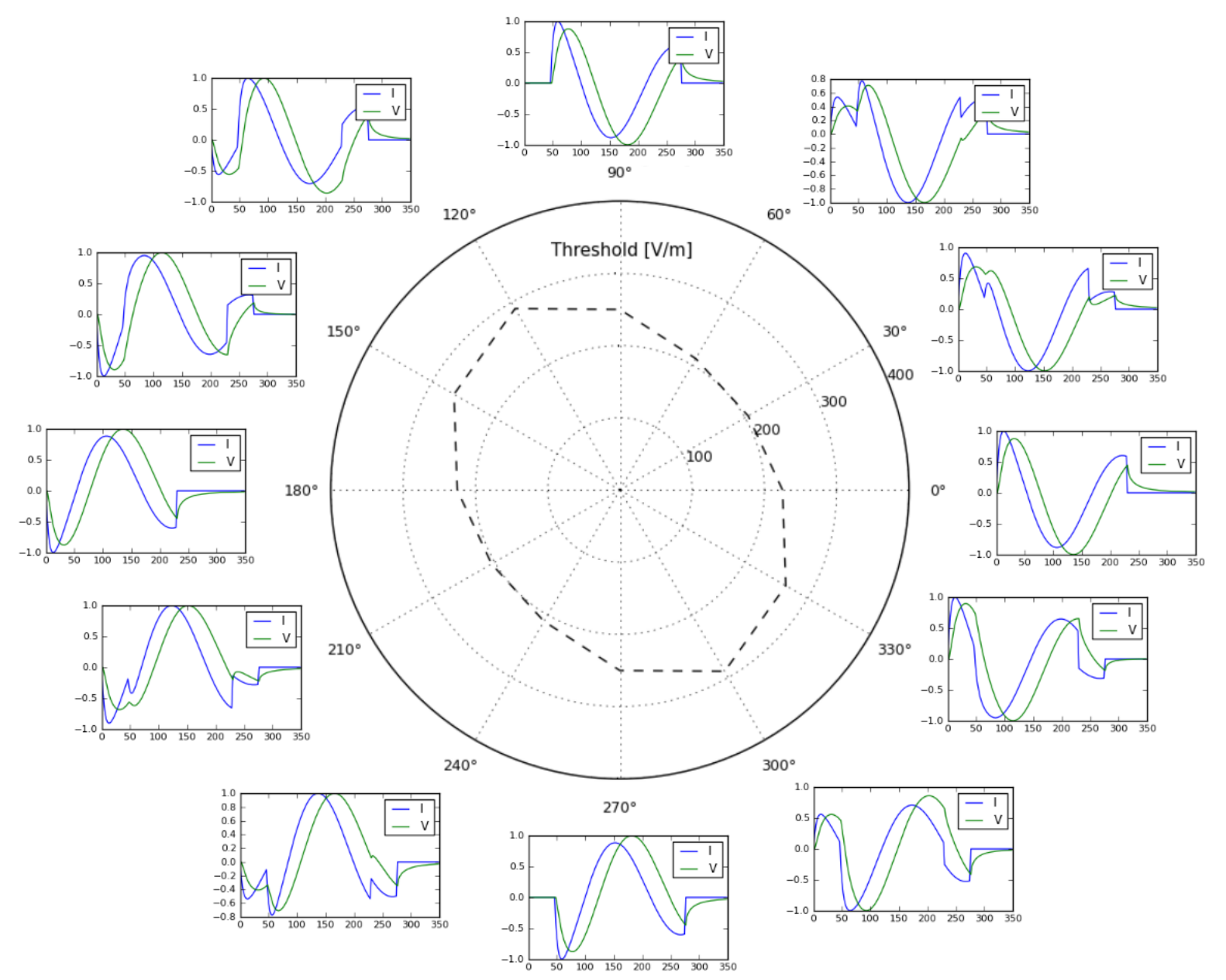

Figure A.6: The cloverleaf coil alleviates the orientation dependence of magnetic stimulation in a passive axon. 


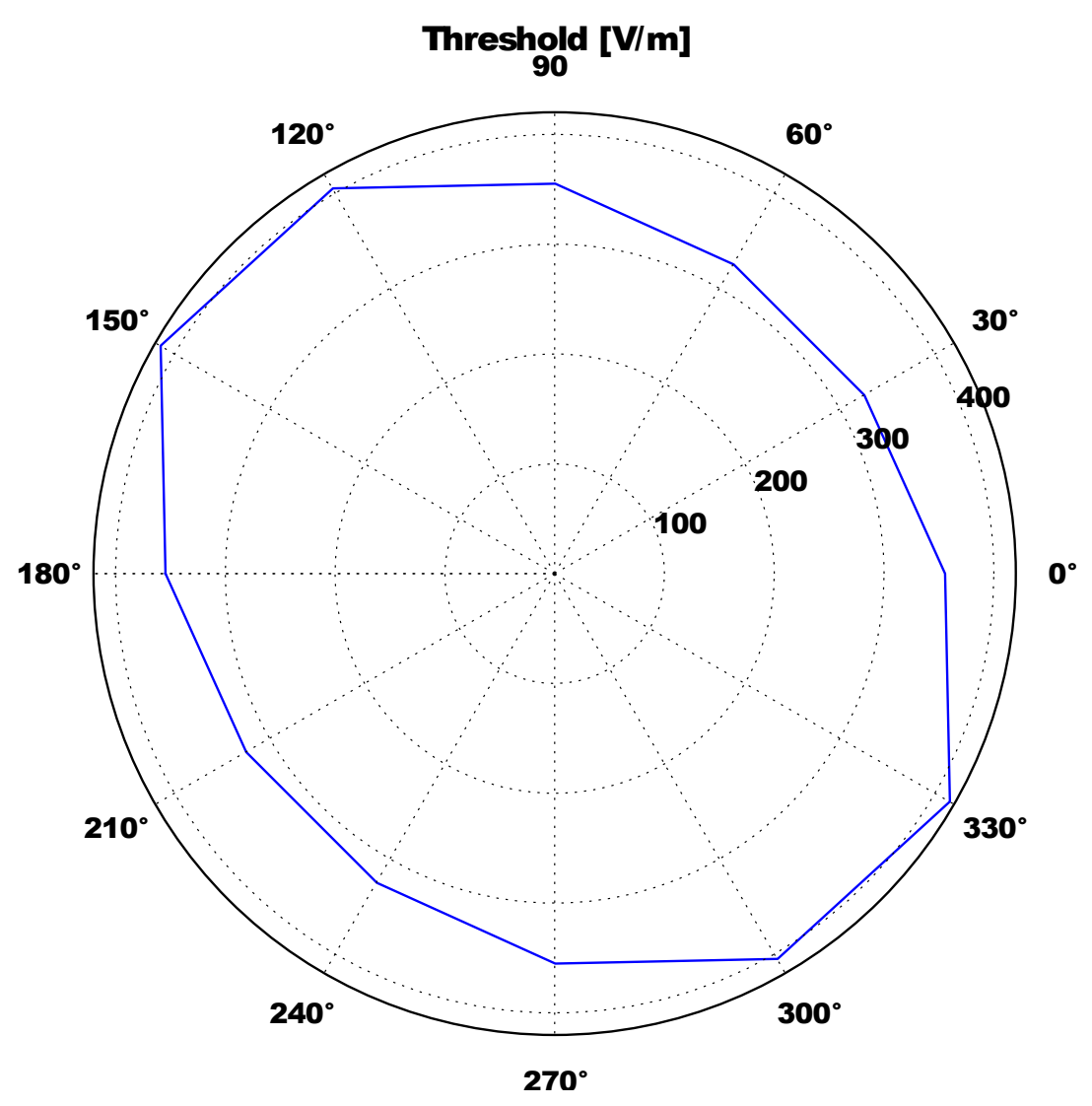

Figure A.7: The cloverleaf coil alleviates the orientation dependence of magnetic stimulation in an active axon. 


\section{UML Design diagrams}

The following figures present the UML (Unified Modeling Language, [128]) diagrams created during the development of CHASTE-Membrane. The use of UML allowed to visualize the relation between components and correctly interface the new functionalities with CHASTE. Diagrams A.1 and A.2 represent the object oriented inheritance relations of the objects constructed in CHASTE-Membrane in relation with the core objects of CHASTE. The solving sequence is presented in diagrams A.3 to A.5. These diagrams are key to understand the $\mathrm{C}++$ implementation. 


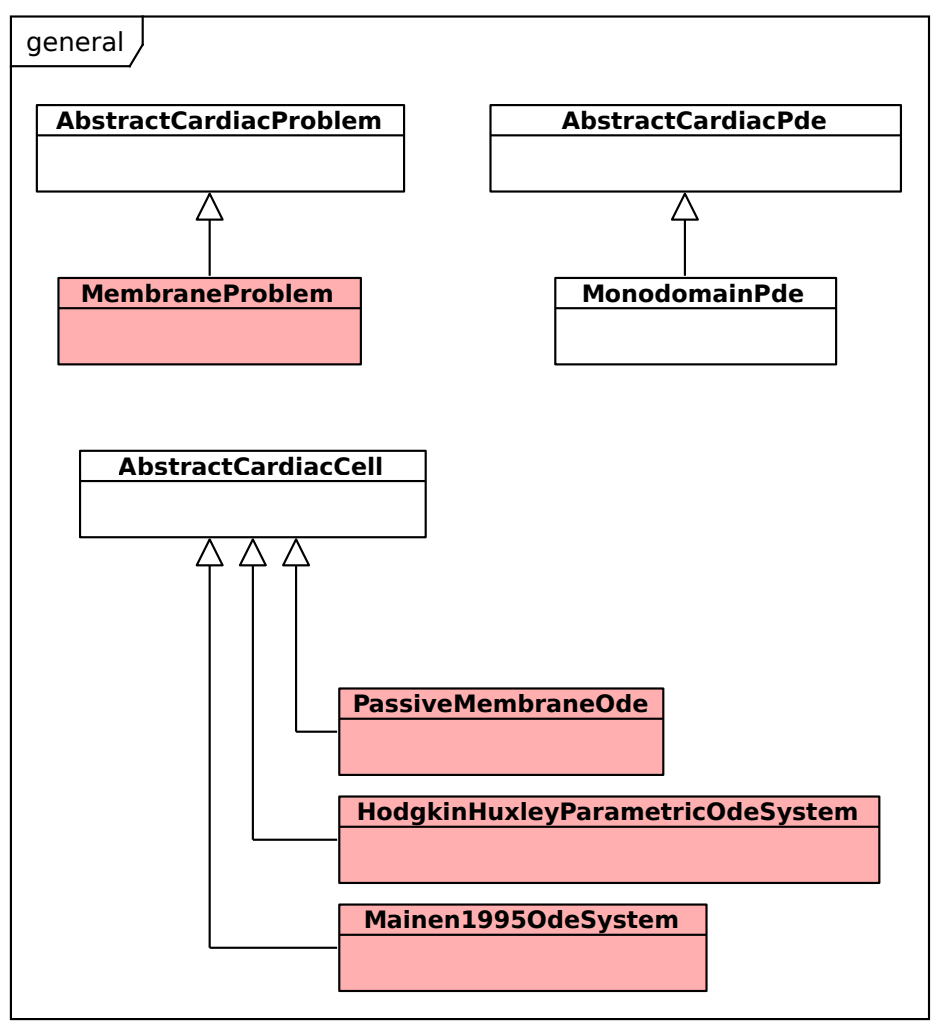

Figure A.1: General inheritance dependence of CHASTE-Membrane objects (light red) and CHASTE objects (white). 


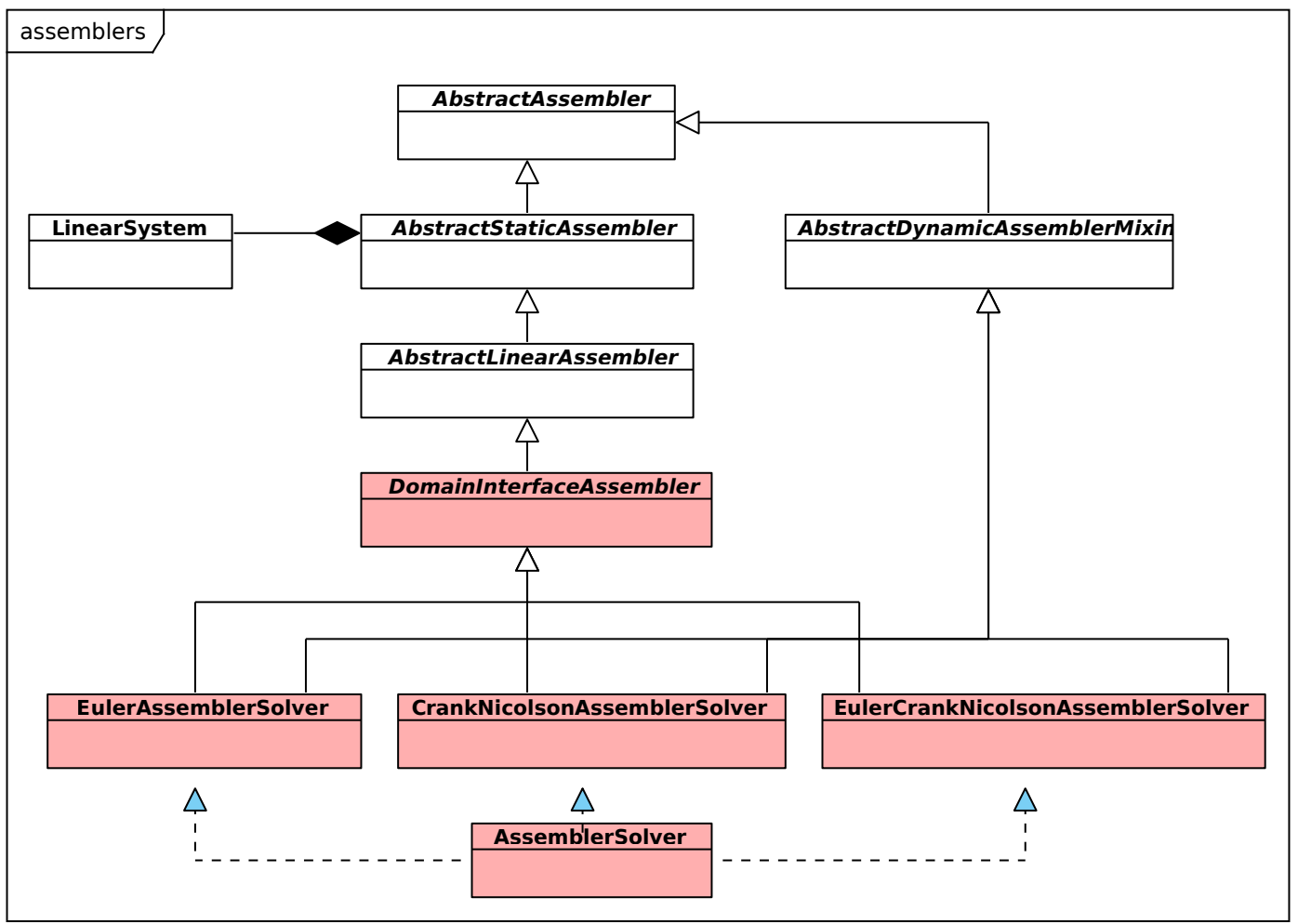

Figure A.2: Object inheritance dependence for the matrix assembly and solving objects of CHASTE-Membrane (light red) and CHASTE (white). 


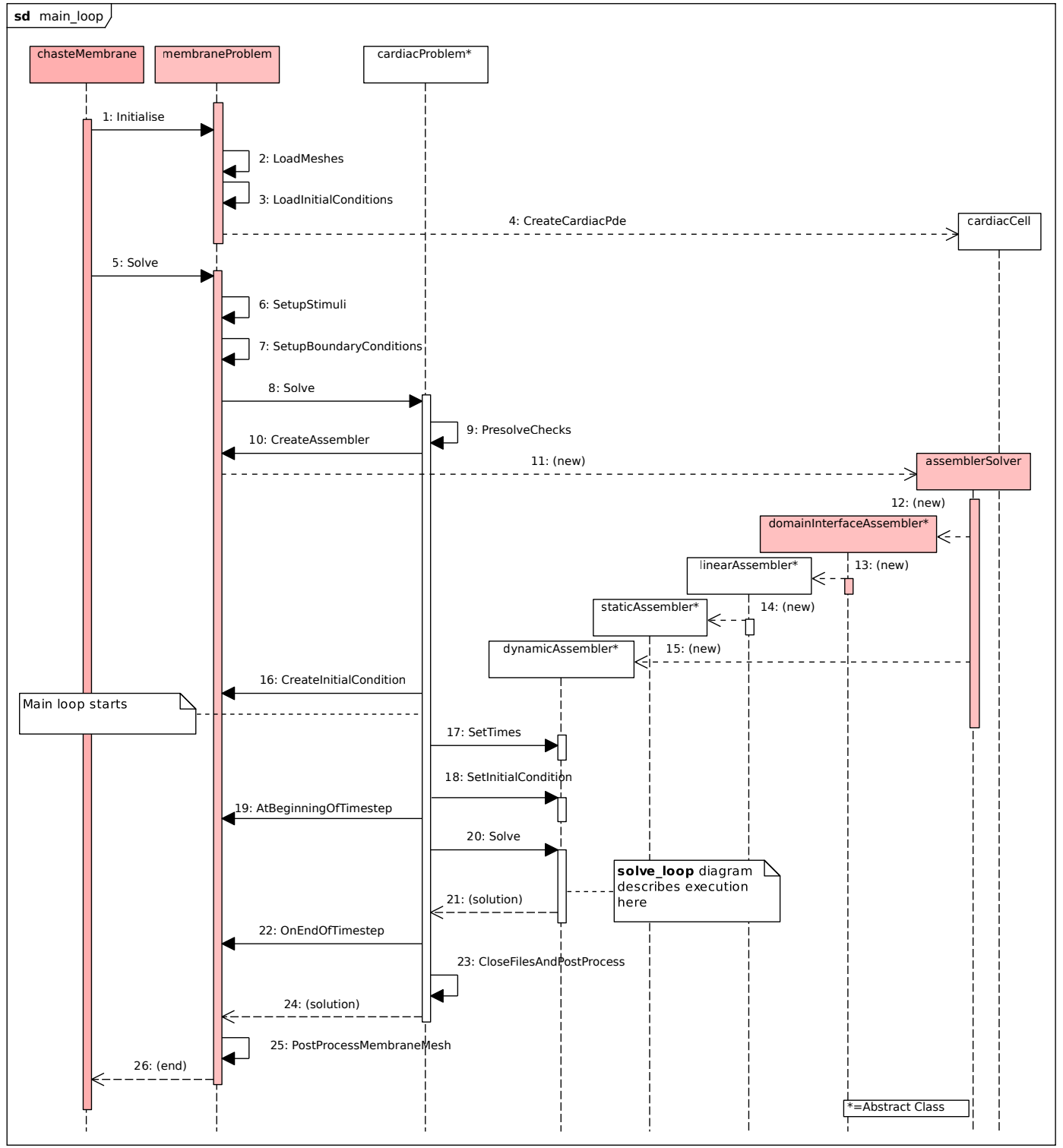

Figure A.3: Object sequential communication of CHASTE-Membrane objects (light red) and CHASTE objects (white) during the main program execution. 


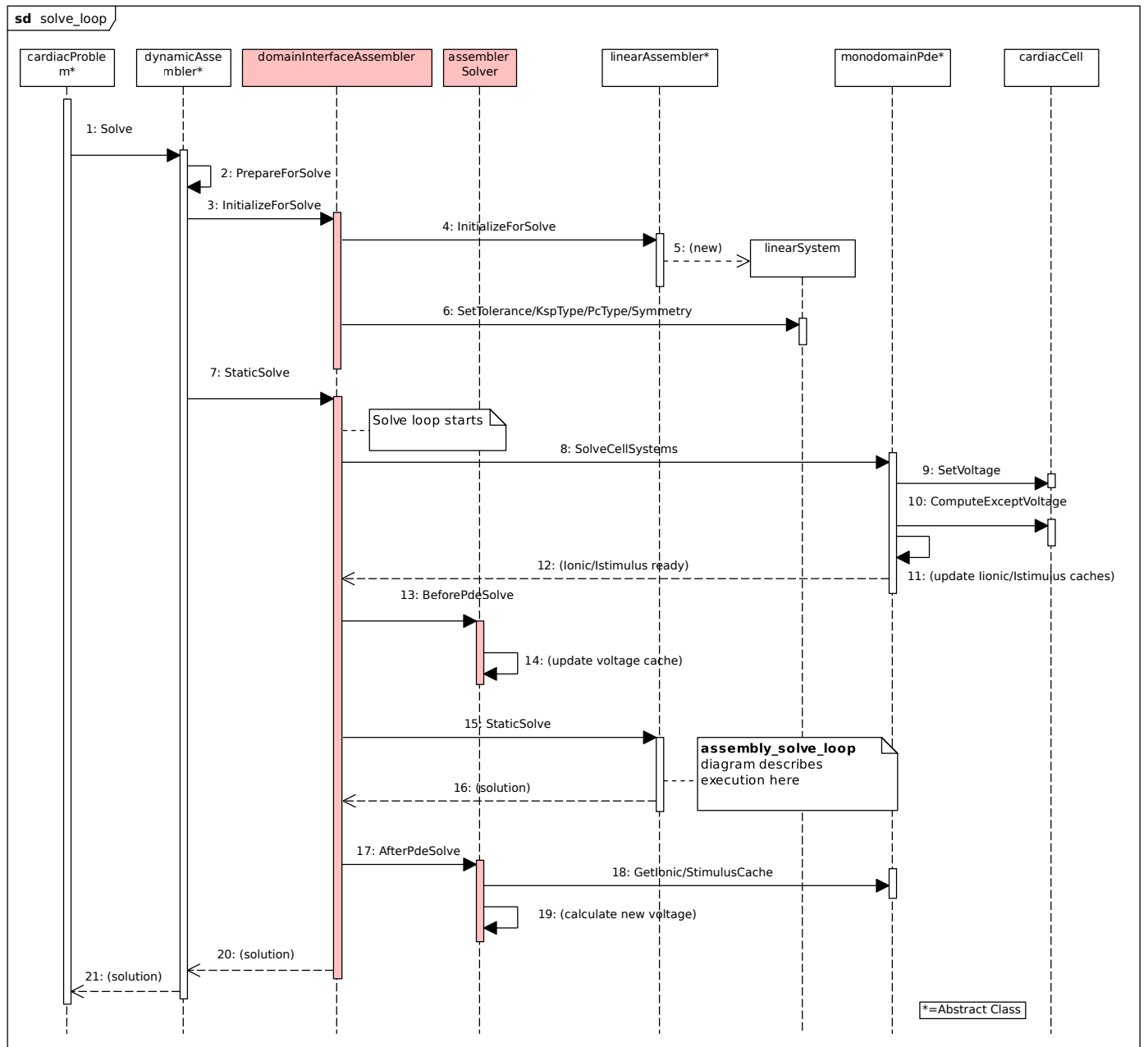

Figure A.4: Object sequential communication of CHASTE-Membrane objects (light red) and CHASTE objects (white) during the main solution loop. 


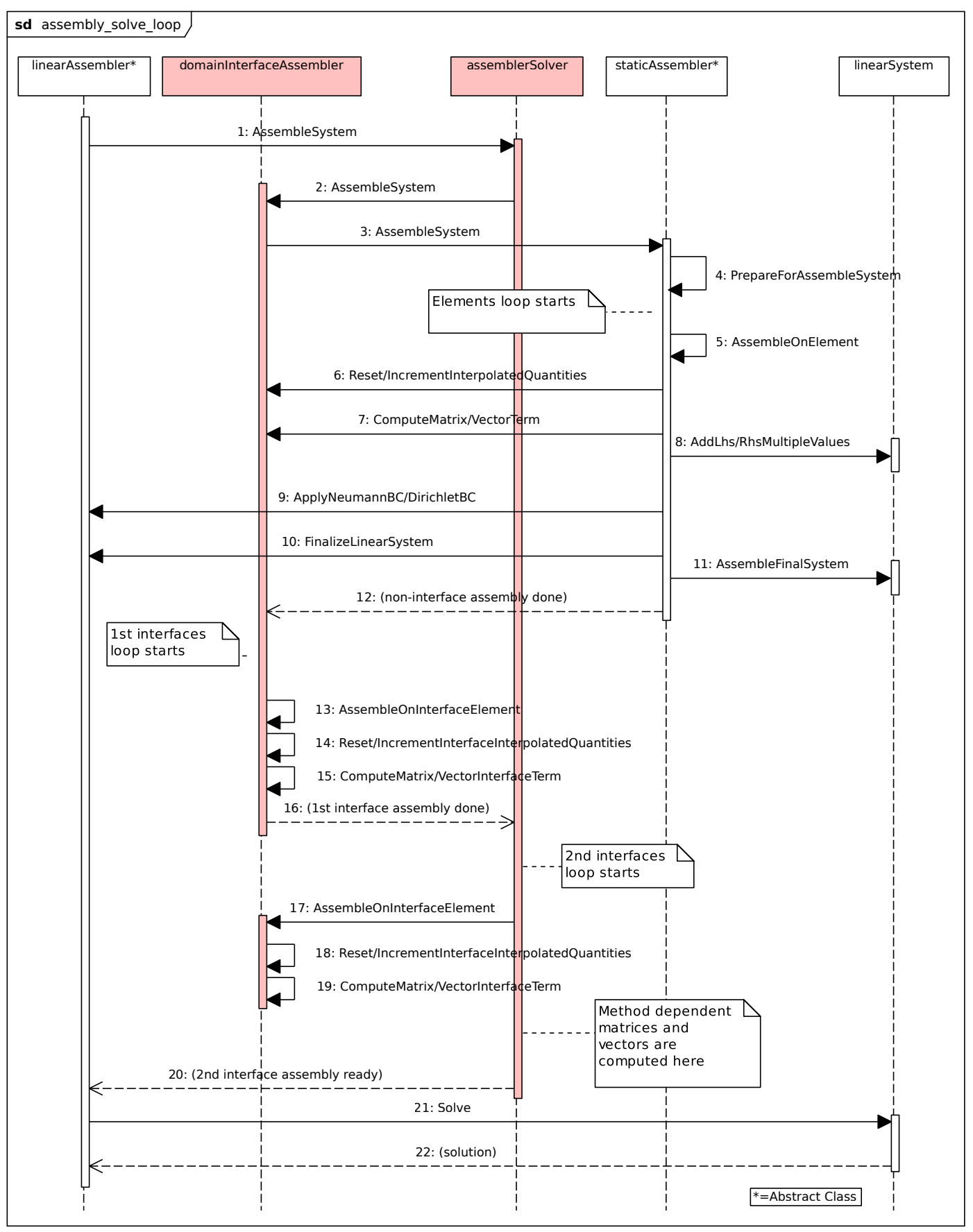

Figure A.5: Object sequential communication of CHASTE-Membrane objects (light red) and CHASTE objects (white) during the matrix assembly loop. 


\section{E 1D Cable Utility}

This chapter provides a guide for the cable simulation tool used to compare the numerical results of CHASTE-Membrane to the results of the cable equation. The tool implements the principles presented in Section 2.7.4.1. The tool was used for the analysis of Figures 3 and 4 of Chapter 7 and for the cable simulations of Chapter C. 
NRNSIM or NeuRoN SIMulation is a Python tool that facilitates the execution of simulations with the Neuron environment (http://WWW.neuron.yale.edu). NRNSIM uses Neuron's Python interface to provide access to Neuron. NRNSIM however facilitates the simulation of neurons by providing easy to use Python objects. NRNSIM is designed to extend Neuron with Python easily. NRNSIM provides one example application and this is for the simulation of the extracellular stimulation of neurons. This application can be configured with semi-arbitrary descriptions of electric fields changing in space and time and it will configure Neuron in such a way, that the current neuron geometry will respond as if it was inside this field. The electric fields and the type of Neuron mechanism for representation of the field in Neuron can be chosen as a Python object. At the moment, stimulation with Neuron IClamps and the 'extracellular' mechanism can be configured.

TOOL COMPONENTS

ニニニニニニニニニニニニニニ=

NRNSIM is composed by a collection of Python scripts. In the current version these scripts are:

-nsrun.py: This is the initialization script. This should be executed for every simulation. In this version the script should be manually edited to load the respective 'application' scripts. This script should always call nrnsim.py.

-nrnsim.py: The main script. This contains the basic functions most 'application' script should call, such as those to initialize Neuron and to set the default time parameters. The main functions are:

-settime(newdt, newtmax): Set time parameters in milliseconds. -nrngeom(name): Load the Neuron geometry given (.hoc file)

-nrninit(): Prepare Neuron for simulation

-updatemech(): Should be called if the Neuron mechanisms changed -sim(): initiates the Neuron simulation.

-section.py: Provides classes to wrap Neuron sections and section list providing a cleaner interface to obtain information about their parameters. The main functions are:

-Section.points(): returns a pair of 3D points with the start and end of a neuron section.

-Section.segments(): Retrieve all the pt3d defined segments in an array where each row is a point and a diameter.

-Section.dx(): Calculate the spatial differential of the section using the Neuron parameters of length and number of segments. 
-Section.setpoints(p๑,p1): Change the section initial and end coordinates.

-Section.ra(): Obtain the section's axial resistance in $0 \mathrm{hm} / \mathrm{m}$.

-Section.rm(): Obtain the section's membrane resistance in $\mathrm{Ohm}{ }^{*} \mathrm{~cm}$.

-Section.Ra(val): Set or get section's axial resistance in $\mathrm{Ohm}{ }^{*} \mathrm{~cm}$.

-Section.Rm(val): Set or get section's membrane resistance in $\mathrm{Ohm}^{*} \mathrm{Cm}^{\wedge} 2$.

-smech.py: Implements the NEURON calls of different methods for extra and some intracellular stimulation. Supported methods as Python objects are:
-ActivatingFunction: Implements the activation function [1] as a pair of stimulating IClamp objects in the two tips of each section. Current values are calculated as in $[2,3]$.
-ExtracellularMechanism: Uses Neuron's extracellular mechanism to set the extracellular potential for a set of sections. Each Neuron section is divided in segments. For every segment on every section, a potential value over time must be provided.
-CurrentClamp: A simple to use wrapper for Neuron's IClamp mechanism.

-fields.py: Provides objects for signals and fields. A signal is a variable depending on time. A field is a $3 d$ variable depending on time defined for a set of points. Classes provided are:

-StepSignal: Given a time vector and a start and end generates a time series in a vector that resembles a step function.

-BipolarSignal: A square bipolar function where a start, switching time and end time should be provided.

-CosSignal: A cosine signal with and amplitude, period, start of the signal and end of the signal.

-RLCSignal: Emulates the electric field induced by the inductor in a Resistor, Inductor, Capacitor circuit. It emulates the electric field produced by a transcranial magnetic stimulation coil.

-Field: A general class to represent a field. Field objects can take as input a signal object to scale them over time.

-HomogField: produces a spatially homogeneous field but variable in time field for a given time changing signal.

EXAMPLE APPLICATIONS

=ニニニニニニ=ニニニニニ=ニニ=ニ=ニ

One example application script is provided with NRNSIM:

-multi.py: Simulates multiple types of 1D neural geometries, under 
geometrical transformations and multiple stimulation methods. The file multi.py can be used as a template for other applications. multi.py provides a graphical user interface to change the magnitude of and duration of the stimulation field. On execution of nsrun.py, parameters can be given in the command line to load an specific hoc file and an extra Python script. This script can modify the default amplitude, duration, orientation of the field and timesteps.

TOOL USAGE

ニニニニニニニニニニ

NRNSIM requires that Neuron is installed with Python support and that Neuron is visible to the Python interpreter, this is explained in

http://www. neuron.yale.edu/neuron/download

For execution the regular Python interpreter can be used but IPython is recommended. To run the default multi.py application with IPython $<0.11$ use the command:

ipython -pylab -i -c '\%run -i nsrun.py file'

Where file is the root name of a set of files:

file.hoc: a Neuron geometry. Cell parameters can also be set.

file.ses: a Neuron session file.

file.py: a configuration file with the stimulation parameters.

file.cin.hoc: alternatively, a file with hoc's init() function. This

can be used to set arbitrary initial potentials

for different cells.

IPython $\odot .11$ and superior changed the interaction of IPython with QT gui (which is required by the tool). To use IPython then using a separate kernel is recommended to do this call instead:

ipython console --pylab qt -i -c "\%run -i nsrun.py file"

alternatively simple IPython can be used (no kernel mode) calling:

ipython -i -c "\%run -i nsrun.py file"

but in this case IPython's plotting routines cannot be used.

\section{REFERENCES}

[1] Frank Rattay. Analysis of models for external stimulation of axons. IEEE Transactions on Biomedical Engineering, 33(10): $974-977,1986$.

[2] S. S. Nagarajan, D. M. Durand, and E. N. Warman. Efects of induced electric filds on finite neuronal structures: a simulation study. IEEE transactions on bio-medical engineering, 40(11):1175-1188, 1993.

[3] Assaf Rotem and Elisha Moses. Magnetic stimulation of one-dimensional neuronal cultures. Biophysical Journal, 94(12): 5065-5078, 2008. 


\section{F Coil Simulation Tool}

The following guide covers the numerical simulation tool used to calculate the fields produced by realistic transcranial magnetic stimulation coils in Chapter C. 
COIL TOOL USER GUIDE

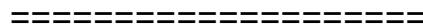

$\checkmark 0.312 .2 .2010$

$\checkmark 0.214 .8 .2009$

$\mathrm{V} 0.127 .2 .2009$

Coil Tool is a numerical integrator for calculating the electric field induced by coils, typically those used in transcranial magnetic stimulation. The tool can calculate the electric field over a range of evenly spaced coordinates in three-dimensions. The field is calculated from a linear piecewise description of the coil. The tool is written in the $C$ language but can be accessed from Python via dynamic link library wrappers.

TOOL INTERNALS

ニニニニニニニニニニニニニニ

The tool is divided in two basic modules: a module that produces coil geometries (coilgen) and a module that calculates the electric field via the magnetic vector potential (mvp). The tool implements equation (4) in the paper of Salinas et al [1] in the form:

$$
E(r)=-\left(m u \odot / 4{ }^{*} P I\right) * d I / d t * \text { integral }(d l /(r-r \odot))
$$

The calculation is time independent and assumes the current time derivative is always $d I / d t=1$. The results should be properly scaled by this value and over time.

The coil should be described in *.csv file as a list of consecutive points $x, y, z$ in meters. Point $\mathrm{n}$ to point $\mathrm{n}+1$ describe the $\mathrm{dl} \mathrm{n}$ element.

The output is a list of $x, y, z, E x, E y, E z$ values describing the result of the main integral integral for each of the points in meters specified in the solution range.

THE COILGEN MODULE

ニニニニニニニニニニニニニニニニニニ

The coilgen module can be used to produce four types of parametrized coils or to load coils specified with *.csv files in other tools. Any coil for which the electric field is calculated must be loaded in memory first by the coilgen tool and then passed to the mvp module to produce the final calculation. The set of predefined and parametrized coil tools are:

* Circular coil: A single circular coil. The radius of the coil should be specified and the length of the differential dl.

* Spiral coil: The coil internal and external radii, the number of loops and the length differential.

* Water drop coil: A coil shaped like a water drop, with a sharp angle at one end

loops,

differential and a curve at the other end. For this coil the number of the radius of the curved end, the length from the center to the sharp end, the angle of the sharp end and the length

should be specified.

* Fourier coil: A spiral coil shaped like a petal that uses the three first 
sin/cos terms of a Fourier series to produce the form. The Fourier coefficients should be specified, together with the number of loops, a radius increase differential, the outer radius and the length differential.

The details of the parameters can be seen in the corresponding source code files: circular_coil.c, spiral_coil.c, waterdrop_coil.c and fourier_coil.c.

The coil routines always generate a single leaf of a TMS coil. For more complicated geometries (e.g. figure of eight coils) coil groups should be used. coil groups simply create duplicates of coil objects and generates complex configurations of them, providing several layers for the coil or pairs of them. The four types of configurations supported are:

${ }^{*}$ coil_group_1: single coil leaf but with several z layers

${ }^{*}$ coil_group_2_x: two leafs with $\mathrm{z}$ layers in the $\mathrm{x}$ axis

*coil_group_2_y: two leafs with $z$ layers in the y axis

${ }^{*}$ coil_group_4: four leafs with $z$ layers in the $x$ and $y$ axis

Coil coordinates in coil groups are always transformed so that the hotspot of the coil is in the $\odot, \Theta, \Theta$ coordinate.

Description of the parameters for this functions can be seen in: coilgenmodule.c

Although $\mathrm{C}$ code can be used to build or load the coil, the Python interface is recommended. To use the coilgen module the shared libraries should be first build in Linux using the script build.bash and then in Python:

import imp

coilgen = imp.load_dynamic ('coilgen', './libcoilgen.so' $)$

where ./libcoilgen.so should be replaced with the path where the shared library was built. After this coil objects can be used simply by calling the respective function, for example:

ccoil1mm = coilgen. circular $(0.04,0.0 .001)$

builds a circular coil of radius $4 \mathrm{~cm}$ with length differentials of $1 \mathrm{~mm}$.

A coil group must then be created by calling for example:

ccoil1mmg = coilgen.coil_group_1 $(\operatorname{ccoil} 1 \mathrm{~mm})$

Example for usage of all the coil types is given in the files *analysis.py where * is any of the coil types. Usage of the *.csv loading of coils is shown in idepclover_analysis.py. The function coilgen.load_coil(filename) should be used for this.

\section{THE MVP MODULE}

The mvp module calculates the electric field and takes as input a coil group and a 3D cubic domain of solution. The main function to be called is

$$
\text { calculate_coil_field(cg, xl,xh,yl,yh,zl,zh, dx, file) }
$$

The parameters are: 
${ }^{*} \mathrm{cg}$ : the coil group

${ }^{*} \mathrm{xl}, \mathrm{xh}$ : the low and high $\mathrm{x}$ range for the domain.

${ }^{*} \mathrm{yl}, \mathrm{yh}$ : the low and high y range for the domain.

${ }^{*} \mathrm{zl}, \mathrm{zh}$ : the low and high $\mathrm{z}$ range for the domain.

*file: the name of the *.csv file where the output will be put.

The $C$ specification of this function can be seen in the file mvpmodule.c.

As with the coilgen module, the mvp module can be used from python. To use the coilgen module the shared libraries should be first build in Linux using the script build.bash and then in Python:

import imp

mvp = imp.load_dynamic( 'mvp', './libmvp.so')

where ./libmvp.so should be replaced with the path where the shared library was built. After the fields can be calculated simply by calling for example:

mvp.calculate_coil_field(ccoil1mmg, - $0.05,0.05,-0.05,0.05,0.01,0.01,0.001$, '..././data/coilfields/cE1mm.csv')

which will use the $1 \mathrm{~mm}$ circular coil group to calculate the electric field in a $10 \times 10 \times 2 \mathrm{~cm}$ cube.

Usage of the mvp module is shown for example in idepclover_analysis.py and in convergence_analysis.py.

\section{REFERENCES}

$===ニ==ニ==$

[1] Salinas FS, Lancaster JL, Fox PT. Detailed 3D models of the induced electric field of transcranial magnetic stimulation coils. Physics in medicine and biology. 2007 May;52(10):2879-2892. 


\section{G Solution Algorithms}

This appendix presents an algorithm form of the numerical solution methods presented in Chapter 4. These algorithms provide an accessible summary of the methods implemented in the tool of Chapter 6. The algorithms are functional and conveniently written in the Python scripting language [127]. 


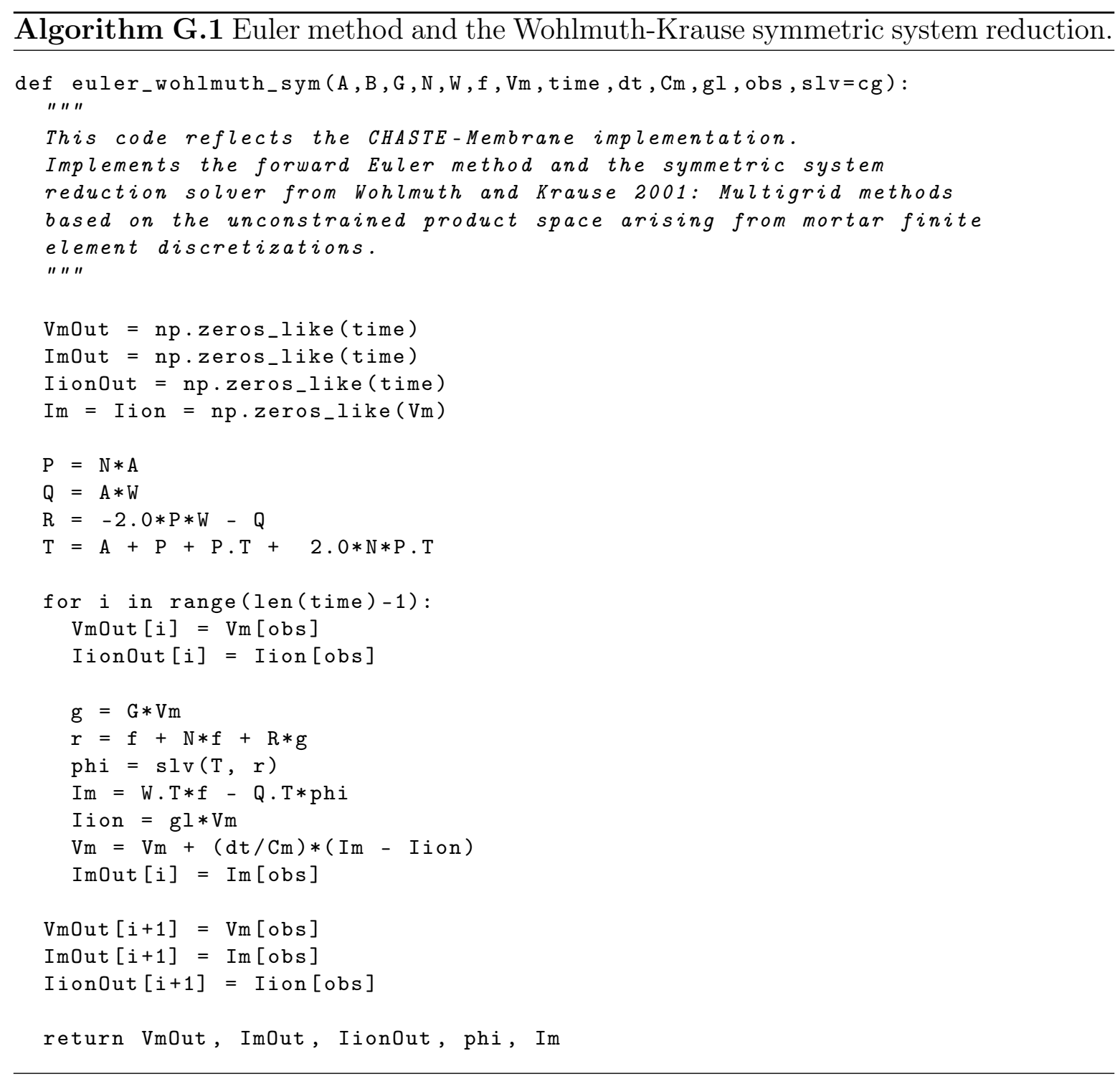




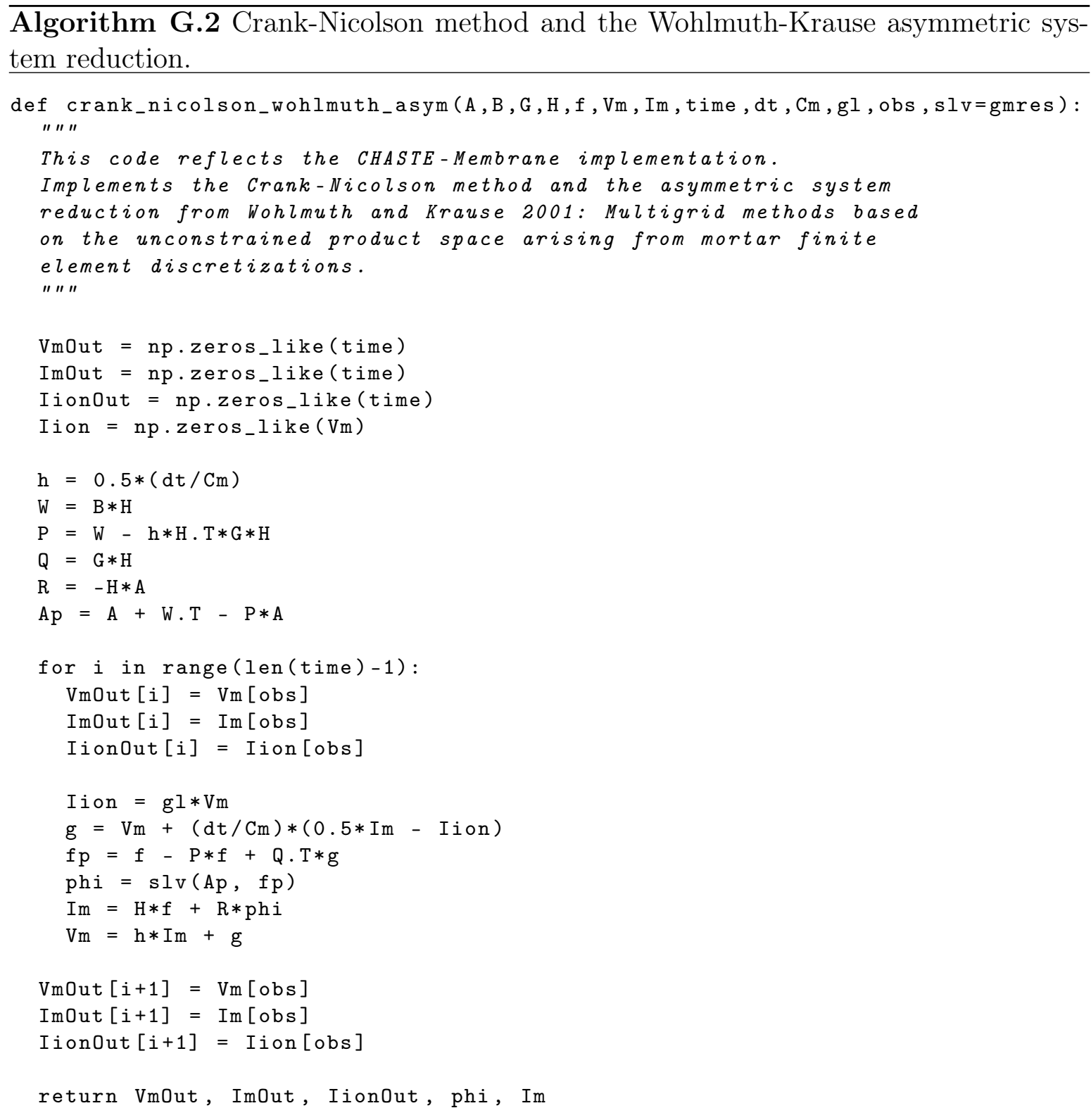




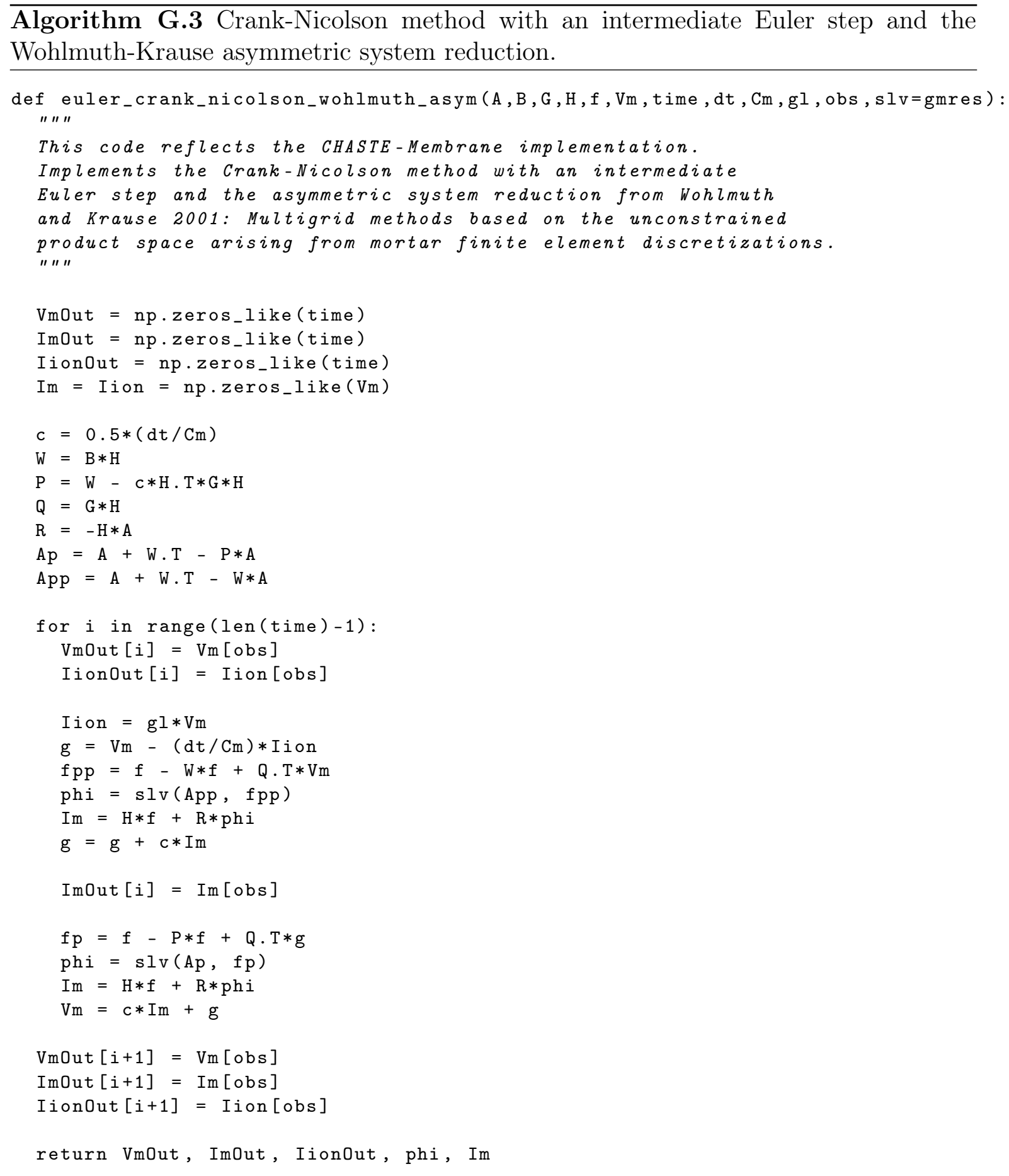




\section{Bibliography}

[1] Winfried Denk and Heinz Horstmann. Serial Block-Face Scanning Electron Microscopy to Reconstruct Three-Dimensional Tissue Nanostructure. PLoS Biol, 2(11):e329+, October 2004.

[2] Viren Jain, Sebastian S. Seung, and Srinivas C. Turaga. Machines that learn to segment images: a crucial technology for connectomics. Current opinion in neurobiology, 20(5):653-666, October 2010.

[3] A. L. Hodgkin and A. F. Huxley. A quantitative description of membrane current and its application to conduction and excitation in nerve. The Journal of Physiology, 117(4):500-544, August 1952.

[4] W. Rall. Theory of physiological properties of dendrites. Ann NYAcad Sci, 96:1071-1092, March 1962.

[5] Eva Syková and Charles Nicholson. Diffusion in Brain Extracellular Space. Physiological Reviews, 88(4):1277-1340, October 2008.

[6] Robert G. Thorne and Charles Nicholson. In vivo diffusion analysis with quantum dots and dextrans predicts the width of brain extracellular space. Proceedings of the National Academy of Sciences, 103(14):5567-5572, April 2006.

[7] K. J. Hayworth. Automatic taping lathe-microtome. In Proceedings of the Southern California Society for Microscopy and Microanalysis, April 2005.

[8] Costas A. Anastassiou, Rodrigo Perin, Henry Markram, and Christof Koch. Ephaptic coupling of cortical neurons. Nature neuroscience, 14(2):217-223, February 2011.

[9] Flavio Fröhlich and David A. McCormick. Endogenous electric fields may guide neocortical network activity. Neuron, 67(1):129-143, July 2010.

[10] Simal Ozen, Anton Sirota, Mariano A. Belluscio, Costas A. Anastassiou, Eran Stark, Christof Koch, and György Buzsáki. Transcranial electric stimulation entrains cortical neuronal populations in rats. The Journal of neuroscience : the official journal of the Society for Neuroscience, 30(34):11476-11485, August 2010.

[11] James Kozloski and John Wagner. An Ultrascalable Solution to Large-scale Neural Tissue Simulation. Frontiers in neuroinformatics, 5, 2011. 
[12] M. L. Hines and N. T. Carnevale. The NEURON Simulation Environment. Neural Computation, 9(6):1179-1209, August 1997.

[13] Sarah H. Lisanby, Thomas E. Schlaepfer, Hans-Ulrich Fisch, and Harold A. Sackeim. Magnetic Seizure Therapy of Major Depression. Arch Gen Psychiatry, 58(3):303-305, March 2001.

[14] Mark Hallett. Transcranial Magnetic Stimulation: A Primer. Neuron, 55(2):187199, July 2007.

[15] R. E. Gross and A. M. Lozano. Advances in neurostimulation for movement disorders. Neurological research, 22(3):247-258, April 2000.

[16] F. Sun, M. Morrell, and R. Wharenjr. Responsive Cortical Stimulation for the Treatment of Epilepsy. Neurotherapeutics, 5(1):68-74, January 2008.

[17] M. A. Nitsche and W. Paulus. Excitability changes induced in the human motor cortex by weak transcranial direct current stimulation. The Journal of physiology, 527 Pt 3(3):633-639, September 2000.

[18] Ryota Kanai, Leila Chaieb, Andrea Antal, Vincent Walsh, and Walter Paulus. Frequency-dependent electrical stimulation of the visual cortex. Current biology : $C B, 18(23): 1839-1843$, December 2008.

[19] Tamás Z. Kincses, Andrea Antal, Michael A. Nitsche, Orsolya Bártfai, and Walter Paulus. Facilitation of probabilistic classification learning by transcranial direct current stimulation of the prefrontal cortex in the human. Neuropsychologia, 42(1):113-117, January 2004.

[20] Felipe Fregni, Paulo S. Boggio, Michael Nitsche, Felix Bermpohl, Andrea Antal, Eva Feredoes, Marco A. Marcolin, Sergio P. Rigonatti, Maria T. A. Silva, Walter Paulus, and Alvaro Pascual-Leone. Anodal transcranial direct current stimulation of prefrontal cortex enhances working memory. Experimental Brain Research, 166(1):23-30, September 2005.

[21] Agnes Flöel, Nina Rösser, Olesya Michka, Stefan Knecht, and Caterina Breitenstein. Noninvasive Brain Stimulation Improves Language Learning. Journal of Cognitive Neuroscience, 20(8):1415-1422, February 2008.

[22] Scott F. Lempka, Matthew D. Johnson, Michael A. Moffitt, Kevin J. Otto, Daryl R. Kipke, and Cameron C. McIntyre. Theoretical analysis of intracortical microelectrode recordings. Journal of neural engineering, 8(4):045006+, August 2011.

[23] Tianhe C. Zhang and Warren M. Grill. Modeling deep brain stimulation: point source approximation versus realistic representation of the electrode. Journal of Neural Engineering, 7(6):066009+, December 2010. 
[24] E. J. Peterson, O. Izad, and D. J. Tyler. Predicting myelinated axon activation using spatial characteristics of the extracellular field. Journal of neural engineering, 8(4):046030+, August 2011.

[25] H. G. Meijer, M. Krupa, H. Cagnan, M. A. Lourens, T. Heida, H. C. Martens, L. J. Bour, and S. A. van Gils. From Parkinsonian thalamic activity to restoring thalamic relay using deep brain stimulation: new insights from computational modeling. Journal of neural engineering, 8(6), December 2011.

[26] M. Djilas, C. Olès, H. Lorach, A. Bendali, J. Dégardin, E. Dubus, G. LissorguesBazin, L. Rousseau, R. Benosman, S. H. Ieng, S. Joucla, B. Yvert, P. Bergonzo, J. Sahel, and S. Picaud. Three-dimensional electrode arrays for retinal prostheses: modeling, geometry optimization and experimental validation. Journal of Neural Engineering, 8(4):046020+, June 2011.

[27] Ling Wang, Michael Riss, Jennifer Olmos O. Buitrago, and Enric ClaverolTinturé. Biophysics of microchannel-enabled neuron-electrode interfaces. Journal of neural engineering, 9(2), April 2012.

[28] Hsin-Yi Y. Lai, Lun-De D. Liao, Chin-Teng T. Lin, Jui-Hsiang H. Hsu, Xin He, You-Yin Y. Chen, Jyh-Yeong Y. Chang, Hui-Fen F. Chen, Siny Tsang, and Yen$\mathrm{Yu}$ Y. Shih. Design, simulation and experimental validation of a novel flexible neural probe for deep brain stimulation and multichannel recording. Journal of neural engineering, 9(3), June 2012.

[29] James J. FitzGerald, Natalia Lago, Samia Benmerah, Jordi Serra, Christopher P. Watling, Ruth E. Cameron, Edward Tarte, Stéphanie P. Lacour, Stephen B. McMahon, and James W. Fawcett. A regenerative microchannel neural interface for recording from and stimulating peripheral axons in vivo. Journal of Neural Engineering, 9(1):016010+, January 2012.

[30] György Buzsáki, Costas A. Anastassiou, and Christof Koch. The origin of extracellular fields and currents - EEG, ECoG, LFP and spikes. Nat Rev Neurosci, 13(6):407-420, May 2012.

[31] György Buzsáki. Large-scale recording of neuronal ensembles. Nature neuroscience, 7(5):446-451, May 2004.

[32] Henrik Lindén, Tom Tetzlaff, Tobias C. Potjans, Klas H. Pettersen, Sonja Grün, Markus Diesmann, and Gaute T. Einevoll. Modeling the Spatial Reach of the LFP. Neuron, 72(5):859-872, December 2011.

[33] Claude Bédard, Helmut Kröger, and Alain Destexhe. Modeling Extracellular Field Potentials and the Frequency-Filtering Properties of Extracellular Space. Biophysical Journal, 86(3):1829-1842, March 2004.

[34] Frank Rattay. Analysis of models for external stimulation of axons. IEEE Transactions on Biomedical Engineering, 33(10):974-977, 1986. 
[35] E. C. Fear and M. A. Stuchly. Modeling assemblies of biological cells exposed to electric fields. IEEE transactions on bio-medical engineering, 45(10):1259-1271, October 1998.

[36] Gary R. Holt and Christof Koch. Electrical Interactions via the Extracellular Potential Near Cell Bodies. Journal of Computational Neuroscience, 6(2):169184, March 1999.

[37] Wenjun Ying and Craig S. Henriquez. Hybrid Finite Element Method for Describing the Electrical Response of Biological Cells to Applied Fields. IEEE Transactions on Biomedical Engineering, 54(4):611-620, April 2007.

[38] Christine Voßen, Jens Eberhard, and Gabriel Wittum. Modeling and simulation for three-dimensional signal propagation in passive dendrites. Computing and Visualization in Science, 10(2):107-121, June 2007.

[39] Céline Moulin, Alain Glière, Daniel Barbier, Sebastien Joucla, Blaise Yvert, Pascal Mailley, and Régis Guillemaud. A new 3-D finite-element model based on thin-film approximation for microelectrode array recording of extracellular action potential. IEEE transactions on bio-medical engineering, 55(2 Pt 1):683-692, February 2008.

[40] Carl Gold, Darrell A. Henze, Christof Koch, and György Buzsáki. On the Origin of the Extracellular Action Potential Waveform: A Modeling Study. Journal of Neurophysiology, 95(5):3113-3128, May 2006.

[41] Klas H. Pettersen and Gaute T. Einevoll. Amplitude variability and extracellular low-pass filtering of neuronal spikes. Biophysical journal, 94(3):784-802, February 2008 .

[42] R. Plonsey. The nature of sources of bioelectric and biomagnetic fields. Biophysical journal, 39(3):309-312, September 1982.

[43] Stefan Luther, Flavio H. Fenton, Bruce G. Kornreich, Amgad Squires, Philip Bittihn, Daniel Hornung, Markus Zabel, James Flanders, Andrea Gladuli, Luis Campoy, Elizabeth M. Cherry, Gisa Luther, Gerd Hasenfuss, Valentin I. Krinsky, Alain Pumir, Robert F. Gilmour, and Eberhard Bodenschatz. Low-energy control of electrical turbulence in the heart. Nature, 475(7355):235-239, July 2011.

[44] B. J. Roth and P. J. Basser. A model of the stimulation of a nerve fiber by electromagnetic induction. IEEE transactions on bio-medical engineering, 37(6):588597, June 1990.

[45] S. S. Nagarajan, D. M. Durand, and E. N. Warman. Effects of induced electric fields on finite neuronal structures: a simulation study. IEEE transactions on bio-medical engineering, 40(11):1175-1188, November 1993.

[46] Cameron C. McIntyre, Warren M. Grill, David L. Sherman, and Nitish V. Thakor. Cellular Effects of Deep Brain Stimulation: Model-Based Analysis of Activation and Inhibition. J Neurophysiol, 91(4):1457-1469, April 2004. 
[47] Robert Plonsey and Roger C. Barr. Bioelectricity: A Quantitative Approach. Springer, 3rd edition, June 2007.

[48] Sébastien Joucla and Blaise Yvert. Improved Focalization of Electrical Microstimulation Using Microelectrode Arrays: A Modeling Study. PLoS ONE, 4(3):e4828+, March 2009.

[49] K. W. Altman and R. Plonsey. Analysis of excitable cell activation: relative effects of external electrical stimuli. Medical \& Biological Engineering $\&$ Computing, 28(6):574-580, November 1990.

[50] S. Joucla and B. Yvert. The "mirror" estimate: an intuitive predictor of membrane polarization during extracellular stimulation. Biophysical journal, 96(9):34953508, May 2009.

[51] C. H. See, R. A. Abd-Alhameed, and P. S. Excell. Computation of Electromagnetic Fields in Assemblages of Biological Cells Using a Modified Finite-Difference Time-Domain Scheme. Microwave Theory and Techniques, IEEE Transactions on, 55(9):1986-1994, 2007.

[52] Gorazd Pucihar, Damijan Miklavcic, and Tadej Kotnik. A time-dependent numerical model of transmembrane voltage inducement and electroporation of irregularly shaped cells. IEEE transactions on bio-medical engineering, 56(5):14911501, May 2009.

[53] Konstantinos Xylouris, Gillian Queisser, and Gabriel Wittum. A threedimensional mathematical model of active signal processing in axons. Comput. Vis. Sci., 13(8):409-418, December 2010.

[54] E. J. Vigmond, R. Weber dos Santos, A. J. Prassl, M. Deo, and G. Plank. Solvers for the cardiac bidomain equations. Progress in biophysics and molecular biology, 96(1-3):3-18, January 2008.

[55] J. Crank and P. Nicolson. A Practical Method for Numerical Evaluation of Solutions of Partial Differential Equations of the Heat Conduction Type. Proc. Cam. phil. Soc., 43:50-64, 1947.

[56] William Thomson. On the Theory of the Electric Telegraph. Proceedings of the Royal Society of London, 7, 1854.

[57] J. Clerk Maxwell. A Dynamical Theory of the Electromagnetic Field. Philosophical Transactions of the Royal Society of London, 155:459-512, 1865.

[58] J Bernstein. Ueber den zeitlichen verlauf der negativen schwankung des nervenstroms. Pflügers Archiv European Journal of Physiology, 1(1):173-207, 1868.

[59] Robert Plonsey. Volume Conductor Fields of Action Currents. Biophys. J., 4(4):317-328, July 1964. 
[60] L. A. Cartee and R. Plonsey. The transient subthreshold response of spherical and cylindrical cell models to extracellular stimulation. IEEE transactions on bio-medical engineering, 39(1):76-85, January 1992.

[61] K. Lindsay. From Maxwell's equations to the cable equation and beyond. Progress in Biophysics and Molecular Biology, 85(1):71-116, May 2004.

[62] Paul L. Nunez and Ramesh Srinivasan. Electric Fields of the Brain: The Neurophysics of EEG, 2nd Edition. Oxford University Press, USA, 2 edition, December 2005.

[63] John D. Jackson. Classical Electrodynamics Third Edition. Wiley, third edition, August 1998.

[64] David J. Griffiths. Introduction to Electrodynamics. Prentice Hall, 2nd edition, 1989.

[65] Robert Plonsey and Dennis Heppner. Considerations of quasi-stationarity in electrophysiological systems. Bulletin of Mathematical Biology, 29(4):657-664, December 1967.

[66] R. Plonsey. Volume Conductor Theory. CRC Press LLC, 2000.

[67] Peter Dayan and L. F. Abbott. Theoretical Neuroscience: Computational and Mathematical Modeling of Neural Systems. The MIT Press, 1st edition, December 2001.

[68] Idan Segev. Cable and Compartmental Models of Dendritic Trees. Springer-Verlag New York, Inc., New York, NY, USA, 1998.

[69] Henry C. Tuckwell. Introduction to Theoretical Neurobiology: Volume 1, Linear Cable Theory and Dendritic Structure, volume 1. 1988.

[70] Wulfram Gerstner and Werner M. Kistler. Spiking Neuron Models: Single Neurons, Populations, Plasticity. Cambridge University Press, 1 edition, August 2002.

[71] Barbara I. Wohlmuth and Rolf H. Krause. A Multigrid Method Based on the Unconstrained Product Space for Mortar Finite Element Discretizations. SIAM J. Numer. Anal., 39(1):192-213, January 2001.

[72] John D. Jackson. Classical Electrodynamics. John Wiley \& Sons, 1st edition, 1962.

[73] Assaf Rotem and Elisha Moses. Magnetic stimulation of one-dimensional neuronal cultures. Biophysical journal, 94(12):5065-5078, June 2008.

[74] Hiromu Monai, Toshiaki Omori, Masato Okada, Masashi Inoue, Hiroyoshi Miyakawa, and Toru Aonishi. An analytic solution of the cable equation predicts frequency preference of a passive shunt-end cylindrical cable in response to extracellular oscillating electric fields. Biophysical journal, 98(4):524-533, February 2010. 
[75] W. Krassowska and J. C. Neu. Response of a single cell to an external electric field. Biophys J, 66(6):1768-1776, June 1994.

[76] Tadej Kotnik, Damijan Miklavčič, and Tomaž Slivnik. Time course of transmembrane voltage induced by time-varying electric fields - a method for theoretical analysis and its application. Bioelectrochemistry and Bioenergetics, 45(1):3-16, March 1998.

[77] G. W. Beeler and H. Reuter. Reconstruction of the action potential of ventricular myocardial fibres. The Journal of physiology, 268(1):177-210, June 1977.

[78] Hermann A. Haus and James R. Melcher. Electromagnetic Fields and Energy. Prentice Hall, January 1989.

[79] C. S. Henriquez. Simulating the electrical behavior of cardiac tissue using the bidomain model. Critical reviews in biomedical engineering, 21(1):1-77, 1993.

[80] Glenn T. Lines, Per Grottum, and Aslak Tveito. Modeling the electrical activity of the heart: A Bidomain Model of the ventricles embedded in a torso. Computing and Visualization in Science, 5(4):195-213, April 2003.

[81] E. C. Fear and M. A. Stuchly. A novel equivalent circuit model for gap-connected cells. Physics in Medicine and Biology, 43(6):1439+, June 1998.

[82] Airton Ramos, Adroaldo Raizer, and Jefferson L. Marques. A new computational approach for electrical analysis of biological tissues. Bioelectrochemistry (Amsterdam, Netherlands), 59(1-2):73-84, April 2003.

[83] D. A. Stewart, T. R. Gowrishankar, K. C. Smith, and J. C. Weaver. Cylindrical cell membranes in uniform applied electric fields: validation of a transport lattice method. Biomedical Engineering, IEEE Transactions on, 52(10):1643-1653, October 2005.

[84] Donald A. Stewart, T. R. Gowrishankar, and James C. Weaver. Three dimensional transport lattice model for describing action potentials in axons stimulated by external electrodes. Bioelectrochemistry, 69(1):88-93, September 2006.

[85] L. J. Leon and F. A. Roberge. A model study of extracellular stimulation of cardiac cells. Biomedical Engineering, IEEE Transactions on, 40(12):1307-1319, December 1993.

[86] K. R. Foster and A. E. Sowers. Dielectrophoretic forces and potentials induced on pairs of cells in an electric field. Biophysical journal, 69(3):777-784, September 1995 .

[87] Dejan Šemrov and Damijan Miklavčič. Numerical Modeling for In Vivo Electroporation. In Mark J. Jaroszeski, Richard Heller, Richard Gilbert, John M. Walker, and John M. Walker, editors, Electrochemotherapy, Electrogenetherapy, and Transdermal Drug Delivery, volume 37 of Methods in Molecular Medicine, pages 63-81. Humana Press, New Jersey, February 2000. 
[88] Mojca Pavlin, Natasa Pavselj, and Damijan Miklavcic. Dependence of induced transmembrane potential on cell density, arrangement, and cell position inside a cell system. IEEE transactions on bio-medical engineering, 49(6):605-612, June 2002.

[89] K. Xylouris, G. Queisser, and G. Wittum. Simultaneous modelling of the extracellular and innercellular potential and the membrane voltage. Conference Abstract: Bernstein Conference on Computational Neuroscience, 2009.

[90] G. Pucihar, T. Kotnik, B. Valic, and D. Miklavcic. Numerical determination of transmembrane voltage induced on irregularly shaped cells. Annals of biomedical engineering, 34(4):642-652, April 2006.

[91] J. S. Rao. Finite Element Methods History of Rotating Machinery Dynamics. volume 20 of History of Mechanism and Machine Science, chapter 13, pages 141183. Springer Netherlands, Dordrecht, 2011.

[92] Philippe G. Ciarlet. The Finite Element Method for Elliptic Problems. Classics in applied mathematics, 40. SIAM: Society for Industrial and Applied Mathematics, 2nd edition, April 2002.

[93] Peter J. Olver and Cheri Shakiban. Applied Linear Algebra. Prentice Hall, 1 edition, January 2005.

[94] Peter J. Olver. Finite Element Methods. Lecture notes, University of Minnesota, 2012.

[95] Barbara I. Wohlmuth. Discretization Methods and Iterative Solvers Based on Domain Decomposition. Lecture Notes in Computational Science and Engineering. Springer, 1 edition, March 2001.

[96] Andrea Toselli and Olof Widlund. Domain Decomposition Methods - Algorithms and Theory, volume 34 of Springer Series in Computational Mathematics. Springer-Verlag, Berlin/Heidelberg, 2005.

[97] B. P. Lamichhane and B. I. Wohlmuth. Mortar finite elements for interface problems. Computing, 72:333-348, May 2004.

[98] E. J. Vigmond, F. Aguel, and N. A. Trayanova. Computational techniques for solving the bidomain equations in three dimensions. Biomedical Engineering, IEEE Transactions on, 49(11):1260-1269, November 2002.

[99] Pras Pathmanathan, Miguel O. Bernabeu, Rafel Bordas, Jonathan Cooper, Alan Garny, Joe M. Pitt-Francis, Jonathan P. Whiteley, and David J. Gavaghan. A numerical guide to the solution of the bi-domain equations of cardiac electrophysiology. Progress in biophysics and molecular biology, 102(2-3):136-155, June 2010 . 
[100] Marc Ethier and Yves Bourgault. Semi-Implicit Time-Discretization Schemes for the Bidomain Model. SIAM Journal on Numerical Analysis, 46(5):2443-2468, January 2008.

[101] Magnus R. Hestenes and Eduard Stiefel. Methods of Conjugate Gradients for Solving Linear Systems. Journal of Research of the National Bureau of Standards, 49(6):409-436, December 1952.

[102] Y. Saad and M. H. Schultz. GMRES: A generalized minimal residual algorithm for solving nonsymmetric linear systems. SIAM J. Sci. Stat. Comput., 7:856-869, 1986.

[103] Satish Balay, Jed Brown, Kris Buschelman, Victor Eijkhout, William D. Gropp, Dinesh Kaushik, Matthew G. Knepley, Lois C. McInnes, Barry F. Smith, and Hong Zhang. PETSc users manual. Technical Report ANL-95/11 - Revision 3.3, Argonne National Laboratory, 2012.

[104] William H. Press, Brian P. Flannery, Saul A. Teukolsky, and William T. Vetterling. Numerical Recipes in C: The Art of Scientific Computing, Second Edition. Cambridge University Press, 2 edition, October 1992.

[105] Lloyd N. Trefethen. Finite Difference and Spectral Methods for Ordinary and Partial Differential Equations. Department of Computer Science and Center for Applied Mathematics, Cornell University, 1996.

[106] Issai Schur. Neue Begründung der Theorie der Gruppencharaktere. Sitzungsberichte der Königlich Preußischen Akademie der Wissenschaften zu Berlin, 1905.

[107] James M. Bower and David Beeman. The book of GENESIS (2nd ed.): exploring realistic neural models with the GEneral NEural SImulation System. SpringerVerlag New York, Inc., New York, NY, USA, 1998.

[108] L. J. Borg-Graham. Additional efficient computation of branched nerve equations: adaptive time step and ideal voltage clamp. Journal of computational neuroscience, 8(3):209-226, 2000.

[109] Stefan Lang, Vincent J. Dercksen, Bert Sakmann, and Marcel Oberlaender. Simulation of signal flow in 3D reconstructions of an anatomically realistic neural network in rat vibrissal cortex. Neural networks : the official journal of the International Neural Network Society, 24(9):998-1011, November 2011.

[110] M. Hines. Efficient computation of branched nerve equations. International journal of bio-medical computing, 15(1):69-76, 1984.

[111] Michael Hines and Nicholas T. Carnevale. Computer modeling methods for neurons, chapter Computer modeling methods for neurons, pages 226-230. MIT Press, Cambridge, MA, USA, 1995.

[112] Erik De Schutter and David Beeman. Speeding Up GENESIS Simulations. Springer-Verlag New York, Inc., New York, NY, USA, 1998. 
[113] Antonius Otto. Methods of Numerical Simulation in Fluids and Plasmas. Lecture notes, University of Alaska, 2011.

[114] John C. Strikwerda. Finite difference schemes and partial differential equations. Society for Industrial and Applied Mathematics, 2004.

[115] Nicholas T. Carnevale and Michael L. Hines. The NEURON Book. Cambridge University Press, February 2006.

[116] Thomas H. Cormen, Charles E. Leiserson, Ronald L. Rivest, and Clifford Stein. Introduction to Algorithms. McGraw-Hill Science / Engineering / Math, 2nd edition, December 2003.

[117] Joe Pitt-Francis, Pras Pathmanathan, Miguel O. Bernabeu, Rafel Bordas, Jonathan Cooper, Alexander G. Fletcher, Gary R. Mirams, Philip Murray, James M. Osborne, and Alex Walter. Chaste: A test-driven approach to software development for biological modelling. Computer Physics Communications, 180(12):2452-2471, December 2009.

[118] COMSOL AB. The COMSOL Multiphysics Installation and Operations User's Guide, May 2012.

[119] ANSYS, Inc. ANSYS, Inc. Theory Reference, November 2004.

[120] F. Hecht. Freefem ++ Manual. Université Pierre et Marie Curie, third edition, May 2012.

[121] Anders Logg, Kent-Andre Mardal, and Garth Wells, editors. Automated Solution of Differential Equations by the Finite Element Method, volume 84. Springer Berlin Heidelberg, Berlin, Heidelberg, 2012.

[122] Edward J. Vigmond, Matt Hughes, G. Plank, and Joshua J. Leon. Computational tools for modeling electrical activity in cardiac tissue. Journal of electrocardiology, 36 Suppl:69-74, 2003.

[123] R. C. P. Kerckhoffs, S. N. Healy, T. P. Usyk, and A. D. McCulloch. Computational Methods for Cardiac Electromechanics. Proceedings of the IEEE, 94(4):769-783, April 2006.

[124] Alain Abran, Pierre Bourque, Robert Dupuis, James W. Moore, and Leonard L. Tripp. Guide to the Software Engineering Body of Knowledge - SWEBOK. IEEE Press, Piscataway, NJ, USA, 2004 version edition, 2004.

[125] Roger Pressman. Software Engineering: A Practitioner's Approach. McGrawHill., 5th edition, 2001.

[126] Joe Pitt-Francis, Miguel O. Bernabeu, Jonathan Cooper, Alan Garny, Lee Momtahan, James Osborne, Pras Pathmanathan, Blanca Rodriguez, Jonathan P. Whiteley, and David J. Gavaghan. Chaste: using agile programming techniques to develop computational biology software. Philosophical transactions. Series A, 
Mathematical, physical, and engineering sciences, 366(1878):3111-3136, September 2008 .

[127] G. van Rossum. Python Reference Manual. Technical report, Centrum voor Wiskunde en Informatica (CWI), May 1995.

[128] David N. Jansen, Holger Hermanns, and Joost-Pieter Katoen. UML 2003 - The Unified Modeling Language. 2003.

[129] Henry C. Tuckwell. Introduction to Theoretical Neurobiology: Volume 2, Nonlinear and Stochastic Theories, volume 2. Cambridge University Press, April 1988.

[130] S. Cohen and C. Hindmarsh. CVODE, A Stiff/nonstiff Ode Solver In C. Computers in Physics, 10(2):138-143, March 1996.

[131] Miguel O. Bernabeu, Rafel Bordas, Pras Pathmanathan, Joe Pitt-Francis, Jonathan Cooper, Alan Garny, David J. Gavaghan, Blanca Rodriguez, James A. Southern, and Jonathan P. Whiteley. Chaste: incorporating a novel multi-scale spatial and temporal algorithm into a large-scale open source library. Philosophical Transactions of the Royal Society A: Mathematical, Physical and Engineering Sciences, 367(1895):1907-1930, May 2009.

[132] S. Vassanelli and P. Fromherz. Transistor records of excitable neurons from rat brain. Applied Physics A: Materials Science \& Processing, V66(4):459-463, April 1998.

[133] Andrea Marcantoni, Valentina Carabelli, Valentina Comunanza, Daniela Gavello, Jonathan R. Ruiz, and Emilio Carbone. Microelectrode array (MEA) analysis of ion channels controlling spontaneous Chromaffin cells electrical activity. In 6th International Meeting on SubstrateIntegrated Micro Electrode Arrays, pages 70-72, 2008.

[134] E. Meyer, C. O. Müller, and P. Fromherz. Cable properties of dendrites in hippocampal neurons of the rat mapped by a voltage-sensitive dye. The European journal of neuroscience, 9(4):778-785, April 1997.

[135] Joseph D. Bronzino. Biomedical Engineering Fundamentals. CRC Press, 3 edition, April 2006.

[136] James B. Ranck. Specific impedance of rabbit cerebral cortex. Experimental Neurology, 7(2):144-152, February 1963.

[137] Z. F. Mainen and T. J. Sejnowski. Influence of dendritic structure on firing pattern in model neocortical neurons. Nature, 382(6589):363-366, July 1996.

[138] D. S. Tuch, V. J. Wedeen, A. M. Dale, J. S. George, and J. W. Belliveau. Conductivity tensor mapping of the human brain using diffusion tensor MRI. Proceedings of the National Academy of Sciences of the United States of America, 98(20):11697-11701, September 2001. 
[139] J. G. Jefferys. Nonsynaptic modulation of neuronal activity in the brain: electric currents and extracellular ions. Physiological reviews, 75(4):689-723, October 1995 .

[140] R. Plonsey and R. C. Barr. Electric field stimulation of excitable tissue. IEEE engineering in medicine and biology magazine : the quarterly magazine of the Engineering in Medicine 8 Biology Society, 17(5):130-137, 1998.

[141] D. Thurbon, A. Field, and S. Redman. Electrotonic profiles of interneurons in stratum pyramidale of the CA1 region of rat hippocampus. Journal of neurophysiology, 71(5):1948-1958, May 1994.

[142] Nelson Spruston, David B. Jaffe, and Daniel Johnston. Dendritic attenuation of synaptic potentials and currents: the role of passive membrane properties. Trends Neurosci, 17(4):161-166, January 1994.

[143] Leif Oltedal, Margaret Lin, and Espen Hartveit. Passive membrane properties and electrotonic signal processing in retinal rod bipolar cells. The Journal of physiology, 587(Pt 4):829-849, February 2009.

[144] G. Major, A. U. Larkman, P. Jonas, B. Sakmann, and J. J. Jack. Detailed passive cable models of whole-cell recorded CA3 pyramidal neurons in rat hippocampal slices. The Journal of neuroscience : the official journal of the Society for Neuroscience, 14(8):4613-4638, August 1994.

[145] Andrew J. Trevelyan and Julian Jack. Detailed passive cable models of layer $2 / 3$ pyramidal cells in rat visual cortex at different temperatures. The Journal of physiology, 539(Pt 2):623-636, March 2002.

[146] M. Rapp, I. Segev, and Y. Yarom. Physiology, morphology and detailed passive models of guinea-pig cerebellar Purkinje cells. The Journal of physiology, 474(1):101-118, January 1994.

[147] Bertil Hille. Ion Channels of Excitable Membranes. Sinauer Associates Inc 200107, 3rd edition edition, July 2001.

[148] HenryC Tuckwell. Voltage clamp calculations for myelinated and demyelinated axons. European Biophysics Journal, 22(1):71-77, April 1993.

[149] Sabyasachi Sircar. Principles of Medical Physiology. Thieme, 1 edition, January 2008.

[150] L. G. Nowak and J. Bullier. Axons, but not cell bodies, are activated by electrical stimulation in cortical gray matter. I. Evidence from chronaxie measurements. Experimental brain research. Experimentelle Hirnforschung. Expérimentation cérébrale, 118(4):477-488, February 1998.

[151] Maarten H. P. Kole, Susanne U. Ilschner, Bjorn M. Kampa, Stephen R. Williams, Peter C. Ruben, and Greg J. Stuart. Action potential generation requires a high 
sodium channel density in the axon initial segment. Nat Neurosci, 11(2):178-186, February 2008.

[152] G. Baranauskas, A. Mukovskiy, F. Wolf, and M. Volgushev. The determinants of the onset dynamics of action potentials in a computational model. Neuroscience, 167(4):1070-1090, June 2010.

[153] Yuguo Yu, Yousheng Shu, and David A. McCormick. Cortical Action Potential Backpropagation Explains Spike Threshold Variability and Rapid-Onset Kinetics. The Journal of Neuroscience, 28(29):7260-7272, July 2008.

[154] Stefan Hallermann, Christiaan P. J. de Kock, Greg J. Stuart, and Maarten H. P. Kole. State and location dependence of action potential metabolic cost in cortical pyramidal neurons. Nat Neurosci, 15(7):1007-1014, July 2012.

[155] Russell K. Hobbie and Bradley J. Roth. Intermediate Physics for Medicine and Biology. Springer, 4th edition, March 2007.

[156] Rivka S. Gold. The Axon Guide for Electrophysiology 63 Biophysics Laboratory Techniques. 2007.

[157] Paolo M. Rossini and Simone Rossi. Transcranial magnetic stimulation: diagnostic, therapeutic, and research potential. Neurology, 68(7):484-488, February 2007.

[158] Markus Kosel, Caroline Frick, Sarah H. Lisanby, Hans-Ulrich U. Fisch, and Thomas E. Schlaepfer. Magnetic seizure therapy improves mood in refractory major depression. Neuropsychopharmacology, 28(11):2045-2048, November 2003.

[159] Jennifer L. Boyer, Stephenie Harrison, and Tony Ro. Unconscious processing of orientation and color without primary visual cortex. Proceedings of the $\mathrm{Na}$ tional Academy of Sciences of the United States of America, 102(46):16875-16879, November 2005.

[160] Joseph T. Devlin and Kate E. Watkins. Stimulating language: insights from TMS. Brain, 130(3):610-622, March 2007.

[161] Liane Young, Joan A. Camprodon, Marc Hauser, Alvaro Pascual-Leone, and Rebecca Saxe. Disruption of the right temporoparietal junction with transcranial magnetic stimulation reduces the role of beliefs in moral judgments. Proceedings of the National Academy of Sciences, 107(15):6753-6758, April 2010.

[162] Ying-Zu Huang, Mark J. Edwards, Elisabeth Rounis, Kailash P. Bhatia, and John C. Rothwell. Theta Burst Stimulation of the Human Motor Cortex. 45(2):201-206, January 2005.

[163] Abraham Zangen, Yiftach Roth, Bernhard Voller, and Mark Hallett. Transcranial magnetic stimulation of deep brain regions: evidence for efficacy of the H-coil. Clinical neurophysiology, 116(4):775-779, April 2005. 
[164] J. P. Brasil-Neto, L. G. Cohen, M. Panizza, J. Nilsson, B. J. Roth, and M. Hallett. Optimal focal transcranial magnetic activation of the human motor cortex: effects of coil orientation, shape of the induced current pulse, and stimulus intensity. Journal of clinical neurophysiology, 9(1):132-136, January 1992.

[165] K. R. Mills, S. J. Boniface, and M. Schubert. Magnetic brain stimulation with a double coil: the importance of coil orientation. Electroencephalography and clinical neurophysiology, 85(1):17-21, February 1992.

[166] A. Pascual-Leone, L. G. Cohen, J. P. Brasil-Neto, and M. Hallett. Non-invasive differentiation of motor cortical representation of hand muscles by mapping of optimal current directions. Electroencephalography and clinical neurophysiology, 93(1):42-48, February 1994.

[167] P. Dubach. Significance of coil orientation for motor evoked potentials from nasalis muscle elicited by transcranial magnetic stimulation. Clinical Neurophysiology, 115(4):862-870, April 2004.

[168] T. Kammer, S. Beck, A. Thielscher, U. Laubis-Herrmann, and H. Topka. Motor thresholds in humans: a transcranial magnetic stimulation study comparing different pulse waveforms, current directions and stimulator types. Clinical neurophysiology, 112(2):250-258, February 2001.

[169] Tobias Tings, Nicolas Lang, Frithjof Tergau, Walter Paulus, and Martin Sommer. Orientation-specific fast rTMS maximizes corticospinal inhibition and facilitation. Experimental brain research. Experimentelle Hirnforschung. Expérimentation cérébrale, 164(3):323-333, July 2005.

[170] Assaf Rotem, Andres Agudelo-Toro, David Rakhmilevitch, Andreas Neef, and Elisha Moses. Efficient, orientation free magnetic stimulation of neurons with oscillating Field TMS. To be submitted, 2012.

[171] Milton Abramowitz and Irene A. Stegun. Handbook of Mathematical Functions: with Formulas, Graphs, and Mathematical Tables. Dover books on mathematics. Dover, New York, 1st edition, June 1964.

[172] E. Catmull and R. Rom. A class of local interpolating splines. Computer aided geometric design. Academic Press., pages 317-326, 1974.

[173] F. S. Salinas, J. L. Lancaster, and P. T. Fox. Detailed 3D models of the induced electric field of transcranial magnetic stimulation coils. Physics in medicine and biology, 52(10):2879-2892, May 2007.

[174] Gytis Baranauskas and Marco Martina. Sodium Currents Activate without a Hodgkin and Huxley-Type Delay in Central Mammalian Neurons. The Journal of Neuroscience, 26(2):671-684, January 2006.

[175] M. Migliore, L. Messineo, M. Cardaci, and G. F. Ayala. Quantitative modeling of perception and production of time intervals. J Neurophysiol, 86(6):2754-2760, December 2001. 


\section{List of Figures}

1.1 The process of Serial Block-Face Scanning Electron Microscopy [1] for reconstruction of a block of Mouse brain [2]. (Images (C) of TED Conferences LLC free for non commercial use under Creative Commons license "Attribution - NonCommercial - NonDerivative") . . . . . . . . . . .

1.2 Alternative representations in the cable equation (middle, right) for a spherical cell body (left). The electric field (E) is perpendicular to the axis of the body on the right. The problems of this representation are presented in Chapter 7. . . . . . . . . . . . . . . . . .

1.3 Modeling capabilities (top) and simulation results (A-F) with CHASTEMembrane. Bottom figures are described in Figure 8 of Chapter 7. . . .

2.1 Illustration of endogenous current sources in the volume conductor. In the volume conductor (white) the quasi-static approximation applies and it is assumed that no electric field sources from free charges exist. Endogenous current sources (gray) do not belong to the volume conductor but act as boundary conditions and can introduce currents, and hence electric fields, to it. . . . . . . . . . . . . . . . .

2.2 Schematic of the cell and volume regions. Volume $B$ can either intercept or not the membrane. . . . . . . . . . . . . . . . . . . . .

2.3 Close look to the volume intersecting the membrane. The volume is partitioned in an extracellular $B_{e}$ and an intracellular part $B_{i}$. . . . . . 32

2.4 A small imaginary cylinder sitting on top of a piece of membrane. . . . 35

2.5 A view of the volumetric representation of the cable . . . . . . . . . 37

2.6 Schematic of the solution domain (SD) and example boundary conditions

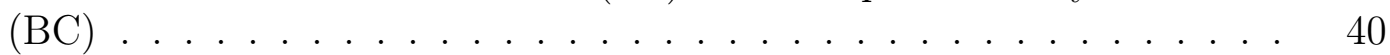

2.7 Projection of the electric field along a finite cable. . . . . . . . . . 43

3.1 Analytic solution of the infinite parallel membranes problem. . . . . . . 51

3.2 Illustration and analytic solution of the response of a short neuronal cable to a homogeneous extracellular pulse. . . . . . . . . . . . . . .

4.1 The finite element algorithm for a cell (light red) and extracellular space (blue) geometry. A special condition in this problem is that the membrane will represent an additional mesh. The right most figure represents the non zero elements of the coefficient matrix. . . . . . . . . . . . . . 
5.1 Examples of the circular and spherical cells of changing diameter and $h$ parameter. . . . . . . . . . . . . . . . . .

5.2 Experimental results finding the critical time step for a circular and spherical cell. . . . . . . . . . . . . . . . 83

5.3 Stability restriction for the space independent recurrence relation. . . . 86

5.4 Section of a regular 2D triangulation . . . . . . . . . . . . 86

5.5 Stability restriction for the space dependent recurrence relation. . . . . 90

5.6 Stability restriction on the space dependent von Neumann analysis . . . 93

5.7 Experimental stability criterion results for a cubic cell. . . . . . . . . . 95

5.8 Distribution of tetrahedra volumes for a spherical cell mesh . . . . . . . 95

6.1 The software architecture of CHASTE-Membrane. The tools developed during this thesis are presented in light-red. . . . . . . . . . . . . . . . 102

6.2 A typical simulation pipeline. Flow is described in Chapter B. . . . . . 104

8.1 Example of a mesh in which the cell (black circle) was partitioned through. Colors represent different partitions of the mesh. Alternatively, the cell could have been assigned to a color single region. What would be the best strategy for parallelization? Is communication within the parallel nodes significantly increased when they share a cell? . . . . . . . . . .

A.1 Membrane resistance vs. length constant for three different values of intracellular resistivity $\left(50,100,200 \mathrm{Ohm}^{*} \mathrm{~cm}\right)$ and two different diameters $(1$ and $10 \mu \mathrm{m})$. At about $150 \mathrm{Ohm}^{*} \mathrm{~cm}$ the length time constant of unmyelinated axons should be lower than $1 \mathrm{~mm}$ for realistic membrane resitances. . . . . . . . . . . . . . . . . .

A.1 Rotating cloverleaf coil in action. The induced electric field is displayed on top while the magnitude over time is displayed in the bottom.

A.2 Difference between different solutions with increasingly smaller length differential. . . . . . . . . . . . . . . . .

A.3 Spline reconstruction of the coil. (A) Spline (green) identifies the borders of the coil. (B-C) the final reconstructed spline of the coil. . . . . . . .

A.4 (A) complex vs. simple coil. (B-C) The field produced by the coils in the plane. (D-E) The field produced by the coils in Z. . . . . . . . . . .

A.5 Comparison of several coil shapes (A). (B) The horizontal and vertical components of the currents. (C) Time evolution of the magnitude of the induced field. (D-E) Field magnitudes at peak stimulus for the dashed lines in (A).

A.6 The cloverleaf coil alleviates the orientation dependence of magnetic stimulation in a passive axon. . . . . . . . . . . . . .

A.7 The cloverleaf coil alleviates the orientation dependence of magnetic stimulation in an active axon. . . . . . . . . . . . . . . . .

A.1 General inheritance dependence of CHASTE-Membrane objects (light red) and CHASTE objects (white). 
A.2 Object inheritance dependence for the matrix assembly and solving objects of CHASTE-Membrane (light red) and CHASTE (white). . . . . .

A.3 Object sequential communication of CHASTE-Membrane objects (light red) and CHASTE objects (white) during the main program execution.

A.4 Object sequential communication of CHASTE-Membrane objects (light red) and CHASTE objects (white) during the main solution loop. . . .

A.5 Object sequential communication of CHASTE-Membrane objects (light red) and CHASTE objects (white) during the matrix assembly loop. . . 


\section{Curriculum Vitae}

Andres Agudelo-Toro, +49-(0)551 3851-484. Deutsches Primatenzentrum GmbH. Kellnerweg 4, 37077 Göttingen, Deutschland.

\section{Education}

- Ph.D. in Theoretical and Computational Neuroscience, Max Planck Institute for Dynamics and SelfOrganization, Göttingen, Germany (2008-2012), Dissertation: "Numerical Simulations on the Biophysical Foundations of the Neuronal Extracellular Space". Supervised by Dr. Andreas Neef.

- M.Sc. in Applied Mathematics, EAFIT University, Colombia (2006-2008), graduated with honors. Thesis: "Finite State Automaton and Trajectory Optimization for the Hybrid Control of a Small Autonomous Helicopter". Overall GPA 4.35/5.00.

- B.S. Deg. in Computer Science, EAFIT University, Colombia (1999-2004), graduated with honors. Overall GPA 4.30/5.00 (highest score of the class 2004/1).

\section{Work experience}

- 2013- German Primate Center, Neurobiology Lab. Development of a real time neuronal decoding interface for control of a prosthetic arm. System integration of a robotic hand and arm for real time control.

- 2008-2013 MPI Dynamics and Self-organization, Theoretical Neurophysics group. Studied electromagnetic fields in biological tissue with applications to the stimulation and extracellular recording of neurons. Developed a numerical method for the full 3D solution of the quasi-static approximation of Maxwell's equations in neuronal tissue. Modeled the effects of magnetic and electric stimulation in neurons on $1 \mathrm{D}$ cell cultures.

- 2005-2007 EAFIT University, Control Systems Laboratory. Graduate research assistant. Developed a finite state automaton and trajectory optimizer for the controller of a robot helicopter. Analyzed techniques for linear control systems transitions based on the Catmull-Rom Spline. Translated a real-time control system developed in Matlab/Simulink to $\mathrm{C}$ code for real-time simulation and deployment in an embedded PC-104 computer.

- 2003-2004 Rutgers University, CAIP Center. Undergraduate visiting researcher. Assembled a computer vision system for pattern recognition with reconstructed camera parameters. Helped developing a multimodal tele-collaboration system for a DARPA demonstration.

- 2004-2007 EAFIT University, Virtual Reality Laboratory. Undergraduate researcher. Developed an object oriented memory model (graph based) to represent the environment in augmented and virtual reality applications. Built two electromagnetism educational modules for a distance learning platform.

\section{Key publications}

- A. Agudelo-Toro, A. Neef. Computationally efficient simulation of electrical activity at cell membranes interacting with self-generated and externally imposed electric fields. IOP Journal of Neural Engineering, $10026019,2013$.

- A. Agudelo-Toro, C. M. Velez. Transition Management for the Smooth Flight of a Small Autonomous Helicopter. Journal of Intelligent and Robotic Systems. Vol. 58, No. 1, pp 69-94, 2010.

- C. D. Correa, A. Agudelo-Toro, A. M. Krebs, I. Marsic, J. Hou, A. Morde, and S. K. Ganapathy. The Parallel Worlds System for Collaboration among Virtual and Augmented Reality Users. IEEE and ACM International Symposium on Mixed and Augmented Reality. Arlington, VA, 2004. 